Minimally invasive approach to diseases of the breast and contemporary factors affecting breast cancer surgery

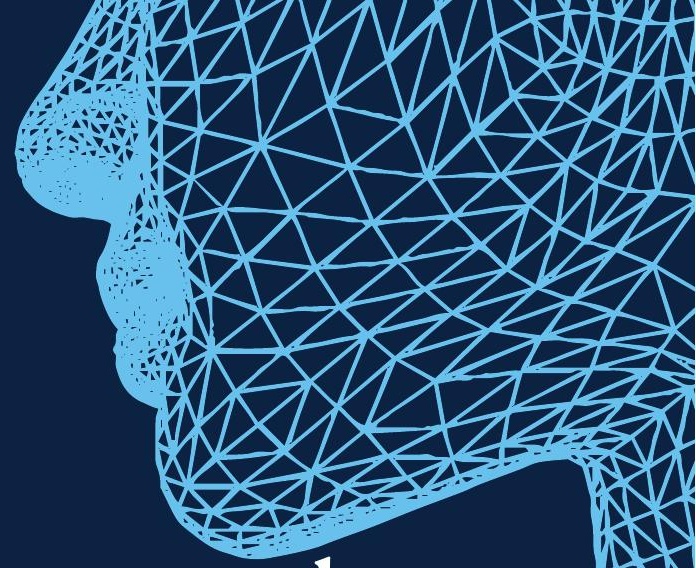




\section{Minimally invasive approach to diseases of the breast and contemporary factors affecting breast cancer surgery}

Mando Dyko Filipe 
ISBN: $\quad$ 978-94-6416-545-6

Cover design: $\quad$ Publiss | www.publiss.nl

Lay-out \& Print: Ridderprint | www.ridderprint.nl

(C) Copyright 2020: Mando Dyko Filipe, Utrecht, The Netherlands

All rights reserved. No part of this publication may be reproduced, stored in a retrieval system, or transmitted in any form or by any means, electronic, mechanical, by photocopying, recording, or otherwise, without the prior written permission of the author. 


\section{Minimally invasive approach to diseases of the breast and contemporary factors affecting breast cancer surgery}

Minimaal invasieve benadering van borstaandoeningen en hedendaagse factoren die van invloed zijn op borstkankerchirurgie (met een samenvatting in het Nederlands)

Proefschrift

ter verkrijging van de graad van doctor aan de

Universiteit Utrecht

op gezag van de

rector magnificus, prof.dr. H.R.B.M. Kummeling,

ingevolge het besluit van het college voor promoties

in het openbaar te verdedigen op donderdag 17 juni 2021 des middags te 2.15 uur

door

Mando Dyko Filipe

geboren op 24 december 1989

te Utrecht 


\section{Promotoren:}

Prof. dr. M.R. Vriens

Prof. dr. P.J. van Diest

\section{Copromotor:}

Dr. A.J. Witkamp

Dit proefschrift werd (mede) mogelijk gemaakt met financiële steun van TTW-KWF,

Van Herk Ventures, Leander Healthcare B.V en SeederDeBoer . 



\section{Contents}

Chapter 1. General outline 9

\section{Part I: Intraductal diagnosis of breast lesions.}

Chapter 2. Network meta-analysis for the diagnostic approach to pathologic

nipple discharge

Chapter 3. Interventional ductoscopy as an alternative for major duct excision or microdochectomy in gomen suffering pathologic nipple discharge: a single-center experience.

Chapter 4. Detection of breast cancer precursor lesions by autofluorescence ductoscopy.

Chapter 5. Feasibility of narrow band imaging ductoscopy, intraductal biopsy and intraductal laser ablation in patients with pathological nipple discharge and patients with a high-risk for the development of breast cancer: a study protocol.

\section{Part II: Minimally invasive treatment of diseases of the breast.}

Chapter 6. Cost-effectiveness analysis, systematic review and meta-analysis of ductoscopy, duct excision surgery and MRI for the diagnosis and treatment of patients with pathological nipple discharge.

Chapter 7. Patient reported outcomes of patients undergoing ductoscopy for pathological nipple discharge without radiological suspicion of malignancy.

Chapter 8. Ductoscopy for pathological nipple discharge (7 years later).

Chapter 9. Systematic review and meta-analysis comparing short-term effects of robot assisted nipple sparring mastectomy and conventional nipple sparring mastectomy. 


\section{Part III: Influence of socioeconomic status and COVID-19 on breast cancer treatment.}

Chapter 10. The association of socioeconomic status on treatment strategy in patients with stage I and II breast cancer in The Netherlands.

Chapter 11. Socioeconomic status significantly contributes to the likelihood of immediate postmastectomy breast reconstruction in the Netherlands: a nationwide study.

Chapter 12. Effect of the COVID-19 pandemic on surgical breast cancer care in the Netherlands: a multicenter retrospective cohort study.

\section{Part IV: Summary and general discussion.}

Chapter 13. General discussions, future perspectives and conclusions. 257

$\begin{array}{lll}\text { Chapter 14. Summary } & 265\end{array}$

$\begin{array}{lll}\text { Chapter 15. Addenda } & 273\end{array}$

Summary in Dutch (Nederlandse vertaling) 274

$\begin{array}{ll}\text { Author affiliation } & 279\end{array}$

Review committee $\quad 281$

List of publications $\quad 282$

Acknowledgements (Dankwoord) 284

About the author 289 


$$
1
$$


Chapter 1

General outline 


\section{General Outline}

\section{Pathological nipple discharge}

Pathologic nipple discharge (PND) is defined as spontaneous unilateral bloody or serous discharge from a single orifice of the nipple. It is a common breast-related complaint for referral due to its association with breast cancer. ${ }^{1-4}$ However, when ultrasound and mammography are negative, $95 \%$ of the PND have a benign cause (duct ectasias and intraductal papillomas).,

\section{Diagnostic work-up of PND}

Mammography and breast ultrasound are the commonly used diagnostic tools for the detection of breast cancer. However, when PND is the only symptom, they often miss breast cancer. ${ }^{7}$ Conversely, magnetic resonance imaging (MRI) has a high true positive rate for the detection of breast cancer but at the same time shows a high number of false positive results in patients with PND. ${ }^{8,9}$

\section{Treatment strategies for PND}

Microdochectomy and major duct excision are currently the most common used methods to rule out malignancy and treat patients with PND without radiological signs of malignancy. ${ }^{5,8}$ These surgical procedures are performed under general anesthesia and are associated with scarring, which may result in breastfeeding difficulties in fertile women and loss of sensitivity in the nipple. ${ }^{10}$

\section{Ductoscopy}

Ductoscopy is a minimally invasive micro-endoscopic technique providing real-time visualization of the milk ducts of the breast. ${ }^{11,12}$ Ductoscopy was developed in the early 1990 s in Japan and is currently standard practice in China and Japan to determine the cause of PND. ${ }^{13}$ Ductoscopy is performed under local anesthesia at the outpatient clinic and is currently used as a diagnostic tool in the work-up of women suffering from PND. ${ }^{14-19}$

(Pre)malignant epithelial lesions show a different pattern under fluorescent light. This has been used for different endoscopic procedures, such as bronchoscopy and colonoscopy, in order to detect lesions that were otherwise not visible. ${ }^{20-22}$ Ductoscopy has shown to have a high specificity but a low sensitivity for the detection of breast cancer. ${ }^{23}$ Therefore, the addition of autofluorescence could increase diagnostic accuracy for (pre)cancerous lesions during ductoscopy. Additionally, a previous study has shown the feasibility of autofluorescence ductoscopy in the ex-vivo setting. ${ }^{24}$ However, no clinical studies have 
been published. Another promising enhanced imaging technique is narrow band imaging (NBI). This technique allows superior visualization of vascularization. ${ }^{25}$ and improved detection of precancerous lesions during gastrointestinal scopic procedures and cystoscopy. ${ }^{25-28}$ Since (pre)cancerous breast tumors also have increased vascularization, ${ }^{29-33}$ it is plausible that NBI could also improve the sensitivity for the detection of (pre) malignant lesions during ductoscopy.

Current biopsy tools (such as the endobasket) do not always succeed in removing lesions causing PND completely. ${ }^{12,34}$ Laser ablation techniques are widely used and have proven to be safe and feasible to evaporate (pre)malignant and non-malignant lesions. ${ }^{35,36}$ Therefore, intraductal laser ablation could help removing these remnants thereby reducing the need for duct excision surgery in patients with PND. Furthermore, safety and feasibility of intraductal laser ablation has previously been determined in the ex vivo setting. ${ }^{37}$

\section{Breast cancer}

Breast cancer is the most common form of cancer in women and the second most common cause of cancer related death in women worldwide. ${ }^{38}$ The annual incidence of breast cancer is approximately 17.000 in the Netherlands alone, claiming the lives of 3.000 women each year. ${ }^{39,40} \mathrm{Up}$ to $5-10 \%$ of all breast cancers cases have an hereditary component. Mutations in BRCA1 and BRCA2, the two most important breast cancer related genes, have a $60 \%-80 \%$ lifetime risk of breast cancer and account for $16 \%$ of familial cases of breast cancer. ${ }^{41,42}$

Most breast cancer patients are treated by breast conserving surgery followed by adjuvant therapy (breast conserving therapy, BCT) or mastectomy, depending on tumor characteristics. ${ }^{43,44}$

Bilateral prophylactic mastectomy is recommended for patients with an increased risk of developing breast cancer, and is therefore currently recommended in patients in patients with BRCA1 and BRCA2 mutations. ${ }^{45,46}$ Unfortunately, this is accompanied by complications, ${ }^{47}$ and serious cosmetic and psychological consequences. ${ }^{48}$ One of the most important challenges of nipple-sparing mastectomy is achieving adequate exposure to perform precise dissection in areas that are remote from the skin incision. Robotic nipple-sparing mastectomy (RNSM) is relatively new technique that allows for better visualization of tissue planes and exposed tissue that was challenging to reach with traditional nipple-sparing mastectomy techniques. ${ }^{49-51}$ Previous research has demonstrated feasibility and safety of RNSM, in addition to a steep learning curve. ${ }^{52,53}$ 


\section{Socioeconomic status and breast cancer treatment}

Socioeconomic status (SES) is a multi-layered system to stratify economic and social factors such as prestige and social status and has shown to influence the prevalence of a wide array of diseases. ${ }^{54} \mathrm{~A}$ low SES is associated with higher rates of diabetes, cardiovascular disease and many types of cancer in the western world. Furthermore, psychiatric disorders are more often seen in low SES patients. ${ }^{55-58}$

In the United States of America, breast cancer is detected at a later stage in patients with a low SES. This difference can be attributed to differences in access to healthcare and screening programs. Therefore, the therapeutic choice differs per SES due to tumor stage during diagnosis as well as potential financial barriers. ${ }^{59}$ However, a Dutch study shows that despite the lower incidence of breast cancer, the overall survival of breast cancer patients with a migration background is lower than their native Dutch counterparts. ${ }^{60}$ Yet, no differences in overall breast cancer survival are seen in different education levels in the Netherlands, which is strongly associated with SES. ${ }^{61}$ Lower breast cancer screening attendance in women with a low SES results in later stage at diagnosis, which may explain these differences. ${ }^{62,63}$

A systematic review showed that patients with breast cancer who, among other factors, had a higher SES were more likely to undergo BCT. ${ }^{64}$ There are different studies in the United States of America showing the effect of SES in choosing a surgical procedure. ${ }^{65,66}$ Furthermore, this Danish study showed that low SES stage I or II breast cancer patients tend to have more mastectomy procedures despite equal access to healthcare. There was no clear explanation for this disparity. ${ }^{67}$ However, this was not a population-sized cohort and treatment possibilities have changed since 1998.

\section{COVID-19 and breast cancer surgery}

Coronavirus disease 2019 (COVID-19) is a highly infectious disease caused by acute respiratory syndrome coronavirus 2 (SARS-CoV-2) and is responsible for the ongoing pandemic. The COVID-19 pandemic has posed a challenge to regular healthcare. In the Netherlands, similar policies were implemented regarding oncologic care ${ }^{68}$ If possible, elective surgical procedures were postponed, and the national screening programs for breast and colorectal cancer were temporarily halted since March 16, 2020 until half August $2020 .{ }^{69}$ This is unfavorable since with approximately $40 \%$ of new onset breast cancers are detected through the national screening program. ${ }^{39,40}$

Since surgery is the cornerstone of breast cancer treatment ${ }^{43,44}$ and there have been big reallocations of hospital resources due to the increased demand of COVID-19 care, $^{70,71}$ the impact of COVID-19 on breast cancer surgical care is expected to be substantial. 


\section{Thesis outline}

The aim of this thesis is to find new strategies for methods of minimally invasive approaches to diseases of the breast and to determine which factors contribute to breast cancer care. To this regard, the current value and future influence of ductoscopy for diagnosis of intraductal lesions of the breast are explored in part I of this thesis. Part II of this thesis focusses on the minimally invasive treatment approach, i.e. ductoscopy and robot assisted mastectomy, for diseases of the breast. The influence of socioeconomic factors of patients and the ongoing COVID-19 pandemic on surgical breast cancer care are explored in part III. 


\section{Research questions}

The research questions addressed in this thesis can be summarized as followed:

\section{Part I. Intraductal diagnosis of breast lesions}

Chapter 2 What is the best diagnostic tool for the detection of breast cancer in patients with pathological nipple discharge?

Chapter 3 Is ductoscopy a safe alternative for surgical intervention in patients with pathological nipple discharge with negative conventional imaging?

Chapter 4 Is it possible to enhance the diagnostic and therapeutic performance of ductoscopy?

Chapter 5 What is necessary to further increase the diagnostic validity of ductoscopy?

\section{Part II: Minimally invasive treatment of diseases of the breast}

Chapter 6 Is ductoscopy a cost-effective diagnostic and therapeutic in patients with pathological nipple discharge?

Chapter 7 What is the impact of ductoscopy from the patient's perspective?

Chapter 8 What are the developments of ductoscopy in the last 7 years?

Chapter 9 Is it safe to perform robot-assisted mastectomies?

\section{Part III. Influence of socioeconomic status and COVID-19 on breast cancer treatment}

Chapter 10 What is the influence of socioeconomic status on treatment in patients with new onset stage I or II breast cancer?

Chapter 11 What is the influence of socioeconomic status on breast reconstruction after mastectomy due to stage I or II breast cancer?

Chapter 12 What is the impact of the coronavirus disease 2019 (COVID-19) on breast cancer treatment in the center of the Netherlands? 


\section{References:}

1. Dixon JM, Mansel RE. ABC of breast diseases. Symptoms assessment and guidelines for referral. BMJ. 1994;309(6956):722-726.

2. Seltzer MH. Breast complaints, biopsies, and cancer correlated with age in 10,000 consecutive new surgical referrals. Breast J. 2004;10(2):111-117. doi:21284 [pii]

3. Santen RJ, Mansel R. Benign breast disorders. N Engl J Med. 2005;353(3):275-285. doi:353/3/275 [pii]

4. King TA, Carter KM, Bolton JS, Fuhrman GM. A simple approach to nipple discharge. Am Surg. 2000;66(10):960-966.

5. Alcock C, Layer GT. Predicting occult malignancy in nipple discharge. ANZ J Surg. 2010;80(9):646-649. doi:10.1111/j.1445-2197.2010.05270.x [doi]

6. Albrecht C, Thele F, Grunwald S, et al. Nipple discharge: role of ductoscopy in comparison with standard diagnostic tests. Onkologie. 2013;36(1-2):12-16. doi:10.1159/000346639 [doi]

7. Bahl M, Baker JA, Greenup RA, Ghate S V. Diagnostic Value of Ultrasound in Female Patients With Nipple Discharge. AJRAmerican J Roentgenol. 2015;205(1):203-208. doi:10.2214/AJR.14.13354 [doi]

8. Sanders LM, Daigle M. The Rightful Role of MRI after Negative Conventional Imaging in the Management of Bloody Nipple Discharge. Breast J. 2016;22(2):209-212. doi:10.1111/tbj.12551 [doi]

9. van Gelder L, Bisschops RH, Menke-Pluymers MB, Westenend PJ, Plaisier PW. Magnetic resonance imaging in patients with unilateral bloody nipple discharge; useful when conventional diagnostics are negative? World J Surg. 2015;39(1):184-186. doi:10.1007/s00268-014-2701-1 [doi]

10. Sarakbi W Al, Worku D, Escobar PF, Mokbel K. Breast papillomas: current management with a focus on a new diagnostic and therapeutic modality. Int Semin Surg Oncol. 2006;3:1. doi:1477-7800-3-1 [pii]

11. Yang X, Li H, Gou J, et al. The role of breast ductoscopy in evaluation of nipple discharge: a chinese experience of 419 patients. Breast J. 2014;20(4):388-393. doi:10.1111/tbj.12275 [doi]

12. Waaijer L, van Diest PJ, Verkooijen HM, et al. Interventional ductoscopy in patients with pathological nipple discharge. Br J Surg. 2015;102(13):1639-1648. doi:10.1002/bjs.9950 [doi]

13. Okazaki A, Okazaki M, Asaishi K, et al. Fiberoptic ductoscopy of the breast: a new diagnostic procedure for nipple discharge. Jpn J Clin Oncol. 1991;21(3):188-193.

14. Yamamoto D, Shoji T, Kawanishi H, et al. A utility of ductography and fiberoptic ductoscopy for patients with nipple discharge. Breast Cancer Res Treat. 2001;70(2):103-108.

15. Yamamoto D, Ueda S, Senzaki H, et al. New diagnostic approach to intracystic lesions of the breast by fiberoptic ductoscopy. Anticancer Res. 2001;21(6A):4113-4116.

16. Matsunaga T, Ohta D, Misaka T, et al. Mammary ductoscopy for diagnosis and treatment of intraductal lesions of the breast. Breast Cancer. 2001;8(3):213-221.

17. Grunwald S, Heyer H, Paepke S, et al. Diagnostic value of ductoscopy in the diagnosis of nipple discharge and intraductal proliferations in comparison to standard methods. Onkologie. 2007;30(5):243-248. doi: 100848 [pii]

18. Han Y, Li J, Han S, Jia S, Zhang Y, Zhang W. Diagnostic value of endoscopic appearance during ductoscopy in patients with pathological nipple discharge. BMC Cancer. 2017;17(1):300-303. doi:10.1186/s12885017-3288-3 [doi]

19. Kamali S, Bender O, Kamali GH, Aydin MT, Karatepe O, Yuney E. Diagnostic and therapeutic value of ductoscopy in nipple discharge and intraductal proliferations compared with standard methods. Breast Cancer. 2014;21(2):154-161. doi:10.1007/s12282-012-0377-7 [doi] 
20. Ogihara T, Watanabe H, Namihisa A, Kobayashi O, Miwa H, Sato N. Clinical experience using a real time autofluorescence endoscopy system in the gastrointestinal tract. Diagn Ther Endosc. 1999;5(2):119124. doi:10.1155/DTE.5.119 [doi]

21. Ikeda N, Honda H, Hayashi A, et al. Early detection of bronchial lesions using newly developed videoendoscopy-based autofluorescence bronchoscopy. Lung Cancer. 2006;52(1):21-27. doi:S0169-5002(06)00018-3 [pii]

22. van der Heijden EH, Hoefsloot W, van Hees HW, Schuurbiers OC. High definition bronchoscopy: a randomized exploratory study of diagnostic value compared to standard white light bronchoscopy and autofluorescence bronchoscopy. Respir Res. 2015;16:33-37. doi:10.1186/s12931-015-0193-7 [doi]

23. Waaijer L, Simons JM, Borel Rinkes IH, van Diest PJ, Verkooijen HM, Witkamp AJ. Systematic review and meta-analysis of the diagnostic accuracy of ductoscopy in patients with pathological nipple discharge. Br J Surg. 2016;103(6):632-643. doi:10.1002/bjs.10125 [doi]

24. Jacobs VR, Paepke S, Schaaf H, Weber BC, Kiechle-Bahat M. Autofluorescence ductoscopy: a new imaging technique for intraductal breast endoscopy. Clin Breast Cancer. 2007;7(8):619-623. doi:S1526-8209(11)70750-9 [pii]

25. Singh R, Mei SC, Sethi S. Advanced endoscopic imaging in Barrett's oesophagus: a review on current practice. World J Gastroenterol. 2011;17(38):4271-4276. doi:10.3748/wjg.v17.i38.4271 [doi]

26. Efthymiou M, Taylor AC, Kamm MA. Cancer surveillance strategies in ulcerative colitis: the need for modernization. Inflamm Bowel Dis. 2011;17(8):1800-1813. doi:10.1002/ibd.21540 [doi]

27. Tanaka S, Sano Y. Aim to unify the narrow band imaging (NBI) magnifying classification for colorectal tumors: current status in Japan from a summary of the consensus symposium in the 79th Annual Meeting of the Japan Gastroenterological Endoscopy Society. Dig Endosc. 2011;23 Suppl 1:131-139. doi:10.1111/ j.1443-1661.2011.01106.x [doi]

28. Jichlinski P, Lovisa B. High magnification cystoscopy in the primary diagnosis of bladder tumors. Curr Opin Urol. 2011;21(5):398-402. doi:10.1097/MOU.0b013e32834956ad [doi]

29. Ruiz A, Almenar S, Cerda M, Hidalgo JJ, Puchades A, Llombart-Bosch A. Ductal carcinoma in situ of the breast: a comparative analysis of histology, nuclear area, ploidy, and neovascularization provides differentiation between low- and high-grade tumors. Breast J. 2002;8(3):139-144. doi:tbj08303 [pii]

30. Santamaria G, Velasco M, Farrus B, Caparros FX, Fernandez PL. Dynamic contrast-enhanced MRI reveals the extent and the microvascular pattern of breast ductal carcinoma in situ. Breast J. 2013;19(4):402-410. doi: $10.1111 /$ tbj. 12135 [doi]

31. Gadre SA, Perkins GH, Sahin AA, Sneige N, Deavers MT, Middleton LP. Neovascularization in mucinous ductal carcinoma in situ suggests an alternative pathway for invasion. Histopathology. 2008;53(5):545553. doi:10.1111/j.1365-2559.2008.03152.x [doi]

32. Li L, Wang K, Sun X, et al. Parameters of dynamic contrast-enhanced MRI as imaging markers for angiogenesis and proliferation in human breast cancer. Med Sci Monit. 2015;21:376-382. doi:10.12659/ MSM.892534 [doi]

33. Fox SB, Generali DG, Harris AL. Breast tumour angiogenesis. Breast Cancer Res. 2007;9(6):216. doi:10.1186/bcr1796 [doi]

34. Filipe MD, Waaijer L, van der Pol CC, van Diest P, Witkamp AJ. Interventional ductoscopy as an alternative for major duct excision or microdochectomy in women suffering pathological nipple discharge: a single centre experience. Clin Breast Cancer. January 2020. doi:10.1016/j.clbc.2019.12.008

35. Netsch C, Engbert A, Bach T, Gross AJ. Long-term outcome following Thulium VapoEnucleation of the prostate. World J Urol. 2014;32(6):1551-1558. doi:10.1007/s00345-014-1260-2 [doi] 
36. Allam JP, Novak N. The pathophysiology of atopic eczema. Clin Exp Dermatol. 2006;31(1):89-93. doi:CED1980 [pii]

37. de Boorder T, Waaijer L, van Diest PJ, Witkamp AJ. Ex vivo feasibility study of endoscopic intraductal laser ablation of the breast. Lasers Surg Med. 2018;50(2):137-142. doi:10.1002/lsm.22745

38. Siegel RL, Miller KD, Jemal A. Cancer statistics, 2018. CA Cancer J Clin. 2018;68(1):7-30. doi:10.3322/ caac. 21442 [doi]

39. Vondeling GT, Menezes GL, Dvortsin EP, et al. Burden of early, advanced and metastatic breast cancer in The Netherlands. BMC Cancer. 2018;18(1):262-263. doi:10.1186/s12885-018-4158-3 [doi]

40. Koleva-Kolarova RG, Daszczuk AM, de Jonge C, et al. A modelling study to evaluate the costs and effects of lowering the starting age of population breast cancer screening. Maturitas. 2018;109:81-88. doi:S0378-5122(17)30543-1 [pii]

41. Ford D, Easton DF, Stratton M, et al. Genetic heterogeneity and penetrance analysis of the BRCA1 and BRCA2 genes in breast cancer families. The Breast Cancer Linkage Consortium. Am J Hum Genet. 1998;62(3):676-689. doi:S0002-9297(07)63848-8 [pii]

42. Tung N, Battelli C, Allen B, et al. Frequency of mutations in individuals with breast cancer referred for BRCA1 and BRCA2 testing using next-generation sequencing with a 25-gene panel. Cancer. 2015;121(1):25-33. doi:10.1002/cncr.29010 [doi]

43. Hartmann-Johnsen OJ, Karesen R, Schlichting E, Nygard JF. Survival is Better After Breast Conserving Therapy than Mastectomy for Early Stage Breast Cancer: A Registry-Based Follow-up Study of Norwegian Women Primary Operated Between 1998 and 2008. Ann Surg Oncol. 2015;22(12):38363845. doi:10.1245/s10434-015-4441-3 [doi]

44. van Maaren MC, de Munck L, de Bock GH, et al. 10 year survival after breast-conserving surgery plus radiotherapy compared with mastectomy in early breast cancer in the Netherlands: a population-based study. The LancetOncology. 2016;17(8):1158-1170. doi:S1470-2045(16)30067-5 [pii]

45. Casella D, Di Taranto G, Marcasciano M, et al. Nipple-sparing bilateral prophylactic mastectomy and immediate reconstruction with TiLoop $\left({ }^{\circ}\right)$ Bra mesh in BRCA1/2 mutation carriers: A prospective study of long-term and patient reported outcomes using the BREAST-Q. Breast. 2018;39:8-13. https://pubmed. ncbi.nlm.nih.gov/29455110.

46. Ludwig KK, Neuner J, Butler A, Geurts JL, Kong AL. Risk reduction and survival benefit of prophylactic surgery in BRCA mutation carriers, a systematic review. Am J Surg. 2016;212(4):660-669. doi:S0002-9610(16)30348-8 [pii]

47. Osman F, Saleh F, Jackson TD, Corrigan MA, Cil T. Increased postoperative complications in bilateral mastectomy patients compared to unilateral mastectomy: an analysis of the NSQIP database. Ann Surg Oncol. 2013;20(10):3212-3217. doi:10.1245/s10434-013-3116-1 [doi]

48. Eisen A, Rebbeck TR, Wood WC, Weber BL. Prophylactic surgery in women with a hereditary predisposition to breast and ovarian cancer. J Clin Oncol. 2000;18(9):1980-1995. doi:10.1200/ JCO.2000.18.9.1980 [doi]

49. Lai HW, Lin SL, Chen ST, et al. Robotic Nipple-sparing Mastectomy and Immediate Breast Reconstruction with Gel Implant. Plast Reconstr surgeryGlobal open. 2018;6(6):e1828. doi:10.1097/ GOX.0000000000001828 [doi]

50. Lai HW. Robotic Nipple-Sparing Mastectomy and Immediate Breast Reconstruction with Gel Implant. Ann Surg Oncol. 2019;26(1):53-54. doi:10.1245/s10434-018-6711-3 [doi]

51. Toesca A, Invento A, Massari G, et al. Update on the Feasibility and Progress on Robotic Breast Surgery. Ann Surg Oncol. 2019;26(10):3046-3051. doi:10.1245/s10434-019-07590-7 [doi] 
52. Houvenaeghel G, Bannier M, Rua S, et al. Breast cancer robotic nipple sparing mastectomy: evaluation of several surgical procedures and learning curve. World J Surg Oncol. 2019;17(1):27-019-1567-y. doi:10.1186/s12957-019-1567-y [doi]

53. Toesca A, Peradze N, Manconi A, et al. Robotic nipple-sparing mastectomy for the treatment of breast cancer: Feasibility and safety study. Breast. 2017;31:51-56. doi:S0960-9776(16)30191-6 [pii]

54. Adler NE, Boyce T, Chesney MA, et al. Socioeconomic status and health. The challenge of the gradient. Am Psychol. 1994;49(1):15-24.

55. Clegg LX, Reichman ME, Miller BA, et al. Impact of socioeconomic status on cancer incidence and stage at diagnosis: selected findings from the surveillance, epidemiology, and end results: National Longitudinal Mortality Study. Cancer Causes Control. 2009;20(4):417-435. doi:10.1007/s10552-008-9256-0 [doi]

56. Dohrenwend BP, Levav I, Shrout PE, et al. Socioeconomic status and psychiatric disorders: the causationselection issue. Science. 1992;255(5047):946-952.

57. Psaltopoulou T, Hatzis G, Papageorgiou N, Androulakis E, Briasoulis A, Tousoulis D. Socioeconomic status and risk factors for cardiovascular disease: Impact of dietary mediators. Hellenic J Cardiol. 2017;58(1):32-42. doi:S1109-9666(17)30040-4 [pii]

58. Saydah S, Lochner K. Socioeconomic status and risk of diabetes-related mortality in the U.S. Public Heal reports (Washington, DC 1974). 2010;125(3):377-388. doi:10.1177/003335491012500306 [doi]

59. Bradley CJ, Given CW, Roberts C. Race, socioeconomic status, and breast cancer treatment and survival. J Natl Cancer Inst. 2002;94(7):490-496.

60. Arnold M, Aarts MJ, Siesling S, Aa M, Visser O, Coebergh JW. Diverging breast and stomach cancer incidence and survival in migrants in The Netherlands, 1996-2009. Acta Oncol. 2013;52(6):1195-1201. doi:10.3109/0284186X.2012.742962 [doi]

61. Aarts MJ, Kamphuis CB, Louwman MJ, Coebergh JW, Mackenbach JP, van Lenthe FJ. Educational inequalities in cancer survival: a role for comorbidities and health behaviours? J Epidemiol Community Health. 2013;67(4):365-373. doi:10.1136/jech-2012-201404 [doi]

62. Aarts MJ, Hamelinck VC, Bastiaannet E, et al. Small but significant socioeconomic inequalities in axillary staging and treatment of breast cancer in the Netherlands. Br J Cancer. 2012;107(1):12-17. doi:10.1038/ bjc.2012.205 [doi]

63. Aarts MJ, Voogd AC, Duijm LE, Coebergh JW, Louwman WJ. Socioeconomic inequalities in attending the mass screening for breast cancer in the south of the Netherlands--associations with stage at diagnosis and survival. Breast Cancer Res Treat. 2011;128(2):517-525. doi:10.1007/s10549-011-1363-z [doi]

64. Gu J, Groot G, Boden C, Busch A, Holtslander L, Lim H. Review of Factors Influencing Women's Choice of Mastectomy Versus Breast Conserving Therapy in Early Stage Breast Cancer: A Systematic Review. Clin Breast Cancer. 2018;18(4):e539-e554. doi:S1526-8209(17)30691-2 [pii]

65. Bhat S, Orucevic A, Woody C, Heidel RE, Bell JL. Evolving Trends and Influencing Factors in Mastectomy Decisions. Am Surg. 2017;83(3):233-238.

66. Churilla TM, Egleston B, Bleicher R, Dong Y, Meyer J, Anderson P. Disparities in the Local Management of Breast Cancer in the US according to Health Insurance Status. Breast J. 2017;23(2):169-176. doi: $10.1111 /$ tbj. 12705 [doi]

67. Norredam M, Groenvold M, Petersen JH, Krasnik A. Effect of social class on tumour size at diagnosis and surgical treatment in Danish women with breast cancer. Soc Sci Med. 1998;47(11):1659-1663. doi:S0277953698002603 [pii]

68. NVVH. Handvat voor chirurgische ingrepen tijdens Corona-crisis. 2020. 
69. Dinmohamed AG, Visser O, Verhoeven RHA, et al. Fewer cancer diagnoses during the COVID-19 epidemic in the Netherlands. Lancet Oncol. April 2020. doi:10.1016/S1470-2045(20)30265-5

70. Truog RD, Mitchell C, Daley GQ. The Toughest Triage - Allocating Ventilators in a Pandemic. N Engl JMed. 2020;382(21):1973-1975. doi:10.1056/NEJMp2005689

71. Emanuel EJ, Persad G, Upshur R, et al. Fair Allocation of Scarce Medical Resources in the Time of Covid-19. N Engl J Med. 2020;382(21):2049-2055. doi:10.1056/NEJMsb2005114 


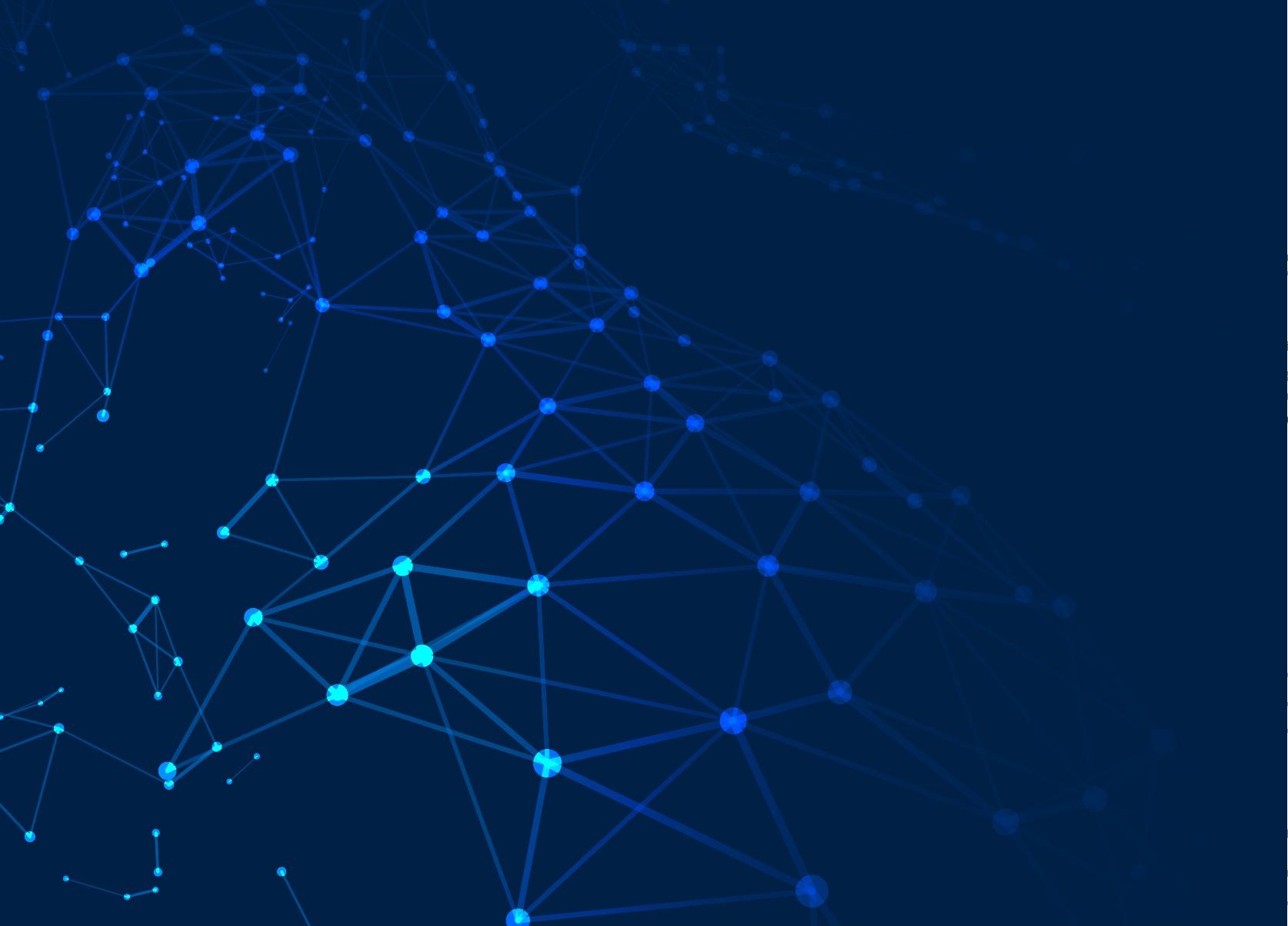





$$
2
$$




\section{Chapter 2}

Network meta-analysis for the diagnostic approach to pathologic nipple discharge

M.D. Filipe

S.I.S. Patuleia

V.M.T. de Jong

M.R. Vriens

P.J. van Diest

A.J. Witkamp 


\begin{abstract}
Introduction: Pathological nipple discharge (PND) is one of the most common breastrelated complaints for referral because of its supposed association with breast cancer. The aim of this network meta-analysis (NMA) was to compare the diagnostic efficacy of ultrasound, mammography, cytology, MRI and ductoscopy in patients with PND, as well as to determine the best diagnostic strategy to assess the risk of malignancy as cause for PND.
\end{abstract}

Materials and Methods: Cochrane Library, PubMed and EMBASE were searched to collect relevant literature from the inception of each of the diagnostic methods until January $27^{\text {th }}, 2020$. The search yielded 1472 original citations, of which 36 studies with 3764 patients were finally included for analysis. Direct and indirect comparisons were performed using an NMA approach to evaluate the combined odd ratios (OR) and determine the surface under the cumulative ranking curves (SUCRA) of the diagnostic value of different imaging methods for the detection of breast cancer in patients with PND. Additionally, a subgroup meta-analysis comparing ductoscopy to MRI when conventional imaging was negative was also performed.

Results: According to this NMA, sensitivity for detection of malignancy in patients with PND was highest for MRI (83\%), followed by ductoscopy (58\%), ultrasound (50\%), cytology $(38 \%)$ and mammography $(22 \%)$. Specificity was highest for mammography (93\%) followed by ductoscopy (92\%), cytology (90\%), MRI 76\% and ultrasound $(69 \%)$. Diagnostic accuracy was the highest for ductoscopy (88\%), followed by cytology (82\%), MRI (77\%), mammography (76\%) and ultrasound (65\%). Subgroup metaanalysis (comparing ductoscopy to MRI when ultrasound and mammography were negative) showed no significant difference in sensitivity but ductoscopy was statistically significantly better with regard to specificity and diagnostic accuracy.

Conclusion: The results from this NMA indicate that although ultrasound and mammography may remain low cost useful first choices for the detection of malignancy in patients with PND, ductoscopy outperforms most imaging techniques (especially MRI) and cytology. 


\section{Introduction}

Pathological nipple discharge (PND) is defined as unilateral, spontaneous and bloody or serous discharge, usually arising from a single duct orifice of the nipple. After pain and palpable lumps, PND is the third most common breast-related complaint ${ }^{1}$. PND is often associated with breast cancer and accounts for 3-5\% of surgical breast clinic referrals ${ }^{2-5}$. However, the most common causes of PND are benign (duct ectasias and intraductal papillomas) ${ }^{6,7}$.

Mammography and breast ultrasound are important tools for the detection of breast cancer. However, in the case of PND as the only complaint, they both have limited sensitivity ${ }^{8}$. Magnetic resonance imaging (MRI) has shown to be a sensitive tool for the detection of malignancy but specificity is low. Detection of small lesions and differentiating benign from malignant masses remains difficult with MRI ${ }^{9,10}$. Therefore, the value of MRI is limited in patients with PND and core needle biopsy or surgical excision is still necessary when MRI shows a suspicious lesion ${ }^{11,12}$. Cytology of the nipple discharge is also used to determine the risk of malignancy in patients with PND but its clinical relevance has been contested ${ }^{5,13,14}$.

Ductoscopy is a minimally invasive micro-endoscopic technique providing real-time visualization of the milk ducts of the breast. Ductoscopy is performed under local anaesthesia at the outpatient clinic and is currently used as a diagnostic tool in the workup of women suffering from PND ${ }^{15-22}$. Previous studies and a meta-analysis show that ductoscopy is a useful tool in finding intraductal lesions causing PND (benign and malignant) before or during duct excision ${ }^{23-25}$.

Since PND is regarded as a possible sign of breast cancer and standard radiological imaging often fails to reveal the cause, most women suffering persistent PND undergo surgical procedures, such as microdochectomy or major duct excision, to rule out malignancy 6,8,9. However, only 5-8\% of these patients with PND turn out to have malignancy $5,26,27$, meaning that $90-95 \%$ of these surgical procedures are performed for non-malignant causes. Therefore, it is important to assess the different diagnostic tools currently available and determine the usefulness of each tool in the different phases of the diagnostic process. To this end, we carried out a systematic review of the literature and performed a network meta-analysis (NMA) to compare the value of different diagnostic tools to detect malignancy in patients with PND. Additionally, we determined the optimal diagnostic strategy for patients with PND. 


\section{Materials and methods}

This systematic review and NMA was performed according to the guidelines of the requirements of the Preferred Reporting Items for Systematic Reviews and Meta-analysis (PRISMA) checklist for NMA (Supplemental Appendix 3) ${ }^{28}$. A systematic literature search was performed in the PubMed, Embase, and Cochrane Library databases. The search strategy was performed on all index tests (ultrasound, mammogram, MRI, cytology, and ductoscopy) and their synonyms. The full electronic search strategy can be found in Supplemental Appendix 1 in the online version. After removal of duplicates, two authors (M.F., S.P.) independently screened articles by title and abstract. The full articles were independently screened for eligibility based on predefined inclusion and exclusion criteria. Discordant judgments were discussed by the two authors until consensus was reached.

\section{Selection of studies}

Full texts were retrieved for studies that evaluated ultrasound, mammography, MRI, cytology and/or ductoscopy, reported original data and were written in English.

1. Participants: patients with PND as main breast complaint without history of breast cancer.

2. Intervention: ultrasound, mammography, MRI, cytology and/or ductoscopy.

3. Comparator: if patients were diagnosed with malignancy, they must have had definitive diagnosis of malignancy by the means of biopsy or histopathological analysis after surgery.

4. Outcome: diagnostic performance of the different diagnostic methods for the detection of (pre) cancerous lesions.

Studies were excluded from systematic review owing to the following reasons:

1. Not possible to determine sensitivity and specificity from the studies by means of reported true positive, true negative, false positive and true negative rates.

2. Case report, review and conference abstracts.

\section{Risk of bias}

The QUADAS-2 Tool was used to evaluate the quality of each eligible study ${ }^{29}$. The entire scale constituted 4 domains for the risk of bias: patient selection, index test, reference standard, and flow and timing. Additionally, there were 3 domains for applicability concerns: patient selection, index test, and reference standard. Each domain was judged for 3 levels of bias: low risk, intermediate/unclear risk, or high risk of bias. Full assessment criteria can be found in Supplemental Appendix 2 in the online version. 


\section{Classifications}

Ultrasound, mammogram and MRI were classified according to the Breast Imaging Reporting and Data System (BI-RADS) reporting system ${ }^{30}$. BI-RADS I-III was considered benign, and BI-RADS IV-VI was considered malignant or suspicious for malignancy. When cytologic examination indicated atypical cells, it was considered suspicious for malignancy.

\section{Statistical analysis}

First, sensitivity, specificity, positive predicted value (PPV), negative predicted value (NPV), and diagnostic accuracy (DA; number of truly positive and truly negative results divided by the total number of patients) were calculated for each of the 5 diagnostic methods for the diagnosis of nipple discharge for each study. After this, pooled estimates of sensitivity, specificity, PPV, NPV, and DA were calculated for each of the 5 diagnostic methods using fixed-effects models. Heterogeneity among studies was quantified by I 2 analysis and tested by the Cochran chi-square tests. Second, statistical computing software and network packages were used to draw the network graphs. Each node represents a different diagnostic method in which the size of the node reflects the number of patients, and the thickness of the line connecting the nodes represents the amount of included studies. Third, traditional pairwise meta-analyses were performed to compare different diagnostic modalities. Fourth, Bayesian network meta-analyses using the Mantel-Haenszel method were performed to combine the evidence from direct and indirect comparisons. Fifth, the surface under the cumulative ranking curve (SUCRA) was used to calculate for each intervention's being a measure of comparative diagnostic performance. A higher SUCRA value means that the intervention is likely to be ranked better than the comparators. ${ }^{31}$ Additionally, the separate indirect from direct design evidence (SIDDE) method was used to test the local consistency assumption of the NMA. ${ }^{32,33}$ Finally, subgroup traditional pairwise meta-analysis was performed to compare ductoscopy to MRI in studies in which all participants had negative ultrasound and/or mammogram, to compare the added value of ductoscopy and MRI to conventional imaging. $\mathrm{P}<.05$ and $95 \%$ confidence intervals of odds ratios not containing 1 were considered statistically significant.

Comparison-adjusted funnel plots for the NMA were performed to detect the small study effects on data. The Egger, Begg-Mazumdar, and Thomson-Sharp tests were used to quantify and test for asymmetry. For the subgroup traditional meta-analysis, the Egger test was used to quantify asymmetry. ${ }^{34-36} \mathrm{P} \geq .05$ indicated insufficient evidence for asymmetry and therefore also for no small sample bias and no publication bias.

All calculations were performed by RStudio 1.2.5001 (with R x64 3.6.1) (https://rstudio. $\mathrm{com} /$ ). Additionally, the following statistical packages were used for all computations of the network meta-analysis (NMA) and traditional meta-analyses: meta, mada, metafhor, gemtc, mvmeta, and netmeta. Visualization of plots was done using the ggplot2 package 


\section{Results}

\section{Selected papers}

We followed the PRISMA NMA checklist of items to include when reporting a systematic review involving a NMA (Supplemental Appendix 4 in the online version).

A total of 2583 citations were identified by the search and, after removing duplicates, and 181 potentially eligible articles were retrieved in full text (Figure 1). Overall, 3764 patients in 36 studies with PND underwent ultrasound, mammogram, MRI, cytology, and/or ductoscopy and were analyzed with an average of 104.6 participants per study with standard deviation of 68.7 .
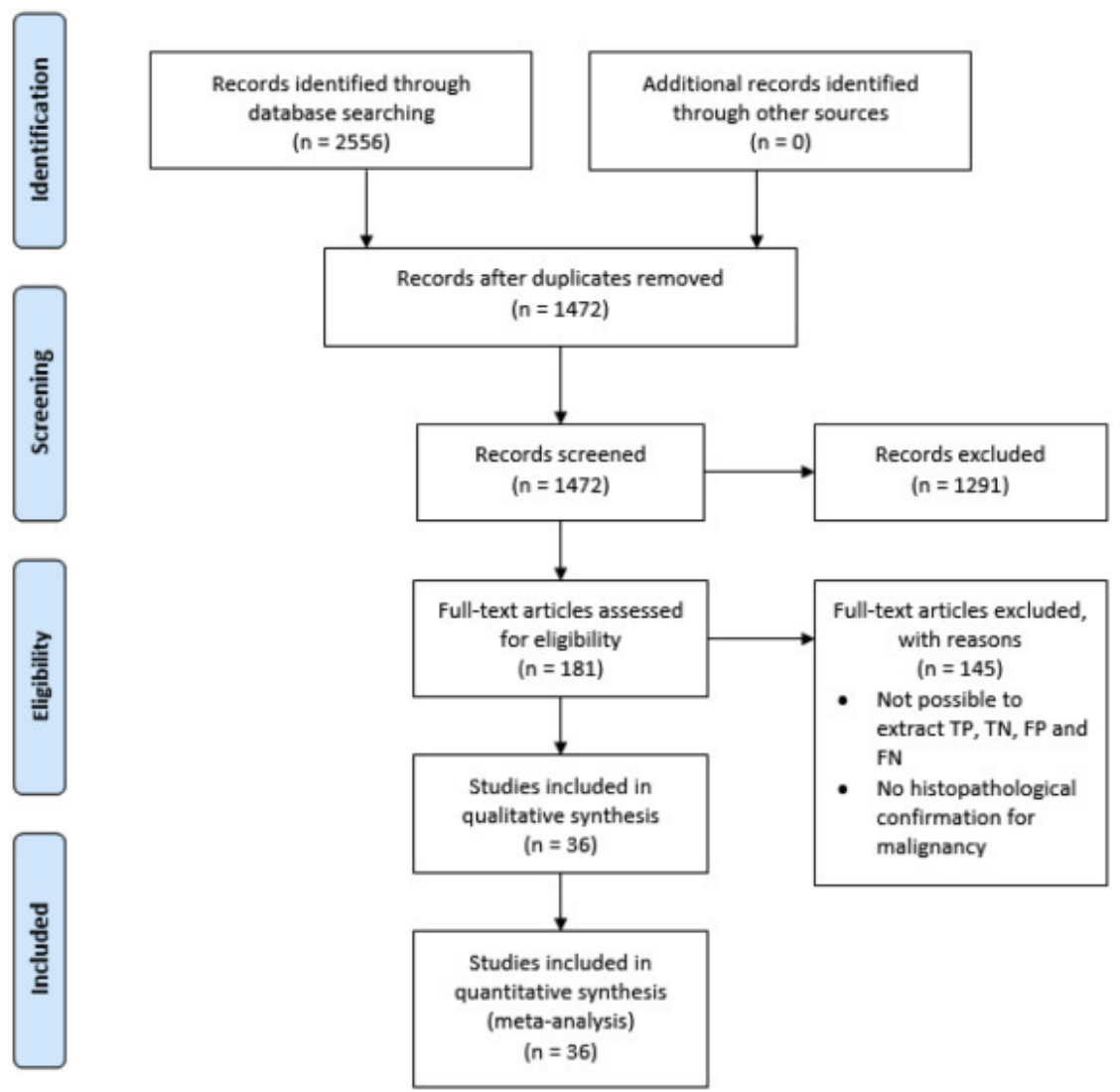

Figure 1. Flowchart Showing Literature Search and Study Selection. A Total of 36 Relevant Studies Were Ultimately Enrolled Into Our Network Meta-analysis on Diagnostic Approach to Pathologic Nipple Discharge Abbreviations: $\mathrm{FN}$ = falsely negative; $\mathrm{FP}=$ falsely positive; $\mathrm{TN}=$ truly negative; $\mathrm{TP}=$ truly positive. 
Figure 2 shows the network of eligible comparisons for sensitivity, specificity, NPV, PPV and DA of the different diagnostic methods. The single armed studies were not included in the network. Table 1 shows the studies included in the analysis and their characteristics.

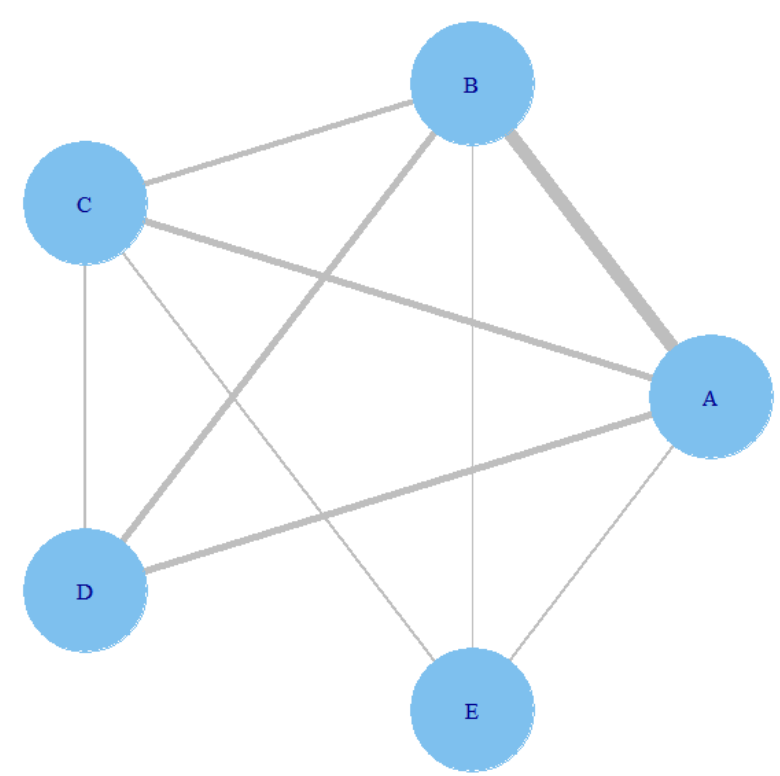

Figure 2. Evidence Network Plot of Diagnostic Value of Imaging Methods for Diagnosis of Pathologic Nipple Discharge. Imaging Methods Included (A) Ultrasound, (B) Mammogram, (C) Magnetic Resonance Imaging, (D) Cytology, and (E) Ductoscopy 
Table 1. Baseline characteristics of various diagnostic studies on pathologic nipple discharge

\begin{tabular}{|c|c|c|c|c|c|c|c|c|}
\hline \multirow[b]{2}{*}{ Author } & \multirow[b]{2}{*}{ year } & \multirow[b]{2}{*}{ country } & \multirow[b]{2}{*}{ gold standard } & \multirow[b]{2}{*}{$\mathbf{N}$} & \multicolumn{4}{|c|}{ Diagnostic modalities } \\
\hline & & & & & D1 & D2 & D3 & D4 \\
\hline Groves et al. $^{37}$ & 1996 & $\mathrm{UK}$ & histopathological diagnosis & 216 & $\mathrm{D}$ & & & \\
\hline Hou et al. ${ }^{38}$ & 2000 & Taiwan & histopathological diagnosis & 111 & $\mathrm{D}$ & & & \\
\hline Orel et al. ${ }^{39}$ & 2000 & USA & histopathological diagnosis & 15 & $\mathrm{C}$ & & & \\
\hline Hou et al. ${ }^{40}$ & 2002 & Taiwan & histopathological diagnosis & 176 & A & $\mathrm{B}$ & & \\
\hline Cabioglu et al. ${ }^{41}$ & 2003 & USA & histopathological diagnosis & 142 & A & B & $\mathrm{D}$ & \\
\hline Simmons et al. ${ }^{42}$ & 2003 & USA & histopathological diagnosis & 59 & A & $\mathrm{B}$ & $\mathrm{D}$ & \\
\hline Yamamoto et al. ${ }^{43}$ & 2003 & Japan & histopathological diagnosis & 60 & $\mathrm{D}$ & & & \\
\hline Moncrief et al. ${ }^{44}$ & 2005 & USA & histopathological diagnosis & 59 & $\mathrm{E}$ & & & \\
\hline Morrogh et al. ${ }^{45}$ & 2007 & USA & histopathological diagnosis & 33 & $\mathrm{C}$ & & & \\
\hline Denewer et al. ${ }^{46}$ & 2008 & Egypt & histopathological diagnosis & 53 & $\mathrm{E}$ & & & \\
\hline Bender et al. ${ }^{47}$ & 2009 & Turkey & histopathological diagnosis & 102 & $\mathrm{E}$ & & & \\
\hline Kooistra et al. ${ }^{13}$ & 2009 & Netherlands & histopathological diagnosis & 163 & $\mathrm{D}$ & & & \\
\hline Simpson et al. ${ }^{48}$ & 2009 & Canada & histopathological diagnosis & 39 & $\mathrm{E}$ & & & \\
\hline Tekin et al. ${ }^{49}$ & 2009 & Turkey & histopathological diagnosis & 34 & $\mathrm{D}$ & & & \\
\hline Vaughan et al. ${ }^{50}$ & 2009 & USA & histopathological diagnosis & 89 & $\mathrm{E}$ & & & \\
\hline Dolan et al. ${ }^{14}$ & 2010 & Ireland & histopathological diagnosis & 74 & $\mathrm{D}$ & & & \\
\hline Morrogh et al. ${ }^{51}$ & 2010 & USA & histopathological diagnosis & 270 & A & $\mathrm{B}$ & $\mathrm{C}$ & $\mathrm{D}$ \\
\hline Fisher et al. ${ }^{52}$ & 2011 & USA & histopathological diagnosis & 119 & $\mathrm{E}$ & & & \\
\hline Lorenzon et al. ${ }^{53}$ & 2011 & Italy & histopathological diagnosis & 38 & A & $\mathrm{B}$ & & \\
\hline Bahl et al. ${ }^{54}$ & 2015 & USA & histopathological diagnosis & 91 & $\mathrm{C}$ & & & \\
\hline Bahl et al. ${ }^{8}$ & 2015 & USA & histopathological diagnosis & 262 & A & B & & \\
\hline van Gelder et al. ${ }^{10}$ & 2015 & Netherlands & histopathological diagnosis & 107 & $\mathrm{C}$ & & & \\
\hline Waaijer et al. ${ }^{22}$ & 2015 & Netherlands & histopathological diagnosis & 53 & $\mathrm{E}$ & & & \\
\hline Zhao et al..$^{55}$ & 2015 & China & histopathological diagnosis & 153 & A & $\mathrm{D}$ & & \\
\hline Park et al. ${ }^{56}$ & 2016 & South Korea & histopathological diagnosis & 67 & A & & & \\
\hline Sanders et al. ${ }^{9}$ & 2016 & USA & histopathological diagnosis & 85 & $\mathrm{C}$ & & & \\
\hline Bahl et al. ${ }^{57}$ & 2017 & USA & histopathological diagnosis & 105 & $\mathrm{C}$ & & & \\
\hline Lesetedi et al. ${ }^{27}$ & 2017 & South Africa & histopathological diagnosis & 153 & A & $\mathrm{B}$ & & \\
\hline Yilmaz et al. ${ }^{58}$ & 2017 & Turkey & histopathological diagnosis & 26 & A & C & $\mathrm{E}$ & \\
\hline Gui et al. ${ }^{59}$ & 2018 & UK & histopathological diagnosis & 32 & $\mathrm{E}$ & & & \\
\hline Kan et al. ${ }^{60}$ & 2018 & China & histopathological diagnosis & 95 & A & B & $\mathrm{D}$ & \\
\hline Li et al. ${ }^{61}$ & 2018 & USA & histopathological diagnosis & 257 & A & B & $\mathrm{C}$ & $\mathrm{D}$ \\
\hline Baydoun et al. ${ }^{62}$ & 2019 & USA & histopathological diagnosis & 92 & A & B & & \\
\hline Jung et al. ${ }^{63}$ & 2019 & South Korea & histopathological diagnosis & 46 & A & & & \\
\hline Zacharioudakis et al. ${ }^{64}$ & 2019 & UK & histopathological diagnosis & 82 & $\mathrm{C}$ & & & \\
\hline Filipe et al. ${ }^{65}$ & 2020 & Netherlands & histopathological diagnosis & 206 & A & $\mathrm{B}$ & $\mathrm{C}$ & $\mathrm{E}$ \\
\hline
\end{tabular}

Diagnostic modalities are as follows: $\mathrm{A}=$ ultrasound; $\mathrm{B}=$ mammogram; $\mathrm{C}=$ magnetic resonance imaging (MRI); D = cytology; $\mathrm{E}=$ ductoscopy.

Abbreviations: $\mathrm{CI}=$ confidence interval; D1-4 = diagnostic methods. 


\section{Risks of bias}

The result of the QUADAS-2 tool revealed that all the included studies were of sufficient quality. This was for both risk-of-bias domains and applicability domains (Supplemental Figure 8 in the online version). Detailed information for each enrolled study can be found in Supplemental Figure 11 in the online version.

\section{Sensitivity, specificity, PPV, NPV and diagnostic accuracy}

Figure 3 summarizes the different ways of assessing accuracy for the detection of breast cancer in patients with PND. In terms of pooled sensitivity for the detection of malignity in patients with PND, MRI showed highest average sensitivity (83\%), followed by ductoscopy (58\%), ultrasound (50\%), cytology (38\%), and mammogram (22\%). Pooled specificity was highest for mammogram, at $93 \%$, followed by ductoscopy $(92 \%)$, cytology (90\%), MRI (76\%), and ultrasound (69\%). PPV was highest for mammogram (46\%), followed by ductoscopy (41\%), MRI (40\%), cytology $(39 \%)$, and ultrasound (31\%). Pooled NPV was highest for ductoscopy and MRI (both 96\%), followed by cytology (89\%), ultrasound (83\%), and mammogram (80\%). The highest DA was seen for ductoscopy (88\%), followed by cytology (82\%), MRI (77\%), mammogram (76\%), and ultrasound (65\%). Sensitivity, specificity, PPV, NPV, and DA of individual studies can be found in Supplemental Figure 1, Supplemental Figure 2, Supplemental Figure 3, Supplemental Figure 4, Supplemental Figure 5 in the online version. 


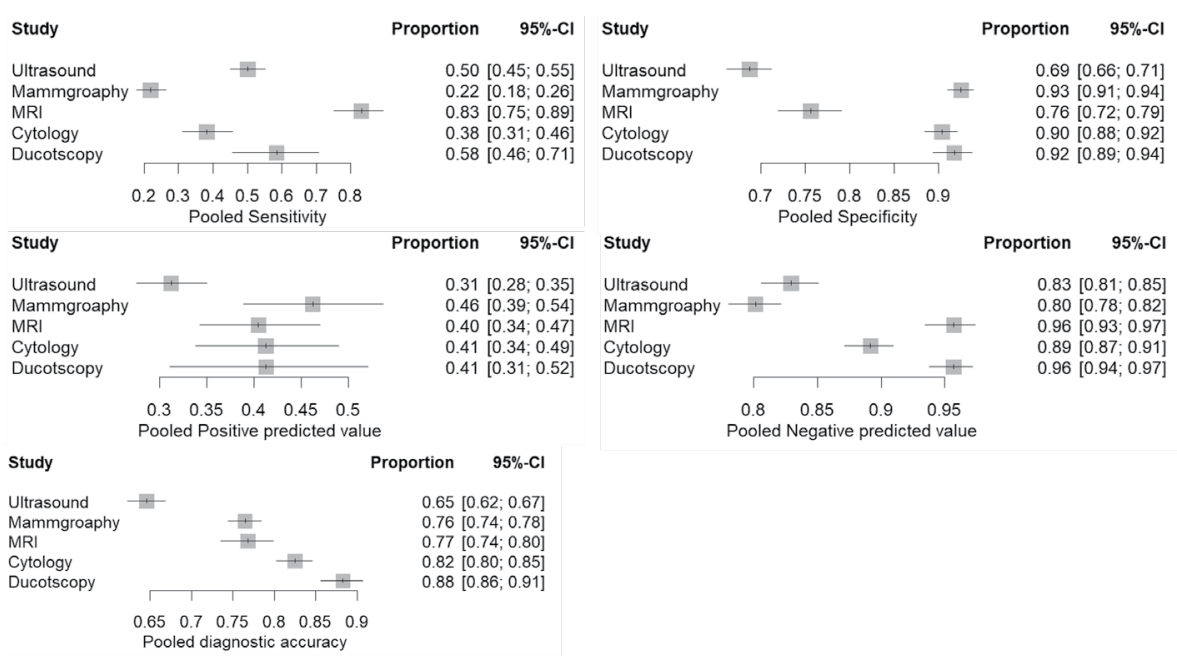

Figure 3. Usefulness of Approaches to Detection of Breast Cancer in Patients With Pathologic Nipple Discharge. Shown are Sensitivity, Specificity, Positive Predicted Value, Negative Predicted Value, and Diagnostic Accuracy of Different Approaches to Detection of Breast Cancer in Patients With Pathologic Nipple Discharge Abbreviations: $\mathrm{CI}=$ confidence interval; $\mathrm{MRI}=$ magnetic resonance imaging.

Pairwise meta-analysis from the literature (Table 2) showed mammogram to have statistically significantly lower sensitivity for the detection of malignancy in patients with PND than ultrasound, MRI, cytology, and ductoscopy. Additionally, ultrasound has a significantly lower sensitivity than MRI. Other combinations did not show statistically significant differences or were not directly measured in the currently available literature. Ultrasound showed significantly higher specificity than MRI, cytology, and mammogram. No statistical differences in specificity between ductoscopy and ultrasound were found. Mammogram has a statistically significantly higher PPV than ultrasound, MRI, and cytology. No direct comparisons in the literature were found between mammogram and ductoscopy. No other combinations showed statistical differences in PPV between the other diagnostic methods for the detection of breast cancer in patients with PND. NPV of MRI was statistically significantly higher than ultrasound and mammogram. No other comparisons between the 5 diagnostic methods showed any significant differences, although no direct comparison was available between ductoscopy and cytology. 
Table 2. Pairwise meta-analysis (in odds ratios (OR)) of five diagnostic methods to detect breast cancer in patients with pathologic nipple discharge

\begin{tabular}{cccccccccc}
\hline \multicolumn{1}{c}{ Sensitivity } & \multicolumn{1}{c}{ Specificity } \\
\hline $\begin{array}{c}\text { Studies } \\
(\mathrm{N})\end{array}$ & comparisons & OR & $\begin{array}{c}2.5 \% \\
\text { CI }\end{array}$ & $\begin{array}{c}97.5 \% \\
\text { CI }\end{array}$ & $\begin{array}{c}\text { Studies } \\
(\mathrm{N})\end{array}$ & comparisons & OR & $\begin{array}{c}2.5 \% \\
\text { CI }\end{array}$ & $\begin{array}{c}97.5 \% \\
\text { CI }\end{array}$ \\
\hline 10 & A vs B $^{\mathrm{b}}$ & 3.274 & 2.307 & 4.647 & 10 & A vs B $^{\mathrm{b}}$ & 0.185 & 0.141 & 0.243 \\
5 & A vs C $^{\mathrm{b}}$ & 0.337 & 0.137 & 0.828 & 5 & A vs C $^{\mathrm{b}}$ & 1.475 & 1.031 & 2.11 \\
5 & A vs D & 0.991 & 0.573 & 1.715 & 5 & A vs D & 0.982 & 0.650 & 1.484 \\
2 & A vs E & 0.188 & 0.02 & 1.796 & 2 & A vs E & 3.623 & 0.685 & 19.158 \\
4 & B vs C & 0.044 & 0.015 & 0.130 & 4 & B vs C & 19.532 & 11.903 & 32.051 \\
5 & B vs D & 0.449 & 0.249 & 0.808 & 5 & B vs D & 2.059 & 1.404 & 3.02 \\
$1^{\mathrm{a}}$ & B vs E & & & & 1 & B vs E & 2.043 & 0.337 & 12.386 \\
2 & C vs D & 2.143 & 0.583 & 7.871 & 2 & C vs D & 0.646 & 0.33 & 1.262 \\
2 & C vs E & 0.562 & 0.046 & 6.806 & 2 & C vs E & 1.46 & 0.265 & 8.036 \\
0 & D vs E & & & & 0 & D vs E & & &
\end{tabular}

\begin{tabular}{cccccccccc}
\hline & \multicolumn{3}{c}{ Positive predictive value } & \multicolumn{5}{c}{ Negative predictive value } \\
\hline $\begin{array}{c}\text { Studies } \\
(\mathrm{N})\end{array}$ & comparisons & OR & $\begin{array}{c}2.5 \% \\
\text { CI }\end{array}$ & $\begin{array}{c}97.5 \% \\
\text { CI }\end{array}$ & $\begin{array}{c}\text { Studies } \\
(\mathrm{N})\end{array}$ & comparisons & OR & $2.5 \%$ & $\begin{array}{c}97.5 \% \\
\text { CI }\end{array}$ \\
\hline 10 & A vs B $\mathbf{b}^{\mathbf{b}}$ & $\mathbf{0 . 3 9 8}$ & $\mathbf{0 . 2 6 2}$ & $\mathbf{0 . 6 0 6}$ & $\mathbf{1 0}$ & A vs B & $\mathbf{1 . 0 2 3}$ & $\mathbf{0 . 8 1 8}$ & $\mathbf{1 . 2 7 8}$ \\
5 & A vs C & $\mathbf{0 . 7 1 9}$ & $\mathbf{0 . 4 5}$ & $\mathbf{1 . 1 4 8}$ & $\mathbf{5}$ & A vs C $^{\mathbf{b}}$ & $\mathbf{0 . 4 0 2}$ & $\mathbf{0 . 1 6 2}$ & $\mathbf{0 . 9 9 9}$ \\
5 & A vs D & $\mathbf{0 . 9 3 3}$ & $\mathbf{0 . 5 2 9}$ & $\mathbf{1 . 6 4 5}$ & $\mathbf{5}$ & A vs D & $\mathbf{0 . 3 2 0}$ & $\mathbf{0 . 2 0 3}$ & $\mathbf{0 . 5 0 5}$ \\
2 & A vs E & $\mathbf{1 . 4 0 6}$ & $\mathbf{0 . 2 0 6}$ & $\mathbf{9 . 6 1 9}$ & $\mathbf{2}$ & A vs E & $\mathbf{0 . 3 2 6}$ & $\mathbf{0 . 0 8 5}$ & $\mathbf{1 . 2 5 2}$ \\
4 & B vs C b & $\mathbf{3 . 3 6 9}$ & $\mathbf{1 . 8 3 3}$ & $\mathbf{6 . 1 9 2}$ & $\mathbf{4}$ & B vs C & $\mathbf{0 . 3 3 9}$ & $\mathbf{0 . 1 4 2}$ & $\mathbf{0 . 8 1 0}$ \\
5 & B vs D & $\mathbf{2 . 5 5 1}$ & $\mathbf{1 . 3 5 8}$ & $\mathbf{4 . 7 9 2}$ & 5 & B vs D & 1.079 & 0.642 & 1.813 \\
$1^{\text {a }}$ & B vs E & & & & 1 & B vs E & 0.303 & 0.064 & 1.422 \\
2 & C vs D & 0.893 & 0.416 & 1.918 & 2 & C vs D & 1.867 & 0.515 & 6.765 \\
2 & C vs E & 1.688 & 0.249 & 11.416 & 2 & C vs E & 0.678 & 0.100 & 4.586 \\
0 & D vs E & & & & 0 & D vs E & & & \\
\hline
\end{tabular}

Diagnostic modalities are as follows: $\mathrm{A}=$ ultrasound; $\mathrm{B}=$ mammogram; $\mathrm{C}=$ magnetic resonance imaging (MRI); D = cytology; $\mathrm{E}=$ ductoscopy.

Abbreviations: $\mathrm{CI}=$ confidence interval; $\mathrm{OR}=$ odds ratio.

${ }^{\text {a }}$ Truly positive values were 0 , so it was not possible to pairwise compare sensitivity and positive predictive value.

${ }^{\text {B }}$ Statistically significant 


\section{Overall network meta-analysis}

The estimates resulting from direct and indirect evidence of diagnostic methods for the detection of breast cancer in patients with PND are provided in Table 3. Sensitivity of MRI was significantly superior to ultrasound, mammogram, and cytology but did not differ significantly from ductoscopy. Ductoscopy performed significantly better than mammogram, and ultrasound performed better than mammogram. Other comparisons did not show significant differences in sensitivity. Mammogram showed to be significantly more specific than all other diagnostic methods. Additionally, ultrasound was statistically inferior to cytology but superior to MRI for specificity. Furthermore, MRI was less specific than cytology. Ductoscopy did not differ significantly from cytology, MRI, and ultrasound with regard to specificity. PPV of ultrasound was inferior to mammogram but was not statistically different from other diagnostic methods. Mammogram had a significantly higher PPV than MRI but was similar to ductoscopy. Other combinations showed no statistically significant differences. NPV of ductoscopy did not differ significantly from the other diagnostic methods. MRI had significantly higher NPV than ultrasound and mammogram but was comparable to cytologic assessment. Finally, cytology had a higher NPV than mammogram. DA of ultrasound was significantly lower than mammogram and cytology but did not differ significantly from MRI and ductoscopy. MRI had a lower DA than mammogram and cytology but did not differ significantly from ductoscopy. All other comparisons did not show significant differences (Supplemental Table 1 in the online version). 
Table 3. Network meta-analysis comparing (in odds ratios (OR)) five diagnostic methods to detect breast cancer in patients with pathologic nipple discharge.

\begin{tabular}{|c|c|c|c|c|c|c|c|}
\hline \multicolumn{4}{|c|}{ Sensitivity } & \multicolumn{4}{|c|}{ Specificity } \\
\hline Comparisons & OR & $2.5 \% \mathrm{CI}$ & $97.5 \% \mathrm{CI}$ & comparisons & OR & $2.5 \% \mathrm{CI}$ & $97.5 \% \mathrm{CI}$ \\
\hline$A$ vs $B^{a}$ & 3.9212 & 2.7396 & 5.6123 & $A$ vs $B^{a}$ & 0.2269 & 0.1708 & 0.3013 \\
\hline$A$ vs $C^{a}$ & 0.2873 & 0.1181 & 0.6989 & A vs $C^{a}$ & 1.8271 & 1.2836 & 2.6008 \\
\hline A vs D & 1.3188 & 0.7653 & 2.2728 & A vs D & 0.7291 & 0.493 & 1.0783 \\
\hline A vs $E$ & 0.1787 & 0.0197 & 1.6187 & A vs $E$ & 1.6403 & 0.4712 & 5.7103 \\
\hline $\mathrm{B}$ vs $\mathrm{C}^{\mathrm{a}}$ & 0.0733 & 0.0297 & 0.1808 & $\mathrm{~B}$ vs $\mathrm{C}^{\mathrm{a}}$ & 8.0542 & 5.3315 & 12.1672 \\
\hline $\mathrm{B}$ vs $\mathrm{D}^{\mathrm{a}}$ & 0.3363 & 0.1918 & 0.5898 & $\mathrm{~B}$ vs $\mathrm{D}^{\mathrm{a}}$ & 3.2138 & 2.1276 & 4.8545 \\
\hline$B$ vs $E^{a}$ & 0.0456 & 0.0049 & 0.4218 & $\mathrm{~B}$ vs $\mathrm{E}^{\mathrm{a}}$ & 7.2304 & 2.0646 & 25.321 \\
\hline$C$ vs $D^{a}$ & 4.5908 & 1.6963 & 12.4242 & $\mathrm{C}$ vs $\mathrm{D}^{\mathrm{a}}$ & 0.399 & 0.2442 & 0.6521 \\
\hline C vs E & 0.6219 & 0.0645 & 5.9959 & C vs E & 0.8977 & 0.2547 & 3.1637 \\
\hline $\mathrm{D}$ vs $\mathrm{E}$ & 0.1355 & 0.0141 & 1.3025 & D vs $\mathrm{E}$ & 2.2498 & 0.6165 & 8.21 \\
\hline \multicolumn{4}{|c|}{ Positive predictive value } & \multicolumn{4}{|c|}{ Negative predictive value } \\
\hline comparisons & OR & $2.5 \% \mathrm{CI}$ & $97.5 \% \mathrm{CI}$ & comparisons & OR & $2.5 \% \mathrm{CI}$ & $97.5 \% \mathrm{CI}$ \\
\hline$A$ vs $B^{a}$ & 0.3942 & 0.2574 & 0.6038 & $A$ vs $B$ & 0.9272 & 0.7309 & 1.1762 \\
\hline A vs $\mathrm{C}$ & 0.8313 & 0.5249 & 1.3167 & A vs $C^{a}$ & 0.3108 & 0.1333 & 0.7245 \\
\hline A vs D & 0.8627 & 0.4979 & 1.4947 & A vs $D^{a}$ & 0.5127 & 0.3285 & 0.8000 \\
\hline A vs $E$ & 1.4045 & 0.2756 & 7.1581 & A vs $E$ & 0.3017 & 0.0830 & 1.0966 \\
\hline $\mathrm{B}$ vs $\mathrm{C}^{\mathrm{a}}$ & 2.1088 & 1.2226 & 3.6374 & $\mathrm{~B}$ vs $\mathrm{C}^{\mathrm{a}}$ & 0.3352 & 0.1470 & 0.7641 \\
\hline $\mathrm{B}$ vs $\mathrm{D}^{\mathrm{a}}$ & 2.1883 & 1.2002 & 3.9899 & $\mathrm{~B}$ vs $\mathrm{D}^{\mathrm{a}}$ & 0.5529 & 0.3500 & 0.8736 \\
\hline $\mathrm{B}$ vs $\mathrm{E}$ & 3.5629 & 0.6714 & 18.907 & $B$ vs $E$ & 0.3254 & 0.0894 & 1.1844 \\
\hline C vs D & 1.0377 & 0.5458 & 1.9728 & C vs D & 1.6496 & 0.6507 & 4.1822 \\
\hline C vs E & 1.6895 & 0.3317 & 8.6049 & C vs E & 0.9708 & 0.2232 & 4.2221 \\
\hline D vs $E$ & 1.6282 & 0.2963 & 8.9474 & D vs $E$ & 0.5885 & 0.1516 & 2.2854 \\
\hline
\end{tabular}

Diagnostic modalities are as follows: $\mathrm{A}=$ ultrasound; $\mathrm{B}=$ mammogram; $\mathrm{C}=$ magnetic resonance imaging (MRI); D = cytology; $\mathrm{E}=$ ductoscopy.

Abbreviations: $\mathrm{CI}=$ confidence interval; $\mathrm{OR}=$ odds ratio.

a Statistically significant. 
SUCRA values indicating the probability of having the best diagnostic performance are shown in Supplemental Table 2 in the online version. The highest SUCRA value for sensitivity was seen for mammogram and the lowest for ductoscopy. The highest SUCRA value for specificity was seen for MRI, followed by ductoscopy, with the lowest seen for mammogram. Ductoscopy showed the highest SUCRA value for PPV and mammogram the lowest. Ultrasound showed the highest SUCRA value for NPV and the MRI the lowest. The highest SUCRA values for DA were seen for ultrasound and the lowest for cytology. Finally, the SIDDE approach did not provide strong evidence for inconsistency with regard to sensitivity, specificity, PPV, NPV, and DA for all comparisons (all P > .182).

\section{Subgroup meta-analysis}

Subgroup analysis comparing sensitivity, specificity, PPV, and NPV of MRI and ductoscopy when conventional imaging (ultrasound and mammogram) is negative can be found in Figure 4 and Table 4. Pooled sensitivity of 5 remaining studies each was $44 \%$ for ductoscopy and $76 \%$ for MRI (not significant). Specificity and DA were significantly higher for ductoscopy compared to MRI (98\% vs. $84 \%$, 95\%-83\%, respectively). PPV and NPV were insignificant between ductoscopy and MRI. Individual diagnostic performance of each study can be found in Supplemental Figures 6 and 7 in the online version for, respectively, ductoscopy and MRI.

\section{Assessment of publication bias}

The results of assessment of publication bias showed symmetrical distributions for sensitivity, specificity, PPV, NPV, and DA. This indicates that there is no evidence to conclude small sample effects or publication bias in this NMA (Supplemental Figure 9 in the online version). Subgroup analysis also showed symmetry of the effect (Supplemental Figure 10 in the online version). For the subgroup analyses, $\mathrm{P}$ values for the Egger test for sensitivity, specificity, PPV, NPV, and DA were 0.0504, 0.7549, 0.8378, 0.2211, and 0.8187 , respectively. 


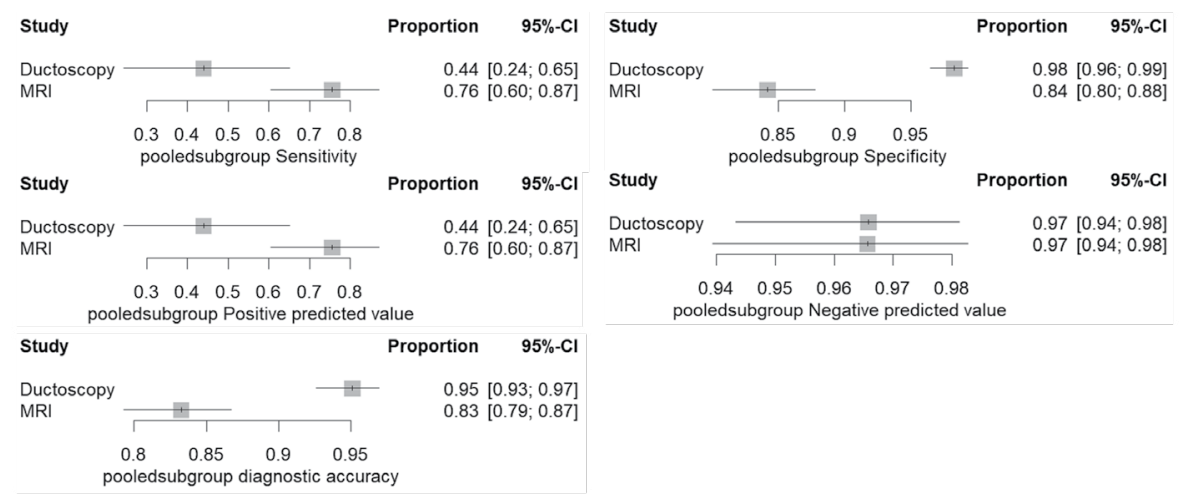

Figure 4. Usefulness of Approaches to Detection of Breast Cancer in Patients With Pathologic Nipple Discharge and Negative Imaging Results. Shown are Pooled Sensitivity, Specificity, Positive Predicted Value, Negative Predicted Value, and Diagnostic Accuracy of Different Approaches to Detection of Breast Cancer in Subgroup of Patients With Pathologic Nipple Discharge and With Negative Mammogram And/Or Ultrasound Results Abbreviations: $\mathrm{CI}=$ confidence interval; $\mathrm{MRI}=$ magnetic resonance imaging. 


\section{Discussion}

There is no consensus on the diagnostic approach of patients with PND, especially if conventional imaging (ultrasound and mammogram) results are negative. High-quality studies on the value of ductoscopy and MRI are scarce. The first aim of this study was to compare by means of NMA the effectiveness for detection of malignancy of ultrasound, mammogram, MRI, cytology, of nipple fluid and ductoscopy in patients with PND. The second aim of this study was to compare MRI to ductoscopy when ultrasound and mammogram as conventional first-line imaging techniques have negative results.

The online search yielded 2556 hits, of which 36 studies with 3764 patients were included. The highest sensitivity was seen for MRI (83\%) and ductoscopy (58\%), and the highest specificity was seen for ductoscopy (92\%) and mammogram (93\%), whereas MRI had a relatively low specificity $(76 \%)$. DA was highest for ductoscopy $(88 \%)$, followed by cytology $(82 \%)$ and MRI (77\%). In subgroup analysis including studies in which patients had negative ultrasound and mammogram, there were no statistical differences in sensitivity, but specificity and DA were significantly higher for ductoscopy.

Ultrasound and mammography are established cheap breast imaging methods. ${ }^{66,67}$ We found that the pooled average sensitivity for the detection of breast cancer in patients with PND was $50 \%$ for ultrasound but only $22 \%$ for mammogram. Pooled specificity was $69 \%$ for ultrasound but much was higher, at 93\%, for mammogram. Most causes of PND (around 95\%), such as papillomas and ductal ectasia, are benign, ${ }^{6,7}$ for which ultrasound ${ }^{5,8,12,63,68}$ and mammography 5,12,51,60,68 indeed have a high sensitivity and specificity. Therefore, ultrasound and mammography are likely to remain the initial approach in patients with PND.

MRI has been used more often for the detection of breast cancer in patients with PND in recent years. We found that the pooled average sensitivity of MRI is $86 \%$ and specificity is $76 \%$. Pooled PPV of MRI was low $(40 \%)$, meaning that over half of patients with a positive MRI are advised to undergo histopathologic analysis by core biopsy and/or surgery for a benign lesion (Supplemental Figure 3 in the online version). ${ }^{10,11}$ Therefore, MRI may need to be reserved for PND cases where mammogram and ultrasound are negative. ${ }^{8,10,54,64}$ The current NMA shows that the sensitivity of MRI then drops to $74 \%$ but specificity increases to $85 \%$ (Supplemental Figure 7). Contrast-enhanced MRI appears to be a promising approach for the detection of breast cancer in patients with PND in pilot studies. (50-55)

For cytology, pooled sensitivity was only $38 \%$, although the pooled specificity was high $(90 \%)$ (Supplemental Figure 4), indicating that cytology is not very useful for the detection of breast cancer in patients with PND. ${ }^{13}$ However, biomarker analysis of nipple discharge, as by RNA assessment, ${ }^{69}$ may be more promising ${ }^{6,70-77}$.

This NMA showed that ductoscopy has an average sensitivity of $58 \%$ and a high specificity of $92 \%$ for the detection of breast cancer in patients with PND. However, ductoscopy is 
highly suitable for detecting benign lesions causing PND. ${ }^{46,47,50,59,78,79}$ However, without histologic sampling of the lesions found, ductoscopy images alone cannot permit reliable discrimination between benign and malignant causes; endoscopic sampling during ductoscopy is not possible; and surgery may still be warranted to exclude malignancy. ${ }^{24}$ Ductoscopy is not a cheap technique, like MRI, so it may be especially useful when conventional imaging is negative. ${ }^{65}$ For this reason, we conducted a subgroup metaanalysis comparing MRI to ductoscopy in patients with PND by negative conventional imaging for the detection of breast cancer. Sensitivity for ductoscopy dropped to $44 \%$ but specificity rose to $98 \%$, which is significantly higher than for MRI. However, the low incidence of malignancy in patients with PND leads to broad confidence intervals, meaning that the pooled relative difference in sensitivity is relatively big, but not statistically significant. Moreover, the higher incidence of malignancy in the MRI studies (around $20 \%$ vs. the $4.5 \%$ in the ductoscopy studies) may explain the relatively high sensitivity of MRI, which may therefore not be realistic. This high incidence of malignancy in MRI studies is unexpected because the reported incidence of malignancy in patients with negative echography and mammogram is around $5 \% .{ }^{26,27}$ The same applies for the PPV. Consequently, because the most common causes of PND are benign, specificity may be clinically more relevant. The present study found that ductoscopy has a statistically significantly higher specificity (and DA) than MRI, so it may be a more useful diagnostic tool in patients with PND with no signs of malignancy by conventional radiography. Additionally, intraductal biopsies are nowadays possible with the basket extraction device, ${ }^{22}$ and new techniques surrounding ductoscopy are being developed in order to increase the sensitivity for the detection of (pre)malignant lesions, such as autofluorescent imaging. ${ }^{80,81}$

To our knowledge, this is the first systematic review comparing different diagnostic methods for the detection of malignancy in patients with PND. However, there are some limitations of this NMA. This study could not provide enough direct comparisons between the 5 individual imaging methods as a result of limited evidence. Nonetheless, further research is warranted comparing availability, impact to the patient, and costeffectiveness of the different diagnostic methods. Finally, most women with PND undergo surgical procedures, such as microdochectomy or major duct excision, to exclude malignancy and treat the PND symptoms. ${ }^{6,8} 9$ These surgical procedures are performed under general anesthesia, are expensive, and are associated with scarring, which may result in breastfeeding difficulties. ${ }^{68}$ Additionally, heterogeneity was high within the groups and between different groups. We cannot explain the high heterogeneity because we consider the methodology and patient population of the studies to be very similar, especially in the subgroup analysis. Furthermore, we have no explanation for the high incidence of malignancy in the MRI studies.

To conclude, our findings suggest that although ultrasound and mammogram may remain low-cost useful first choices for the detection of malignancy in patients with PND, ductoscopy outperforms most imaging techniques (especially MRI) and cytology. 


\section{References}

1. Hussain AN, Policarpio C, Vincent MT. Evaluating nipple discharge. Obstet Gynecol Surv. 2006;61(4):278-283. doi:0006254-200604000-00025 [pii]

2. Dixon JM, Mansel RE. ABC of breast diseases. Symptoms assessment and guidelines for referral. BMJ. 1994;309(6956):722-726.

3. Seltzer MH. Breast complaints, biopsies, and cancer correlated with age in 10,000 consecutive new surgical referrals. Breast J. 2004;10(2):111-117. doi:21284 [pii]

4. Santen RJ, Mansel R. Benign breast disorders. N Engl J Med. 2005;353(3):275-285. doi:353/3/275 [pii]

5. King TA, Carter KM, Bolton JS, Fuhrman GM. A simple approach to nipple discharge. Am Surg. 2000;66(10):960-966.

6. Alcock C, Layer GT. Predicting occult malignancy in nipple discharge. ANZ J Surg. 2010;80(9):646-649. doi:10.1111/j.1445-2197.2010.05270.x [doi]

7. Albrecht C, Thele F, Grunwald S, et al. Nipple discharge: role of ductoscopy in comparison with standard diagnostic tests. Onkologie. 2013;36(1-2):12-16. doi:10.1159/000346639 [doi]

8. Bahl M, Baker JA, Greenup RA, Ghate S V. Diagnostic Value of Ultrasound in Female Patients With Nipple Discharge. AJRAmerican J Roentgenol. 2015;205(1):203-208. doi:10.2214/AJR.14.13354 [doi]

9. Sanders LM, Daigle M. The Rightful Role of MRI after Negative Conventional Imaging in the Management of Bloody Nipple Discharge. Breast J. 2016;22(2):209-212. doi:10.1111/tbj.12551 [doi]

10. van Gelder L, Bisschops RH, Menke-Pluymers MB, Westenend PJ, Plaisier PW. Magnetic resonance imaging in patients with unilateral bloody nipple discharge; useful when conventional diagnostics are negative? World J Surg. 2015;39(1):184-186. doi:10.1007/s00268-014-2701-1 [doi]

11. on Breast Imaging: EP, Lee SJ, Trikha S, et al. ACR Appropriateness Criteria((R)) Evaluation of Nipple Discharge. J Am Coll Radiol. 2017;14(5S):S138-S153. doi:S1546-1440(17)30137-0 [pii]

12. Paula IB de, Campos AM. Breast imaging in patients with nipple discharge. Radiol Bras. 2017. doi: $10.1590 / 0100-3984.2016 .0103$

13. Kooistra BW, Wauters C, van de Ven S, Strobbe L. The diagnostic value of nipple discharge cytology in 618 consecutive patients. Eur J Surg Oncol. 2009;35(6):573-577. doi:10.1016/j.ejso.2008.09.009 [doi]

14. Dolan RT, Butler JS, Kell MR, Gorey TF, Stokes MA. Nipple discharge and the efficacy of duct cytology in evaluating breast cancer risk. Surgeon. 2010;8(5):252-258. doi:10.1016/j.surge.2010.03.005 [doi]

15. Yamamoto D, Shoji T, Kawanishi H, et al. A utility of ductography and fiberoptic ductoscopy for patients with nipple discharge. Breast Cancer Res Treat. 2001;70(2):103-108.

16. Yamamoto D, Ueda S, Senzaki H, et al. New diagnostic approach to intracystic lesions of the breast by fiberoptic ductoscopy. Anticancer Res. 2001;21(6A):4113-4116.

17. Okazaki A, Okazaki M, Asaishi K, et al. Fiberoptic ductoscopy of the breast: a new diagnostic procedure for nipple discharge. Jpn J Clin Oncol. 1991;21(3):188-193.

18. Matsunaga T, Ohta D, Misaka T, et al. Mammary ductoscopy for diagnosis and treatment of intraductal lesions of the breast. Breast Cancer. 2001;8(3):213-221.

19. Grunwald S, Heyer H, Paepke S, et al. Diagnostic value of ductoscopy in the diagnosis of nipple discharge and intraductal proliferations in comparison to standard methods. Onkologie. 2007;30(5):243-248. doi:100848 [pii] 
20. Han Y, Li J, Han S, Jia S, Zhang Y, Zhang W. Diagnostic value of endoscopic appearance during ductoscopy in patients with pathological nipple discharge. BMC Cancer. 2017;17(1):300-303. doi:10.1186/s12885017-3288-3 [doi]

21. Kamali S, Bender O, Kamali GH, Aydin MT, Karatepe O, Yuney E. Diagnostic and therapeutic value of ductoscopy in nipple discharge and intraductal proliferations compared with standard methods. Breast Cancer. 2014;21(2):154-161. doi:10.1007/s12282-012-0377-7 [doi]

22. Waaijer L, van Diest PJ, Verkooijen HM, et al. Interventional ductoscopy in patients with pathological nipple discharge. Br J Surg. 2015;102(13):1639-1648. doi:10.1002/bjs.9950 [doi]

23. Jacobs VR, Kiechle M, Plattner B, Fischer T, Paepke S. Breast ductoscopy with a 0.55-mm miniendoscope for direct visualization of intraductal lesions. J Minim Invasive Gynecol. 2005;12(4):359-364. doi:S1553-4650(05)00274-8 [pii]

24. Waaijer L, Simons JM, Borel Rinkes IH, van Diest PJ, Verkooijen HM, Witkamp AJ. Systematic review and meta-analysis of the diagnostic accuracy of ductoscopy in patients with pathological nipple discharge. Br J Surg. 2016;103(6):632-643. doi:10.1002/bjs.10125 [doi]

25. Makita M, Akiyama F, Gomi N, Iwase T. Mammary ductoscopy and watchful follow-up substitute microdochectomy in patients with bloody nipple discharge. Breast Cancer. 2016;23(2):242-251. doi:10.1007/s12282-014-0561-z [doi]

26. Galvin R, Joyce D, Downey E, Boland F, Fahey T, Hill AK. Development and validation of a clinical prediction rule to identify suspected breast cancer: a prospective cohort study. BMC Cancer. 2014;14:743. doi:10.1186/1471-2407-14-743 [doi]

27. Lesetedi C, Rayne S, Kruger D, Benn CA. Indicators of breast cancer in patients undergoing microdochectomy for a pathological nipple discharge in a middle-income country. J Surg Res. 2017;220:336-340. doi:S0022-4804(17)30428-6 [pii]

28. Hutton B, Salanti G, Caldwell DM, et al. The PRISMA extension statement for reporting of systematic reviews incorporating network meta-analyses of health care interventions: checklist and explanations. Ann Intern Med. 2015;162(11):777-784. doi:10.7326/M14-2385 [doi]

29. Whiting PF, Rutjes AW, Westwood ME, et al. QUADAS-2: a revised tool for the quality assessment of diagnostic accuracy studies. Ann Intern Med. 2011;155(8):529-536. doi:10.7326/0003-4819-155-8201110180-00009 [doi]

30. Rao AA, Feneis J, Lalonde C, Ojeda-Fournier H. A Pictorial Review of Changes in the BI-RADS Fifth Edition. Radiographics. 2016;36(3):623-639. doi:10.1148/rg.2016150178 [doi]

31. Salanti G, Ades AE, Ioannidis JP. Graphical methods and numerical summaries for presenting results from multiple-treatment meta-analysis: an overview and tutorial. J Clin Epidemiol. 2011;64(2):163-171. doi:10.1016/j.jclinepi.2010.03.016 [doi]

32. Efthimiou O, Debray TP, van Valkenhoef G, et al. GetReal in network meta-analysis: a review of the methodology. Res Synth Methods. 2016;7(3):236-263. doi:10.1002/jrsm.1195 [doi]

33. Efthimiou O, Rucker G, Schwarzer G, Higgins JPT, Egger M, Salanti G. Network meta-analysis of rare events using the Mantel-Haenszel method. Stat Med. 2019;38(16):2992-3012. doi:10.1002/ $\operatorname{sim} .8158$ [doi]

34. Egger M, Smith GD, Schneider M, Minder C. Bias in meta-analysis detected by a simple, graphical test. BMJ. 1997;315(7109):629-634. doi:10.1136/bmj.315.7109.629 [doi]

35. Begg CB, Mazumdar M. Operating characteristics of a rank correlation test for publication bias. Biometrics. 1994;50(4):1088-1101. 
36. Thompson SG, Sharp SJ. Explaining heterogeneity in meta-analysis: a comparison of methods. Stat Med. 1999;18(20):2693-2708. doi:10.1002/(SICI)1097-0258(19991030)18:20<2693::AIDSIM235>3.0.CO;2-V [pii]

37. Groves AM, Carr M, Wadhera V, Lennard TWJJ. An audit of cytology in the evaluation of nipple discharge . A retrospective study of 10 years ' experience. Breast. 1996;5(2):96-99. doi:10.1016/S09609776(96)90129-0

38. Hou M, Tsai K, Lin H, Chai C, Liu C, Huang T. A simple intraductal aspiration method for cytodiagnosis in nipple discharge. Acta Cytol. 2000;44(6):1029-1034. doi:10.1159/000328592 [doi]

39. Orel SG, Dougherty CS, Reynolds C, Czerniecki BJ, Siegelman ES, Schnall MD. MR imaging in patients with nipple discharge: initial experience. Radiology. 2000;216(1):248-254. doi:10.1148/ radiology.216.1.r00jn28248 [doi]

40. Hou MF, Tsai KB, Ou-Yang F, et al. Is a one-step operation for breast cancer patients presenting nipple discharge without palpable mass feasible? Breast. 2002;11(5):402-407. doi:10.1054/brst.2002.0441 [doi]

41. Cabioglu N, Hunt KK, Singletary SE, et al. Surgical decision making and factors determining a diagnosis of breast carcinoma in women presenting with nipple discharge. J Am Coll Surg. 2003;196(3):354-364. doi:S1072-7515(02)01606-X [pii]

42. Simmons R, Adamovich T, Brennan M, et al. Nonsurgical evaluation of pathologic nipple discharge. Ann Surg Oncol. 2003;10(2):113-116. doi:10.1245/aso.2003.03.089 [doi]

43. Yamamoto D, Senzaki H, Nakagawa H, Okugawa H, Gondo H, Tanaka K. Detection of chromosomal aneusomy by fluorescence in situ hybridization for patients with nipple discharge. Cancer. 2003;97(3):690694. doi:10.1002/cncr.11091 [doi]

44. Moncrief RM, Nayar R, Diaz LK, Staradub VL, Morrow M, Khan SA. A comparison of ductoscopyguided and conventional surgical excision in women with spontaneous nipple discharge. Ann Surg. 2005;241(4):575-581. doi:00000658-200504000-00006 [pii]

45. Morrogh M, Morris EA, Liberman L, Borgen PI, King TA. The predictive value of ductography and magnetic resonance imaging in the management of nipple discharge. Ann Surg Oncol. 2007;14(12):33693377. doi:10.1245/s10434-007-9530-5 [doi]

46. Denewer A, El-Etribi K, Nada N, El-Metwally M. The role and limitations of mammary ductoscope in management of pathologic nipple discharge. Breast J. 2008;14(5):442-449. doi:10.1111/j.15244741.2008.00620.x [doi]

47. Bender O, Balci FL, Yuney E, Akbulut H. Scarless endoscopic papillomectomy of the breast. Onkologie. 2009;32(3):94-98. doi:10.1159/000195694 [doi]

48. Simpson JS, Connolly EM, Leong WL, et al. Mammary ductoscopy in the evaluation and treatment of pathologic nipple discharge: a Canadian experience. Can J surgeryJournal Can Chir. 2009;52(6):E245-8.

49. Tekin E, Akin M, Kurukahvecioglu O, et al. The value of breast ductoscopy in radiologically negative spontaneous/persistent nipple discharge. Breast J. 2009;15(4):329-332. doi:10.1111/j.15244741.2009.00735.x [doi]

50. Vaughan A, Crowe JP, Brainard J, Dawson A, Kim J, Dietz JR. Mammary ductoscopy and ductal washings for the evaluation of patients with pathologic nipple discharge. Breast J. 2009;15(3):254-260. doi: 10.1111/j.1524-4741.2009.00714.x [doi]

51. Morrogh M, Park A, Elkin EB, King TA. Lessons learned from 416 cases of nipple discharge of the breast. Am J Surg. 2010;200(1):73-80. doi:10.1016/j.amjsurg.2009.06.021 [doi] 
52. Cyr AE, Margenthaler JA, Conway J, et al. Correlation of ductal lavage cytology with ductoscopy-directed duct excision histology in women at high risk for developing breast cancer: a prospective, single-institution trial. Ann Surg Oncol. 2011;18(11):3192-3197. doi:10.1245/s10434-011-1963-1 [doi]

53. Lorenzon M, Zuiani C, Linda A, Londero V, Girometti R, Bazzocchi M. Magnetic resonance imaging in patients with nipple discharge: should we recommend it? Eur Radiol. 2011;21(5):899-907. doi:10.1007/ s00330-010-2009-y [doi]

54. Bahl M, Baker JA, Greenup RA, Ghate S V. Evaluation of Pathologic Nipple Discharge: What is the Added Diagnostic Value of MRI? Ann Surg Oncol. 2015;22 Suppl 3:S435-41. doi:10.1245/s10434-0154792-9 [doi]

55. Zhao S, Gai X, Wang Y, et al. Diagnostic Values of Carcinoembryonic Antigen, Cancer Antigen 15-3 and Cancer Antigen 125 Levels in Nipple Discharge. Chin J Physiol. 2015;58(6):385-392. doi:10.4077/ CJP.2015.BAD336 [doi]

56. Park CJ, Kim EK, Moon HJ, Yoon JH, Kim MJ. Reliability of Breast Ultrasound BI-RADS Final Assessment in Mammographically Negative Patients with Nipple Discharge and Radiologic Predictors of Malignancy. J Breast Cancer. 2016;19(3):308-315. doi:10.4048/jbc.2016.19.3.308 [doi]

57. Bahl M, Gadd MA, Lehman CD. JOURNAL CLUB: Diagnostic Utility of MRI After Negative or Inconclusive Mammography for the Evaluation of Pathologic Nipple Discharge. AJRAmerican J Roentgenol. 2017;209(6):1404-1410. doi:10.2214/AJR.17.18139 [doi]

58. Yilmaz R, Bender O, Yabul FC, Dursun M, Tunaci M, Acunas G. Diagnosis of Nipple Discharge: Value of Magnetic Resonance Imaging and Ultrasonography in Comparison with Ductoscopy. Balkan Med J. 2017;34(2):119-126. doi:10.4274/balkanmedj.2016.0184 [doi]

59. Gui G, Agusti A, Twelves D, et al. INTEND II randomized clinical trial of intraoperative duct endoscopy in pathological nipple discharge. Br J Surg. 2018;105(12):1583-1590. doi:10.1002/bjs.10990 [doi]

60. Kan WM, Chen C, Kwong A. Implications of nipple discharge in Hong Kong Chinese women. Hong Kong Med J = Xianggang yi xue za zhi. 2018;24(1):18-24. doi:10.12809/hkmj154764 [doi]

61. Li GZ, Wong SM, Lester S, Nakhlis F. Evaluating the risk of underlying malignancy in patients with pathologic nipple discharge. Breast J. 2018;24(4):624-627. doi:10.1111/tbj.13018 [doi]

62. Baydoun S, Gonzalez P, Whitman GJ, Dryden M, Xi Y, Dogan B. Is Ductography Still Warranted in the 21st century? Breast J. 2019;25(4):654-662. doi:10.1111/tbj.13302 [doi]

63. Jung HK, Park YM, Baek HJ, et al. Comparison Between Ultrasonography and Galactography in Detecting Lesions in Patients With Pathologic Nipple Discharge. Ultrasound Q. 2019;35(1):93-98. doi:10.1097/RUQ.0000000000000365 [doi]

64. Zacharioudakis K, Kontoulis T, Vella JX, et al. Can we see what is invisible? The role of MRI in the evaluation and management of patients with pathological nipple discharge. Breast Cancer Res Treat. 2019;178(1):115-120. doi:10.1007/s10549-019-05321-w [doi]

65. Filipe MD, Waaijer L, van der Pol CC, van Diest P, Witkamp AJ. Interventional ductoscopy as an alternative for major duct excision or microdochectomy in women suffering pathological nipple discharge: a single centre experience. Clin Breast Cancer. January 2020. doi:10.1016/j.clbc.2019.12.008

66. Health Quality Ontario. Ultrasound as an Adjunct to Mammography for Breast Cancer Screening: A Health Technology Assessment. Ont Health Technol Assess Ser. 2016;16(15):1-71. http://www. pubmedcentral.nih.gov/articlerender.fcgi?artid=PMC4947971. Accessed February 20, 2020.

67. Mandrik O, Ekwunife OI, Meheus F, et al. Systematic reviews as a "lens of evidence": Determinants of cost-effectiveness of breast cancer screening. Cancer Med. 2019;8(18):7846-7858. doi:10.1002/ cam4.2498 
68. Sarakbi W Al, Worku D, Escobar PF, Mokbel K. Breast papillomas: current management with a focus on a new diagnostic and therapeutic modality. Int Semin Surg Oncol. 2006;3:1. doi:1477-7800-3-1 [pii]

69. Zhang K, Zhao S, Wang Q, Yang HS, Zhu J, Ma R. Identification of microRNAs in Nipple Discharge as Potential Diagnostic Biomarkers for Breast Cancer. Ann Surg Oncol. 2015;22 Suppl 3:S536-44. doi:10.1245/s10434-015-4586-0 [doi]

70. Zhao S, Mei Y, Wang J, Zhang K, Ma R. Different Levels of CEA, CA153 and CA125 in Milk and Benign and Malignant Nipple Discharge. PLoS One. 2016;11(6):e0157639. doi:10.1371/journal.pone.0157639 [doi]

71. Brunoro GVF, Carvalho PC, Barbosa VC, et al. Differential proteomic comparison of breast cancer secretome using a quantitative paired analysis workflow. BMC Cancer. 2019;19(1):365-019-5547-y. doi:10.1186/s12885-019-5547-y [doi]

72. Loud JT, Thiebaut AC, Abati AD, et al. Ductal lavage in women from BRCA1/2 families: is there a future for ductal lavage in women at increased genetic risk of breast cancer? Cancer Epidemiol Biomarkers Prev. 2009;18(4):1243-1251. doi:10.1158/1055-9965.EPI-08-0795 [doi]

73. Antill YC, Mitchell G, Johnson SA, et al. Gene methylation in breast ductal fluid from BRCA1 and BRCA2 mutation carriers. Cancer Epidemiol Biomarkers Prev. 2010;19(1):265-274. doi:10.1158/10559965.EPI-09-0359 [doi]

74. M.J. F, A. R, W.T. W, et al. Hypermethylated genes as biomarkers of cancer in women with pathologic nipple discharge. Clin Cancer Res. 2009;15(11):3802-3811. doi:10.1158/1078-0432.CCR-08-1981 [doi]

75. Fought AJ, McGathey C, Scholtens DM, et al. Hormonal determinants of nipple aspirate fluid yield among breast cancer cases and screening controls. Cancer Epidemiol Biomarkers Prev. 2013;22(12):22772284. doi:10.1158/1055-9965.EPI-12-0434 [doi]

76. Shao ZM, Nguyen M. Nipple aspiration in diagnosis of breast cancer. Semin Surg Oncol. 2001;20(3):175180. doi:10.1002/ssu.1031 [pii]

77. Wang G, Qin Y, Zhang J, et al. Nipple discharge of CA15-3, CA125, CEA and TSGF as a new biomarker panel for breast cancer. Int J Mol Sci. 2014;15(6):9546-9565. doi:10.3390/ijms15069546 [doi]

78. Zielinski J, Jaworski R, Irga-Jaworska N, Pikula M, Hunerbein M, Jaskiewicz J. Use of fiberoductoscopy for the management of patients with pathological nipple discharge: experience of a single center in Poland. Breast Cancer. 2018;25(6):753-758. doi:10.1007/s12282-018-0883-3 [doi]

79. Zielinski J, Jaworski R, Irga-Jaworska N, Haponiuk I, Jaskiewicz J. The significance of ductoscopy of mammary ducts in the diagnostics of breast neoplasms. Wideochirurgia $\mathrm{i}$ inne Tech maloinwazyjne = Videosurgery other miniinvasive Tech. 2015;10(1):79-86. doi:10.5114/wiitm.2014.46823 [doi]

80. Jacobs VR, Paepke S, Schaaf H, Weber BC, Kiechle-Bahat M. Autofluorescence ductoscopy: a new imaging technique for intraductal breast endoscopy. Clin Breast Cancer. 2007;7(8):619-623. doi:S1526-8209(11)70750-9 [pii]

81. Douplik A, Leong WL, Easson AM, Done S, Netchev G, Wilson BC. Feasibility study of autofluorescence mammary ductoscopy. J Biomed Opt. 2009;14(4):44036. doi:10.1117/1.3210773 [doi] 


\section{Supplementary data}

\section{Study}

YÄ̈Imaz et al. 2017 Zhao et al. 2015

Bahl et al. 2015

Morrogh et al. 2010

Hou et al. 2002

Cabioglu et al. 2003

Baydoun S et al. 2019

Jung H.K. et al. 2019

Li G.Z. et al. 2018

Kan W.M. et al. 2018

Lesetedi C. et al. 2017

Park C.J. et al. 2016

Lorenzon M. et al. 2011

Filipe et al. 2019

Fixed effect model Heterogeneity: $I^{2}=88 \%$.

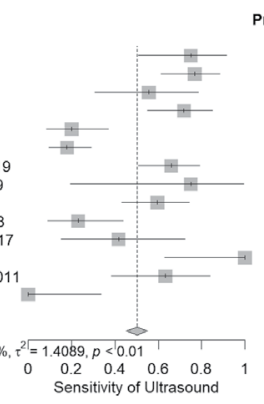

Proportion $\quad 95 \%-\mathrm{Cl}$

Study

$0.75[0.51 ; 0.91]$ $0.77[0.61 ; 0.88]$ $0.56[0.31 ; 0.78$ $0.72[0.55 ; 0.85]$ $0.20[0.08 ; 0.37]$ $0.18[0.10 ; 0.29]$ $0.66[0.51 ; 0.79$ $0.75[0.19 ; 0.99]$ $0.60[0.43 ; 0.74]$ $0.23[0.09 ; 0.44$ $0.42[0.15 ; 0.72]$ $1.00[0.63 ; 1.00]$ $0.63[0.38 ; 0.84]$ $0.00[0.00 ; 0.34]$

$0.50[0.45 ; 0.55]$

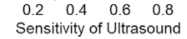

Study

Y $\ddot{A} \pm$ Imaz et al. 2017 Zhao et al. 2015

Bahl et al. 2015

Morrogh et al. 2010

Hou et al. 2002

Cabioglu et al. 2003

Baydoun S et al. 2019

Jung H.K. et al. 2019

Li G.Z. et al. 2018

Kan W.M. et al. 2018

Lesetedi C. et al. 2017

Park C.J. et al. 2016

Filipe et al. 2019

Filipe et

Fixed effect model

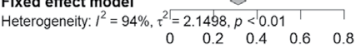

Positive predicted value of Ultrasound

Proportion $\quad 95 \%-\mathrm{Cl}$

0.88 [0.64; 0.99$]$

$0.28[0.20 ; 0.38]$

$0.15[0.07 ; 0.25]$

$0.42[0.30 ; 0.54]$

$0.25[0.11 ; 0.45]$

$0.38[0.21 ; 0.56]$

$0.97[0.84 ; 1.00]$

$0.11[0.02 ; 0.29]$

$0.23[0.15 ; 0.32]$

$0.40[0.16 ; 0.68$

$0.11[0.04 ; 0.25]$

$0.15[0.07,0.28]$

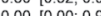

$0.31[0.28 ; 0.35]$

YÄ \pm Imaz et al. 2017

Zhao et al. 2015

Bahl et al. 2015

Morrogh et al. 2010

Hou et al. 2002

Cabioglu et al. 2003

Baydoun S et al. 2019

Jung H.K. et al. 2019

Li G.Z. et al. 2018

Kan W.M. et al. 2018

Park C.J. et al. 2016

Lorenzon M. et al. 2011

Filipe et al. 2019

Fixed effect model

Heterogeneity: $I^{2}=97$

$97 \%, \tau^{2}=$

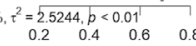

specificity of Ultrasound

Study

YÄıImaz et al. 2017

Zhao et al. 2015

Bahl et al. 2015
Morrogh et al. 2010

Morrogh et al. 2010
Hou et al. 2002

Cabiog. 2002

Cabioglu et al. 2003

Baydoun S et al. 2019

LiG Z et al. 2018

Kan W.M. et al. 2018

Lesetedi C. et al. 2017

ark.J. et al. 2011

Lorenzon M. et al. 2011

Fixed effect model

Heterogeneity: $I^{2}=92 \%, \tau^{2}=1.1761, b<0.01$

Negative predicted value of Ultrasound

0.44 [0.14; 0.79$]$ $0.96[0.91 \cdot 0.98]$ $0.96[0.91 ; 0.98]$ $0.59[0.39 ; 0.78]$ $0.50[0.40 \cdot 0.60]$ $0.64[0.49 ; 0.78]$ $0.64[0.49 ; 0.78]$ $0.87[0.80: 0.92]$ $0.75[0.64 ; 0.84]$ $0.94[0.87 ; 0.97]$ $1.00[0.77 ; 100]$ $0.70[0.47 ; 0.87]$ $0.96[0.92 ; 0.98]$

$0.83[0.81 ; 0.85]$

Proportion $\quad 95 \%-\mathrm{Cl}$
Study

YÄ.Imaz et al. 2017 Zhao et al. 2015 Bahl et al. 2015 Hou et al. 2002 Cabioglu et al. 2003 Baydoun S et al. 2019 Jung H.K. et al. 2019 Li G.Z. et al. 2018 Kan W.M. et al. 2018 Park C.J. et al. 2016 Lorenzon M. et al. 2011 Filipe et al. 2019

Fixed effect model

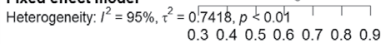

Diagnostic accuracy of Ultrasound Lesetedi C. et al. 2017
$0.73[0.52 ; 0.88]$

$0.39[0.31 ; 0.47$

$0.47[0.36 ; 0.57$

$0.72[0.65 ; 0.79$

$0.47[0.39 ; 0.56$

$0.78[0.67 ; 0.87]$

$0.46[0.31 ; 0.61]$

$0.58[0.52 ; 0.64]$

$0.69[0.59 ; 0.79$

$0.70[0.62 ; 0.77]$

$0.33[0.22 ; 0.45$

$0.74[0.57 ; 0.87$

$0.95[0.91 ; 0.98$

$0.65[0.62 ; 0.67]$

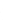

Supplemental Figure 1. Usefulness of Ultrasound as a Modality to Detect Breast Cancer in Patients With Pathologic Nipple Discharge. Sensitivity, Specificity, Positive Predictive Value, Negative Predictive Value, and Diagnostic Accuracy of Ultrasound

Abbreviation: $\mathrm{CI}=$ confidence interval. 


\section{Study}

YÄ \pm Imaz et al. 2017 Zhao et al. 2015 Bahl et al. 2015 Morrogh et al. 2010 Hou et al. 2002 Cabioglu et al. 2003 Baydoun S et al. 2019 Jung H.K. et al. 201 Li G.Z. et al. 2018 Kan W.M. et al. 2018 Lesetedi C. et al. 2017 Park C.J. et al. 2016 Lorenzon M. et al. 201 Filipe et al. 2019

Fixed effect model

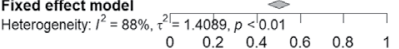

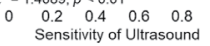

Study

YÄ \pm Imaz et al. 2017 Zhao et al. 2015 Bahl et al. 2015 Morrogh et al. 2010 Hou et al. 2002 Cabioglu et al. 2003 Baydoun S et al. 2019 Jung H.K. et al. 2019 Kan W. M. et al. 2018 Kan W.M. et al. 2018 Lesetedi C. et al. 2017 Lorenzon M et al. 2011 Filipe et al. 2019

Fixed effect model Heterogeneity: $I^{2}=94 \%, \tau^{2}$

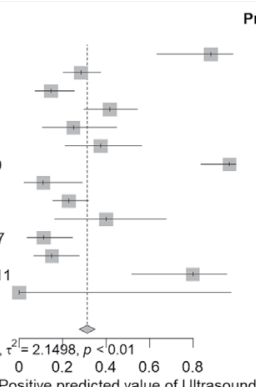

Proportion $\quad 95 \%-\mathrm{Cl}$

0.88 [0 64:0.99] $0.28[0.20 ; 0.38]$ $0.15[0.07 ; 0.25]$ $0.42[0.30 ; 0.54]$ $0.42[0.11,0.45$ $0.38[0.21 ; 0.56]$ $0.38[0.21 ; 0.56]$
$0.97[084 ; 100]$ $0.97[0.84 ; 1.00]$ $0.23[0.15,0.32$ 0.40 [0.16; 0.68 $0.40[0.16,0.68]$ $0.15[0.07: 0.28$ $0.80[0.52 ; 0.96$ $0.80[0.52 ; 0.96$

$0.31[0.28 ; 0.35]$ Positive predicted value of Ultrasound

Proportion $\quad 95 \%-\mathrm{Cl}$

Study

Yä \pm Imaz et al. 2017 Zhao et al. 2015 Bahl et al. 2015 Morrogh et al. 2010 Hou et al. 2002 Cabioglu et al. 2003 Baydoun S et al. 2019 Jung H.K. et al. 2019 Li G.Z. et al. 2018 Kan W.M. et al. 2018 Lesetedi C. et al. 2017 Park C.J. et al. 2016
Lorenzon M. et al. 2011 Filipe et al. 2019

Fixed effect model Heterogeneity: $I^{2}=95 \%, \tau^{2}=0.7418, p<0.04$

$0.30 .40 .5 \quad 0.6 \quad 0.70 .80 .9$ Diagnostic accuracy of Ultrasound

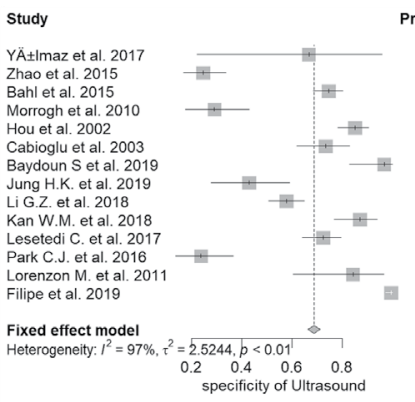

Proportion $\quad 95 \%-\mathrm{Cl}$

$0.67[0.22 ; 0.96]$ $0.25[0.17 ; 0.34]$ $0.75[0.68 ; 0.80]$ $0.29[0.18 ; 0.43]$ $0.85[0.78 ; 0.91]$ $0.73[0.62 ; 0.83]$ $0.97[0.83 ; 1.00]$ $0.43[0.28 ; 0.59]$ $0.58[0.51 ; 0.65]$ $0.87[0.77 ; 0.94]$ $0.72[0.64 ; 0.80]$ $0.24[0.14 ; 0.37]$ $0.99[0.90 ; 0.97]$ $0.69[0.66 ; 0.71]$

\section{Study}

YÄ̇Imaz et al. 2017 Zhao et al. 2015 Morrogh et al. 2010 Hou et al. 2002 Cabioglu et al. 2003 Baydoun S et al. 2019 Jung H.K. et al. 2019 Li G.Z. et al. 2018 Kan W.M. et al. 2018 Lesetedi C. et al. 2017 Lorenzon M. et al. 2011

Fixed effect model

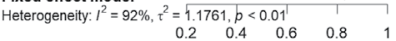
Negative predicted value of Ultrasound
Proportion $\quad 95 \%-\mathrm{Cl}$

$0.44[0.14 ; 0.79]$ $0.73[0.56 ; 0.86]$ $0.96[0.91 ; 0.98]$ $0.59[0.39 ; 0.78]$ $0.81[0.74 ; 0.87]$ $0.50[0.40 ; 0.60]$ $0.64[0.49 ; 0.78]$ $0.95[0.74 ; 1.00]$ $0.87[0.80 ; 0.92]$ $0.75[0.64 ; 0.84]$ $0.94[0.87 ; 0.97]$ $1.00[0.77 ; 1.00]$ $0.70[0.47 ; 0.87]$ $0.83[0.81 ; 0.85]$

Supplemental Figure 2. Usefulness of Mammogram as a Modality to Detect Breast Cancer in Patients With Pathologic Nipple Discharge. Sensitivity, Specificity, Positive Predictive Value, Negative Predictive Value, and Diagnostic Accuracy of Mammogram

Abbreviation: $\mathrm{CI}=$ confidence interval. 
Study

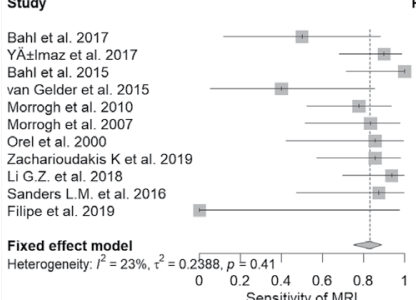

Study

Bahl et al. 2017 YÄtImaz et al. 2017 Bahl et al. 2015 van Gelder et al. 2015 Morrogh et al. 2010 Morrogh et al. 2007 Orel et al. 2000 Zacharioudakis K et al. 2019 Li G.Z. et al. 2018 Sanders L.M. et al. 2016 Filipe et al. 2019

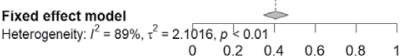

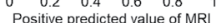
Proportion $\quad 95 \%-\mathrm{Cl}$ $0.50[0.12 ; 0.88]$ $0.90[0.68 ; 0.99]$ $1.00[0.72 ; 1.00]$ $0.40[0.05 ; 0.85$ $0.78[0.52 ; 0.94]$ $0.83[0.52 ; 0.98$ $0.86[0.42 ; 1.00$ $0.86[0.57 ; 0.98]$ $088[0.47,100$ $0.88[0.47 ; 1.00]$

$0.83[0,75,0.09$

$0.83[0.75 ; 0.89]$

Study

Bahl et al. 2017 YAtImaz et al. 2017 van Gelder et al. 2015 Morrogh et al. 2010 Morrogh et al. 2007 Orel et al. 2000 Zacharioudakis $K$ et al. 201 Li G.Z. et al. 2018 Sanders L.M. et al. 2016 Fixed effect model
Heterogeneity: $I^{2}=94 \%, \tau^{2}=2.4064, p<0.01$, $0.3 \quad 0.40 .50 .60 .7$
specificity of MRI

Proportion $\quad 95 \%-\mathrm{Cl}$ $0.76[0.66 ; 0.84]$ $0.68[0.56 ; 0.78$ $0.97[0.92 ; 0.99$ $0.44[0.28 ; 0.62]$ $0.62[0.38 ; 0.82]$ $1.00[0.63,1.00]$ $0.99[0.92 ; 1.00]$ $0.35[0.23,0.49]$ $0.97[0.65 ; 100]$

$0.76[0.72 ; 0.79]$

Study

Proportion $\quad 95 \%-\mathrm{Cl}$ $0.11[0.02 ; 0.29]$ $0.90[0.68 ; 0.99]$ $0.40[0.05,0.85$ $0.41[0.25 ; 0.59$ $0.56[0.31 ; 0.78]$ $1.00[0.54 ; 1.00]$ $0.92[0.64 ; 1.00]$ $0.29[0.17,0.43]$ $0.24[0.10,0.44]$

$0.40[0.34 ; 0.47]$

Bahl et al. 2017 YÃtmaz et al. 2017 Bahl et al. 2015 van Gelder et al. 2015 Morrogh et al. 2010 Morrogh et al. 2007 Orel et al. 2000 Zacharioudakis K et al. 2019 Li G.Z. et al. 2018 Sanders L.M. et al. 201 Filipe et al. 2019 Fixed effect model
Heterogeneity $I^{2}=50 \%, \tau^{2}=0.6171, p=0.04$ $\begin{array}{lllllllllllll}0.3 & 0.4 & 0.5 & 0.6 & 0.7 & 0.8 & 0.9\end{array}$ $0.96[0.89 ; 0.99]$ $0.67[0.22 ; 0.96]$ $0.97[0.92 ; 0.99]$ $0.80[0.56 ; 0.94]$ $0.87[0.60 ; 0.98]$ $0.89[0.52 ; 1.00]$ $0.97[0.90 ; 1.00]$ $0.95[0.76 ; 1.00]$ $0.98[0.90 ; 1.00]$ $0.97[0.85 ; 1.00]$ $0.96[0.93 ; 0.97]$
Study

Bahl
YÄtImaz

Bahl Geld

van Gelder
Morrogh

Morrogh
Morrogh

Orel

Zacharioudakis $\mathrm{K}$

LiG.Z.

Sanders L.M.

Filipe

Fixed effect model

Heterogeneity: $I^{2}=\left.91 \%\right|_{T^{2}}=1.0571, p<0.01 \mid$

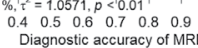

Proportion $\quad 95 \%-\mathrm{Cl}$

$0.74[0.65 ; 0.82]$

$0.85[0.65 ; 0.96]$

$0.71[0.61 ; 0.80]$

$0.94[0.88 ; 0.98$

$0.56[0.41 ; 0.69]$

$0.70[0.51 ; 0.84]$

$0.93[0.68 ; 1.00]$

$0.96[0.90 ; 0.99]$

$0.48[0.36,0.60$

$0.95[0.82 ; 0.99]$

$0.77[0.74 ; 0.80]$

Supplemental Figure 3. Usefulness of MRI as a Modality to Detect Breast Cancer in Patients With Pathologic Nipple Discharge. Sensitivity, Specificity, Positive Predictive Value, Negative Predictive Value, and Diagnostic Accuracy of MRI

Abbreviations: $\mathrm{CI}=$ confidence interval; $\mathrm{MRI}=$ magnetic resonance imaging. 


\section{Study}

Zhao et al. 2015 Dolan et al. 2010 Frank et al. 2010 Morrogh et al. 2010 Kooistra et al. 2009 Coobistra et al. 2009 Simmons et al. 2003 Yamamoto et al. 2003 Hou et al. 2000 Li G.Z. et al. 2018 Kan W.M. et al. 2018 Groves A.M. et al. 1996

Fixed effect model Heterogeneity. $I^{2}=69 \%$,

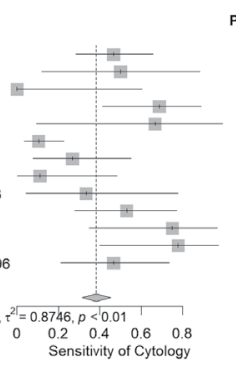

Proportio

$0.47[0.28 ; 0.66]$ $0.50[0.12 ; 0.88]$ $0.09[0.41 ; 0.89]$ $0.69[0.41 ; 0.89]$ $0.67[0.03,0.99]$ $0.10[0.03 ; 0.23]$ $0.11[0.00 ; 0.48$ $0.33[0.04: 0.78$ $0.53[0.28 ; 0.77$ $0.75[0.35 ; 0.97]$ $0.78[0.40 ; 0.97]$ $0.47[0.21 ; 0.73$

$0.38[0.31 ; 0.45]$

Proportion $\quad 95 \%-\mathrm{Cl}$

Study

Zhao et al. 2015 Dolan et al. 2010 Morrogh et al. 2010 Kooistra et al. 2009 Cabioglu et al. 2003 Simmons et al. 2003 Yamamoto et al. 2003 Hou et al. 2000

Li G.Z. et al. 2018
Kan W.M. et al. 2018 Kan W.M. et al. 2018
Groves A.M. et al. 1996

Fixed effect model

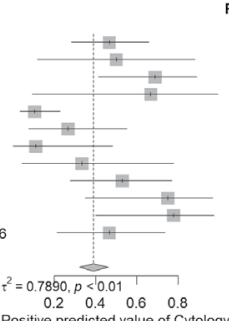

$0.47[0.28 ; 0.66$

$0.50[0.12 ; 0.88$

$0.69[0.41 ; 0.89]$

$0.10[0.03 ; 0.23]$

$0.27[0.08 ; 0.55$

$0.11[0.00 ; 0.48]$

$0.33[0.04 ; 0.78$

$0.53[0.28 ; 0.77]$

$0.75[0.35 ; 0.97]$

$0.78[0.40 ; 0.97]$
$0.47[0.21 ; 0.73]$

$0.39[0.32 ; 0.46]$

Positive predicted value of Cytology

Study

Proportion

Zhao

Dolan

Morrogh

Tekin

Kooistra
Cabioglu

Simmons

Yamamoto

Hou

Kan W.M.

Groves A.M.

Fixed effect mode

Heterogeneity $I^{2}=94 \%, t^{2}=1.4002 p<b .01$

$\%, t^{2}=1.4002, p<0.01$

$\begin{array}{cccccc}0.4 & 0.5 & 0.6 & 0.7 & 0.8 & 0.9 \\ \text { Diagnostic accuracy of Cytology }\end{array}$

Proportion $\quad 95 \%-\mathrm{Cl}$
Study

Zhao et al. 2015

Dolan et al. 2010

Frank et al. 2010
Morrogh et al. 2010

Morrogh et al. 2010
Tekin et al. 2009

Kooistra et al. 2009

Simmons et al. 2003

Yamamoto et al. 2003

Hou et al. 2000

Li G.Z. et al. 2018

Kan W.M. et al. 2018

Groves A.M. et al. 1996

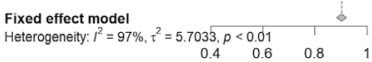

$\begin{array}{ccc}0.4 & 0.6 & 0.8 \\ \text { specificity of Cytology }\end{array}$

Proportion $\quad 95 \%-\mathrm{Cl}$

$1.00[0.94 ; 1.00]$ $1.00[0.95 ; 1.00]$ $0.57[0.34 ; 0.78]$ $0.97[0.83 ; 1.00]$ $0.73[0.64 ; 0.81]$ $0.88[0.80 ; 0.94]$ $0.89[0.77 ; 0.96]$ $0.89[0.81 ; 0.95]$ $0.44[0.28 ; 0.62]$ $1.00[0.97 ; 1.00]$

$0.90[0.88 ; 0.92]$

Study

Zhao et al. 2015 Dolan et al. 2010 Frank et al. 2010 Morrogh et al. 2010 Tekin et al. 2009 Kooistra et al. 2009 Cabioglu et al. 2003 Simmons et al. 2003 Yamamoto et al. 2003 Hou et al. 2000 Li G.Z. et al. 2018 Groves A.M. et al. 199

Groves A.M. et al.

Fixed effect model Heterogeneity: $I^{2}=85 \%, \tau ^ { 2 } = 0 \longdiv { 8 6 2 5 , p < 0 . 0 1 }$

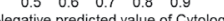

Proportion $\quad 95 \%-\mathrm{Cl}$

$0.80[0.70 ; 0.88]$ $0.96[0.88 ; 0.99]$ $0.98[0.95 ; 1.00]$ $0.71[0.44 ; 0.90]$ $0.97[0.83 ; 1.00]$ $0.66[0.57 ; 0.74]$ $0.89[0.81 ; 0.94]$ $0.76[0.59 ; 0.89]$ $0.92[0.81 ; 0.98]$ $0.91[0.84 ; 0.96]$ $0.89[0.65 ; 0.99$ $0.96[0.93 ; 0.98]$

$0.89[0.87 ; 0.91]$

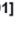

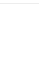

.

Supplemental Figure 4. Usefulness of Cytology as a Modality to Detect Breast Cancer in Patients With Pathologic Nipple Discharge. Sensitivity, Specificity, Positive Predictive Value, Negative Predictive Value, and Diagnostic Accuracy of Cytology

Abbreviation: $\mathrm{CI}=$ confidence interval. 


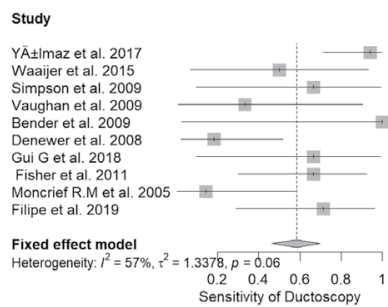

Proportion $\quad 95 \%-\mathrm{Cl}$

\section{Study}

$0.94[0.71 ; 1.00]$
$0.50[0.07 ; 0.93]$

$0.67[0.09 ; 0.99]$

0.33 [0.01; 0.91]

$1.00[0.03 ; 1.00]$

0.18 [0.02; 0.52$]$

$0.67[0.09 ; 0.99]$

$0.67[0.30 ; 0.93]$

$0.71[0.29 ; 0.96]$

$0.58[0.46 ; 0.70]$

YÄIImaz et al. 2017 Simpson et al. 2009 Vaughan et al. 2009 Bender et al. 2009 Denewer et al. 2008 Gui G et al. 2018 Fisher et al. 2011
Moncrief R.M et al. 2005 Filipe et al. 2019 Fixed effect model
Heterogeneity: $I^{2}=93 \%, \tau^{2}=\begin{array}{ccc}4.1912, p<0.01 \\ 0.2 \quad 0.4 & 0.6 & 0.8\end{array}$ specificity of Ductoscopy

Proportion $\quad 95 \%-\mathrm{Cl}$ $0.40[0.05 ; 0.85]$ $0.98[0.89 ; 1.00]$ $0.81[0.64 ; 0.92]$ $1.00[0.96 ; 1.00]$
$1.00[0.96 ; 1.00]$ $1.00[0.96 ; 1.00]$
$0.90[0.77 ; 0.97]$ $0.90[0.77 ; 0.97]$
$1.00[0.88 ; 1.00]$ $0.71[0.61 ; 0.79]$ $0.92[0.81,0.98]$ $0.92[0.89 ; 0.94]$

Study

YÄ \pm Imaz et 2017 Waaijer et al. 2015 Simpson et al. 200 Bender et al. 2009 Denewer et al. 200 Gui $\mathrm{G}$ et al. 2018 Fisher et al. 2011 Moncrief R.M et al. 2005 Filipe et al. 2019

Fixed effect model

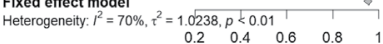
Negative predicted value of Ductoscopy
Proportion $\quad 95 \%-\mathrm{C}$

$0.67[0.09 ; 0.99]$ $0.96[0.86 ; 1.00]$ $0.98[0.92 ; 1.00]$ $1.00[0.96 ; 100$ $0.81[0.67 ; 0.91]$ $0.81[0.67 ; 0.91]$ $0.96[0.90 ; 0.99]$ $0.89[0.77 ; 0.96]$ $0.99[0.95 ; 1.00$

$0.96[0.94 ; 0.97]$

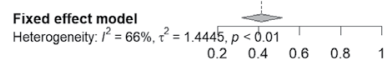
Positive predicted value of Ductoscopy
Study

YÄェImaz et al. 2017 Waaijer et al. 2015 Simpson et al. 2009 Vaughan et al. 2009 Bender et al. 2009 Denewer et al. 2008 Gui G et al. 2018 Fisher et al. 2011 Moncrief R.M et al.

Fixed effect model Heterogeneity: $I^{2}=90 \%, \tau^{2}$

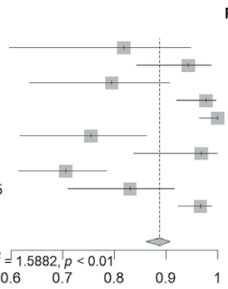

Proportion $\quad 95 \%-\mathrm{Cl}$

$0.82[0.60 ; 0.95]$ $0.94[0.84 ; 0.99]$ $0.79[0.64 ; 0.91]$ $0.98[0.92 ; 1.00]$ $0.75[0.62 ; 0.86]$ $0.97[0.84 ; 1.00]$ $0.71[0.62 ; 0.79]$

$0.89[0.86 ; 0.91]$

Supplemental Figure 5. Usefulness of Ductoscopy as a Modality to Detect Breast Cancer in Patients With Pathologic Nipple Discharge. Sensitivity, Specificity, Positive Predictive Value, Negative Predictive Value, and Diagnostic Accuracy of Ductoscopy

Abbreviation: $\mathrm{CI}=$ confidence interval. 

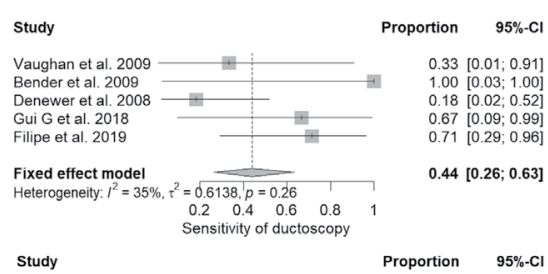

Vaughan et al. 2009

$\begin{array}{lll}\text { Vaughan et al. } 2009 & 0.98[0.92 ; 1.00] \\ \text { Bender et al. } 2009 & 1.00[0.96 ; 1.00]\end{array}$

Denewer et al. $2008+0.81$ [0 67.0.91]

Gui G et al. $2018 \quad-\quad 0.97[0.83 ; 1.00]$

+1
$+\quad 0.99[0.95 ; 1.00]$

Fixed effect model $\quad 0.97[0.94 ; 0.98]$

Heterogeneity: $I^{2}=79 \%, \tau^{2}=1.9222, p<0.01$

Negative predicted value of ductoscopy

Study

Proportion $\quad 95 \%-\mathrm{C}$

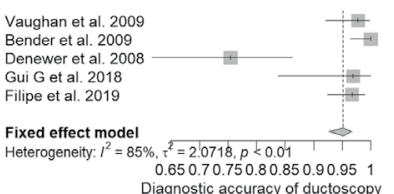

$0.98[0.92 ; 1.00]$ $1.00[0.96 ; 1.00]$

$0.75[0.62 ; 0.86]$

$0.97[0.84 ; 1.00]$

$0.97[0.92 ; 0.99]$

$0.95[0.93 ; 0.97]$

Diagnostic accuracy of ductoscopy

Supplemental Figure 6. Usefulness of Ductoscopy as a Modality to Detect Breast Cancer in Patients With Pathologic Nipple Discharge and Negative Mammogram/Ultrasound Results. Sensitivity, Specificity, Positive Predictive Value, Negative Predictive Value, and Diagnostic Accuracy for Detection of Malignancy by Ductoscopy in Patients With Negative Mammogram/Ultrasound Results

Abbreviation: $\mathrm{CI}=$ confidence interval. 


\section{Study}

Bahl et al. 2017

van Gelder et al. 2015

Morrogh et al. 2007

Zacharioudakis $K$ et al. 2019

Sanders L.M. et al. 2010

Fixed effect model

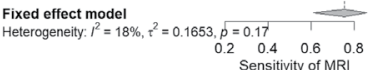

Study

Bahl et al. 2017

van Gelder et al. 2015

Morrogh et al. 2007

Zacharioudakis K et al. 201

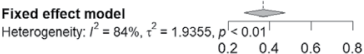

Positive predicted value of MR

Study

Bahl et al. 2017

van Gelder et al. 2015

Morrogh et al. 2007

Zacharioudakis K et al. 2019

Sanders L.M. et al. 201

Fixed effect mode

Heterogeneity: $I^{2}=89 \%, \tau^{2}=\begin{array}{rlll}0.8888, p & p<0.01 \\ 0.6 & 0.7 & 0.8 & 0.9\end{array}$

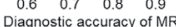

$0.76[0.61 ; 0.86]$

$0.76[0.61 ; 0.86]$

Proportion $\quad 95 \%-\mathrm{C}$

$0.11[0.02 ; 0.29]$ $0.40[0.05 ; 0.85]$ $0.56[0.31 ; 0.78]$ $0.24[0.10 ; 0.44]$

0.37 [0.28; 0.47]

Study

Bahl et al. 2017 van Gelder et al. 2015 Morrogh et al. 2007 Zacharioudakis $K$ et al. 2010

Fixed effect model
Heterogeneity. $I^{2}=93 \%, \tau^{2}=1.9079, p^{1}<0.01$

$\begin{array}{llllll}0.4 & 0.5 & 0.6 & 0.7 & 0.8 & 0.9\end{array}$

Study

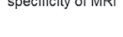

Bahl et al. 2017

van Gelder et al. 2015
Morrogh et al. 2007

Zacharioudakis K et al.

Sanders L.M. et al. 2016

Fixed effect mode

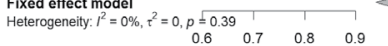

Negative predicted value of MR

Proportion $\quad 95 \%-\mathrm{Cl}$

$0.74[0.65 ; 0.82]$

$0.94[0.88 ; 0.98]$

$0.70[0.51 ; 0.84]$

$0.96[0.90 ; 0.99]$

$0.83[0.79 ; 0.87]$
Proportion $\quad 95 \%-\mathrm{Cl}$

$0.76[0.66 ; 0.84]$ $0.97[0.92 ; 0.99]$ $0.99[0.92 ; 1.00]$ $0.71[0.60 ; 0.81]$

$0.84[0.80 ; 0.88]$

Proportion $\quad 95 \%-\mathrm{Cl}$

$0.96[0.89 ; 0.99]$ $0.97[0.92 ; 0.99]$ $0.87[0.60 ; 0.98]$ $0.97[0.90 ; 1.00]$ $0.98[0.90 ; 1.00]$

$0.97[0.94 ; 0.98]$

Supplemental Figure 7. Usefulness of Ultrasound as a Modality to Detect Breast Cancer in Patients With Pathologic Nipple Discharge. Sensitivity, Specificity, Positive Predictive Value, Negative Predictive Value, and Diagnostic Accuracy for Detection Malignancy of MRI in Patients With Negative Mammogram/Ultrasound Results Abbreviations: $\mathrm{CI}=$ confidence interval; $\mathrm{MRI}=$ magnetic resonance imaging. 
Part 1 - Chapter 2
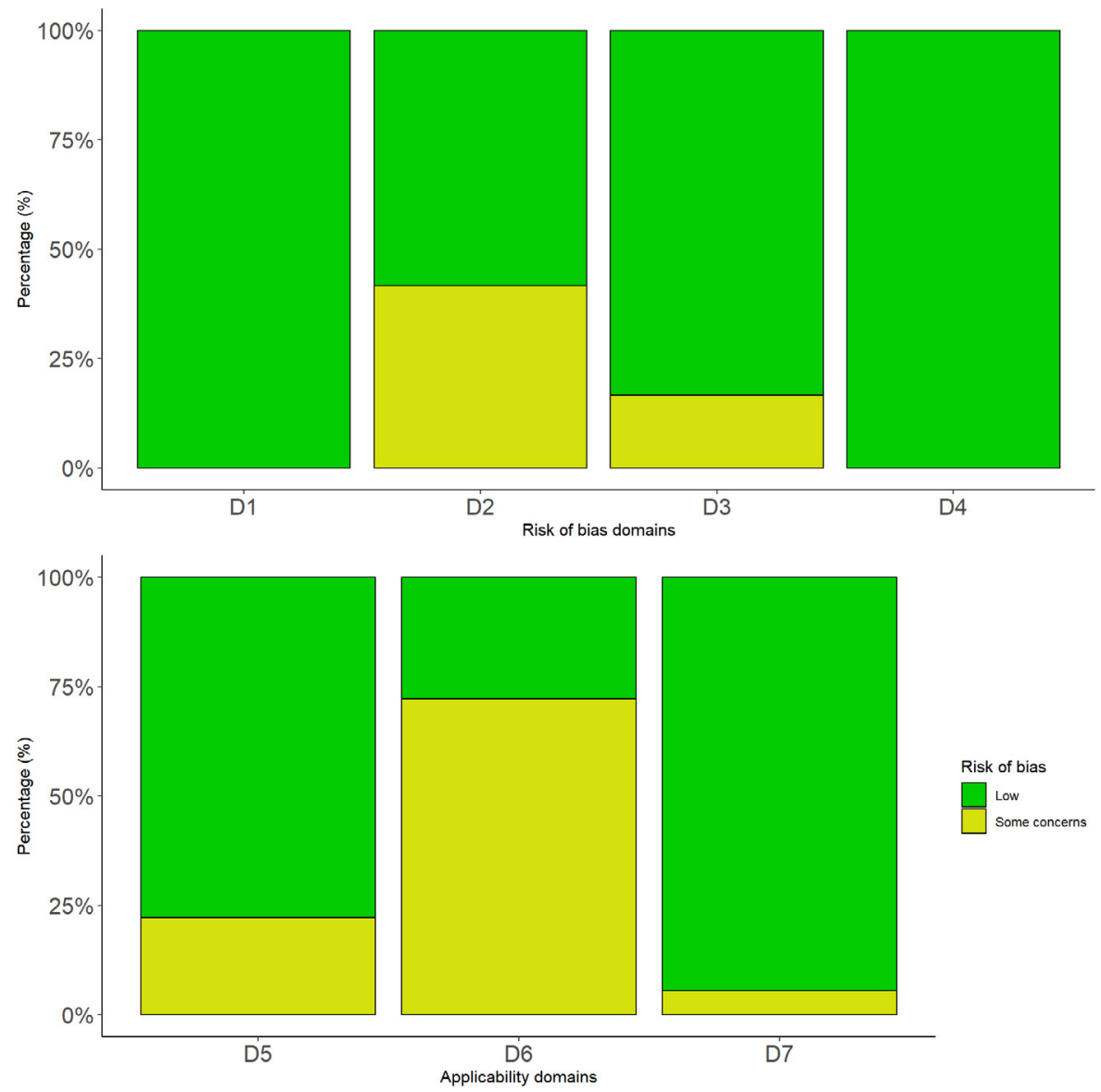

Supplemental Figure 8. Summary of Risks of Bias and Applicability Domains

Abbreviations: D1 = patient selection; D2 = index test; D3 = reference standard; D4 = flow and timing; D5 = patient selection; D6 = index test; D7 = reference standard. 
Supplemental Table 2. Surface Under the Cumulative Ranking Curves (SUCRA) Values of 5 Diagnostic Methods to Detect Malignancy in Patients With Pathologic Nipple Discharge

\begin{tabular}{llllll}
\hline Diagnostic Method & Sensitivity & Specificity & PPV & NPV & DA \\
\hline Ultrasound & 0.5234 & 0.5405 & 0.7066 & 0.9235 & 0.7717 \\
Mammography & 0.9992 & 0.0002 & 0.0192 & 0.8031 & 0.3517 \\
MRI & 0.1659 & 0.8915 & 0.4827 & 0.1674 & 0.9366 \\
Cytology & 0.6994 & 0.2917 & 0.5316 & 0.4099 & 0.0915 \\
Ductoscopy & 0.1121 & 0.7760 & 0.7599 & 0.1962 & 0.3485 \\
\hline
\end{tabular}

Abbreviations: DA = diagnostic accuracy; $\mathrm{MRI}=$ magnetic resonance imaging; $\mathrm{NPV}=$ negative predictive value; $\mathrm{PPV}=$ positive predictive value. 

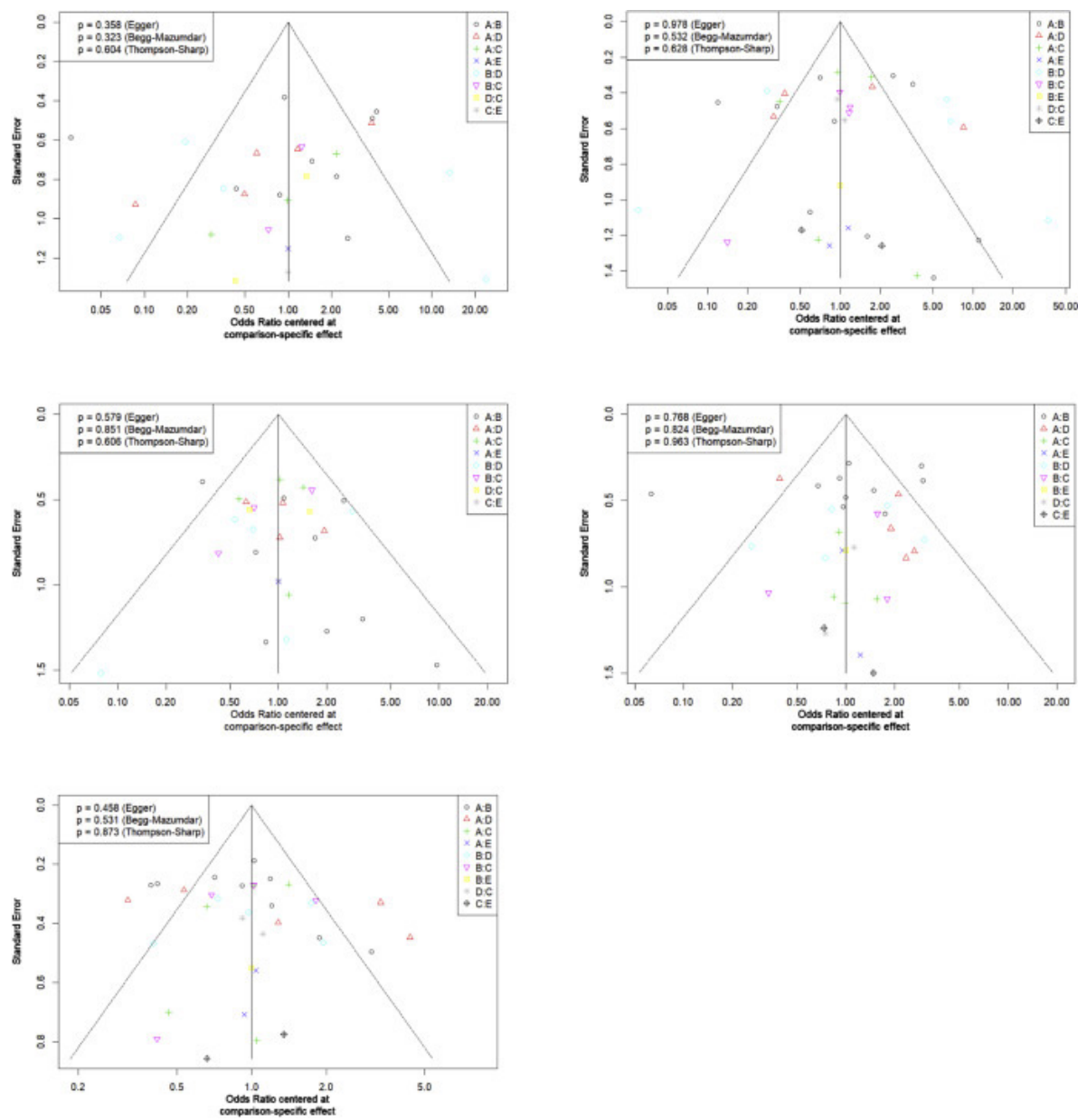

Supplemental Figure 9. Funnel Plots of Potential Publication Bias Regarding Treatment of Pathologic Nipple Discharge. Funnel Plots Showing Potential Publication Bias for Sensitivity (Top Left), Specificity (Top Right), Positive Predictive Value (Middle Left), Negative Predictive Value (Middle Right), and Diagnostic Accuracy (Bottom Left) in a Meta-analysis of Various Approaches to Treat Pathologic Nipple Discharge. Modalities are as Follows: A = Ultrasound; B = Mammogram; C = Magnetic Resonance Imaging; D = Cytology; E = Ductoscopy. 

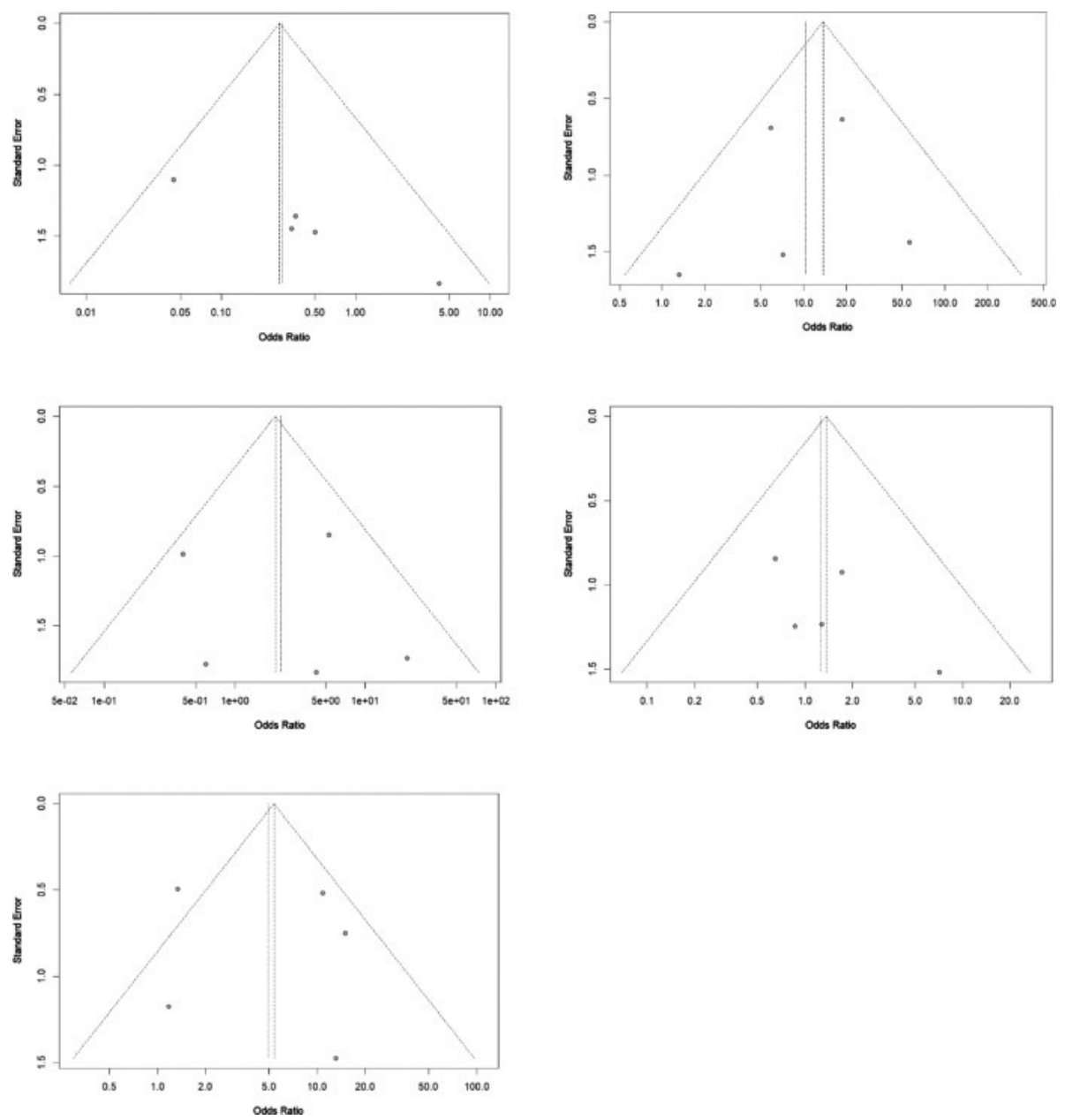

Supplemental Table 1. Pairwise Meta-analysis and Network Meta-analysis Comparing Diagnostic Accuracy in 5 Different Diagnostic Methods 


\section{Chapter 3}

Interventional ductoscopy as an alternative for major duct excision or microdochectomy in women suffering pathologic nipple discharge: a single-center experience

M.D. Filipe

L. Waaijer

C.C. van der Pol

P.J. van Diest

A.J. Witkamp 


\section{Abstract}

\section{Introduction}

Pathologic nipple discharge (PND) is, after palpable lumps and pain, the most common breast-related reason for referral to the breast surgeon and is associated with breast cancer. However, with negative mammography and ultrasound, the chance of PND being caused by malignancy is between $5 \%$ and $8 \%$. Nevertheless, most patients with PND still undergo surgery in order to rule out malignancy. Ductoscopy is a minimally invasive endoscopic technique that enables direct intraductal visualization. The aim of this study was to evaluate (interventional) ductoscopy as an alternative to surgery in patients with negative conventional imaging.

\section{Materials and Methods}

All patients with PND referred between 2010 and 2017 to our hospital for ductoscopy were retrospectively analyzed. Ductoscopy procedures were performed under local anesthesia in the outpatient clinic. The follow-up period was at least 3 months, and the primary outcome was the number of prevented surgical procedures. Furthermore, we evaluated possible complications after ductoscopy (infection and pain).

\section{Results}

A total of 215 consecutive patients undergoing ductoscopy were analyzed. In 151 (70.2\%) patients, ductoscopy was successful. In 102 procedures, an underlying cause for PND was visualized, of which 34 patients could be histologically proven and 82 patients treated. Sixty of the 215 patients were eventually operated, 8 owing to suspicious findings during ductoscopy, 42 owing to persistent PND, and 10 because of recurrent PND. In 7 patients, a malignancy was found ( 5 of them classified as suspicious at dusctoscopy). No serious side effects were seen.

\section{Conclusion}

Ductoscopy can be safely used as an alternative for surgery in the workup for PND. 


\section{Introduction}

Pathologic nipple discharge (PND) is the third most common breast-related complaint, after pain and palpable lumps. ${ }^{1}$ PND is defined as unilateral, spontaneous, and bloody or serous discharge, usually arising from a single duct orifice of the nipple. PND is regarded as a possible sign of breast cancer, and it accounts for 3\% to 5\% of surgical breast clinic referrals. ${ }^{2-5}$ However, when ultrasound and mammography are negative, the risk of malignancy is still around $5 \%$ to $8 \%{ }^{6,7}$ The most common causes of PND are benign: ductal ectasia and intraductal papillomas. ${ }^{8,9}$

Mammography and breast ultrasound are important tools for the detection of breast cancer. However, in the case of PND as the only complaint, they both have limited sensitivity (15\% and 56\% for mammography and ultrasound, respectively). ${ }^{10}$ Magnetic resonance imaging (MRI) has shown to be a sensitive tool for the detection of malignancy, but specificity is low. However, detection of small lesions and differentiating them benign from malignant masses remains difficult in using MRI as a diagnostic tool. ${ }^{11,12}$ Therefore, the value of MRI is limited in patients with PND, and core needle biopsy or surgical excision is still indicated when MRI shows a suspicious lesion. ${ }^{13,14}$

Because PND is regarded as a possible sign of breast cancer, and standard radiologic imaging often fails to reveal the underlying cause, most women suffering PND undergo surgical procedures, such as microdochectomy or major duct excision, to rule out malignancy. ${ }^{8,10,11}$ These surgical procedures are performed under general anesthesia and are associated with scarring, which may result in breastfeeding difficulties in fertile women. ${ }^{15}$ Furthermore, malignancy is found in only $5 \%$ to $8 \%$ of patients. ${ }^{5-7}$ This means that around $90 \%$ to $95 \%$ of these surgical procedures are performed for benign lesions.

Ductoscopy is a minimally invasive micro-endoscopic technique, which makes real-time visualization of the milk ducts of the breast possible. The procedure can be performed under local anesthesia at the outpatient clinic and is currently used as a diagnostic tool in the workup of women suffering from PND without suspicious radiologic findings. ${ }^{16-22}$ Previous studies showed the success of ductoscopy in finding the intraductal lesion causing PND before or during duct excision. ${ }^{23-27}$ In recent years, a biopsy tool was introduced that can be used through the working channel of the ductoscope, enabling interventional ductoscopy to not only visualize but also remove the lesion underlying PND in a single procedure under local anesthesia. ${ }^{27,28}$ Interventional ductoscopy has already been described in a few studies as a safe alternative for classic open surgery in patients with $\mathrm{PND},{ }^{28,29}$ but wider implementation requires further validation studies.

In the present study, we describe the experience with interventional ductoscopy as an alternative to surgery in women with PND in a single national referral center in The Netherlands between 2010 and 2017. 


\section{Materials and Methods}

\section{Patient Selection}

This retrospective observational consecutive cohort study included women who presented with unilateral PND between 2010 and 2017 in the University Medical Centre Utrecht (UMCU) in the Netherlands. This included a smaller cohort of 82 patients on whom we reported before. ${ }^{28}$ Inclusion criteria were patients with spontaneous PND lasting over at least 3 months. Only the first ductoscopy was included for analysis. Exclusion criteria for analysis were the possible subsequent ductoscopy procedures, radiologic and/ or pathologic suspicion of malignancy, or a follow-up period of less than 3 months.

Diagnostic workup of every patient was paramount for this study. Before ductoscopy, patients underwent imaging consisting of ultrasound and/or mammography. Patients received an additional MRI and/or core needle biopsy prior to ductoscopy when there was palpable mass and/or a Breast Imaging Reporting and Data System (BI-RADS) IV. When these additional tests were negative for malignancy (thereby downgrading the initial BI-RADS classification), these patients were eligible for ductoscopy. Either the UMCU (tertiary referring hospital) or the referring hospital performed the diagnostic workup. The ethical committee of the UMCU approved this study and decided that informed consent was not required as data were processed anonymously.

\section{Cannulation}

The surgeon performed the ductoscopy in the outpatient setting as described previously. ${ }^{28}$ First, the surgeon identified the affected duct by pressing the nipple. Patients that did not have spontaneous PND during ductoscopy received oxytocin nose spray 30 minutes prior to the procedure in order to better locate the affected duct. The next step was to disinfect the areola and the nipple with $70 \%$ ethanol. Lidocaine $1 \%$ was used for local anesthesia of the nipple. Salivary duct probe (size 0000 to 1; Karl Storz, Tuttlingen, Germany) and an obturator (Polydiagnost, Pfaffenhofen, Germany) widened the lactiferous duct of the nipple. Stretching the nipple outwards was important in order to straighten the milk ducts to facilitate cannulation. Next, a port through which the ductoscope was introduced (SoLex nipple expander; Polydiagnost) was placed into the affected duct.

\section{Ductoscopy}

Ductoscopy was performed using a 6000-pixel 0.55-mm optic (LaDuScope T-flex; Polydiagnost) and a Polyshaft (1.15-mm outer diameter, PD-DS-1015; Polydiagnost). The Polyshaft system has 3 channels: 1 for the endoscope, 1 for saline irrigation or additional intraductal anesthetic infusion, and 1 for the endobasket. The ductoscope has a working length of $80 \mathrm{~mm}$, a $0^{\circ}$ angle direct view and a field vision of $70^{\circ}$, and is gas-sterilized. 
Visualization of the ductal tree started in the lactiferous duct. The surgeon explored the major ducts in an orderly fashion until the ducts became too narrow to pass. Continuous saline irrigation into the ductal tree through the polyshaft is essential to keep the ducts from collapsing. When necessary, additional intraductal anesthesia (bupivacaine) was administered. One of 2 specialized surgeons with an experience of over 10 ductoscopy procedures performed the procedure. Ductoscopy was regarded as successful when a thorough examination of the afflicted ductal tree was possible.

Possible findings during the assessment of the ductal tree are normal duct morphology, polypoid lesions, ductitis, epithelial lesions/damage, etc. When possible, the endobasket facilitated the extraction of the lesion and subsequent histologic examination for diagnosis. When there was no visible intraductal lesion left after ductoscopy, it was defined as complete removal. In case of a visible residual lesion after extraction that was not possible to extract, it was defined as a partial extraction. Reasons to abort the ductoscopy were intolerable pain or perforation of the duct wall hampering further inspection of the underlying ducts.

\section{Complications}

Two weeks after ductoscopy, the attending physician contacted patients in order to gather information about pain, infections, or any other possible side effect or complication. A scale of 1 to 3 was used to code the pain (no pain, mild pain, or severe pain).

\section{Follow-up}

Patients were at least followed after 2 weeks and 3 months post ductoscopy. Depending on the outcome of the ductoscopy (suspicious findings, persistent PND, and/or patient preference), they were scheduled for surgery or follow-up.

\section{Statistics}

First, normality was determined using Kurtosis, in which z-values between -3 and 3 were considered as normally distributed data. Normally distributed continuous data was described by means and standard deviations. In non-continuous not normally distributed data, median and interquartile range were used to describe the data. For categorical values, the $\chi 2$ test or the Fisher exact test (if the expected value in each cell was less than 5) was used to assess differences between groups. Sensitivity and specificity with $95 \%$ confidence intervals (CIs) were also assessed. P values below .05 were considered to be significant. Statistical analysis of the database was performed using SPSS v.23 (IBM, Armonk, NY). 


\section{Results}

The flowchart in Figure 1 depicts the patients selected for analysis. Between 2010 and 2017, 244 patients with PND underwent a ductoscopy. Seventeen of these patients underwent multiple ductoscopy procedures, resulting in 261 procedures. This analysis only included the first ductoscopy. One male patient was excluded for further analysis. Twenty-eight patients were lost to follow-up, leaving 215 patients suitable for analysis.

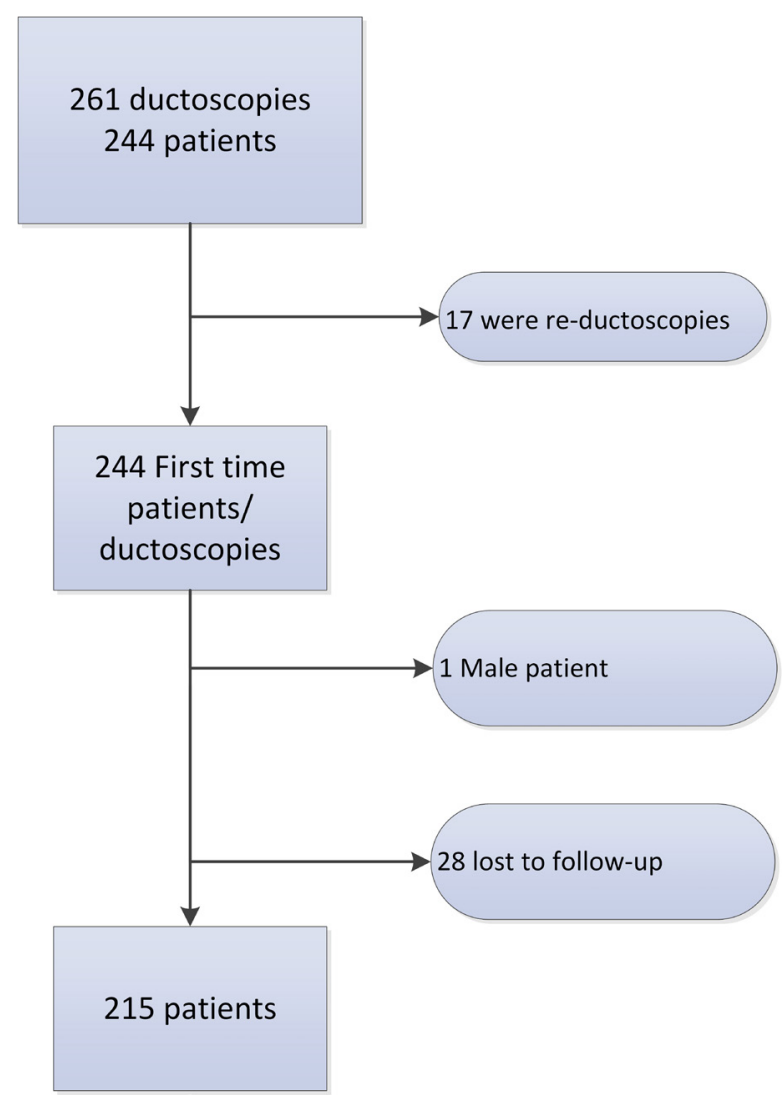

Figure 1. Flowchart Showing all Ductoscopies Performed in Patients With Pathologic Nipple Discharge and Consequent Selection for Analysis

Table 1 shows the baseline characteristics of the 215 patients suitable for analysis. The mean age was 49 years (range, 20-81 years), with a mean follow-up of 14.5 months (range, 3-44 months). Six (2.8\%) patients had palpable abnormalities at the time of ductoscopy. 
Table 1. Clinical Data of 215 Patients With PND Undergoing Ductoscopy

\begin{tabular}{|c|c|c|}
\hline & & $\mathrm{N}=215$ patients \\
\hline Age years (SD) & & $49.2(13.6)$ \\
\hline FU months (SD) & & $14.1(11.4)$ \\
\hline \multirow[t]{3}{*}{ Affected breast N (\%) } & Left & $106(49.3 \%)$ \\
\hline & Right & $100(46.5 \%)$ \\
\hline & Both & $9(4.2 \%)$ \\
\hline \multirow[t]{6}{*}{ Previous pathology N (\%) } & Papilloma & $32(54.2 \%)$ \\
\hline & Benign/normal & $22(37.3 \%)$ \\
\hline & Atypical cells & $1(1.7 \%)$ \\
\hline & Infectious cells & $2(3.4 \%)$ \\
\hline & Other & $2(3.4 \%)$ \\
\hline & Not performed & 156 \\
\hline \multirow[t]{6}{*}{ Cytology PND N (\%) } & Normal/benign & $53(51.5 \%)$ \\
\hline & Papilloma & $30(29.1 \%)$ \\
\hline & Atypical cells & $11(10.7 \%)$ \\
\hline & Infectious cells & $7(6.8 \%)$ \\
\hline & Other & $2(1.9 \%)$ \\
\hline & Not performed & 122 \\
\hline \multirow[t]{6}{*}{ US BI-RADS classification N (\%) } & BI-RADS I & $127(61.7 \%)$ \\
\hline & BI-RADS II & $73(35.4 \%)$ \\
\hline & BI-RADS III & $4(1.9 \%)$ \\
\hline & BI-RADS IVa & $1(0.5 \%)$ \\
\hline & Other & $1(0.5 \%)$ \\
\hline & Not performed & 9 \\
\hline \multirow[t]{6}{*}{ Mammography BI-RADS classification N (\%) } & BI-RADS I & $166(81.8 \%)$ \\
\hline & BI-RADS II & $33(16.3 \%)$ \\
\hline & BI-RADS III & $1(0.5 \%)$ \\
\hline & BI-RADS IVa & $2(1 \%)$ \\
\hline & Other & $1(0.5 \%)$ \\
\hline & Not performed & 12 \\
\hline \multirow[t]{5}{*}{ MRI BI-RADS classification N (\%) } & BI-RADS I & $21(56.8 \%)$ \\
\hline & BI-RADS II & $14(37.8 \%)$ \\
\hline & BI-RADS III & $1(2.7 \%)$ \\
\hline & BI-RADS IVa & $1(2.7 \%)$ \\
\hline & Not performed & 178 \\
\hline
\end{tabular}

Abbreviations: BI-RADS = Breast Imaging Reporting and Data System; MRI = magnetic resonance imaging; $\mathrm{PND}=$ pathologic nipple discharge; $\mathrm{SD}=$ standard deviation 
Sixty of the 215 patients had had a biopsy prior to the ductoscopy. Histology revealed that $32(54.2 \%)$ patients had a papilloma, and in 24 (40.7\%) patients, normal or benign tissue was diagnosed. Atypical morphology and infection were diagnosed in $1(1.7 \%)$ and $2(3.4 \%)$ patients, respectively.

Cytology of the nipple discharge prior to ductoscopy was performed in 103 patients. In $53(51.5 \%)$ patients, cytology showed no abnormalities or was benign. Papilloma was cytologically diagnosed in $30(29.1 \%)$ patients. Eleven $(10.7 \%)$ patients showed atypical cells during cytologic examination. Inflammatory cells were seen in $7(6.8 \%)$ patients with examined nipple discharge. In 2 (1.9\%) patients, cytologic analysis was not conclusive with no signs of malignancy. Initial BI-RADS classification for ultrasound and mammography can be seen in Table 1 .

\section{Ductoscopy}

Figure 2 and Table 2 show the results of the ductoscopy procedures. In 151 (70.2\%) patients, it was possible to visualize the ductal tree, 149 with full ductoscopy report. Sixty-eight $(45.6 \%)$ of these 149 successful ductoscopies showed a polypoid lesion, 49 (32.9\%) displayed no abnormalities, and 19 (12.7\%) depicted flat epithelial lesions. Eight $(5.4 \%)$ ductoscopies showed suspicious lesions; there was $1(0.7 \%)$ duct ectasia as single finding and the remaining $4(2.7 \%)$ were not otherwise specified than not suspicious for malignancy. Of the 49 attempted basket extractions, 34 biopsies were suitable for histopathologic examination. Thirty-three of these lesions turned out to be papillomas (without atypia) according to pathologic analysis, and 1 was found to be normal ductal tissue. Additionally, none of the 34 patients with basket extraction biopsies suitable for histopathologic analysis developed breast cancer in the future. 


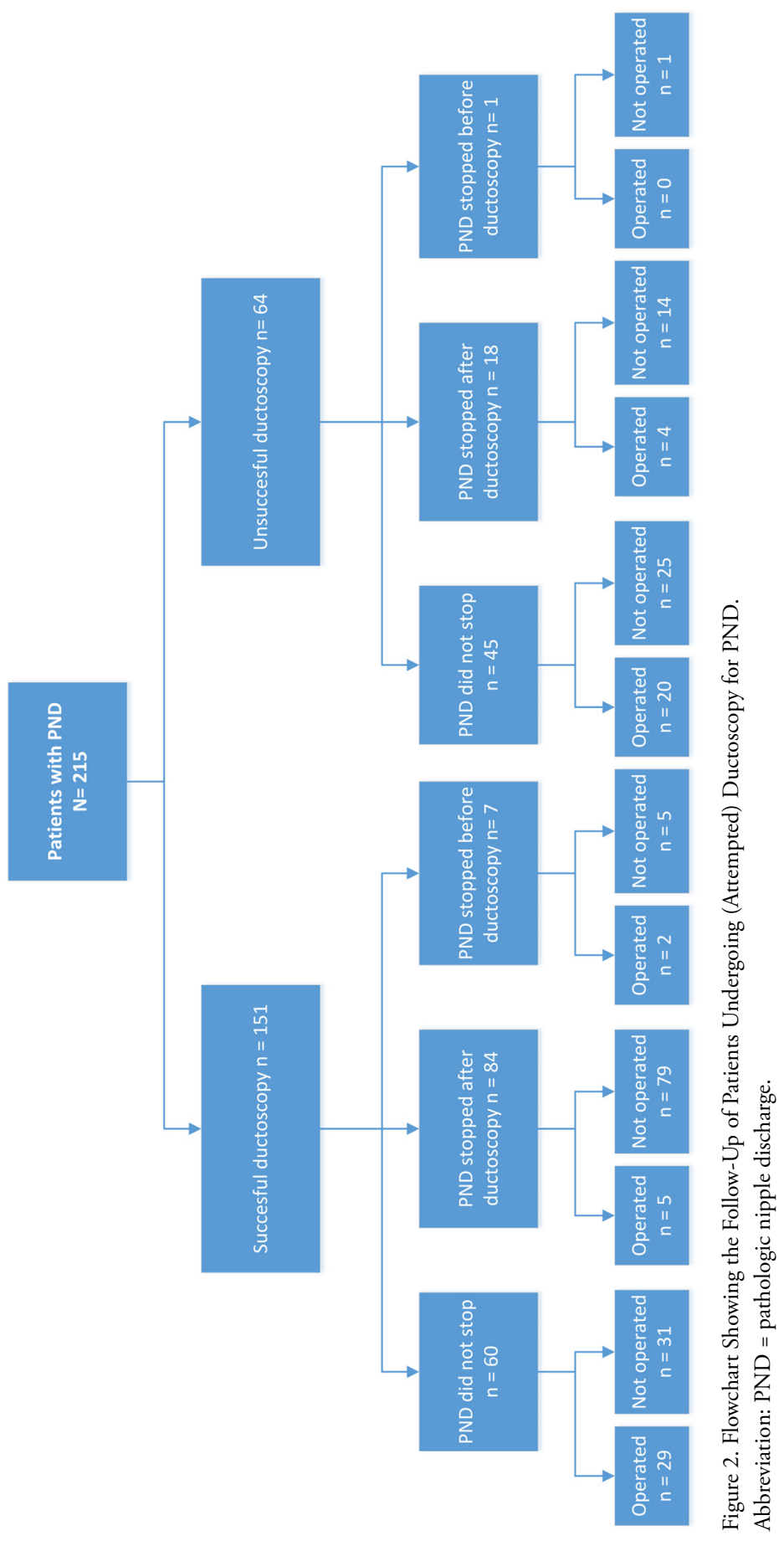


Table 2Findings At and After Ductoscopy of 215 Patients With PND

\begin{tabular}{|c|c|c|c|c|}
\hline & & $\begin{array}{l}\text { Successful } \\
\text { ductoscopy } \\
N=151\end{array}$ & $\begin{array}{l}\text { Unsuccessful } \\
\text { ductoscopy } \\
\mathrm{N}=64\end{array}$ & P value \\
\hline \multirow{6}{*}{$\begin{array}{l}\text { Ductoscopic diagnosis } \\
\text { N (\%) }\end{array}$} & Polypoid lesion & $68(45.6 \%)$ & NA & \\
\hline & Epithelial Lesion & $19(12.7 \%)$ & NA & \\
\hline & Dilated ducts & $1(0.7 \%)$ & NA & \\
\hline & Normal & $49(32.9 \%)$ & NA & \\
\hline & Suspicious & $8(5.4 \%)$ & NA & \\
\hline & Other & $4(2.7 \%)$ & NA & \\
\hline \multirow{2}{*}{$\begin{array}{l}\text { Lesion (partially) } \\
\text { removed N (\%) }\end{array}$} & No & $84(63.2 \%)$ & NA & \\
\hline & Yes & $49(36.8 \%)$ & NA & \\
\hline \multirow{2}{*}{$\begin{array}{l}\text { Pathological analysis } \\
\text { of (partially) removed } \\
\text { lesion (\%) }\end{array}$} & Papilloma & $33(97.1 \%)$ & NA & \\
\hline & Normal tissue & $1(2.9 \%)$ & NA & \\
\hline \multirow{3}{*}{$\begin{array}{l}\text { Pain after ductoscopy } \\
\text { N (\%) }\end{array}$} & No & $131(86.8 \%)$ & $52(81.3 \%)$ & $0.182^{*}$ \\
\hline & Little & $15(9.9 \%)$ & $6(9.4 \%)$ & \\
\hline & High & $5(3.3 \%)$ & $6(9.4 \%)$ & \\
\hline \multirow{2}{*}{$\begin{array}{l}\text { Infection after } \\
\text { ductoscopy N (\%) }\end{array}$} & No & $148(98 \%)$ & $62(96.9 \%)$ & $0.635 \dagger$ \\
\hline & Yes & $3(2 \%)$ & $2(3.1 \%)$ & \\
\hline \multirow{3}{*}{$\begin{array}{l}\text { PND stopped after } \\
\text { ductoscopy N (\%) }\end{array}$} & No & $60(39.7 \%)$ & $45(70.3 \%)$ & $<0.001 \dagger$ \\
\hline & Yes, spontaneous before ductoscopy & $7(4.6 \%)$ & $1(1.6 \%)$ & \\
\hline & Yes, after ductoscopy & $84(55.6 \%)$ & $18(28.1 \%)$ & \\
\hline \multirow{2}{*}{$\begin{array}{l}\text { Operated after } \\
\text { ductoscopy N (\%) }\end{array}$} & Not operated & $115(76.2 \%)$ & $40(62.5 \%)$ & $0.047 \dagger$ \\
\hline & operated & $36(23.8 \%)$ & $24(37.5 \%)$ & \\
\hline \multirow{4}{*}{$\begin{array}{l}\text { Pathologic diagnosis } \\
\text { after operation N (\%) }\end{array}$} & Benign & $7(21.2 \%)$ & $7(31.8 \%)$ & $0.598 \dagger$ \\
\hline & (pre)malignancy & $7(21.2 \%)$ & $2(9.1 \%)$ & \\
\hline & Papilloma & $17(51.5 \%)$ & $11(50 \%)$ & \\
\hline & Widened ducts & $2(6.1 \%)$ & $2(9.1 \%)$ & \\
\hline \multirow{5}{*}{$\begin{array}{l}\text { Decision whether } \\
\text { to operate after } \\
\text { ductoscopy N (\%) }\end{array}$} & Persistent symptoms & $21(13.9 \%)$ & $21(32.8 \%)$ & $<0.001 \dagger$ \\
\hline & Recurrent symptoms & $7(4.7 \%)$ & $3(4.7 \%)$ & \\
\hline & Findings ductoscopy & $8(5.3 \%)$ & $0(0 \%)$ & \\
\hline & No operation, patient is reassured & $46(30.4 \%)$ & $27(42.2 \%)$ & \\
\hline & No operations, no complaints & $69(45.7 \%)$ & $13(20.3 \%)$ & \\
\hline
\end{tabular}

Abbreviations: $\mathrm{N}=$ number; $\mathrm{NA}=$ not applicable; $\mathrm{PND}=$ pathologic nipple discharge

* P-values determined using Chi-Square test

$\dagger$ P-values determined using Fisher's Exact test 
Ductoscopy did not succeed in 64 patients, 62 with an available report. The ductoscopy failed because of perforation through the ductal wall, making further inspection impossible in $28(45.2 \%)$ of the 62 patients. Sixteen (25.8\%) patients underwent attempted ductoscopy despite a relative contraindication (retracted nipple or previous operation on the mammilla). Too narrow ducts impeded proper visualization of the ductal tree in $14(22.6 \%)$ patients. The ductoscopy failed in the remaining 4 (6.4\%), owing to total occlusion of a milk duct because of an obstructive lesion.

\section{Follow-up}

Follow-up data were available for all of the 151 successful ductoscopy procedures. After successful ductoscopy, PND stopped in 84 (55.6\%) patients. In 7 (4.6\%) patients, the PND had already spontaneously stopped at the time of the ductoscopy. The PND did not stop in $60(39.7 \%)$ of the 151 successful ductoscopy procedures.

Follow-up PND data were available for all of the 64 patients in whom ductoscopy was unsuccessful. Of these patients, $45(62.7 \%)$ still complained of PND. The PND stopped after unsuccessful ductoscopy in $18(28.1 \%)$ patients. In 1 (1.6\%) patient, the PND spontaneously resolved before attempted ductoscopy. PND stopped significantly more often in patients with a successful ductoscopy $(\mathrm{P}<.001)$.

\section{Surgery After Ductoscopy}

Figure 3 and Table 2 show the decisions to operate and the outcome respectively. Sixty (27.8\%) of 215 patients were operated on after ductoscopy. Forty-two (70.0\%) of these 60 patients underwent an operation because of persistent symptoms within a few weeks after ductoscopy. Eight (13.3\%) patients had suspicious findings during ductoscopy, and $10(16.7 \%)$ patients underwent an operation owing to recurrent symptoms during followup. One $(1.7 \%)$ patient had a breast amputation after 2 irradical duct excisions showing malignancy. Eight (13.3\%) patients underwent a local excision, $24(40.0 \%)$ a central duct excision, and $27(45.0 \%)$ a microdochectomy. From 55 patients, pathology reports were available for evaluation: $4(7.3 \%)$ patients had duct ectasia as the only finding, 7 (12.7\%) showed ductal carcinoma in situ (DCIS), 44 (73\%) had a benign lesion, of which $28(51 \%)$ were a papilloma without atypia. Patients with a successful ductoscopy were significantly less likely to be operated $(\mathrm{P}=.047)$. 
Part 1 - Chapter 3

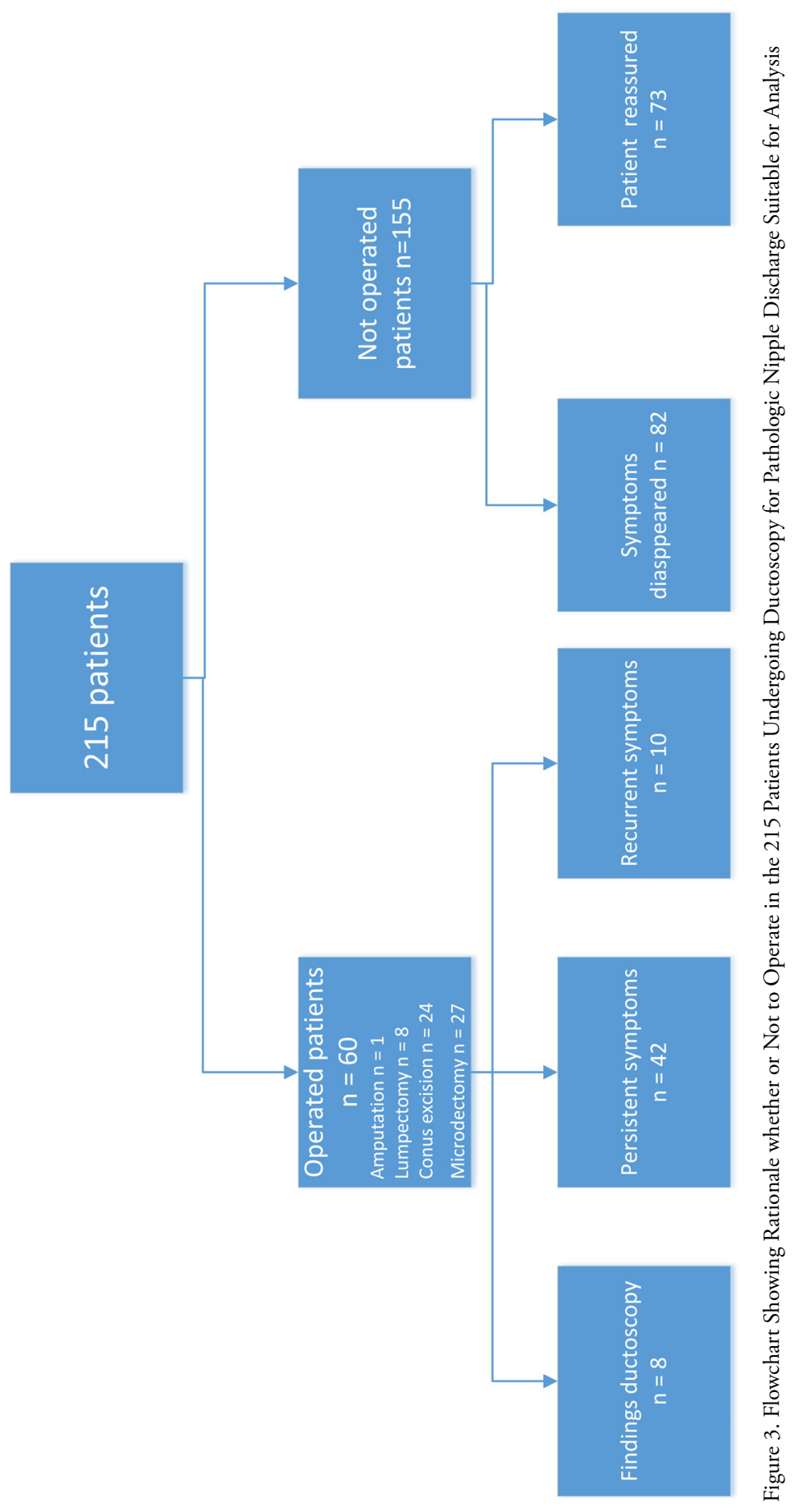


The reasons not to operate were recorded in all of the 155 patients that were not operated. In $82(52.9 \%)$ of them, the PND symptoms resolved spontaneously after the attempted ductoscopy. The remaining $73(47.1 \%)$ patients were sufficiently reassured that (pre) malignancy was ruled out to abandon surgery despite having mild complaints of PND. They were all offered yearly follow-up with mammography and ultrasound. None of them developed (pre)malignancy during follow-up (mean, 14.4 months; range, 3-44.6 months).

\section{Malignancy in Patients with PND}

Eight (3.7\%) patients of the 215 with PND and no suspicious radiologic or pathologic findings were diagnosed with (pre)malignancy after surgery (Table 3). Ductoscopy was unsuccessful in $2(25.0 \%)$ of these patients. These patients were operated owing to persistent serious symptoms. Another $3(37.5 \%)$ patients underwent an operation owing to suspicious findings during ductoscopy; pathologic diagnosis revealed DCIS in 2 patients and lobular carcinoma in situ (LCIS) in 1. In 1 (12.5\%) patient, ductoscopy images suggested ductitis. Intraductal biopsy possible was not possible, so the patient was operated on when PND persisted after ductoscopy. Pathologic diagnosis after surgery revealed DCIS. One (12.5\%) patient did not have suspicious intraductal lesions during ductoscopy, but a suspicious lesion was seen at the duct orifice on the outside of the nipple. This lesion was biopsied, and pathology revealed DCIS. Two (25.0\%) patients with DCIS did not have any suspicious findings during ductoscopy. One of these patients underwent duct excision owing to persistent symptoms, and pathology did not reveal (pre)malignancy. However, DCIS was diagnosed in the breast reduction specimen performed for cosmetic reasons 45 months after initial ductoscopy. The other patient without suspicious findings underwent surgery owing to persistent PND. 


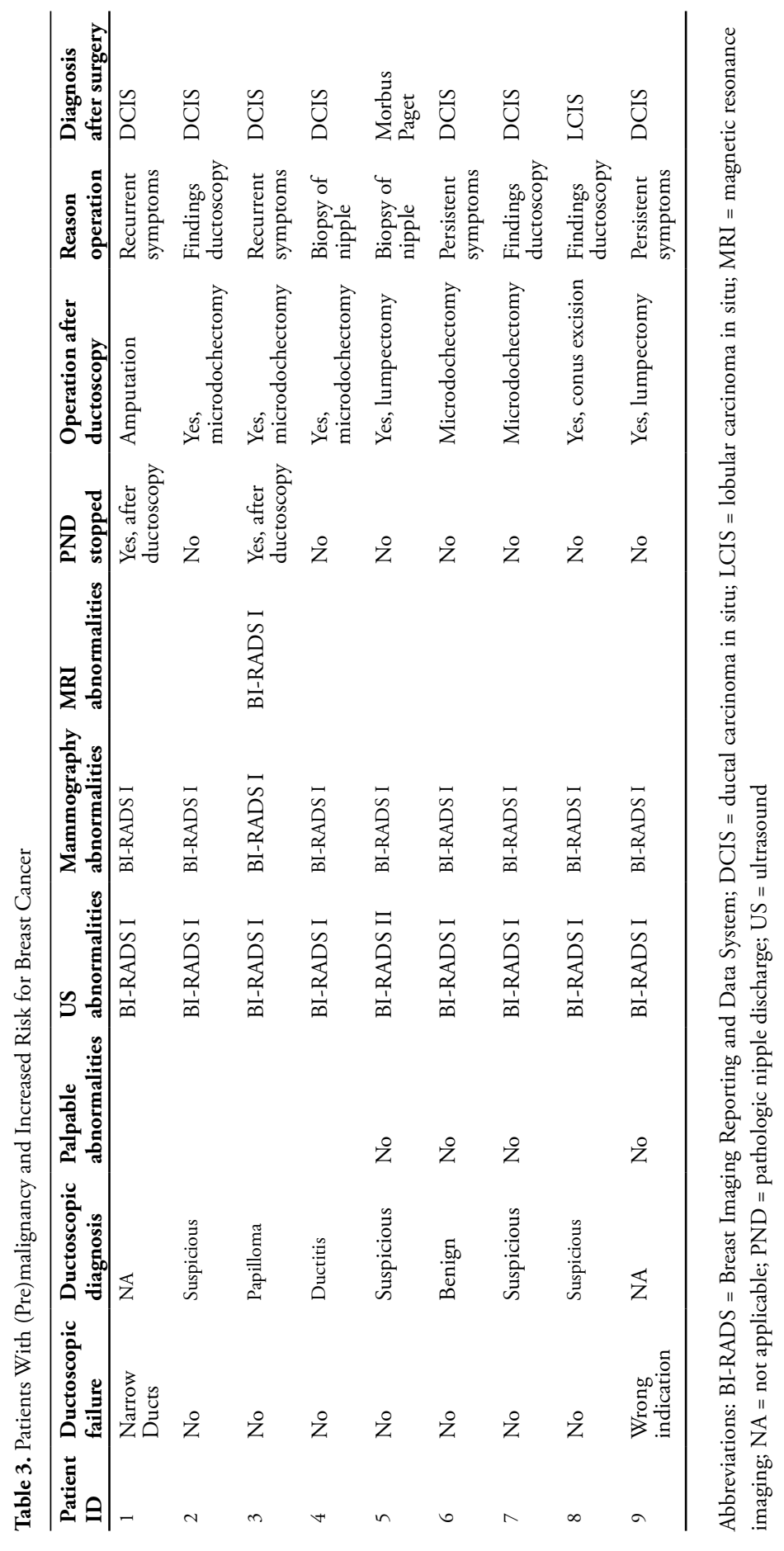




\section{Sensitivity and Specificity}

Summary of the diagnostic value of ductoscopy is summarized in Table 4. Eight $(5.3 \%)$ of the 151 successful ductoscopies revealed suspicious findings leading to an operation, revealing DCIS in 4 patients and LCIS in 1 patient, with the other 3 patients showing benign lesions. Additionally, 4 of the 6 patients diagnosed with DCIS showed suspicious lesions during ductoscopy. Therefore, ductoscopy has a sensitivity for detecting malignancy of 71.4\% (95\% CI, 29.0\%-96.3\%), a specificity of 97.9\% (95\% CI, 94.0\%-99.6\%), and a negative predictive value of $98.6 \%$ (95\% CI, $95.6 \%-99.6 \%)$.

Table 4. Detection of Malignancy With Ductoscopy in Patients With PND Undergoing Ductoscopy

\begin{tabular}{lccc}
\hline & $\begin{array}{c}\text { Patient Had (Pre) } \\
\text { malignancy }\end{array}$ & $\begin{array}{c}\text { Patient Did Not Have } \\
\text { (Pre) malignancy }\end{array}$ & Total \\
\hline Ductoscopy showed suspicious lesions & 5 & 3 & 8 \\
Ductoscopy did not show suspicious lesions & 2 & 141 & 143 \\
Total & 7 & 144 & 151 \\
\hline
\end{tabular}

Abbreviation: PND = pathologic nipple discharge

\section{Complications}

Eleven $(5.1 \%)$ of the 215 patients with PND suitable for analysis experienced severe post procedural pain longer than 1 day, and 21 (9.7\%) patients had minor complaints of pain. The remaining $184(85.2 \%)$ did not experience any post procedural pain. There were no significant differences in pain perception between successful and unsuccessful ductoscopy procedures.

Five (2.3\%) of the 215 patients developed a (mild) mastitis after (attempted) ductoscopy requiring antibiotics. There were no significant differences in infection between successful and unsuccessful ductoscopy procedures.

In 1 case, a granulomatous mastitis was diagnosed post ductoscopy, finally requiring surgical incision and drainage of abscesses. This was the only serious complication found after ductoscopy. Because her follow-up time was less than 3 months, this case was left out of the final analysis regarding long-term follow-up after ductoscopy. 


\section{Discussion}

Ductoscopy is an established minimally invasive endoscopic technique that makes it possible to visualize the ductal tree in patients with PND. In recent years, it is also possible to endoscopically remove intraductal lesions during ductoscopy procedures with the use of an endobasket. The current retrospective study describes 215 patients with PND undergoing ductoscopy for further clinical validation. We found that interventional ductoscopy is able to detect (pre)malignant lesions and can prevent unnecessary diagnostic surgical procedures in 2 of 3 patients suffering from PND.

In this study, ductoscopy was considered successful when it was possible to visualize the ductal tree. However, previous studies define cannulation as a successful ductoscopy. ${ }^{20-22,27-30}$ This might explain why we experienced a slightly lower success rate compared with these previous studies. On the other hand, the proportion of successful visualization of the ducts $(70.2 \%)$ is higher in our study than previously described. ${ }^{28}$ As one might expect, we found that non-reducible nipple retraction and previous surgery of the mammilla are strong negative predictors for successful ductoscopy. Therefore, in the future, these patients should perhaps not be offered ductoscopy as a primary diagnostic tool for PND. Omitting these patients in our current series would have led to a successful ductoscopy rate of $77.7 \%$.

Currently, most women suffering from PND as their only complaint are offered surgery to rule out malignancy, even in the absence of radiologic suspicion of malignancy. Our study, however, shows that ductoscopy is a very useful tool in the diagnostic workup in these cases, preventing unnecessary surgery in a significant percentage of women with PND. Ninety-five of the 151 successful ductoscopy procedures revealed intraductal lesions causing PND (68 polypoid lesions, 19 non suspicious flat epithelial lesions including ductitis, 8 suspicious lesions, and 4 not otherwise specified). Forty-nine (51.6\%) of these lesions could be (partially) extracted using the endobasket, leaving room for improvement. Thirty-four (69.4\%) removed lesions were finally suitable for histologic analysis, which showed $33(97.1 \%)$ papillomas and $1(2.9 \%)$ case of normal tissue. Owing to its technical limitations, the endobasket is only useful as a tool in obtaining a histologic diagnosis in polypoid lesions. Because there are still no other suitable biopsy tools available yet for ductoscopic use, this remains a drawback in the success rate of ductoscopy. Effort should therefore be made to develop new small grasping biopsy tools suitable for use during ductoscopy procedures, making histologic diagnosis of all lesions found possible, including flat lesions. With such tools, the number of unnecessary surgical procedures in women suffering PND can probably be reduced even more.

There was a mean follow-up of 14.5 months, ranging from 3 months to 44.6 months. PND stopped without the need for surgery in 93 (43.2\%) patients, which is less than previously reported in the literature by Makita et al, who reported disappearance of PND 
in $85.1 \% .29$ However, they only reported on the cases in which an intraductal lesion was excised during ductoscopy, and they had a median follow-up of 5.5 years. In 18 cases in our series, PND stopped even after an unsuccessful ductoscopy, supporting the notion of the self-limiting nature of PND and the possible effect of ductal lavage in some patients, especially in ductectasia and/or ductitis or in the absence of a true intraductal lesion. This finding shows that careful selection of patients for surgery is adamant and that ductoscopy can be used as a useful selection tool.

Our main objective in introducing ductoscopy as a diagnostic tool in women with PND was reduction of the percentage of "unnecessary" surgery in these patients. In the present series, only $60(27.9 \%)$ patients underwent surgery after (attempted) ductoscopy (Figure

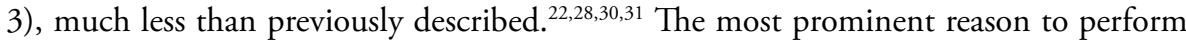
surgery was suspicious findings during ductoscopy. The most common indications for post-ductoscopy surgery were persistence and recurrence of PND. On the other hand, reassurance of the patient (ie, absence of a serious lesion) turned out to be important, in some cases even after unsuccessful ductoscopy.

The current study shows that ductoscopy is safe. The reported complications were post procedural pain $(14.8 \%)$ and infection $(2.3 \%)$. This is in line with previously published literature. $^{32}$

Eight $(3.7 \%)$ patients with PND and no suspicious radiologic or pathologic findings prior to ductoscopy were eventually diagnosed with DCIS after surgery. This is slightly less than the $5 \%$ to $10 \%$ we expected based on the literature. ${ }^{6,7}$ During ductoscopy, 8 patients with suspicious lesions were seen, of which 5 finally turned out to be (pre) malignant and 3 turned out to be benign after pathologic analysis. Two patients who turned out to have (pre)malignancy did not have suspicious findings during ductoscopy. However, in one of these patients, the DCIS was discovered by mere chance after breast reduction therapy almost 4 years after the ductoscopy. It is therefore difficult to assess whether the (pre)malignancy was missed during ductoscopy. The second patient with malignancy (invasive carcinoma) without suspicious ductoscopic findings underwent duct excision in which the histopathologic report was negative for (pre)malignancy. Only after subsequent surgery owing to persistent symptoms was (pre)malignancy diagnosed.

Sensitivity and specificity for the detection of malignancy was $71.4 \%$ (with a broad confidence interval) and $97.9 \%$, respectively. The broad CI of the sensitivity is explained by the low prevalence of (pre)malignancy. Additionally, the negative predictive value was 98.6\%. Sensitivity, specificity, and negative predictive values were similar to previously conducted research in patients with an increased risk for the development of breast cancer. ${ }^{33}$ All other results are in line with previous studies conducted. ${ }^{20-22,28,29,31}$ 
In recent years, MRI is sometimes used to find the cause of PND. However, small lesions are often missed, and it is difficult to differentiate benign from malignant lesions. Furthermore, outcome of an MRI has little effect on therapeutic choice for PND because a (surgical) biopsy is usually needed when a possible causing lesion is found. ${ }^{12,14,34}$ Sensitivity of MRI varies, when ultrasound and mammography are negative, from $40 \%$ to $86 \%$. The specificity of MRI to rule out breast cancer in patients with PND also ranges from $76 \%$ to $99 \%{ }^{12,35,36}$ Figure 4 shows a proposed flowchart for the indication of the usage of MRI and ductoscopy in the diagnostic process. Additionally, cytologic examination of PND has a low sensitivity for ruling out malignancy and may even lead to many falsepositive results. ${ }^{37,38}$ Our study not only shows that (interventional) ductoscopy has a high specificity and negative predictive value when it comes to the detection of malignancy, but it also has a therapeutic potential to stop the PND itself.

In our experience, ductoscopy is an easy to learn procedure for breast surgeons. Zagouri et al already described a learning curve of ductoscopy in ex vivo mastectomy specimens and suggest that an average surgeon requires 13 procedures to master this technique. ${ }^{39}$ We expect that the addition of the intervention (basket extraction) has no influence on the learning curve. Although we did not perform a formal cost analysis study comparing ductoscopy with duct excision surgery, we think it is safe to emphasize that using ductoscopy as a selection tool for surgery saves health care costs. Ductoscopy can be performed under local anesthesia in the outpatient clinic. It is a simple and relatively quick procedure, usually taking 15 to 30 minutes, which can be performed by a breast surgeon and only 1 trained nurse. Patients usually experience few or no side effects and can go back to work the same day or the next day. In our institution, the reimbursement for ductoscopy is one-half of the reimbursement for major duct excision, whereas in our hands, ductoscopy was able to select two-thirds of patients with PND in which surgery can safely be omitted. This being said, we can assume that even if a number of patients require surgery after ductoscopy, the total costs (ductoscopy and surgery) would probably be lower than if all patients with PND undergo surgery. However, a cost analysis study would be interesting to confirm these assumptions.

In conclusion, our study shows that ductoscopy is safe, with a high specificity and negative predictive value to detect (pre)malignancy and to treat PND. This makes ductoscopy a useful tool in deselecting women for major duct excision or microdochectomy, preventing the use of unnecessary surgery in many women with PND. 


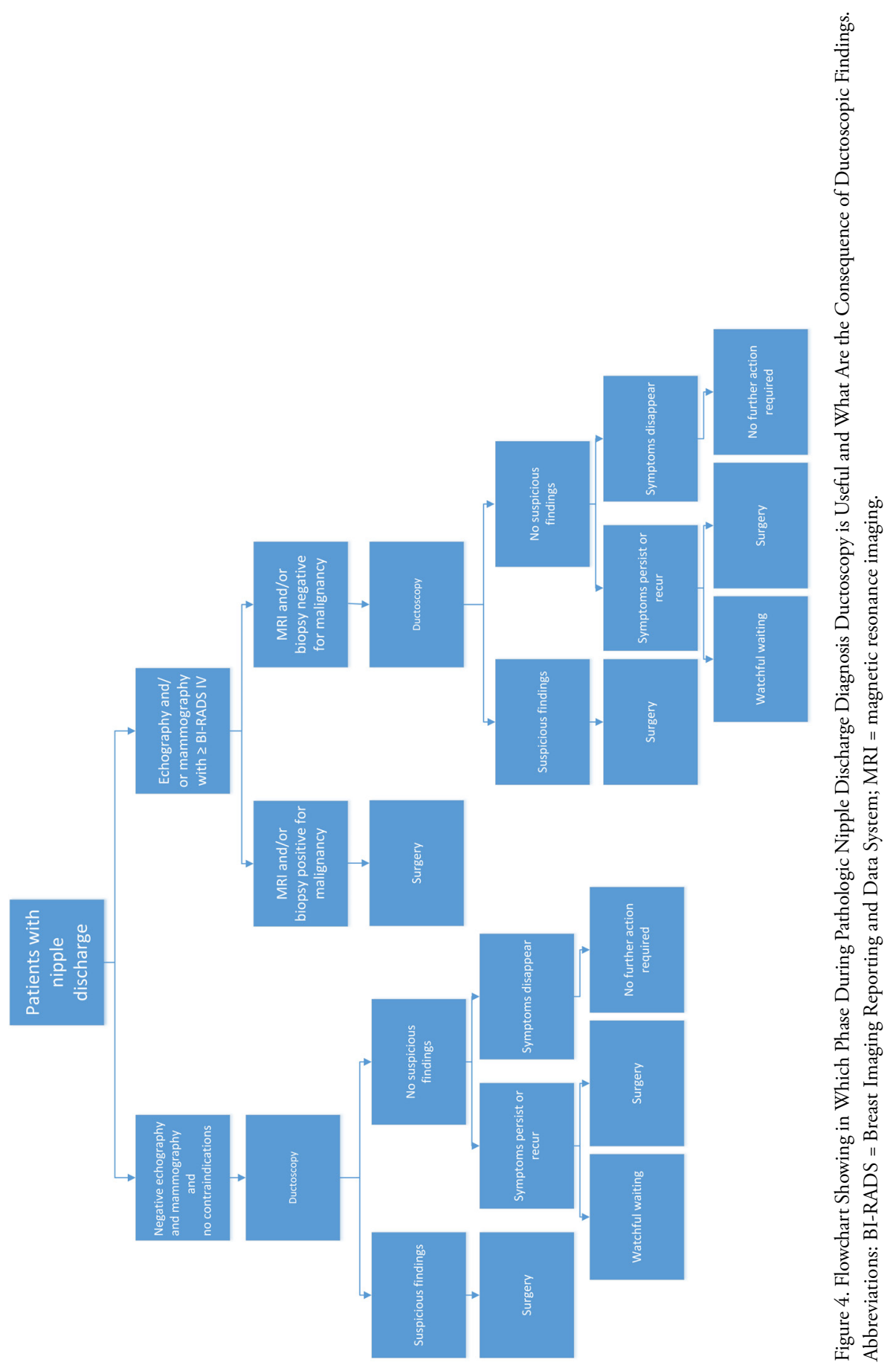




\section{References}

1. Hussain AN, Policarpio C, Vincent MT. Evaluating nipple discharge. Obstet Gynecol Surv. 2006;61(4):278-283. doi:0006254-200604000-00025 [pii]

2. Dixon JM, Mansel RE. ABC of breast diseases. Symptoms assessment and guidelines for referral. BMJ. 1994;309(6956):722-726.

3. Seltzer MH. Breast complaints, biopsies, and cancer correlated with age in 10,000 consecutive new surgical referrals. Breast J. 2004;10(2):111-117. doi:21284 [pii]

4. Santen RJ, Mansel R. Benign breast disorders. N Engl J Med. 2005;353(3):275-285. doi:353/3/275 [pii]

5. King TA, Carter KM, Bolton JS, Fuhrman GM. A simple approach to nipple discharge. Am Surg. 2000;66(10):960-966.

6. Galvin R, Joyce D, Downey E, Boland F, Fahey T, Hill AK. Development and validation of a clinical prediction rule to identify suspected breast cancer: a prospective cohort study. BMC Cancer. 2014;14:743. doi:10.1186/1471-2407-14-743 [doi]

7. Lesetedi C, Rayne S, Kruger D, Benn CA. Indicators of breast cancer in patients undergoing microdochectomy for a pathological nipple discharge in a middle-income country. J Surg Res. 2017;220:336-340. doi:S0022-4804(17)30428-6 [pii]

8. C. A, G.T. L, Alcock C, Layer GT. Predicting occult malignancy in nipple discharge. ANZ J Surg. 2010;80(9):646-649. doi:10.1111/j.1445-2197.2010.05270.x [doi]

9. Albrecht $\mathrm{C}$, Thele F, Grunwald S, et al. Nipple discharge: role of ductoscopy in comparison with standard diagnostic tests. Onkologie. 2013;36(1-2):12-16. doi:10.1159/000346639 [doi]

10. Bahl M, Baker JA, Greenup RA, Ghate S V. Diagnostic Value of Ultrasound in Female Patients With Nipple Discharge. AJRAmerican J Roentgenol. 2015;205(1):203-208. doi:10.2214/AJR.14.13354 [doi]

11. Sanders LM, Daigle M. The Rightful Role of MRI after Negative Conventional Imaging in the Management of Bloody Nipple Discharge. Breast J. 2016;22(2):209-212. doi:10.1111/tbj.12551 [doi]

12. van Gelder L, Bisschops RH, Menke-Pluymers MB, Westenend PJ, Plaisier PW. Magnetic resonance imaging in patients with unilateral bloody nipple discharge; useful when conventional diagnostics are negative? World J Surg. 2015;39(1):184-186. doi:10.1007/s00268-014-2701-1 [doi]

13. Paula IB de, Campos AM. Breast imaging in patients with nipple discharge. Radiol Bras. 2017. doi:10.1590/0100-3984.2016.0103

14. on Breast Imaging: EP, Lee SJ, Trikha S, et al. ACR Appropriateness Criteria((R)) Evaluation of Nipple Discharge. J Am Coll Radiol. 2017;14(5S):S138-S153. doi:S1546-1440(17)30137-0 [pii]

15. Sarakbi W Al, Worku D, Escobar PF, Mokbel K. Breast papillomas: current management with a focus on a new diagnostic and therapeutic modality. Int Semin Surg Oncol. 2006;3:1. doi:1477-7800-3-1 [pii]

16. Yamamoto D, Shoji T, Kawanishi H, et al. A utility of ductography and fiberoptic ductoscopy for patients with nipple discharge. Breast Cancer Res Treat. 2001;70(2):103-108.

17. Yamamoto D, Ueda S, Senzaki H, et al. New diagnostic approach to intracystic lesions of the breast by fiberoptic ductoscopy. Anticancer Res. 2001;21(6A):4113-4116.

18. Okazaki A, Okazaki M, Asaishi K, et al. Fiberoptic ductoscopy of the breast: a new diagnostic procedure for nipple discharge. Jpn J Clin Oncol. 1991;21(3):188-193.

19. Matsunaga T, Ohta D, Misaka T, et al. Mammary ductoscopy for diagnosis and treatment of intraductal lesions of the breast. Breast Cancer. 2001;8(3):213-221. 
20. Grunwald S, Heyer H, Paepke S, et al. Diagnostic value of ductoscopy in the diagnosis of nipple discharge and intraductal proliferations in comparison to standard methods. Onkologie. 2007;30(5):243-248. doi:100848 [pii]

21. Han Y, Li J, Han S, Jia S, Zhang Y, Zhang W. Diagnostic value of endoscopic appearance during ductoscopy in patients with pathological nipple discharge. BMC Cancer. 2017;17(1):300-303. doi:10.1186/s12885017-3288-3 [doi]

22. Kamali S, Bender O, Kamali GH, Aydin MT, Karatepe O, Yuney E. Diagnostic and therapeutic value of ductoscopy in nipple discharge and intraductal proliferations compared with standard methods. Breast Cancer. 2014;21(2):154-161. doi:10.1007/s12282-012-0377-7 [doi]

23. Jacobs VR, Kiechle M, Plattner B, Fischer T, Paepke S. Breast ductoscopy with a 0.55-mm miniendoscope for direct visualization of intraductal lesions. J Minim Invasive Gynecol. 2005;12(4):359-364. doi:S1553-4650(05)00274-8 [pii]

24. Ling H, Liu GY, Lu JS, et al. Fiberoptic ductoscopy-guided intraductal biopsy improve the diagnosis of nipple discharge. Breast J. 2009;15(2):168-175. doi:10.1111/j.1524-4741.2009.00692.x [doi]

25. Zhu X, Xing C, Jin T, Cai L, Li J, Chen Q. A randomized controlled study of selective microdochectomy guided by ductoscopic wire marking or methylene blue injection. Am J Surg. 2011;201(2):221-225. doi:10.1016/j.amjsurg.2010.03.011 [doi]

26. Moncrief RM, Nayar R, Diaz LK, Staradub VL, Morrow M, Khan SA. A comparison of ductoscopyguided and conventional surgical excision in women with spontaneous nipple discharge. Ann Surg. 2005;241(4):575-581. doi:00000658-200504000-00006 [pii]

27. Bender O, Balci FL, Yuney E, Akbulut H. Scarless endoscopic papillomectomy of the breast. Onkologie. 2009;32(3):94-98. doi:10.1159/000195694 [doi]

28. Waaijer L, van Diest PJ, Verkooijen HM, et al. Interventional ductoscopy in patients with pathological nipple discharge. Br J Surg. 2015;102(13):1639-1648. doi:10.1002/bjs.9950 [doi]

29. Makita M, Akiyama F, Gomi N, Iwase T. Mammary ductoscopy and watchful follow-up substitute microdochectomy in patients with bloody nipple discharge. Breast Cancer. 2016;23(2):242-251 doi:10.1007/s12282-014-0561-z [doi]

30. Yang X, Li H, Gou J, et al. The role of breast ductoscopy in evaluation of nipple discharge: a chinese experience of 419 patients. Breast J. 2014;20(4):388-393. doi:10.1111/tbj.12275 [doi]

31. Liu M, Guo G, Xie F, Wang S, Yang H, Wang S. Mammary ductoscopy and follow-up avoid unnecessary duct excision in patients with pathologic nipple discharge. J Surg Oncol. 2015;112(2):139-143. doi: $10.1002 /$ jso.23972 [doi]

32. Simpson JS, Connolly EM, Leong WL, et al. Mammary ductoscopy in the evaluation and treatment of pathologic nipple discharge: a Canadian experience. Can J surgeryJournal Can Chir. 2009;52(6):E245-8.

33. Gui G, Agusti A, Twelves D, et al. INTEND II randomized clinical trial of intraoperative duct endoscopy in pathological nipple discharge. Br J Surg. 2018;105(12):1583-1590. doi:10.1002/bjs.10990 [doi]

34. Eiada R, Chong J, Kulkarni S, Goldberg F, Muradali D. Papillary lesions of the breast: MRI, ultrasound, and mammographic appearances. AJRAmerican J Roentgenol. 2012;198(2):264-271. doi:10.2214/ AJR.11.7922 [doi]

35. Zacharioudakis K, Kontoulis T, Vella JX, et al. Can we see what is invisible? The role of MRI in the evaluation and management of patients with pathological nipple discharge. Breast Cancer Res Treat. 2019;178(1):115-120. doi:10.1007/s10549-019-05321-w [doi] 
36. Morrogh M, Morris EA, Liberman L, Borgen PI, King TA. The predictive value of ductography and magnetic resonance imaging in the management of nipple discharge. Ann Surg Oncol. 2007;14(12):33693377. doi:10.1245/s10434-007-9530-5 [doi]

37. Kooistra BW, Wauters C, van de Ven S, Strobbe L. The diagnostic value of nipple discharge cytology in 618 consecutive patients. Eur J Surg Oncol. 2009;35(6):573-577. doi:10.1016/j.ejso.2008.09.009 [doi]

38. Dolan RT, Butler JS, Kell MR, Gorey TF, Stokes MA. Nipple discharge and the efficacy of duct cytology in evaluating breast cancer risk. Surgeon. 2010;8(5):252-258. doi:10.1016/j.surge.2010.03.005 [doi]

39. Zagouri F, Sergentanis TN, Giannakopoulou G, et al. Breast ductal endoscopy: how many procedures qualify? BMC Res Notes. 2009;2:115. doi:10.1186/1756-0500-2-115 [doi] 
Interventional ductoscopy as an alternative for major duct excision or microdochectomy in women 


\section{Chapter 4}

\section{Detection of breast cancer precursor lesions by autofluorescence ductoscopy}

L. Waaijer M.D. Filipe

J.M. Simons

C.C. van der Pol

T. de Boorder

P.J. van Diest

A.J. Witkamp 


\section{Abstract}

\section{Purpose}

Autofluorescence is an image enhancement technique used for the detection of cancer precursor lesions in pulmonary and gastrointestinal endoscopy. This study evaluated the feasibility of addition of autofluorescence to ductoscopy for the detection of intraductal breast cancer precursor lesions.

\section{Methods}

An autofluorescence imaging system, producing real-time computed images combining fluorescence intensities, was coupled to a conventional white light ductoscopy system. Prior to surgery, ductoscopy with white light and autofluorescence was evaluated under general anaesthesia in women scheduled for therapeutic or prophylactic mastectomy. Endoscopic findings in both modes were compared, marked and correlated with histology of the surgical specimen.

\section{Results}

Four breast cancer patients and five high-risk women, with a median age of 47 years (range 23-62) were included. In autofluorescence mode, two intraductal lesions were seen in two breast cancer patients, which had an increase in the red-to-green fluorescence intensity compared with the surrounding tissue. One lesion had initially been missed by white light ductoscopy but was clearly visible in subsequent autofluorescence mode. One endoscopic finding was classified as suspicious by white light, but was negative in autofluorescence mode and showed normal histology.

\section{Conclusions}

This study demonstrates for the first time the in vivo feasibility of autofluorescence ductoscopy to detect pathologically confirmed breast cancer precursor lesions in both breast cancer patients and high-risk women that were occult under white light. 


\section{Introductions}

Breast cancer is, with 523,000 new cases a year, the most common type of cancer and accounts for 138,000 deaths a year in Europe. ${ }^{1}$ ereditary breast cancer accounts for up to $5-10 \%$ of all breast cancers with two high-penetrance genes (BRCA1 and BRCA2) responsible for about $16 \%$ of the familial risk of breast cancers, associated with a $60-80 \%$ lifetime risk of developing breast cancer. ${ }^{2-5}$ Currently, the ultimate prevention in these women is bilateral prophylactic mastectomy. ${ }^{6}$ Consequently, this means that $20-40 \%$ of patients undergo mastectomies without signs of malignancy. Unfortunately, mastectomies are accompanied by complications along with serious cosmetic and psychological consequences. ${ }^{7,8}$

Most breast cancers are thought to arise from the ductal epithelium. ${ }^{9,10}$ An appealing approach would be to target breast cancer precursors originating from the epithelial lining of the breast ducts through ductoscopy. This is a minimally invasive microendoscopic technique, which makes real-time visualisation of the milk ducts of the breast possible. Ductoscopy is currently performed under local anaesthesia at the outpatient clinic, and is currently mainly applied as an additional diagnostic tool in the work-up of women suffering from pathological nipple discharge (PND) without suspicious radiological findings. ${ }^{11-18}$ Different studies show that ductoscopy can accurately detect intraductal lesions causing PND before or during duct excision. ${ }^{19-23}$ The role of ductoscopy in breast-cancer screening and breast conservation surgery has yet to be fully defined, ${ }^{24}$ but the first studies using autofluorescence in ductoscopy indicated the feasibility and the possibility to detect malignant lesions..$^{25,26}$ The former study was an ex vivo study, the latter a technical in vivo feasibility study in three patients not aimed at detecting lesions, and without taking material for pathological evaluation. (Pre)malignant epithelial lesions show an aberrant pattern under fluorescent light by which they become detectable, as is already known from the airways, larynx and colon. ${ }^{27-29}$ However, the breast ductal system had not been evaluated before by autofluorescence to detect pathologically confirmed precursor lesions.

From a prospective feasibility study in patients affected by breast cancer and in women with a known mutation in $B R C A 1$ or $B R C A 2$, we report for the first time the in vivo feasibility of autofluorescence to detect (white light occult) breast cancer precursor lesions by autofluorescence ductoscopy, confirmed by histology of the subsequently performed mastectomy. 


\section{Methods}

\section{Patients}

A prospective observational phase II cohort study was conducted in adult women who underwent either therapeutic or prophylactic mastectomy in the University Medical Center Utrecht, The Netherlands, between October 2014 and May 2015.

Two cohorts were included. The therapeutic cohort consisted of 4 female patients undergoing a mastectomy for recently diagnosed invasive breast cancer or ductal carcinoma in situ (DCIS). The prophylactic cohort consisted of 5 women undergoing prophylactic mastectomy for increased risk of breast cancer. The first cohort hypothetically carries multiple precursor lesions and serves as a reference for the autofluorescence ductoscopy technique; the second is the index population that will provide information about the diagnostic value of this technique in high-risk patients.

Patients with previous surgery or radiotherapy of the breast were excluded. This study was approved by the Institutional Review Board of the UMC Utrecht. All patients provided written informed consent.

\section{White light and autofluorescence ductoscopy}

Study procedures were conducted immediately prior to mastectomy, and were all performed under sterile conditions and under general anaesthesia in the operation room.

First, a saline solution was injected around the nipple to cause thrust and thereby exposing the orifices of the milk ducts. Salivary duct probes (Karl Storz, Tuttlingen, Germany) size 0000 to 1 and an obturator (Polydiagnost GmbH, Pfaffenhofen, Germany) were used for dilatation of one of the duct orifices in the nipple. The introduction port (SoLexNipple-Expander ${ }^{\oplus}$, Polydiagnost) or a custom-made introduction port compatible with the Storz endoscope was placed into the duct orifice through which the ductoscope was introduced. A $0.55 \mathrm{~mm}$ optic (LaDuScope T-flex, Polydiagnost) was inserted in a $1.15 \mathrm{~mm}$ outer diameter Polyshaft (PD-DS-1015, Polydiagnost) or a Storz miniature endoscope (Erlangen, Karl Storz) with incorporated fiberoptic light transmission and an outer diameter of $1.1 \mathrm{~mm}$, was used. Both devices are semiflexible and have a separate irrigation channel for saline-infusion, and a working channel (diameter $0.45 \mathrm{~mm}$ ).

The ductoscope was coupled via a custom-made eyepiece to an autofluorescence endoscopic imaging system $\left(\right.$ OncoLIFE$^{\oplus}$, Xillix Technologies Corporation, British Columbia, Canada, now Pinpoint ${ }^{\oplus}$, Novadaq Technologies Corporation, Ontario, Canada). Method of operation was described previously by Douplik et al. ${ }^{25}$ Briefly, white-light and autofluorescence images were recorded using $6.3-\mathrm{mW}$ broadband light 
and 5.3-mW blue band (390 to $450 \mathrm{~nm}$ ), respectively. In other organs, premalignant tissues have a reduced green autofluorescence relative to normal tissues when excited by blue light; normal tissue appears as cyan, while abnormal tissue is shown red coloured. ${ }^{28,30}$ In autofluorescence mode, the central 16-12 pixels are averaged over four frames and continuously displayed as a numerical color value (NCV). The higher the NCV, the lower the autofluorescence intensity, which has been associated with neoplasia ${ }^{31}$.

First, standard (white-light mode) ductoscopy was performed. Whenever suspicious findings were encountered, we switched to autofluorescence mode by a hand switch or foot pedal. When no suspicious findings were encountered under white light, the entire duct was examined by autofluorescence ductoscopy.

White light findings were classified as normal (no visual abnormality) or abnormal (irregularity of the ductal lining such as redness, hypervascularity, swelling, thickening, as well as nodular or polypoid lesions). In autofluorescence, a green colour was classified as normal, while areas showing red colour with decreased autofluorescence were classified as abnormal. In autofluorescence mode NCV values were continuously monitored.

In the first seven patients, the ductoscopy procedure was performed via one single duct orifice, to limit operation time. In the 8th case, multiple ducts were examined. In the therapeutic cohort ductoscopic exploration was performed in the breast quadrant containing malignancy to encounter the previously established lesion. In the prophylactic cohort the duct orifice that was easiest to cannulate was chosen and only one breast was examined. In case of an abnormality in the studied duct, 1-2 $\mathrm{ml}$ colour marker (sterile Black Eye Endoscopic marker ${ }^{\mathrm{Tm}}$, The Standard, Korea) was placed through the working channel of the ductoscope after removal of the optic, to facilitate precise histological correlation. Distance of the lesion to the nipple was also recorded. When no abnormalities were found, the most extensively examined duct was marked. Images of ductal abnormalities were recorded. All ductoscopy procedures were performed by the same physicians (AW, CP).

Following the ductoscopy procedure a conventional (therapeutic or prophylactic) mastectomy was performed. 


\section{Pathology}

Mastectomy specimens were submitted fresh to pathology, where the margins of the specimen were inked with non-black colours to avoid interference with the intraductal dye-mark. The specimen was sliced in 5-mm slices, and scrutinized for the marked area and macroscopic lesions. At the level of the colour marker, the specimen was totally embedded at a transversal plane to acquire a trans sectional view of the duct. All tissue was formalin fixed and used for routine histological evaluation using conventional haematoxylin-eosin (HE) staining. At the level of the tumour the specimen was embedded according to standard procedure.

All intraductal abnormities were described. Assessment of the surgical specimens was performed by one dedicated breast pathologist (PD) blinded to the endoscopic results.

\section{Follow up}

Decisions regarding postoperative treatment with adjuvant chemotherapy or hormonal therapy were made according to usual protocols based on patients' risk category and based on the tumour characteristics, size and stage.

\section{Evaluation and analysis}

Primary endpoint was the technical feasibility, determined by cannulation success and findings of intraductal abnormalities. Endoscopic findings in white light, autofluorescence and NCVs were correlated to final histology of the surgical specimen. Differences in endoscopic findings under white light and autofluorescence ductoscopy were described. 


\section{Results}

Table 1 shows the patient and imaging characteristics of the 9 included patients. Duct cannulation and subsequent ductoscopic exploration were successful in 8 of 9 (89\%) women. In one patient undergoing prophylactic mastectomy cannulation failed due to narrow duct orifices.

Table 1. Ductoscopic, radiologic, and patient characteristics of the two cohorts

\begin{tabular}{lll}
\hline & $\begin{array}{l}\text { Therapeutic Mastectomy } \\
\mathbf{n}=\mathbf{5}\end{array}$ & $\begin{array}{l}\text { Prophylactic Bilateral } \\
\text { Mastectomy } \mathbf{n}=\mathbf{4}\end{array}$ \\
\hline Mean age (range), years & 50 (range, 29-62) & 44 (range, 23-61) \\
Spontaneous nipple discharge & 0 & 0 \\
Ductoscopy side N* & & \\
Left & 3 & 3 \\
Right & 2 & 1 \\
\hline
\end{tabular}

*In bilateral prophylactic mastectomy, the ductoscopy was performed unilateral, in the most accessible duct orifice for cannulation. Reported here is the ductoscopy side where cannulation was successful

Ductoscopic examination time, from cannulation to termination of the procedure ranged from 15 to $45 \mathrm{~min}$ (mean, $28.3 \mathrm{~min}$ ). This is in line with conventional ductoscopy, additional time for autofluorescence examination ranged from 5 to $8 \mathrm{~min}$.

\section{Breast cancer patients}

In four of five breast cancer patients solitary $(n=2)$ or multiple $(n=2)$ intraductal abnormalities were visualised in with light and/or autofluorescence mode. Table 2 shows the characteristics of the lesions found. Normal appearing ducts in white light coloured green in autofluorescence mode, corresponding with low NCVs (Fig. 1a). In patient 1, a deposition in the lining of a duct was seen with autofluorescence mode, but not by white light mode, at around $4-5 \mathrm{~cm}$ from the nipple. In this patient, the first cannulation attempt was unsuccessful. The pathology report showed that DCIS was also found in the nipple area. 


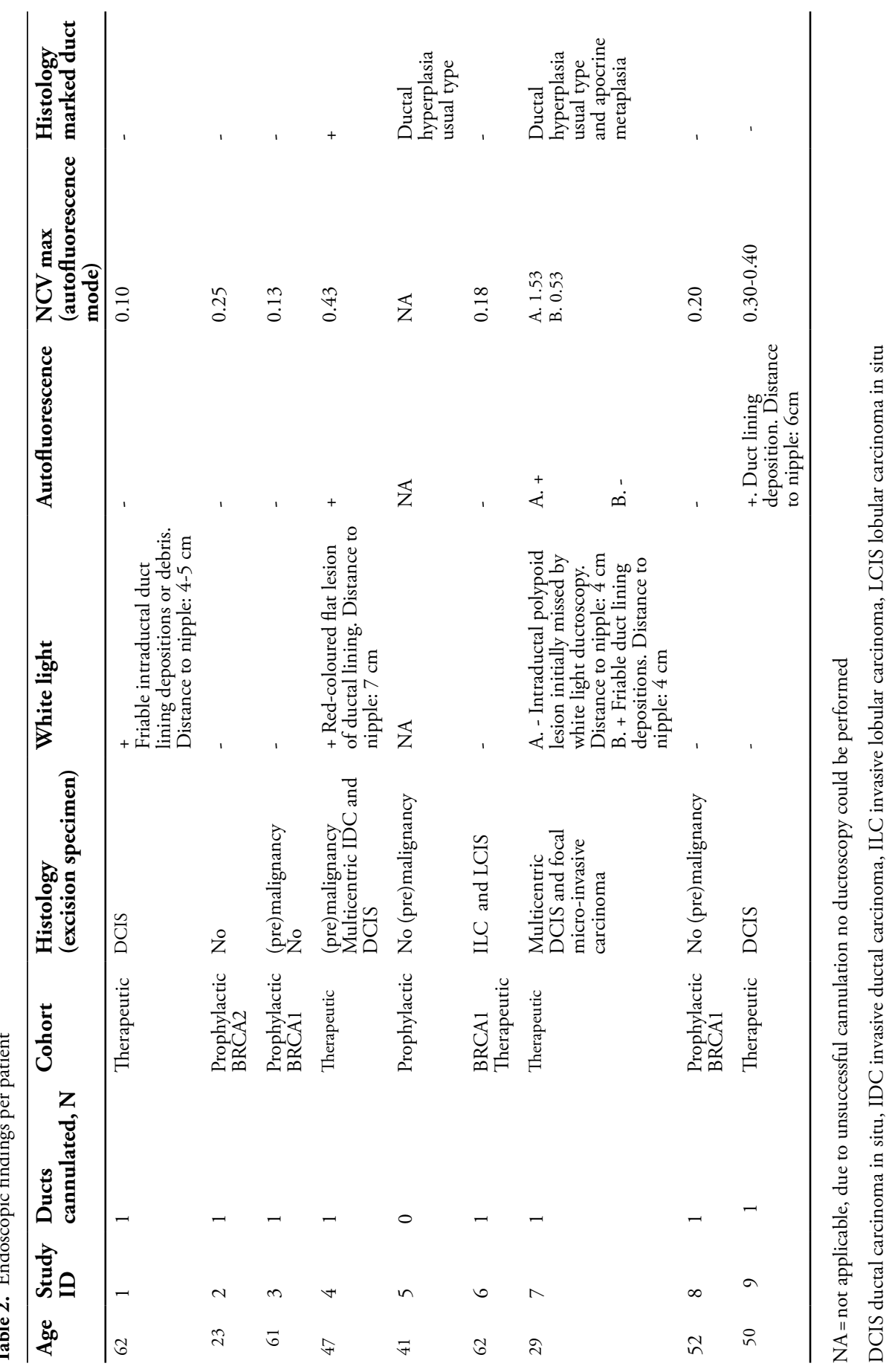


Fig 1 .

A

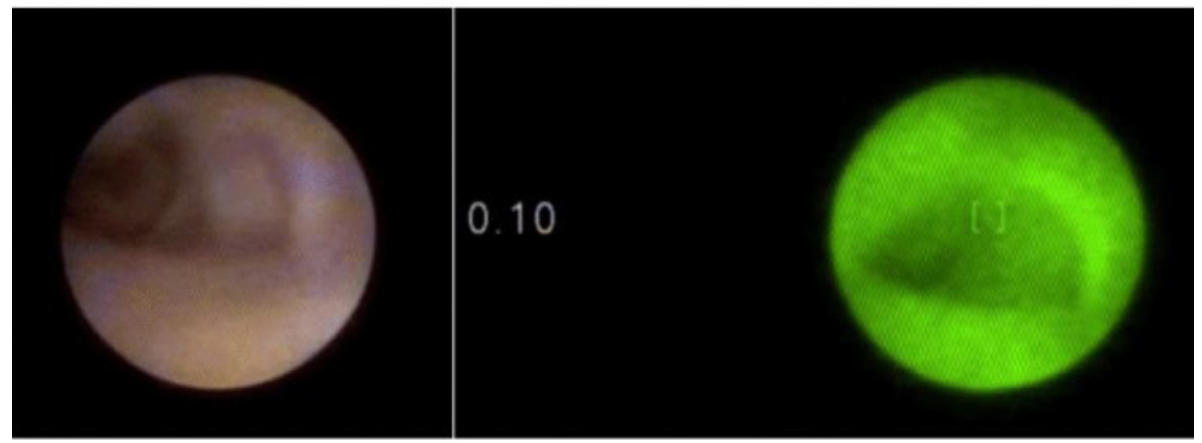

\section{B}

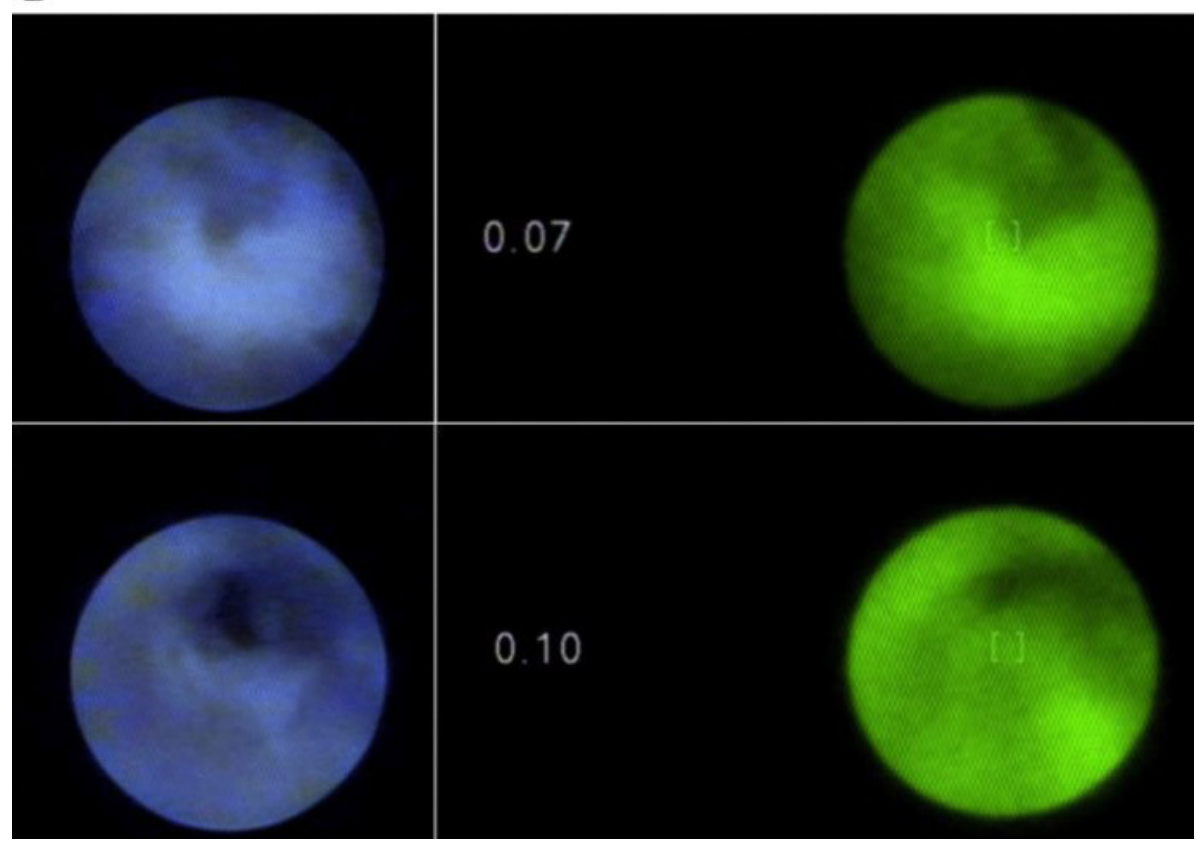

a Left: normal-appearing duct in white light. Right: same duct in autofluorescence mode, showing green colour and corresponding low NCV.

b Upper and lower left: intraductal abnormalities in white light. Upper and lower right: showing the same abnormalities in autofluorescence mode, with green colour and corresponding low NCV 
In a second breast cancer patient (patient 4), three friable intraductal abnormalities (debris) were visualised in white light mode at $7 \mathrm{~cm}$ from the nipple. In autofluorescence mode these abnormalities where green-coloured with NCVs $<0.10$ (Fig. 1b). Histology of the examined duct showed the intraductal marker, confirming the correct localization. Histology showed no abnormalities.

In patient 6, a haemorrhagic, red-coloured epithelial lesion was seen under white light, showing normal green colour in autofluorescence mode with maximum NCVs of 0.43 (Fig. 2). Histology of the duct showed the intraductal marker, confirming the correct localization, with ductal epithelial hyperplasia and epithelial damage.

Fig 2.

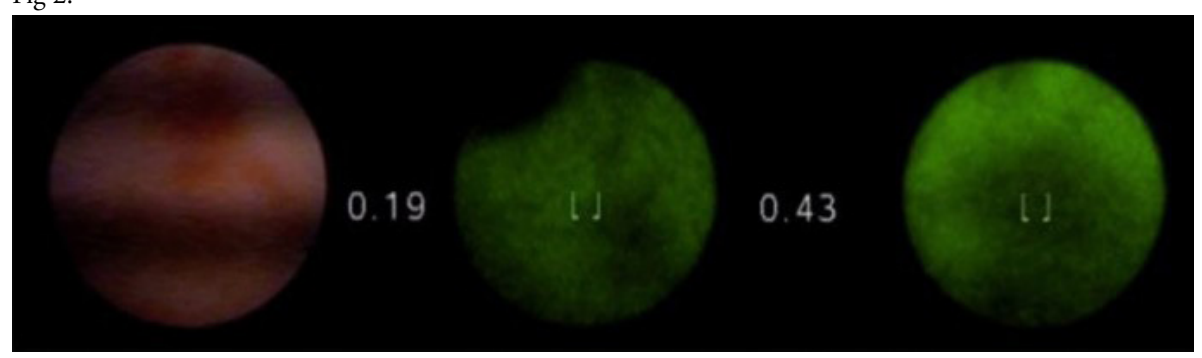

Flat epithelial lesion in white light. On middle and right: showing the same lesion in autofluorescence mode, showing a red-coloured contrast with the green ductal lining and showing increased NCVs (0.43)

In patient 7 , an intraductal polypoid lesion appearing as irregular protrusion into the ductal lumen, with colours similar to the surrounding ductal tissue, was initially missed by white light ductoscopy at $4 \mathrm{~cm}$ from the nipple. In autofluorescence mode, the same lesion was displayed as a red-coloured intraductal polypoid lesion clearly contrasting the surrounding ductal tissue, with maximum NCVs of 1.53 (Fig. 3a). In the same ductal tree, another abnormality was visualised (Fig. 3b); friable ductal wall adhesions seen in white light, showed no clear red-colour in autofluorescence mode but did show maximum NCVs of 0.53 . Histology of the examined ductal system showed the intraductal black dye-marking with both apocrine metaplasia and ductal hyperplasia (Fig. 3c). Since the dye-marker coloured the complete ductal system, more precise correlation of the two separate intraductal abnormalities was not possible. 
Fig 3 .

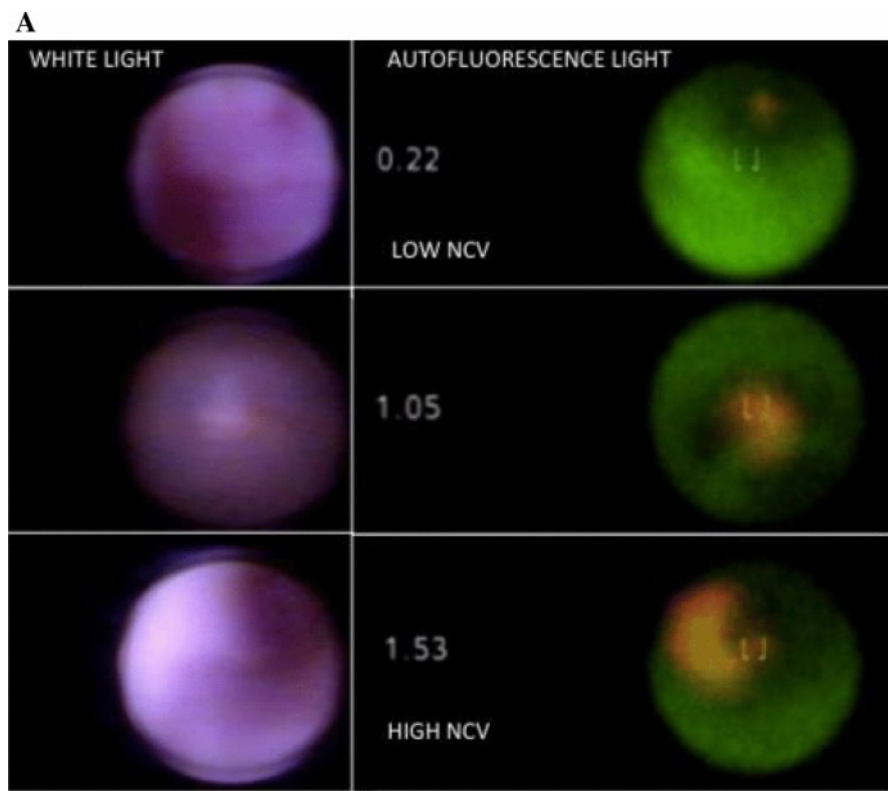

B

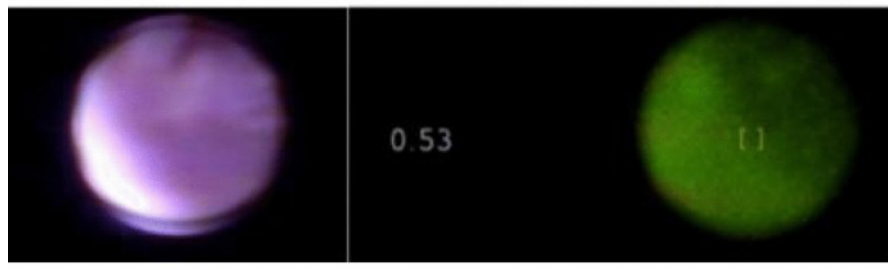

$\mathbf{C}$

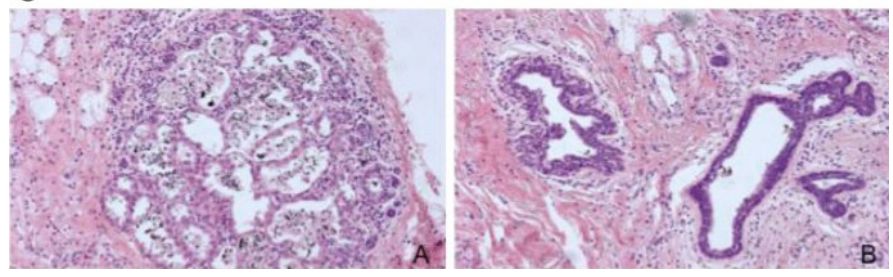

a Ductoscopic images taken immediately prior to therapeutic mastectomy for multicentric DCIS grade 3. White light ductoscopy (left). Upper: showing an intraductal puffy aspect of the duct wall with ductal asymmetry (oval shape). Middle: intraductal polypoid lesion, not clearly identifiable from the duct wall. Lower: close up of the lesion. Autofluorescence ductoscopy (right). Upper: focusing on the normal, green coloured, duct wall. NCV shows corresponding low values (0.22). Middle: focusing on the intraductal red coloured intraductal abnormality, NCV shows corresponding high NCV values (1.05). Lower: Close-up of the same lesion, high NCVs (1.53).

b Endoscopic view in the same ductal system. Autofluorescence and white light image of the high NCVs on a small area of duct lining. Friable lesions seen in white light, showing no clear red-colour but increased NCVs.

$\mathbf{c}$ Histology of the examined ductal system of figures $\mathbf{a}$ and $\mathbf{b}$ show the intraductal black dye marking and apocrine metaplasia and ductal hyperplasia of the usual type. Since the dye-marker colours the complete examined ductal tree, precise correlation of the two separate intraductal abnormalities shown in $\mathbf{a}$ and $\mathbf{b}$ is not possible 
In the last breast cancer patient (patient 9) the white light ductoscopy showed a possible red-coloured epithelial lesion with NCVs of 3.38 at $6 \mathrm{~cm}$ from the nipple. By autofluoresences mode, a clear epithelial lesion was seen (elevated NCV) which was microscopically identified as DCIS and lobular neoplasia were found outside the area of the ductoscopic lesion.

\section{Prophylactic patients}

In none of the patients undergoing prophylactic mastectomy abnormalities were found with either white light or autofluorescence ductoscopy. This was confirmed by histology of the studied ducts, showing no abnormalities.

\section{Follow up}

Following ductoscopy, all patients underwent mastectomy. All resections were radical. Sentinel node biopsy was performed in all patients undergoing therapeutic surgery. In one patient the sentinel node contained a micro-metastasis. Adjuvant systemic therapy and radiation therapy was given in one patient. Median follow-up after surgery was 4 months (range 2-6). In all patients of the prophylactic cohort, immediate reconstruction was performed. In one patient undergoing prophylactic mastectomy with tissue expanders for subsequent reconstruction, bilateral necrosis of skin and nipple occurred, for which surgical necrotectomy was performed. No other complications occurred. Perforation of one or more ducts during ductoscopy occurred in five patients. Although this may limit endoscopic view $(n=1)$, it is without consequence for the patient. Subsequently inserted intraductal marker macroscopically showed extraductal diffusion from the ducts with perforations, without impairing microscopic localization (Fig. 4). 
Fig. 4
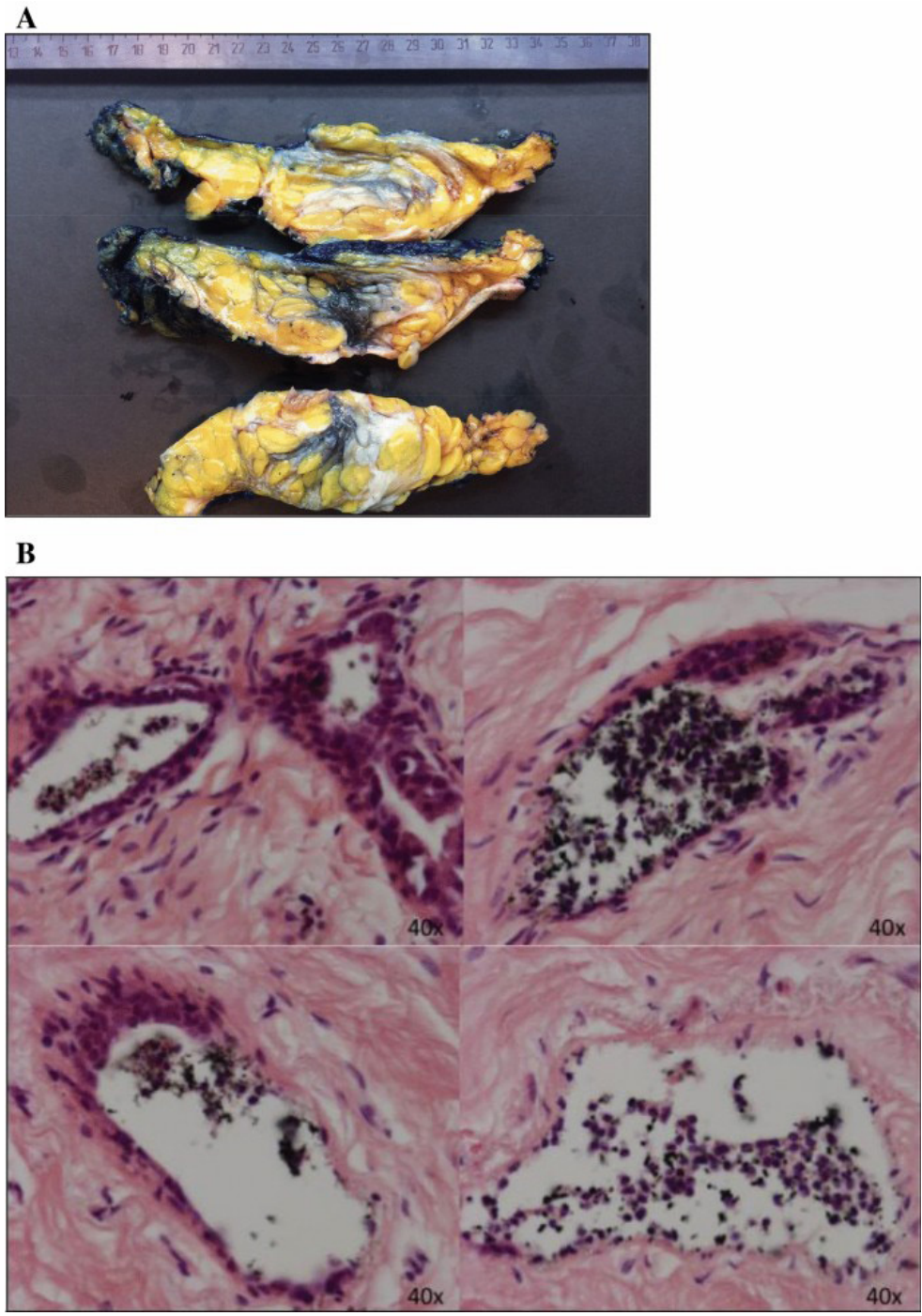

a Marking of the ductoscopic visualized duct in a patient undergoing prophylactic mastectomy for BRCA1. At (white light and autofluorescence) ductoscopy no abnormalities were found. Intraductal marking with Black Eye Endoscopic marker ${ }^{\mathrm{rm}}$ showed macroscopically clear localization for embedding. b Microscopically, the intraductal marking is easily traceable and the extravasation is not visible. The ductal marking shows the histological changes previously reported to be caused by ductoscopy: periductal clefts, epithelial detachment and epithelial loss ${ }^{32}$ 


\section{Discussion}

This exploratory study indicates that autofluorescence ductoscopy is technically feasible in both breast cancer patients and high-risk women, with successful cannulation in 8 of $9(89 \%)$ women. Autofluorescence ductoscopy was capable of identifying ductal hyperplasia and apocrine metaplasia and showed to be correctly negative in histological normal ducts. The used technique of intraductal marking with endoscopic dye-marker resulted in accurate macro- and microscopic localization of the studied duct (Fig. 5).

Fig. 5
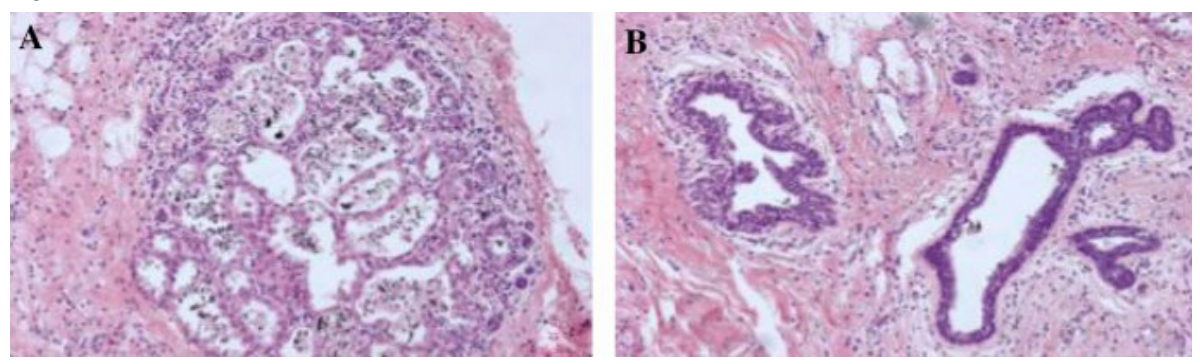

Histology of the examined ductal system of figure $\mathbf{a}$ and $\mathbf{b}$ show the intraductal black dye marking and apocrine metaplasia and ductal hyperplasia. Since the dye-marker colours the complete examined the ductal tree, precise correlation of the two separate intraductal abnormalities shown in a and $\mathrm{b}$ is not possible.

Although autofluorescence ductoscopy was positive in ductal hyperplasia and apocrine metaplasia, breast cancer precursor lesions were not found in this pilot study. The used autofluorescence settings are optimal for early-stage disease in other hollow organs such as the bronchus and gastro-intestinal tract, but further optimization of the imaging parameters may be necessary to increase specificity for premalignant lesions in the breast. Intraductal debris was seen in the lumen of the normal duct, which can be misinterpreted as cancer, appropriately appeared negative in autofluorescence mode, demonstrating the differentiating potential of autofluorescence ductoscopy in white light endoscopically suspect lesions. The continuous irrigation of saline solution not only ensured that the milk ducts remained open but also that the debris was washed away. Consequently, we did not encounter any interference with the image.

No lesions were found in women undergoing prophylactic mastectomy for high risk of breast cancer. These negative endoscopic findings were confirmed by histology. To evaluate the diagnostic accuracy of autofluorescence ductoscopy for the screening of highrisk women, a larger cohort in whom a number of premalignant lesions are present needs to be studied and prospectively followed with ductoscopy. 
In previous studies using ductal lavage to guide risk-reducing strategies in high-risk women, (conventional) ductoscopy has already been suggested as a risk assessment tool for high-risk women. ${ }^{33,34}$ Danforth et al. compared ductoscopic findings with ductal lavage cytology in the contralateral high-risk breasts of breast cancer patients and visualised intraductal lesions in $83 \%$ of the ducts with atypical cytology. ${ }^{35}$ On the contrary, in a study of ductal lavage cytology in asymptomatic, high-risk patients, poor concordance with histology was found and ductoscopy added little to this evaluation ${ }^{36}$ Although ductal lavage fails in yielding adequate specimens for reliable cytological diagnosis, ${ }^{37}$ diagnosis by the use of proteomic biomarkers in serum or methylation in nipple aspiration fluid or ductal lavage forms a promising alternative. ${ }^{38-42}$ Methylation of specific genes is known as an early hallmark of carcinogenesis and can be detected in an only small amount of DNA, providing a potential method for early tumour detection. Addition of autofluorescence ductoscopy could possibly assist in visualising and locating early lesions. Together with the currently being studied novel intraductal treatment modalities e.g. via intraductal targeted therapy by RNA interference, ${ }^{43}$ intraductal chemotherapy ${ }^{44}$ or intraductal laser ablation $^{45}$, this would form an appealing approach for early detection and treatment.

Some previous studies showed the success of ductoscopy in finding the intraductal lesion causing PND before or during duct excision..$^{20,21,46}$ In recent years, a biopsy tool was introduced that can be used through the working channel of the ductoscope enabling intraductal biopsy or removal of found lesions. ${ }^{18,23,47}$ This so-called interventional ductoscopy has already been described before as a safe alternative for classic open surgery in patients with $\mathrm{PND},{ }^{18,46,47}$ but wider implementation requires further validation studies.

There are several more issues that need to be addressed. Most breast cancer arises from the terminal ductolobular unit (TDLU), where the ducts are narrow. ${ }^{48}$ For ductoscopic examination of these TDLUs further minimization of diameter is needed. More challenging could be the anatomy of the breast, with the discrepancy between a number of duct and orifices in the nipple due to several ducts arising in the same cleft of the nipple. ${ }^{49}$ Complete endoscopic examination will be difficult and sampling error could occur. Here, biomarker evaluation, e.g. RNA analysis of PND in ductal lavage or nipple aspiration fluid could be of additional value.

Also, the current techniques of ductoscopic diagnostic tissue acquisition are far from optimal. The 'basket'-intervention device is only feasible in polypoid lesions ${ }^{18,23,47,50,51}$ and intraductal biopsy devices are not commercially available. ${ }^{52,53}$ For histologic correlation of the endoscopic findings of this study were dependant of dye-marker injected through the working channel with subsequent surgical excision. This procedure caused marking of a complete single ductal system, precluding correlation of multiple lesions within one duct. Therefore, the development of a commercially available biopsy device suitable for superficial epithelial lesions remains much warranted. 
Our results may have clinical implications for another patient group. Due to the association with breast cancer, numerous women with pathologic nipple discharge and negative imaging undergo exploratory surgery to rule out malignancy and to treat symptoms, despite breast cancer being found in a minor 3-7\% in this patient group. 18,47,54-57 Ductoscopy has been used as a diagnostic modality to rule out malignancy, but while some studies reported a significant correlation, ${ }^{58,59}$ others found no specific data except for gross morphological abnormalities such as papillomas. ${ }^{22,60-62} \mathrm{~A}$ recent network meta-analysis showed that white ductoscopy has a high specificity (98\%) but low sensitivity $(44 \%)$ for the detection of breast cancer in patients with pathological nipple discharge with no radiological suspicion for malignancy. ${ }^{63}$ Since intraductal lesions (such as papillomas) can be removed with relative ease and histopathologically analysed, ${ }^{18,47}$ autofluoresence might not be of added value in these cases. However, autofluorescence may help to increase the sensitivity of ductoscopy for the detection of breast cancer of lesions of the ductal wall, improving risk assessment and correlated treatment decisions.

It is estimated that $20-40 \%$ of BRCA1/2 patients who would never develop breast cancer are grossly overtreated with preventive mastectomies. ${ }^{2-6}$ Stratification within these highrisk group remains elusive and more sensitive screening methods are warranted, although primary prevention with less radical treatment methods would be the ultimate solution. The current study shows that autofluorescence ductoscopy could be a feasible tool to stratify these high-risk groups. However, larger groups are necessary to determine the diagnostic performance of auto fluorescence ductoscopy. Nonetheless, we do expect auto fluorescence to be a valuable addition to ductoscopy for the detection of breast cancer precursor lesions since auto fluorescence has shown to be superior to white light endoscopies for the detection of precursor lesions in other cancer types of epithelial origin. ${ }^{64-67}$

In conclusion, ductoscopy with the addition of autofluorescence is feasible in diagnosing intraductal breast lesions and could possibly increase specificity for endoscopic morphologically suspicious findings. However, this technique needs to be optimized and studied more intensively before it will be applicable in clinical practice. 


\section{References}

1. Ferlay J, Colombet M, Soerjomataram I, et al. Cancer incidence and mortality patterns in Europe: Estimates for 40 countries and 25 major cancers in 2018. Eur J Cancer. 2018;103:356-387. doi:S0959-8049(18) 30955-9 [pii]

2. Ford D, Easton DF, Stratton M, et al. Genetic heterogeneity and penetrance analysis of the BRCA1 and BRCA2 genes in breast cancer families. The Breast Cancer Linkage Consortium. Am J Hum Genet. 1998;62(3):676-689. doi:S0002-9297(07)63848-8 [pii]

3. Tung N, Battelli C, Allen B, et al. Frequency of mutations in individuals with breast cancer referred for BRCA1 and BRCA2 testing using next-generation sequencing with a 25-gene panel. Cancer. 2015;121(1):25-33. doi:10.1002/cncr.29010 [doi]

4. Robson ME, Bradbury AR, Arun B, et al. American Society of Clinical Oncology Policy Statement Update: Genetic and Genomic Testing for Cancer Susceptibility. J Clin Oncol. 2015;33(31):3660-3667. doi:10.1200/JCO.2015.63.0996 [doi]

5. Couch FJ, Shimelis H, Hu C, et al. Associations Between Cancer Predisposition Testing Panel Genes and Breast Cancer. JAMA Oncol. 2017;3(9):1190-1196. doi:10.1001/jamaoncol.2017.0424 [doi]

6. Ludwig KK, Neuner J, Butler A, Geurts JL, Kong AL. Risk reduction and survival benefit of prophylactic surgery in BRCA mutation carriers, a systematic review. Am J Surg. 2016;212(4):660-669. doi:S0002-9610(16)30348-8 [pii]

7. Osman F, Saleh F, Jackson TD, Corrigan MA, Cil T. Increased postoperative complications in bilateral mastectomy patients compared to unilateral mastectomy: an analysis of the NSQIP database. Ann Surg Oncol. 2013;20(10):3212-3217. doi:10.1245/s10434-013-3116-1 [doi]

8. Eisen A, Rebbeck TR, Wood WC, Weber BL. Prophylactic surgery in women with a hereditary predisposition to breast and ovarian cancer. J Clin Oncol. 2000;18(9):1980-1995. doi:10.1200/JCO.2000.18.9.1980 [doi]

9. Wellings SR. A hypothesis of the origin of human breast cancer from the terminal ductal lobular unit. Pathol Res Pract. 1980;166(4):515-535. doi:S0344-0338(80)80248-2 [pii]

10. Bean GR, Bryson AD, Pilie PG, et al. Morphologically normal-appearing mammary epithelial cells obtained from high-risk women exhibit methylation silencing of INK4a/ARF. Clin Cancer Res. 2007;13(22 Pt 1):6834-6841. doi:13/22/6834 [pii]

11. Yamamoto D, Shoji T, Kawanishi H, et al. A utility of ductography and fiberoptic ductoscopy for patients with nipple discharge. Breast Cancer Res Treat. 2001;70(2):103-108.

12. Yamamoto D, Ueda S, Senzaki H, et al. New diagnostic approach to intracystic lesions of the breast by fiberoptic ductoscopy. Anticancer Res. 2001;21(6A):4113-4116.

13. Okazaki A, Okazaki M, Asaishi K, et al. Fiberoptic ductoscopy of the breast: a new diagnostic procedure for nipple discharge. Jpn J Clin Oncol. 1991;21(3):188-193.

14. Matsunaga T, Ohta D, Misaka T, et al. Mammary ductoscopy for diagnosis and treatment of intraductal lesions of the breast. Breast Cancer. 2001;8(3):213-221.

15. Grunwald S, Heyer H, Paepke S, et al. Diagnostic value of ductoscopy in the diagnosis of nipple discharge and intraductal proliferations in comparison to standard methods. Onkologie. 2007;30(5):243-248. doi: 100848 [pii]

16. Han Y, Li J, Han S, Jia S, Zhang Y, Zhang W. Diagnostic value of endoscopic appearance during ductoscopy in patients with pathological nipple discharge. BMC Cancer. 2017;17(1):300-303. doi:10.1186/s12885017-3288-3 [doi] 
17. Kamali S, Bender O, Kamali GH, Aydin MT, Karatepe O, Yuney E. Diagnostic and therapeutic value of ductoscopy in nipple discharge and intraductal proliferations compared with standard methods. Breast Cancer. 2014;21(2):154-161. doi:10.1007/s12282-012-0377-7 [doi]

18. Filipe MD, Waaijer L, van der Pol CC, van Diest P, Witkamp AJ. Interventional ductoscopy as an alternative for major duct excision or microdochectomy in women suffering pathological nipple discharge: a single centre experience. Clin Breast Cancer. January 2020. doi:10.1016/j.clbc.2019.12.008

19. Jacobs VR, Kiechle M, Plattner B, Fischer T, Paepke S. Breast ductoscopy with a 0.55-mm miniendoscope for direct visualization of intraductal lesions. J Minim Invasive Gynecol. 2005;12(4):359-364. doi:S1553-4650(05)00274-8 [pii]

20. Ling H, Liu GY, Lu JS, et al. Fiberoptic ductoscopy-guided intraductal biopsy improve the diagnosis of nipple discharge. Breast J. 2009;15(2):168-175. doi:10.1111/j.1524-4741.2009.00692.x [doi]

21. Zhu X, Xing C, Jin T, Cai L, Li J, Chen Q. A randomized controlled study of selective microdochectomy guided by ductoscopic wire marking or methylene blue injection. Am J Surg. 2011;201(2):221-225. doi:10.1016/j.amjsurg.2010.03.011 [doi]

22. Moncrief RM, Nayar R, Diaz LK, Staradub VL, Morrow M, Khan SA. A comparison of ductoscopyguided and conventional surgical excision in women with spontaneous nipple discharge. Ann Surg. 2005;241(4):575-581. doi:00000658-200504000-00006 [pii]

23. Bender O, Balci FL, Yuney E, Akbulut H. Scarless endoscopic papillomectomy of the breast. Onkologie. 2009;32(3):94-98. doi:10.1159/000195694 [doi]

24. Tang SS, Twelves DJ, Isacke CM, Gui GP. Mammary ductoscopy in the current management of breast disease. Surg Endosc. 2011;25(6):1712-1722. doi:10.1007/s00464-010-1465-4 [doi]

25. Douplik A, Leong WL, Easson AM, Done S, Netchev G, Wilson BC. Feasibility study of autofluorescence mammary ductoscopy. J Biomed Opt. 2009;14(4):44036. doi:10.1117/1.3210773 [doi]

26. Jacobs VR, Paepke S, Schaaf H, Weber BC, Kiechle-Bahat M. Autofluorescence ductoscopy: a new imaging technique for intraductal breast endoscopy. Clin Breast Cancer. 2007;7(8):619-623. doi:S1526-8209(11)70750-9 [pii]

27. Ogihara T, Watanabe H, Namihisa A, Kobayashi O, Miwa H, Sato N. Clinical experience using a real time autofluorescence endoscopy system in the gastrointestinal tract. Diagn Ther Endosc. 1999;5(2):119-124. doi:10.1155/DTE.5.119 [doi]

28. Ikeda N, Honda H, Hayashi A, etal. Earlydetection of bronchial lesions using newly developed videoendoscopybased autofluorescence bronchoscopy. Lung Cancer. 2006;52(1):21-27. doi:S0169-5002(06)00018-3 [pii]

29. van der Heijden EH, Hoefsloot W, van Hees HW, Schuurbiers OC. High definition bronchoscopy: a randomized exploratory study of diagnostic value compared to standard white light bronchoscopy and autofluorescence bronchoscopy. Respir Res. 2015;16:33-37. doi:10.1186/s12931-015-0193-7 [doi]

30. Chhajed PN, Shibuya K, Hoshino H, et al. A comparison of video and autofluorescence bronchoscopy in patients at high risk of lung cancer. Eur Respir J. 2005;25(6):951-955. doi:25/6/951 [pii]

31. Lee P, van den Berg RM, Lam S, et al. Color fluorescence ratio for detection of bronchial dysplasia and carcinoma in situ. Clin Cancer Res. 2009;15(14):4700-4705. doi:10.1158/1078-0432.CCR-08-1644 [doi]

32. Tresserra F, Fabregas R, Torrent J, et al. Morphologic changes in breast biopsies after duct endoscopy. Breast. 2001;10(2):149-154. doi:10.1054/brst.2000.0172 [doi]

33. Hollingsworth AB, Singletary SE, Morrow M, et al. Current comprehensive assessment and management of women at increased risk for breast cancer. Am J Surg. 2004;187(3):349-362. doi:10.1016/j. amjsurg.2003.12.025 [doi] 
34. B. P, K. M, Pereira B, Mokbel K. Mammary ductoscopy: Past, present, and future. Int J Clin Oncol. 2005;10(2):112-116. doi:10.1007/s10147-004-0403-7

35. Jr DND, Abati A, Filie A, et al. Combined breast ductal lavage and ductal endoscopy for the evaluation of the high-risk breast: a feasibility study. J Surg Oncol. 2006;94(7):555-564. doi:10.1002/jso.20650 [doi]

36. Cyr AE, Margenthaler JA, Conway J, et al. Correlation of ductal lavage cytology with ductoscopy-directed duct excision histology in women at high risk for developing breast cancer: a prospective, single-institution trial. Ann Surg Oncol. 2011;18(11):3192-3197. doi:10.1245/s10434-011-1963-1 [doi]

37. Loud JT, Thiebaut AC, Abati AD, et al. Ductal lavage in women from BRCA1/2 families: is there a future for ductal lavage in women at increased genetic risk of breast cancer? Cancer Epidemiol Biomarkers Prev. 2009;18(4):1243-1251. doi:10.1158/1055-9965.EPI-08-0795 [doi]

38. Antill YC, Mitchell G, Johnson SA, et al. Gene methylation in breast ductal fluid from BRCA1 and BRCA2 mutation carriers. Cancer Epidemiol Biomarkers Prev. 2010;19(1):265-274. doi:10.1158/1055-9965.EPI09-0359 [doi]

39. Suijkerbuijk KP, van der Wall E, Vooijs M, et al. Molecular analysis of nipple fluid for breast cancer screening. Pathobiology. 2008;75(2):149-152. doi:10.1159/000123853 [doi]

40. de Noo ME, Deelder A, van der Werff M, Ozalp A, Mertens B, Tollenaar R. MALDI-TOF serum protein profiling for the detection of breast cancer. Onkologie. 2006;29(11):501-506. doi:95933 [pii]

41. Mertens BJ, Noo ME De, Tollenaar RA, Deelder AM. Mass spectrometry proteomic diagnosis: enacting the double cross-validatory paradigm. J Comput Biol. 2006;13(9):1591-1605. doi:10.1089/cmb.2006.13.1591 [doi]

42. Suijkerbuijk KP, van Diest PJ, van der Wall E. Improving early breast cancer detection: focus on methylation. Ann Oncol Off J Eur Soc Med Oncol. 2011;22(1):24-29. doi:10.1093/annonc/mdq305 [doi]

43. Brock A, Krause S, Li H, et al. Silencing HoxA1 by intraductal injection of siRNA lipidoid nanoparticles prevents mammary tumor progression in mice. Sci Transl Med. 2014;6(217):217ra2. doi:10.1126/ scitranslmed.3007048 [doi]

44. de Groot JS, van Diest PJ, van Amersfoort M, et al. Intraductal cisplatin treatment in a BRCA-associated breast cancer mouse model attenuates tumor development but leads to systemic tumors in aged female mice. Oncotarget. 2017;8(37):60750-60763. doi:10.18632/oncotarget.18490 [doi]

45. de Boorder T, Waaijer L, van Diest PJ, Witkamp AJ. Ex vivo feasibility study of endoscopic intraductal laser ablation of the breast. Lasers Surg Med. 2018;50(2):137-142. doi:10.1002/lsm.22745

46. Makita M, Akiyama F, Gomi N, Iwase T. Mammary ductoscopy and watchful follow-up substitute microdochectomy in patients with bloody nipple discharge. Breast Cancer. 2016;23(2):242-251. doi:10.1007/s12282-014-0561-z [doi]

47. Waaijer L, van Diest PJ, Verkooijen HM, et al. Interventional ductoscopy in patients with pathological nipple discharge. Br J Surg. 2015;102(13):1639-1648. doi:10.1002/bjs.9950 [doi]

48. Wellings SR, Jensen HM, Marcum RG. An atlas of subgross pathology of the human breast with special reference to possible precancerous lesions. J Natl Cancer Inst. 1975;55(2):231-273.

49. Rusby JE, Brachtel EF, Michaelson JS, Koerner FC, Smith BL. Breast duct anatomy in the human nipple: three-dimensional patterns and clinical implications. Breast Cancer Res Treat. 2007;106(2):171-179. doi:10.1007/s10549-006-9487-2 [doi]

50. Kamali S, Kamali GH, Akan A, Simsek S, Bender O. Use of ductoscopy as an additional diagnostic method and its applications in nipple discharge. Minerva Chir. 2014;69(2):65-73. doi:R06Y2014N02A0065 [pii] 
51. Balci FL, Feldman SM. Interventional ductoscopy for pathological nipple discharge. Ann Surg Oncol. 2013;20(10):3352-3354. doi:10.1245/s10434-013-3181-5 [doi]

52. Matsunaga T, Misaka T, Hosokawa K, et al. Intraductal approach to the detection of intraductal lesions of the breast. Breast Cancer Res Treat. 2009;118(1):9-13. doi:10.1007/s10549-008-0203-2 [doi]

53. Hunerbein M, Raubach M, Gebauer B, Schneider W, Schlag PM. Ductoscopy and intraductal vacuum assisted biopsy in women with pathologic nipple discharge. Breast Cancer Res Treat. 2006;99(3):301-307. doi:10.1007/s10549-006-9209-9 [doi]

54. Vargas HI, Vargas MP, Eldrageely K, Gonzalez KD, Khalkhali I. Outcomes of clinical and surgical assessment of women with pathological nipple discharge. Am Surg. 2006;72(2):124-128.

55. Seltzer MH. Breast complaints, biopsies, and cancer correlated with age in 10,000 consecutive new surgical referrals. Breast J. 2004;10(2):111-117. doi:21284 [pii]

56. Gray RJ, Pockaj BA, Karstaedt PJ. Navigating murky waters: a modern treatment algorithm for nipple discharge. Am J Surg. 2007;194(6):850-855. doi:S0002-9610(07)00721-0 [pii]

57. Dillon MF, Nazri SRM, Nasir S, et al. The role of major duct excision and microdochectomy in the detection of breast carcinoma. BMC Cancer. 2006;6:164. doi:1471-2407-6-164 [pii]

58. Makita M, Akiyama F, Gomi N, Iwase T, Kasumi F, Sakamoto G. Endoscopic and histologic findings of intraductal lesions presenting with nipple discharge. Breast J. 2006;12(5 Suppl 2):S210-7. doi:TBJ324 [pii]

59. Denewer A, El-Etribi K, Nada N, El-Metwally M. The role and limitations of mammary ductoscope in management of pathologic nipple discharge. Breast J. 2008;14(5):442-449. doi:10.1111/j.15244741.2008.00620.x [doi]

60. Kamali S, Bender O, Aydin MT, Yuney E, Kamali G. Ductoscopy in the evaluation and management of nipple discharge. Ann Surg Oncol. 2010;17(3):778-783. doi:10.1245/s10434-009-0820-y [doi]

61. Louie LD, Crowe JP, Dawson AE, et al. Identification of breast cancer in patients with pathologic nipple discharge: does ductoscopy predict malignancy? Am J Surg. 2006;192(4):530-533. doi:S0002-9610(06)00450-8 [pii]

62. Dietz JR, Crowe JP, Grundfest S, Arrigain S, Kim JA. Directed duct excision by using mammary ductoscopy in patients with pathologic nipple discharge. Surgery. 2002;132(4):582-588. doi:S0039606002001368 [pii]

63. Filipe MD, Patuleia SIS, de Jong VMT, Vriens MR, van Diest PJ, Witkamp AJ. A network meta-analysis for the diagnostic approach to pathological nipple discharge. Clin Breast Cancer. June 2020. doi:10.1016/j. clbc.2020.05.015

64. Jin NY, Wang K, Wei SQ, et al. Diagnostic value of autofluorescence imaging combined with narrow band imaging in intraepithelial neoplasia of Barrett's esophagus. J BUON Off J Balk Union Oncol. 2015;20(2):399-405.

65. Singh R, Mei SC, Sethi S. Advanced endoscopic imaging in Barrett's oesophagus: a review on current practice. World J Gastroenterol. 2011;17(38):4271-4276. doi:10.3748/wjg.v17.i38.4271 [doi]

66. Moriichi K, Fujiya M, Sato R, et al. Back-to-back comparison of auto-fluorescence imaging (AFI) versus high resolution white light colonoscopy for adenoma detection. BMC Gastroenterol. 2012;12:75. doi:10.1186/1471-230X-12-75 [doi]

67. Thakur A, Gao L, Ren H, Yang T, Chen T, Chen M. Descriptive data on cancerous lung lesions detected by auto-fluorescence bronchoscope: A five-year study. Ann Thorac Med. 2012;7(1):21-25. doi:10.4103/18171737.91559 [doi] 
Detection of breast cancer precursor lesions by autofluorescence ductoscopy 



\section{Chapter 5}

Feasibility of narrow band imaging ductoscopy, intraductal biopsy and intraductal laser ablation In patients with pathological nipple discharge and patients with a high-risk for the development of breast cancer: a study protocol 


\section{Abstract}

\section{Introduction}

Ductoscopy is a minimally invasive endoscopic technique that enables direct intraductal visualization. Ductoscopy is often used to diagnose and sometimes treat women with pathological nipple discharge (PND) without radiological signs of malignancy. Malignancy accounts for around 5\% of cases while the remaining cases are benign papillomas and duct ectasia in this patient group. The challenges of ductoscopy lie in low sensitivity for the detection of malignancy, the lack of a proper intraductal biopsy device and good treatment of small papilloma lesions.

Narrow band imaging (NBI) is a visualisation technique that filters (red) light improving sensitivity and specificity of endoscopic techniques such as bronchoscopy and colonoscopy. Therefore, NBI could improve the sensitivity of ductoscopy.

Intraductal laser ablation has been shown to be feasible in the ex vivo setting. This technique could potentially be useful could potentially for intraductal lesions that cannot be removed. The aim of this study is to determine the in vivo feasibility of NBI ductoscopy, intraductal biopsy and intraductal laser ablation.

\section{Methods}

Treatment arm A are patients who are referred to for ductoscopy (patients with PND without radiological suspicion for malignancy). In this group, patients first undergo ductoscopy followed by NBI ductoscopy. When a lesion is seen, it is removed with an intraductal biopsy device developed by the Technical University of Delft (TU Delft). When lesion extraction is no (completely) possible, intraductal laser ablation is performed.

Treatment arm B are patient who are referred for breast surgery (both preventive and therapeutic). Prior to surgery, patients undergo ductoscopy followed by NBI ductoscopy. If a lesion is seen, intraductal biopsy is performed.

Findings of ductoscopy, NBI ductoscopy, intraductal biopsy and pathological analysis after (possible) surgery are compared. Additionally, patients in treatment arm A are followed to determine whether PND stopped and/or whether patients underwent duct excision surgery.

\section{Discussion}

Enhanced ductoscopy could prevent unnecessary surgeries in patients with PND. Additionally, ductoscopy may help to safely postpone preventive mastectomy in patients with an increased risk 


\section{Introduction}

Breast cancer is, with 523,000 new cases a year, the most common type of common type of cancer and accounts for 138,000 deaths a year in Europe. ${ }^{1}$ Hereditary breast cancer accounts for up to $5-10 \%$ of all breast cancers with two high-penetrance genes (BRCA1 and BRCA2) responsible for about $16 \%$ of the familial risk of breast cancers, associated with a $60-80 \%$ lifetime risk of developing breast cancer. ${ }^{2-5}$ Currently, the ultimate prevention in these women is bilateral prophylactic mastectomy. ${ }^{6}$ Consequently, this means that $20-40 \%$ of patients undergo mastectomies without signs of malignancy. Unfortunately, mastectomies are accompanied by complications along with serious cosmetic and psychological consequences. ${ }^{7,8}$

Pathological nipple discharge (PND) is defined as unilateral, spontaneous and bloody or serous discharge, usually arising from a single duct orifice of the nipple. After pain and palpable lumps, PND is the third most common breast-related complaint. ${ }^{9}$ PND is often associated with breast cancer and accounts for 3-5\% of surgical breast clinic referrals. ${ }^{10-13}$ However, the most common causes of PND are benign (duct ectasias and intraductal papillomas). ${ }^{14,15}$

Ductoscopy is a minimally invasive micro-endoscopic technique providing real-time visualization of the milk ducts of the breast. Ductoscopy is performed under local anaesthesia at the outpatient clinic and is currently used as a diagnostic tool in the workup of women suffering from PND. ${ }^{16-23}$ Previous studies and a meta-analysis show that ductoscopy is a useful tool in finding intraductal lesions causing PND (benign and malignant) before or during duct excision. ${ }^{24-26}$

Ductoscopy shows to have a sensitivity of $71.4 \%$ and specificity of $97.9 \%$ for the detection of breast cancer in patients with PND without radiological signs of malignancy. ${ }^{27}$ Adding auto fluorescent imaging to ductoscopy has been described to attempt to increase the sensitivity for breast cancer of ductoscopy in ex-vivo studies. ${ }^{28,29}$ However, there currently no clinical studies describing the diagnostic performance of auto fluorescent ductoscopy for the detection breast cancer. Nevertheless, there are currently other technologies that are widely used to increase the diagnostic performance for (pre)malignancy of other (endo)scopic procedures, such as narrow-band imaging (NBI). ${ }^{30}$

\section{Narrow Band Imaging}

$\mathrm{NBI}$ is an imaging technique for endoscopic diagnostic medical tests, where light of specific blue and green wavelengths is used to enhance the detail of certain aspects of the surface of the mucosa. A special filter is electronically activated by a switch in the endoscope leading to the use of ambient light of wavelengths of $415 \mathrm{~nm}$ (blue) and 540 $\mathrm{nm}$ (green). ${ }^{30}$ Peak light absorption of haemoglobin occurs at these wavelengths, blood 
vessels will contrast compared to other tissue, allowing for their improved visibility and in the improved identification of other surface structures. ${ }^{31}$ In gastrointestinal endoscopy, NBI has found use in the identification of Barrett's oesophagus.${ }^{31} \mathrm{NBI}$ is also used for the identification of pit patterns to classify colorectal polyps and tumours ${ }^{32}$ and in the identification of atypical dysplastic cells in the colon of patients with ulcerative colitis. 33 Additionally, in cystoscopy, which is the examination of the urinary bladder with an endoscope, NBI is useful in differentiating between benign and malignant cells. ${ }^{34}$ NBI has also been tested in the in ex vivo settings. ${ }^{35,36}$ However, there are currently no studies conducted in which NBI is applied during ductoscopy. It is reasonable to think NBI might be useful since (pre)malignancy is known to show different patterns of vascularisation (including neovascularisation and/or angiogenesis) compared to healthy breast tissue. ${ }^{37-41}$ Furthermore, histopathological confirmation of ductoscopic findings is necessary in order to determine whether a patient has (pre)cancerous lesions.

\section{Intraductal biopsy tool}

The current intraductal biopsy tool (endobasket) is not suitable to perform intraducal biopsies and are not always able to extract the pappilomas causing PND. ${ }^{23,27}$ Therefore, new intraductal biopsy tools are warranted. In cases of remnants of the papilloma causing the PND, other techniques could be used to ensure that PND is treated, such as laser ablation.

\section{Intraductal laser ablation}

Laser ablation techniques are widely used in medicine and have proven to be save and able to evaporate (pre)malignant lesions. ${ }^{42,43}$ The applicability of laser ablation was studied and showed to be feasible for intraductal use in an ex vivo setting. ${ }^{44}$ However, there are currently no studies showing the feasibility of in vivo intraductal laser ablation. 


\section{Methods/Design}

\section{Objective}

The primary aim is to study the feasibility of performing NBI ductoscopy, new biopsy tool and intraductal laser ablation in patients undergoing breast surgery and patients undergoing ductoscopy due to PND. Secondary objectives of this study are correlation of the different modes of imaging (white (regular) ductoscopy and NBI ductoscopy) to the histopathological analysis of the intraductal biopsy. Additionally, in the patients undergoing surgery, the different modes of imaging histopathological analysis of the intraductal biopsy will be compared to the histopathological analysis of the surgical specimen. Furthermore, this study will assess whether PND resolves after the patients undergo intraductal laser ablation.

\section{Study design}

This study is a phase II, monocentre trial to assess the feasibility of NBI ductoscopy, intraductal biopsy and intraductal laser ablation in patients undergoing ductoscopy due to PND and in patients undergoing breast surgery (preventive of therapeutic). This trial starts in September 2020.

Patients with PND without radiological signs of malignancy $(\operatorname{arm} \mathrm{A})$ are already planned for ductoscopy. In this study, these patients undergo also NBI ductoscopy, intraductal biopsy and intraductal biopsy. If suspicious findings are seen with (NBI) ductoscopy and/or determined with intraductal biopsy histopathology, patients will be planned for surgery. Patients planned for therapeutic or preventive breast surgery (arm B) will undergo ductoscopy followed by NBI ductoscopy, intraductal biopsy and intraductal laser ablation directly prior to surgery (Figure 1). 
Part 1 - Chapter 5

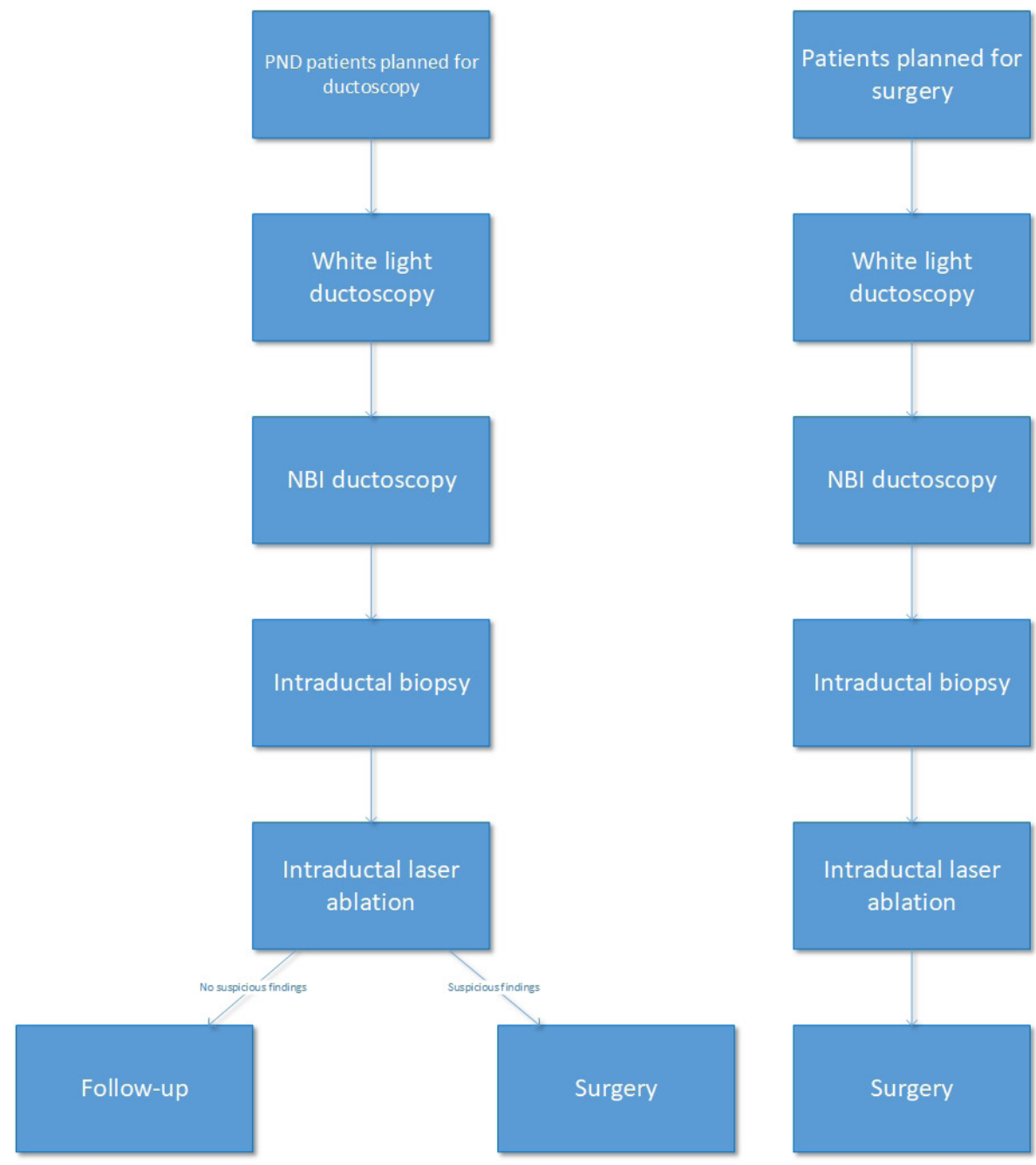

Figure 1. Study flowchart. PND = pathologic nipple discharge, NBI = narrow-band imaging. 


\section{Study population}

In order to be eligible to participate in this study, a subject must meet all of the following criteria:

1. Arm A: patients planned for ductoscopy

a. Patients with unilateral PND

b. No radiological suspicion for malignancy

c. Referred to the UMC Utrecht for ductoscopy

2. Arm B: patients planned for surgery

a. Preventive mastectomy

b. Therapeutic surgeries

A potential subject who meets any of the following criteria will be excluded from participation in this study for both groups:

1. Under the age of 18

2. Not being able to sign an informed consent

3. History of breast surgery at the affected breast

4. History of radiotherapy of the breast of thorax

5. Pregnancy

\section{Follow-up}

Follow-up will only be performed in arm A for 1 year. If (white light and/or NBI) ductoscopy and/or histopathological analysis of intraductal biopsy are suspicious for malignancy, patients will be scheduled for surgery. Patients might also choose for surgery if symptoms of PND persist despite the lack of suspicious findings. The follow-up moments will be 2 weeks, 3 months, 6 months and 1 year after ductoscopy. 


\section{Study endpoints and analysis}

\section{Primary endpoint}

Primary endpoint of this study is determining the feasibility of NBI ductoscopy, intraductal biopsy and intraductal laser ablation. Feasibility of intraductal biopsy will be considered successful when histopathological analysis is possible.

\section{Secondary endpoints}

Secondary endpoints are:

- Presence of PND symptoms over time (arm A only).

- Correlation of histopathological analysis of intraductal biopsy with histopathological analysis of surgical specimen in arm B and the operated patients of arm A.

- Correlation of white light ductoscopy and NBI to histopathological analysis of surgical specimen (all patients of arm A and only operated patients of arm B) and/ or intraductal biopsy.

\section{Statistical analysis}

Descriptive statistics will be used to describe patient and treatment characteristics of the study population. Continuous data will be described with mean along with standard deviation (SD), or with median and interquartile range (IQR), depending on the distribution. Mann-Whitney- $U$ test or $t$ test will be used to test differences between two groups of continuous data, depending on the distribution. Differences between categorical data will be assessed with Chi-Square test or Fisher's exact test depending on the number in cells. Subgroup analysis of arm A and arm B will also be performed. P-values below 0.05 will be considered significant.

All statistical calculations will be performed using RStudio 1.1 .456 (with $\mathrm{R}$ version: x64 3.6.2). Visualization of plots will be performed using the ggplot2 package. 


\section{Treatment strategies}

\section{Intervention in arm A}

Patients included in the study must have signed the informed consent. The surgeon will perform standard (white light) ductoscopy as described in previous studies in the outpatient clinic ${ }^{23,27,45}$. First, the surgeon identifies the lactiferous duct by pressing the nipple. Oxytocin nose spray 30 minutes prior to the procedure will be given to patients who do not have spontaneous PND at the time of the procedure. Next, the nipple will be disinfected with 70\% ethanol and 1\% lidocaine will used for local anaesthesia of the nipple. Salivary duct probe (size 0000 to 1; Karl Storz, Tuttlingen, Germany) and an obturator (Polydiagnost, Pfaffenhofen, Germany) will be used to widen the affected duct. This will be followed by the placement of a port through which the ductoscope can be introduced (SoLex nipple expander; Polydiagnost). Ductoscopy will be performed using a 6000-pixel 0.55-mm optic (LaDuScope T-flex; Polydiagnost) (Figure 2, top) and a Polyshaft (1.15-mm outer diameter, PD-DS-1015; Polydiagnost). Video processing is performed with OTV-S190 (Olympus) and the light source (white light and NBI) will come from CLV-S190 (Olympus), as seen in Figure 3. Visualization of the ductal tree starts at the affected duct. The surgeon will explore the major ducts in an orderly fashion until the ducts become too narrow to pass. Continuous saline irrigation into the ductal tree through the polyshaft is paramount to keep the ducts from collapsing. Ductoscopy will considered successful when a thorough examination of the afflicted ductal tree was possible. The visualisation of the ductal tree will be repeated when the light source is switched to NBI. Possible findings during the assessment of the ductal tree are normal duct morphology, polypoid lesions, ductitis, epithelial lesions/damage, etc. When possible, the new biopsy tool will be used to remove the lesion and subsequently used histopathologic examination. If the removal of the lesion is unsuccessful or incomplete, intraductal laser ablation will be performed as described in a previous study ${ }^{44}$. Laser ductoscopy will be performed by applying 1-3W with 100-1000ms pulsed exposure generator (figure 4. CE marked Revolix Junior 30; LISA Laser Products, Katlenburg, Germany) to a CE marked $\mathrm{OH}$ silica fiber (Tobrix, Waalre, The Netherlands) with a core diameter of $200 \mathrm{~mm}$ and an outer diameter of $375 \mathrm{~mm}$. 

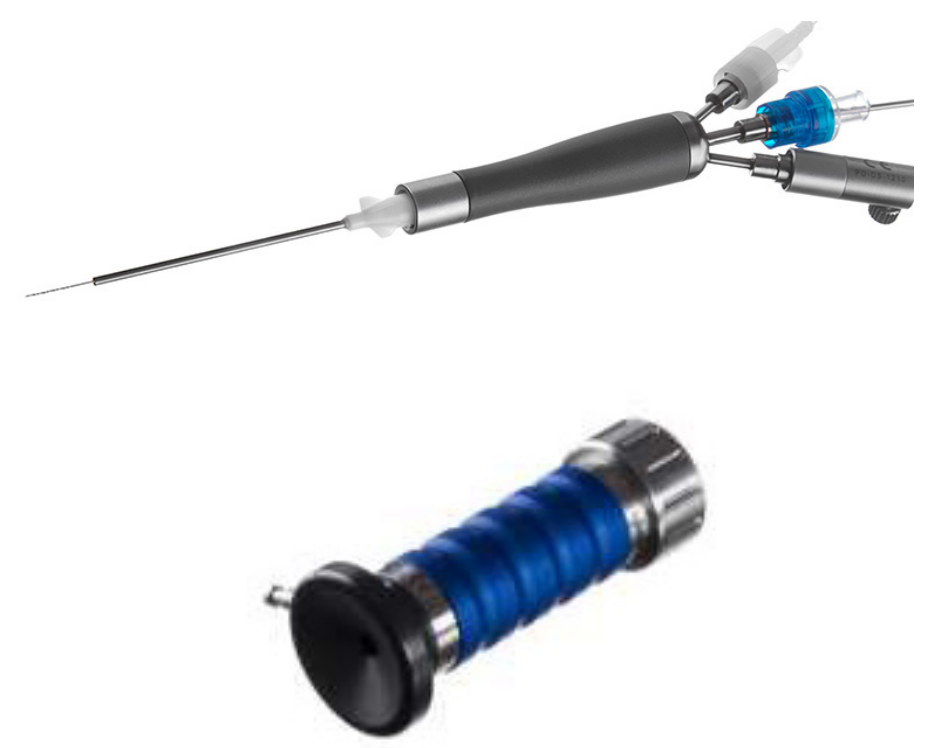

Figure 2. PD-DS-1084 (top) and Ocular Fix-Focus Blue (bottom).

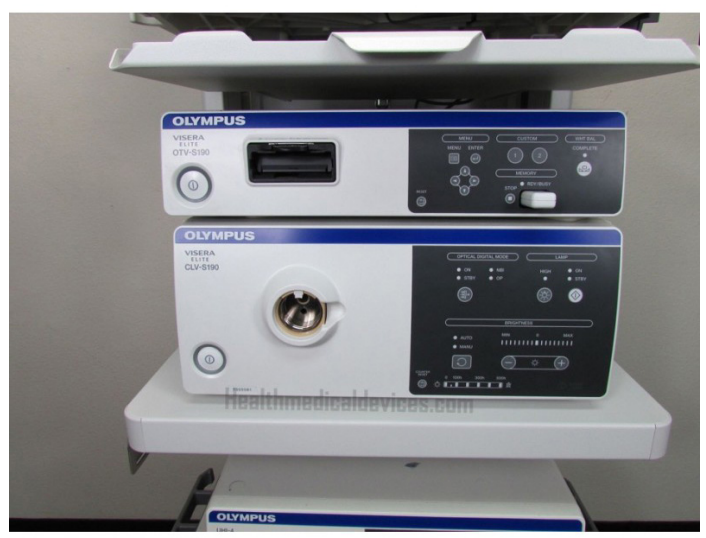

Figure 3. Olympus UM OTV-S190 video processor (top) and Olympus UM CLV-S190 light source (bottom). 


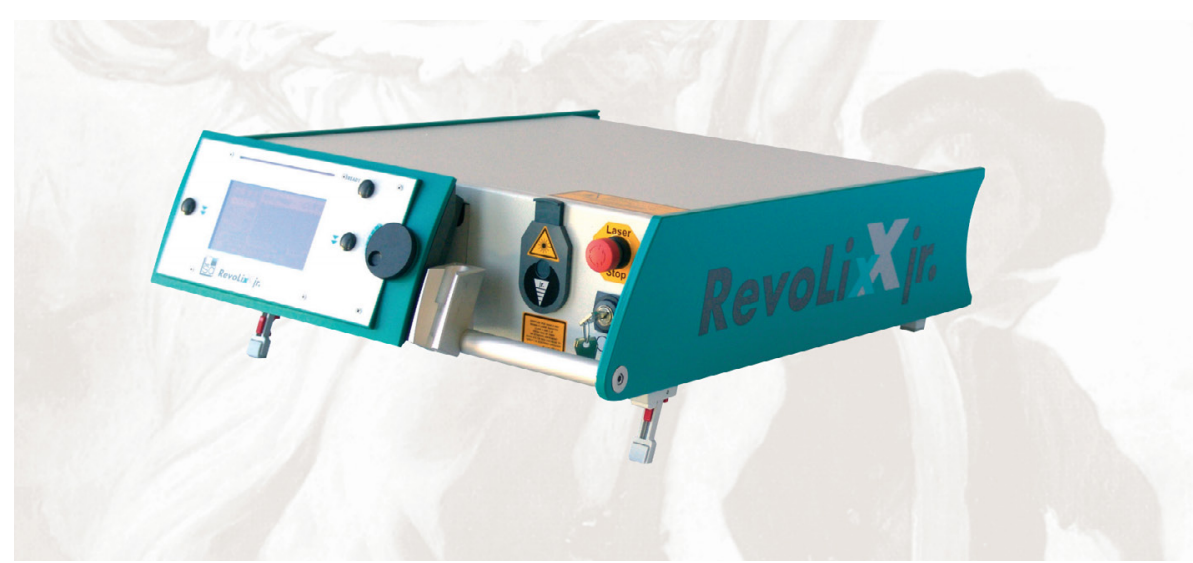

Figure 4. Lisa Laser Revolix jr 30.

\section{Intervention arm B}

This study will be performed in a very similar fashion as described for arm A. The only difference is that patients in arm B will be under general anaesthesia (with no local anaesthesia) and the entire procedure will be directly followed by preventive or therapeutic breast surgery. 


\section{Discussion}

In this trial, patients of both arms undergo white light ductoscopy, NBI ductoscopy, intraductal biopsy and intraductal laser ablation. We defined enhanced ductoscopy as regular ductoscopy combined with NBI, improved biopsy tool and intraductal laser ablation.

Ductoscopy shows to have a sensitivity of $71.4 \%$ and specificity of $97.9 \%$ for the detection of breast cancer in patients with PND without radiological signs of malignancy. ${ }^{27}$ Additionally, ductoscopy detects (pre)cancerous lesions that were missed during regular imaging ${ }^{27,46,47}$. At the same time, MRI has sensitivity ranging from 46 to $86 \%$ and specificity from 76 to $98 \%$ in the same patient population. ${ }^{48-50}$ Nevertheless, this trial might show that enhanced ductoscopy might further increase the diagnostic performance (with NBI and/or intraductal biopsy). Additionally, enhanced ductoscopy could be used to minimally invasively monitor and pathologically determine whether high-risk women are developing (pre)cancerous lesions.

Ductoscopy has already shown to prevent unnecessary surgery in patients with PND without radiological suspicion for malignancy. ${ }^{27,51}$ Papillomas are the most common cause of PND and are difficult to completely remove. ${ }^{23,27}$ Therefore, this study expects that enhanced (intraductal biopsy tool and/or intraductal laser ablation) ductoscopy might improve the extraction of intraductal lesions thereby alleviating symptoms of PND.

Enhanced ductoscopy could be even more useful to prevent unnecessary surgery in patients by treating and histologically diagnosing PND without radiological signs of malignancy. Additionally, the enhanced ductoscopy could also be added as a screening tool for women with a high risk of developing breast cancer in order to better time the moment of mastectomy. 


\section{References}

1. Ferlay J, Colombet M, Soerjomataram I, et al. Cancer incidence and mortality patterns in Europe: Estimates for 40 countries and 25 major cancers in 2018. Eur J Cancer. 2018;103:356-387. doi:S0959-8049(18)30955-9 [pii]

2. Ford D, Easton DF, Stratton M, et al. Genetic heterogeneity and penetrance analysis of the BRCA1 and BRCA2 genes in breast cancer families. The Breast Cancer Linkage Consortium. Am J Hum Genet. 1998;62(3):676-689. doi:S0002-9297(07)63848-8 [pii]

3. Tung N, Battelli C, Allen B, et al. Frequency of mutations in individuals with breast cancer referred for BRCA1 and BRCA2 testing using next-generation sequencing with a 25-gene panel. Cancer. 2015;121(1):25-33 doi:10.1002/cncr.29010 [doi]

4. Robson ME, Bradbury AR, Arun B, et al. American Society of Clinical Oncology Policy Statement Update: Genetic and Genomic Testing for Cancer Susceptibility. J Clin Oncol. 2015;33(31):3660-3667. doi:10.1200/JCO.2015.63.0996 [doi]

5. Couch FJ, Shimelis H, Hu C, et al. Associations Between Cancer Predisposition Testing Panel Genes and Breast Cancer. JAMA Oncol. 2017;3(9):1190-1196. doi:10.1001/jamaoncol.2017.0424 [doi]

6. Ludwig KK, Neuner J, Butler A, Geurts JL, Kong AL. Risk reduction and survival benefit of prophylactic surgery in BRCA mutation carriers, a systematic review. Am J Surg. 2016;212(4):660-669. doi:S0002-9610(16)30348-8 [pii]

7. Osman F, Saleh F, Jackson TD, Corrigan MA, Cil T. Increased postoperative complications in bilateral mastectomy patients compared to unilateral mastectomy: an analysis of the NSQIP database. Ann Surg Oncol. 2013;20(10):3212-3217. doi:10.1245/s10434-013-3116-1 [doi]

8. Eisen A, Rebbeck TR, Wood WC, Weber BL. Prophylactic surgery in women with a hereditary predisposition to breast and ovarian cancer. J Clin Oncol. 2000;18(9):1980-1995. doi:10.1200/JCO.2000.18.9.1980 [doi]

9. Hussain AN, Policarpio C, Vincent MT. Evaluating nipple discharge. Obstet Gynecol Surv. 2006;61(4):278283. doi:0006254-200604000-00025 [pii]

10. Dixon JM, Mansel RE. ABC of breast diseases. Symptoms assessment and guidelines for referral. BMJ. 1994;309(6956):722-726.

11. Seltzer MH. Breast complaints, biopsies, and cancer correlated with age in 10,000 consecutive new surgical referrals. Breast J. 2004;10(2):111-117. doi:21284 [pii]

12. Santen RJ, Mansel R. Benign breast disorders. N Engl J Med. 2005;353(3):275-285. doi:353/3/275 [pii]

13. King TA, Carter KM, Bolton JS, Fuhrman GM. A simple approach to nipple discharge. Am Surg. 2000;66(10):960-966.

14. Alcock C, Layer GT. Predicting occult malignancy in nipple discharge. ANZ J Surg. 2010;80(9):646-649. doi:10.1111/j.1445-2197.2010.05270.x [doi]

15. Albrecht C, Thele F, Grunwald S, et al. Nipple discharge: role of ductoscopy in comparison with standard diagnostic tests. Onkologie. 2013;36(1-2):12-16. doi:10.1159/000346639 [doi]

16. Yamamoto D, Shoji T, Kawanishi H, et al. A utility of ductography and fiberoptic ductoscopy for patients with nipple discharge. Breast Cancer Res Treat. 2001;70(2):103-108.

17. Yamamoto D, Ueda S, Senzaki H, et al. New diagnostic approach to intracystic lesions of the breast by fiberoptic ductoscopy. Anticancer Res. 2001;21(6A):4113-4116.

18. Okazaki A, Okazaki M, Asaishi K, et al. Fiberoptic ductoscopy of the breast: a new diagnostic procedure for nipple discharge. Jpn J Clin Oncol. 1991;21(3):188-193. 
19. Matsunaga T, Ohta D, Misaka T, et al. Mammary ductoscopy for diagnosis and treatment of intraductal lesions of the breast. Breast Cancer. 2001;8(3):213-221.

20. Grunwald S, Heyer H, Paepke S, et al. Diagnostic value of ductoscopy in the diagnosis of nipple discharge and intraductal proliferations in comparison to standard methods. Onkologie. 2007;30(5):243-248 doi:100848 [pii]

21. Han Y, Li J, Han S, Jia S, Zhang Y, Zhang W. Diagnostic value of endoscopic appearance during ductoscopy in patients with pathological nipple discharge. BMC Cancer. 2017;17(1):300-303. doi:10.1186/s12885017-3288-3 [doi]

22. Kamali S, Bender O, Kamali GH, Aydin MT, Karatepe O, Yuney E. Diagnostic and therapeutic value of ductoscopy in nipple discharge and intraductal proliferations compared with standard methods. Breast Cancer. 2014;21(2):154-161. doi:10.1007/s12282-012-0377-7 [doi]

23. Waaijer L, van Diest PJ, Verkooijen HM, et al. Interventional ductoscopy in patients with pathological nipple discharge. Br J Surg. 2015;102(13):1639-1648. doi:10.1002/bjs.9950 [doi]

24. Jacobs VR, Kiechle M, Plattner B, Fischer T, Paepke S. Breast ductoscopy with a 0.55-mm miniendoscope for direct visualization of intraductal lesions. J Minim Invasive Gynecol. 2005;12(4):359-364. doi:S1553-4650(05)00274-8 [pii]

25. Waaijer L, Simons JM, Borel Rinkes IH, van Diest PJ, Verkooijen HM, Witkamp AJ. Systematic review and meta-analysis of the diagnostic accuracy of ductoscopy in patients with pathological nipple discharge. $\mathrm{Br} \mathrm{J}$ Surg. 2016;103(6):632-643. doi:10.1002/bjs.10125 [doi]

26. Makita M, Akiyama F, Gomi N, Iwase T. Mammary ductoscopy and watchful follow-up substitute microdochectomy in patients with bloody nipple discharge. Breast Cancer. 2016;23(2):242-251. doi:10.1007/s12282-014-0561-z [doi]

27. Filipe MD, Waaijer L, van der Pol CC, van Diest P, Witkamp AJ. Interventional ductoscopy as an alternative for major duct excision or microdochectomy in women suffering pathological nipple discharge: a single centre experience. Clin Breast Cancer. Published online January 27, 2020. doi:10.1016/j.clbc.2019.12.008

28. Jacobs VR, Paepke S, Schaaf H, Weber BC, Kiechle-Bahat M. Autofluorescence ductoscopy: a new imaging technique for intraductal breast endoscopy. Clin Breast Cancer. 2007;7(8):619-623. doi:S1526-8209(11)70750-9 [pii]

29. Douplik A, Leong WL, Easson AM, Done S, Netchev G, Wilson BC. Feasibility study of autofluorescence mammary ductoscopy. J Biomed Opt. 2009;14(4):44036. doi:10.1117/1.3210773 [doi]

30. Gono K, Obi T, Yamaguchi M, et al. Appearance of enhanced tissue features in narrow-band endoscopic imaging. J Biomed Opt. 2004;9(3):568-577. doi:10.1117/1.1695563 [doi]

31. Singh R, Mei SC, Sethi S. Advanced endoscopic imaging in Barrett's oesophagus: a review on current practice. World J Gastroenterol. 2011;17(38):4271-4276. doi:10.3748/wjg.v17.i38.4271 [doi]

32. Tanaka S, Sano Y. Aim to unify the narrow band imaging (NBI) magnifying classification for colorectal tumors: current status in Japan from a summary of the consensus symposium in the 79th Annual Meeting of the Japan Gastroenterological Endoscopy Society. Dig Endosc. 2011;23 Suppl 1:131-139. doi:10.1111/ j.1443-1661.2011.01106.x [doi]

33. Efthymiou M, Taylor AC, Kamm MA. Cancer surveillance strategies in ulcerative colitis: the need for modernization. Inflamm Bowel Dis. 2011;17(8):1800-1813. doi:10.1002/ibd.21540 [doi]

34. Jichlinski P, Lovisa B. High magnification cystoscopy in the primary diagnosis of bladder tumors. Curr Opin Urol. 2011;21(5):398-402. doi:10.1097/MOU.0b013e32834956ad [doi] 
35. Boese A, Sivankutty AK, Illanes A, Friebe M. Intravascular endoscopy improvement through narrow-band imaging. Int J Comput Assist Radiol Surg. 2017;12(11):2015-2021. doi:10.1007/s11548-017-1579-4 [doi]

36. Ishida $Y$, Okabe $Y$, Yasumoto $\mathrm{M}$, et al. Ex vivo magnifying endoscopic observation of bile duct mucosa using narrowband imaging. J Hepatobiliary Pancreat Sci. 2018;25(10):433-439. doi:10.1002/jhbp.581 [doi]

37. Ruiz A, Almenar S, Cerda M, Hidalgo JJ, Puchades A, Llombart-Bosch A. Ductal carcinoma in situ of the breast: a comparative analysis of histology, nuclear area, ploidy, and neovascularization provides differentiation between low- and high-grade tumors. Breast J. 2002;8(3):139-144. doi:tbj08303 [pii]

38. Santamaria G, Velasco M, Farrus B, Caparros FX, Fernandez PL. Dynamic contrast-enhanced MRI reveals the extent and the microvascular pattern of breast ductal carcinoma in situ. Breast J. 2013;19(4):402-410. doi:10.1111/tbj.12135 [doi]

39. Gadre SA, Perkins GH, Sahin AA, Sneige N, Deavers MT, Middleton LP. Neovascularization in mucinous ductal carcinoma in situ suggests an alternative pathway for invasion. Histopathology. 2008;53(5):545-553. doi:10.1111/j.1365-2559.2008.03152.x [doi]

40. Li L, Wang K, Sun X, et al. Parameters of dynamic contrast-enhanced MRI as imaging markers for angiogenesis and proliferation in human breast cancer. Med Sci Monit. 2015;21:376-382. doi:10.12659/ MSM.892534 [doi]

41. Fox SB, Generali DG, Harris AL. Breast tumour angiogenesis. Breast Cancer Res. 2007;9(6):216. doi:10.1186/bcr1796 [doi]

42. Netsch C, Engbert A, Bach T, Gross AJ. Long-term outcome following Thulium VapoEnucleation of the prostate. World J Urol. 2014;32(6):1551-1558. doi:10.1007/s00345-014-1260-2 [doi]

43. Allam JP, Novak N. The pathophysiology of atopic eczema. Clin Exp Dermatol. 2006;31(1):89-93. doi:CED1980 [pii]

44. de Boorder T, Waaijer L, van Diest PJ, Witkamp AJ. Ex vivo feasibility study of endoscopic intraductal laser ablation of the breast. Lasers Surg Med. 2018;50(2):137-142. doi:10.1002/lsm.22745

45. Waaijer L, van Diest P, van der Pol CC, Verolme B, Hennink A, Witkamp AJ. Ductoscopy for pathologic nipple discharge. Ned Tijdschr Geneeskd. 2013;157(29):A6358.

46. Bender O, Balci FL, Yuney E, Akbulut H. Scarless endoscopic papillomectomy of the breast. Onkologie. 2009;32(3):94-98. doi:10.1159/000195694 [doi]

47. Gui G, Agusti A, Twelves D, et al. INTEND II randomized clinical trial of intraoperative duct endoscopy in pathological nipple discharge. Br J Surg. 2018;105(12):1583-1590. doi:10.1002/bjs.10990 [doi]

48. van Gelder L, Bisschops RH, Menke-Pluymers MB, Westenend PJ, Plaisier PW. Magnetic resonance imaging in patients with unilateral bloody nipple discharge; useful when conventional diagnostics are negative? World J Surg. 2015;39(1):184-186. doi:10.1007/s00268-014-2701-1 [doi]

49. Zacharioudakis K, Kontoulis T, Vella JX, et al. Can we see what is invisible? The role of MRI in the evaluation and management of patients with pathological nipple discharge. Breast Cancer Res Treat. 2019;178(1):115120. doi:10.1007/s10549-019-05321-w [doi]

50. Bahl M, Gadd MA, Lehman CD. JOURNAL CLUB: Diagnostic Utility of MRI After Negative or Inconclusive Mammography for the Evaluation of Pathologic Nipple Discharge. AJRAmerican J Roentgenol. 2017;209(6):1404-1410. doi:10.2214/AJR.17.18139 [doi]

51. Chang YK, Chen CTH, Wang M, et al. Could ductoscopy alleviate the need of microdochectomy in pathological nipple discharge? Breast Cancer. Published online February 1, 2020. doi:10.1007/s12282-02001051-w 


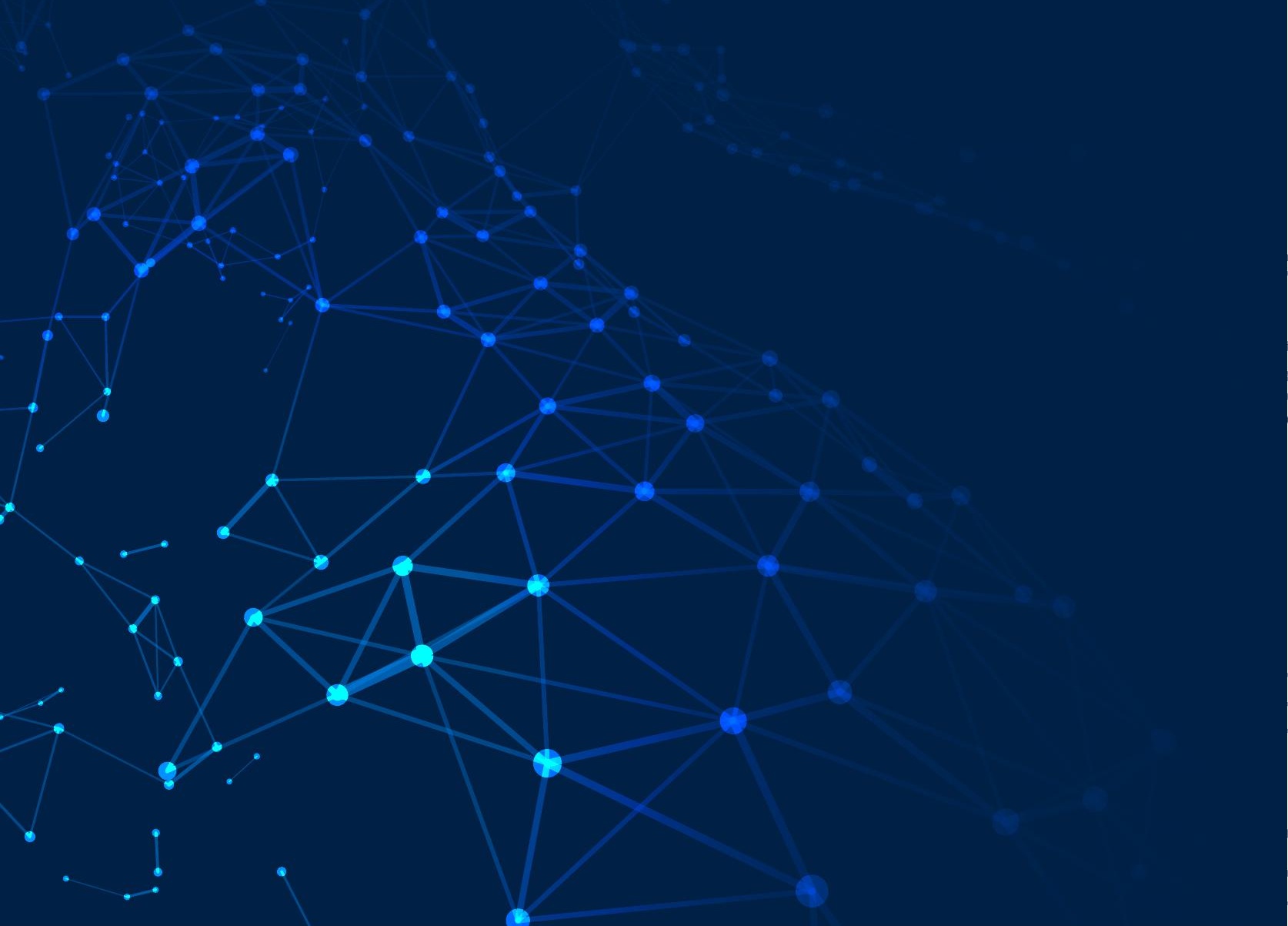





\section{Chapter 6}

Meta-analysis and cost-effectiveness of ductoscopy, duct excision surgery and MRI for the diagnosis and treatment of patients with pathological nipple discharge

S.I.S. Patuleia

M.R. Vriens

P.J. van Diest

A.J. Witkamp 


\begin{abstract}
Introduction: Pathological nipple discharge (PND) is a common breast-related complaint for referral to a surgical breast clinic because of its association with breast cancer. The aim of this meta-analysis was to compare the diagnostic efficacy of magnetic resonance imaging (MRI) and ductoscopy in patients with PND. Additionally, we determined the most cost-efficient strategy for the treatment of PND and the detection of breast cancer in PND patient without radiological suspicion for malignancy.
\end{abstract}

Materials and Methods: PubMed and EMBASE were searched to collect the relevant literature from the inception of both diagnostic methods until January $27^{\text {th }} 2020$. The search yielded 815 original citations, of which 10 studies with 894 patients were finally included for analysis. Costs of ductoscopy, MRI and duct excision surgery were obtained from the UMC Utrecht as established in the year 2019. These costs included: medical personnel, overhead costs, material costs and sterilisation costs.

Results: The meta-analysis showed no significant difference in sensitivity between ductoscopy (44\%) and MRI (76\%) for the detection of malignancy in patients with PND. However, ductoscopy (98\%) had a statistically significantly higher specificity than MRI (84\%). Individual costs were $€ 1,401.33$, €822.13 and $€ 6,494.27$ for ductoscopy, MRI and duct excision surgery, respectively. Full diagnostic strategy involving ductoscopy was on average $€ 1,670.97$, while with MRI it was $€ 2,070.27$.

Conclusion: Patients undergoing MRI are more often (false) positive which more often leads to duct excision surgery referrals compared to ductoscopy. This makes ductoscopy significantly more cost-effective compared MRI in patients with PND without radiological suspicion for malignancy. 


\section{Introduction}

Pathological nipple discharge (PND) is defined as unilateral, spontaneous and bloody or serous discharge, usually arising from a single duct orifice of the nipple. After pain and palpable lumps, PND is the third most common breast-related complaint ${ }^{1}$ and it accounts for 3-5\% of surgical breast clinic referrals. ${ }^{2-5}$ Even though it is considered a red-flag symptom for breast cancer, the most common causes of PND are benign, namely ductal ectasia and intraductal papillomas. ${ }^{6,7}$

Traditionally, patients suffering PND are offered major duct excision surgery to rule out malignancy, ${ }^{6,8,9}$ which occurs in only 5-8\%.5,10,11 This means that around $92-95 \%$ of these operations are performed for non-malignant causes. However, although invasive, the advantage of major duct excision is that it can also be helpful to treat PND itself.

Magnetic resonance imaging (MRI) has more recently shown to be a sensitive tool for the detection of malignancy in patients with PND. However, MRI has some shortcomings, namely in the detection of small lesions and in differentiating benign from malignant masses. 9,12 Therefore, the value of MRI is limited in patients with PND and core needle biopsy or surgical excision is still necessary when MRI shows a suspicious lesion. ${ }^{13,14}$ This not only leads to a longer diagnostic path but is also accompanied by accumulation of costs.

Ductoscopy is a minimally invasive micro-endoscopic technique providing real-time visualization of the milk ducts of the breast. This procedure is performed under local anaesthesia at the outpatient clinic and is currently used as a diagnostic tool in the workup of women suffering from PND ${ }^{15-22}$. Ductoscopy has been shown to be a useful tool in finding intraductal lesions causing PND (benign and malignant). ${ }^{23-25} \mathrm{Next}$ to its diagnostic role, ductoscopy can potentially treat the actual cause of PND as well by mechanical removal ${ }^{26}$ or laser ablation ${ }^{27}$ of intraductal lesions like papillomas. Therefore, ductoscopy has the ability to replace invasive surgical procedures in patients suffering from PND.

Besides their difference in diagnostic and therapeutic capabilities, major duct excision, MRI and ductoscopy also differ in costs. For example, although effective in the actual treatment of PND, the costs of major duct excision exceed those of MRI and ductoscopy together. So, better selection of patients that actually will benefit from duct excision is crucial to safe costs and to save women from the undesirable side effects of surgery.

As the above shows, there is a need to establish the most cost-effective work-up for women presenting with PND. Therefore, the aim of our study was to compare the diagnostic performance for detecting breast cancer of ductoscopy and MRI in patients with PND in order to better select who is eligible for surgery. Additionally, we performed a costeffectiveness analysis (CEA) for the diagnostic performance for detecting breast cancer of ductoscopy and MRI, followed by a CEA for the treatment of PND comparing major duct excision and ductoscopy. 


\section{Materials and methods}

\section{Meta-analysis}

The systematic literature search on the diagnostic performance of ductoscopy and MRI was performed according to the Preferred Reporting Items for Systematic Reviews and Meta-Analyses (PRISMA) guidelines for meta-analysis. ${ }^{28}$ The PubMed and Embase databases were systematically searched for studies published until January 2020. The search strategy was performed on synonyms and medical subject heading (MESH) terms of pathological nipple discharge and the index tests (MRI and ductoscopy). Only articles that evaluated MRI and/or ductoscopy, reported original data and were written in English were selected. Full syntaxes are shown in Appendix 1. After removal of duplicates, two authors (MF, SP) independently screened articles by title, abstract and full text. Any disagreement was solved through discussion to reach a consensus.

\section{Selection of studies}

Title/abstract screening was performed after removal of duplicates. Full texts were retrieved for studies that evaluated MRI and/or ductoscopy, reported original data and were written in English.

1. Participants: patients with PND without history of breast cancer or radiological suspicion of breast cancer.

2. Intervention: MRI and/or ductoscopy.

3. Comparator: all patients must have had definitive diagnosis of malignancy by the means of biopsy or histopathological analysis after surgery.

4. Outcome: diagnostic performance of ductoscopy and MRI for the detection of (pre)cancerous lesions.

5. Study characteristics: all studies accepted for publication written in English.

Studies were excluded from systematic review owing to the following reasons:

1. Not possible to determine sensitivity and specificity from the studies by means of true positive, true negative, false positive and true negative.

2. Studies in which none of the patients had histopathological confirmation of malignancy.

3. Case report, review and conference abstracts. 


\section{Risk of bias}

The QUADAS-2 Tool was used to evaluate the quality of each eligible study. ${ }^{29}$ The entire scale constituted four domains for the risk of bias: patient selection, index test, reference standard and flow and timing. Additionally, there were three domains for applicability concerns: patient selection, index test and reference standard. Each domain could be judged as any of the three levels, low risk, intermediate/unclear risk, or high risk of bias. Additionally, funnel plots and Egger's test were performed in order to see whether there was publication or small sampling bias. ${ }^{30}$

\section{Classifications}

MRI scans were classified according to the Breast Imaging Reporting and Data System (BI-RADS) reporting system. ${ }^{31}$ BI-RADS I-III was considered benign and BI-RADS IV to VI were considered suspicious for malignancy or malignant.

\section{Cost-effectiveness analysis model}

Firstly, a CEA model was developed to capture the costs and effectiveness of ductoscopy, MRI and duct excision surgery. In this model, surgery was performed if ductoscopy or MRI was suspicious for breast cancer. Model outputs were represented in terms of effects of diagnostic success for the detection of breast cancer in patients with PND without suspicion for malignancy on ultrasound or mammography. A random sample of 10,000 patients per diagnostic method was generated with an incidence of $5 \%$ based on literature. ${ }^{10,11}$ Analysis was performed with 100 bootstraps.

Secondly, another CEA model was developed to determine the costs and effectiveness of ductoscopy in treating PND. Data were obtained from our previous clinical study. ${ }^{26}$ Model outputs were represented in terms of effect of therapeutic success after ductoscopy and/or surgery in patients with PND without suspicion for malignancy on ultrasound or mammography.

A univariate sensitivity analysis was performed using as minimum and maximum values the lower and upper limits of the $95 \%$ confidence intervals for the sensitivity of ductoscopy and MRI. In addition, univariate sensitivity analysis was also performed with the different rates of successful ductoscopy according to the literature.

\section{Cost calculation}

Costs of ductoscopy comprised actual staff and equipment costs since ductoscopy is currently not (yet) covered by medical insurance in The Netherlands. The staff costs covered the surgeon performing the ductoscopy, two nurses (one scrub nurse and one circulating 
nurse) and overhead costs. Equipment costs consisted of ductoscopy materials (hardware and reusables), sterilisation costs and overhead costs. The costs were incorporated in a decision model using probabilities of events and unit costs of ductoscopy and MRI ${ }^{32}$. The total costs of surgery were estimated based on average overall hospital costs, including surgical or nonsurgical charges of the UMC Utrecht. All costs are presented in Euros (€), according to the price quotes of 2019.

\section{Statistical analysis}

Firstly, sensitivity and specificity were calculated for ductoscopy and MRI with the $95 \%$ confidence interval (CI). After this, pooled estimates of sensitivity and specificity were calculated for ductoscopy and MRI using fixed-effects. Heterogeneity among studies was quantified by the I-square and tested using Cochran's-Chi-square tests. Subsequently, the chance of a positive test (for MRI and ductoscopy), positive predictive value (PPV) and negative predictive value (NPV) were calculated from the pooled sensitivity, specificity and the prevalence of breast cancer using the following formulas:

$$
\begin{aligned}
& \text { positive test }=\text { prevalence } * \text { sensitivity }+(1-\text { prevalence }) *(1-\text { specificity }) \\
& P P V=\text { prevalence } * \frac{\text { sensitivity }}{\text { prevalence } * \text { sensitivity }+(1-\text { prevalence }) *(1-\text { specificity })} \\
& N P V=(1-\text { prevalence }) * \frac{\text { specificity }}{\text { prevalence } *(1-\text { sensitivity })+(1-\text { prevalence }) * \text { specificity }} \\
& D A=\text { sensitivity } * \frac{\text { specificity }}{(1-\text { sensitivity }) *(1-\text { specificity })}
\end{aligned}
$$

Decision trees were modelled using TreeAge Pro V.2015 (TreeAge Software, Williamstown, Massachusetts, USA). All calculations were performed using RStudio 1.2.5001 (with R version: x64 3.6.3). Additionally, statistical packages meta, mada, metaphor and mvmeta were used for all computations and visualisations of the meta-analysis. Cost-effectiveness computations and visualisations were performed using ICEinfer package. Finally, other visualisation of plots was done using the ggplot 2 package. 


\section{Results}

\section{Meta-analysis}

A total of 815 citations of articles in English language were identified by the search and, after removing duplicates and screening on relevance, 73 potentially eligible articles were retrieved in full text (figure 1). Overall, 894 patients in 10 studies with PND underwent ductoscopy, MRI and/or duct excision surgery. Table 1 shows the details of the studies used in the analysis.

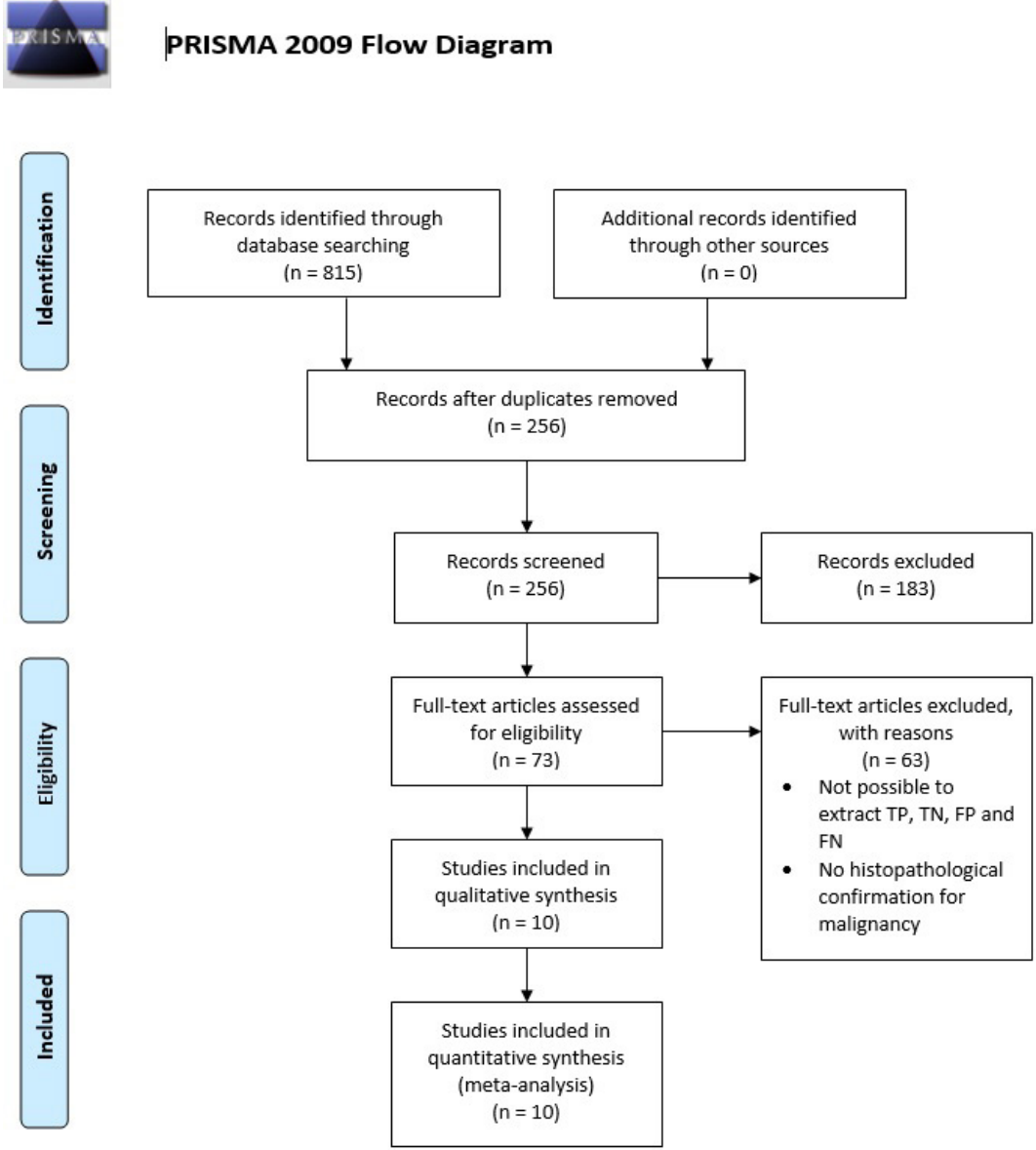

Figure 1. Flow chart showing literature search and study selection with 10 relevant studies ultimately enrolled in the meta-analysis. $\mathrm{N}=$ Number, $\mathrm{TP}=$ True positive, $\mathrm{TN}=$ True negative, $\mathrm{FN}=$ False negative and $\mathrm{FP}=\mathrm{False}$ positive. 
Table 1. Baseline characteristics of studies on diagnostic modalities in patients with pathologic nipple discharge without radiological signs of malignancy.

\begin{tabular}{llllll}
\hline Author & Year & Country & Standard method & N & $\begin{array}{l}\text { Diagnostic } \\
\text { modalities }\end{array}$ \\
\hline Morrogh et al. ${ }^{33}$ & 2007 & USA & histopathological diagnosis & 33 & MRI \\
Denewer et al. ${ }^{34}$ & 2008 & Egypt & histopathological diagnosis & 53 & Ductoscopy \\
Bender et al. ${ }^{35}$ & 2009 & Turkey & histopathological diagnosis & 102 & Ductoscopy \\
Vaughan et al. ${ }^{36}$ & 2009 & USA & histopathological diagnosis & 89 & Ductoscopy \\
van Gelder et al. ${ }^{12}$ & 2015 & Netherlands & histopathological diagnosis & 107 & MRI \\
Sanders et al. ${ }^{9}$ & 2016 & USA & histopathological diagnosis & 85 & MRI \\
Bahl et al. ${ }^{37}$ & 2017 & USA & histopathological diagnosis & 105 & MRI \\
Gui et al. ${ }^{38}$ & 2018 & UK & histopathological diagnosis & 32 & Ductoscopy \\
Zacharioudakis et al. ${ }^{39}$ & 2019 & UK & histopathological diagnosis & 82 & MRI \\
Filipe et al. ${ }^{26}$ & 2020 & Netherlands & histopathological diagnosis & 206 & Ductoscopy \\
\hline
\end{tabular}

UK = united Kingdom; USA = United States of America; $\mathrm{N}$ = total number of patients; 

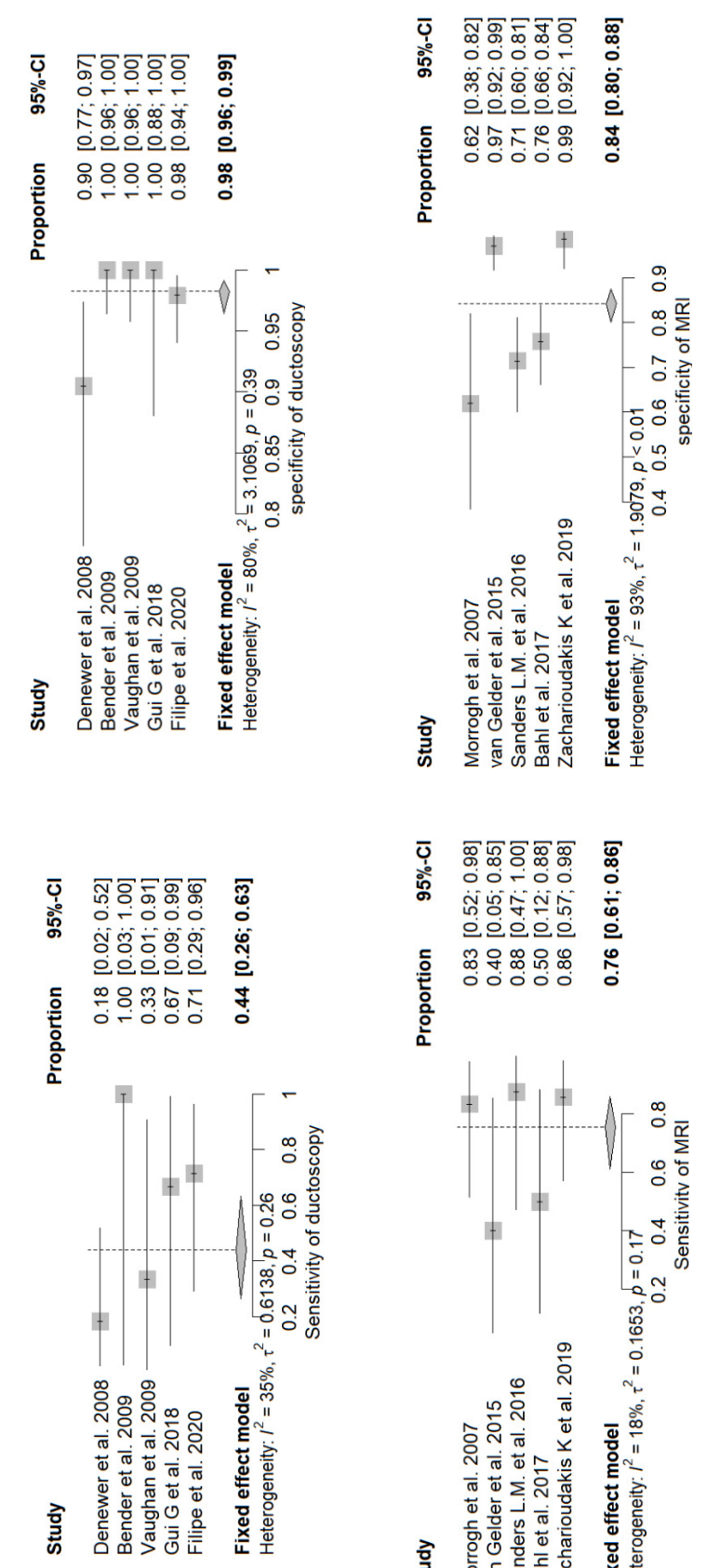

\section{(5)}

কי্চ

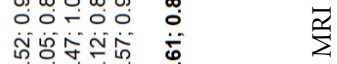

드으은

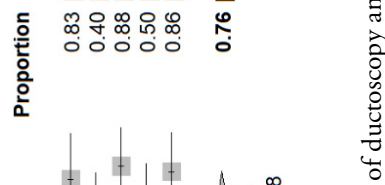

各

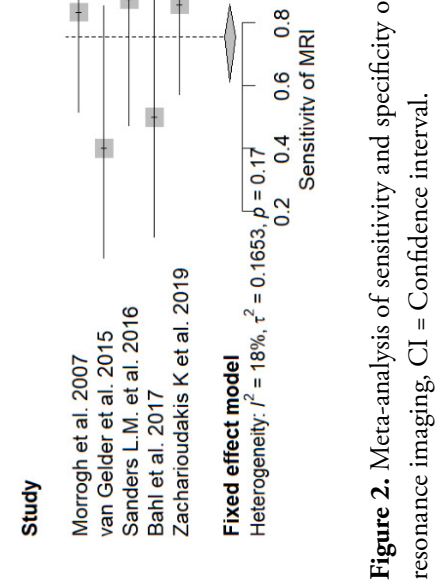


Figure 2 shows the diagnostic performance of ductoscopy and MRI. Ductoscopy had a pooled sensitivity of $44 \%$ (95\% CI of 22-66\%) for detection of breast cancer and a specificity of $98 \%$ (95\% CI of $96-99 \%)$ for the detection of malignancy. Sensitivity and specificity of MRI were 76\% (95\% CI of 71-86\%) and $84 \%$ (95\% 80-88\%), respectively. The prevalence of malignancy in patients with PND without radiological suspicion for malignancy was around $5 \% .{ }^{10,11}$ Based on this prevalence, estimated PPV and NPV were $53.7 \%$ and $97.1 \%$ for ductoscopy, respectively. MRI had an estimated PPV of $20 \%$ and a NPV of $98.5 \%$.

The result of the QUADAS-2 tool revealed that all the included studies were of sufficient quality. This was for both risks of bias domains and applicability domains (Supplementary figure 1). Additionally, this study showed symmetry of the effect, indicating no evidence for a small sample effect or publication bias in the subgroup analysis (Supplementary figure 2). P-values for Egger's test for sensitivity and specificity were 0.0504 and 0.755 , respectively.

\section{Cost analysis}

The input model (Table 2) was based on the data from the meta-analysis of this study, as well as other references and findings of the financial departments of the UMC Utrecht. The average costs of a ductoscopy procedure, a major duct excision operation and a breast MRI at the UMC Utrecht in the year 2019 were $€ 1,401.33$, $€ 6,494.27$ and $€ 822.13$, respectively. 
Table 2. Model inputs: clinical and cost parameters $(2019, €)$ of ductoscopy and MRI in patients with PND without radiological signs of malignancy.

\begin{tabular}{lll}
\hline Parameters & Values & Source \\
\hline Prevalence of breast cancer & $5 \%$ & 10,11 \\
Ductoscopy parameters & & \\
$\quad$ Sensitivity & $44 \%(95 \%$ CI 22-66\%) & Study data \\
Specificity & $98 \%(95 \%$ CI $96-99 \%)$ & Study data \\
Successful ductoscopy rate & $70 \%(95 \%$ CI 70-95\%) & Study data \\
PND stopped after successful ductoscopy & $60.3 \%$ & 26 \\
PND stopped after unsuccessful ductoscopy & $29.7 \%$ & 26 \\
Costs of ductoscopy & $€ 1,401.33$ & Study data \\
MRI parameters & & \\
Sensitivity & $76 \%(95 \%$ CI 71-86\%) & Study data \\
Specificity & $84 \%(95 \%$ CI $80-88 \%)$ & Study data \\
Costs of MRI & $€ 822.13$ & Study data \\
Surgery parameters & & \\
PND stopped after surgery & $100 \%$ & Expert opinion \\
Costs of surgery & $€ 6,494.27$ & Study data \\
\hline
\end{tabular}

$\mathrm{PND}=$ pathological nipple discharge, $\mathrm{MRI}=$ Magnetic resonance imaging, $\mathrm{CI}=$ Confidence interval

\section{Diagnostic cost-effectiveness analysis comparing ductoscopy to MRI}

Based on the diagnostic performance (sensitivity, specificity, PPV and NPV) calculated earlier, Figure 3 shows the CEA of the diagnostic performance of ductoscopy, duct excision surgery and MRI for the detection of cancer in patients with PND with negative conventional radiological findings.

The chance of positive findings at ductoscopy (including unsuccessful ductoscopy procedures) was $4.1 \%$, of which $53.7 \%$ were true positive. Consequently, the chance of negative findings at ductoscopy was $95.9 \%$ of which $97.1 \%$ was true negative. Hence, based on diagnostic performances and costs, the average cost of ductoscopy to diagnose (pre)cancerous lesions, and subsequent surgery when positive, would be $€ 1,670.97$. The diagnostic accuracy of ductoscopy was $95.3 \%$.

MRI was positive in $19.0 \%$ of the cases, of which $20.0 \%$ were true positive. Therefore, the chance of a negative MRI was $81.0 \%$ of which $98.5 \%$ was true negative. Furthermore, the average estimated cost using MRI to diagnose (pre)cancerous lesions, and subsequent surgery when positive, in PND patients would be $€ 2,070.27$. This based on the fact that $19 \%$ of patients with PND without radiological suspicion for malignancy are estimated to 
have a positive MRI and therefore referred for surgery. This results in a sum of the costs of MRI (€822.13) and the in $19 \%$ of the cases surgery (€6,494.27). The diagnostic accuracy of MRI was $83.6 \%$. Exact calculations can be found in supplementary figure 3 . The current study showed that ductoscopy was more cost-effective for the detection of malignancy in patient with PND compared to MRI, regardless of the margin of error of the sensitivity (95\% CI). Sensitivity analysis determining the different cost-effectiveness based on the $95 \%$ CI sensitivity of ductoscopy and MRI can be found in supplementary table 1 .

\section{Therapeutic cost-effectiveness analysis comparing ductoscopy to surgery}

In our previous study 215 patients underwent a ductoscopy procedure. The therapeutic success rate was defined as total relief of PND for at least three months (median follow-up was 14.1 months), regardless of the findings during the ductoscopy itself. The technical success rate of ductoscopy itself was $70.2 \%$ (i.e. the procedure could be fully accomplished and sufficient inspection of the ductal tree was possible). A total of 60 patients $(27.9 \%)$ were operated, for different reasons, in addition to ductoscopy; ductoscopy itself technically failed $(\mathrm{N}=24)$, suspicious findings $(\mathrm{N}=8)$ or the PND did not stop $(\mathrm{N}=42)$. In $60.3 \%$ of the technically successful ductoscopy procedures (i.e. inspection of the ductal tree was possible) the PND stopped, of which $7.7 \%$ were subsequently operated due to suspicious findings. Consequently, in $39.7 \%$ of technically successful ductoscopy procedures the PND did not stop of which $48.3 \%$ underwent surgery. ${ }^{26}$

Thereby, the effectivity of ductoscopy was $51.2 \%$ (percentage of patients that no longer suffered PND) and the average total cost of a patient with PND undergoing a therapeutic strategy with ductoscopy was $€ 3,208.89$. This cost is based on the sum of the cost for ductoscopy $(€ 1,401.33)$ and the fact that $48.3 \%$ of patients that underwent ductoscopy also underwent subsequent duct excision surgery $(€ 6,494.27)$. Exact calculations can be found in the supplementary figure 4 . The current study showed that ductoscopy was more cost-effective for the treatment of PND compared to duct excision surgery, regardless cannulation rates reported by the literature. Sensitivity analysis determining the different cost-effectiveness based on the cannulation of ductoscopy according to the literature can be found in supplementary table 2 . 


\section{Discussion}

In this study, a meta-analysis was performed on the diagnostic performances of ductoscopy and MRI for the detection of (pre)cancerous lesion in patients with PND without radiological suspicion for malignancy. This study also shows the results of a CEA comparing ductoscopy to MRI in this group of patients for the detection of malignancy and also their capability to select patients for major duct excision. Finally, we performed a CEA comparing ductoscopy to major duct excision for the therapeutic effect in resolving PND in these patients.

For the meta-analysis, 10 studies were finally selected, which together included a total of 894 patients suffering from PND. We compared ductoscopy to MRI in terms of sensitivity and specificity. Pooled sensitivity and specificity of ductoscopy were $44 \%$ and $98 \%$, respectively. MRI showed a pooled sensitivity of $76 \%$ and $84 \%$, respectively. There were no statistically significant differences in sensitivity, but specificity was statistically significantly higher for ductoscopy.

In recent years, MRI has been used more often for the detection of breast cancer in patients with PND. Based on our meta-analysis, MRI has a high sensitivity for the detection of breast cancer in this group. However, due to a relatively low specificity, histopathological assessment through surgery or biopsy remains necessary to determine whether the lesion is benign-or-not. ${ }^{13,14} \mathrm{MRI}$ is also increasingly used in cases of PND when mammography and ultrasound are negative. ${ }^{8,12,39,40}$ The current study clarifies that MRI has a higher sensitivity (although not significant) but shows at the same time a statistically significantly lower specificity in comparison to ductoscopy for the detection of breast cancer in patients with PND. Contrast enhanced MRI appears to be a promising imaging method for the detection of breast cancer in this group of patients. ${ }^{41-43}$ However, contrast enhanced MRI studies in PND patients are scarce and include only few patients for which reason they were not included in our meta-analysis. ${ }^{41-43}$

Over the last few decades, ductoscopy has been gaining ground for detection of lesions causing PND. ${ }^{34-36,38,44,45}$ The meta-analysis performed in this study shows that ductoscopy has a similar (not significant) sensitivity ( 44 vs $86 \%$ ) but a significantly higher (84 vs. $98 \%$ ) specificity in comparison with MRI. However, since the prevalence of (pre) cancerous lesions is only around 5\% in patients suffering PND without radiological signs of malignancy, specificity is a more useful tool to determine diagnostic performance. This is also reflected by the fact that our study shows that the diagnostic accuracy of ductoscopy $(95.3 \%)$ is significantly higher compared with MRI (83.6\%). Therefore, it is safe to conclude that ductoscopy seems to be a more useful tool to determine which patients are eligible for (duct excision) surgery. This is in line with previous studies that showed that ductoscopy successfully reduces the need for surgery in patients with PND. 26,46. Additionally, since intraductal extractions are nowadays possible with the basket 
extraction device and pilot studies with laser ablation have been done, ${ }^{26,47}$ ductoscopy also has a therapeutic potential in the treatment of PND itself, ${ }^{22}$ making it an even more attractive modality.

In our study we also show that ductoscopy is more beneficial (11.9\%) and less costly (€399.30) compared with MRI for the proper selection of PND patients for surgery. This can be explained by the specificity of MRI and ductoscopy. With a malignancy prevalence of $5 \%$, the significantly higher specificity of ductoscopy ensures that only $4.1 \%$ of ductoscopy procedures are positive (regardless whether it is true positive of false positive). This means that only $4.1 \%$ of patients will undergo surgery when ductoscopy is used. However, since specificity of MRI is significantly lower (84\%), despite the fact that sensitivity is higher, the chance of a positive MRI is $19 \%$. This means that $19 \%$ of patients with PND without radiological suspicion will undergo surgery. Consequently, when a MRI is performed, chances of a PND patient undergoing surgery is almost 5 times higher. Therefore, even though a single MRI is less costly than a ductoscopy (€822.13 vs. $€ 1,401.33$, respectively), this analysis shows that it would still be considerably less expensive to use ductoscopy as a strategy in determining the need for surgery in this patient population. Multivariate sensitivity analysis taking the uncertainties of the sensitivities for the detection of malignancy in patients with PND with negative conventional imaging of ductoscopy and MRI into account showed no significant changes to these conclusions.

As mentioned above, ductoscopy (unlike MRI) also has a potential therapeutic effect on the PND itself. This results in a further decrease in the number of major duct excisions needed. Based on the clinical data from our previous published study we showed that in over half $(51.7 \%)$ of patients undergoing (attempted) ductoscopy the PND actually stopped and only $27.9 \%$ of women suffering PND finally needs surgery after ductoscopy. ${ }^{26}$

Our study also has some limitations. First, the diagnostic section was modelled, based on pooled diagnostic performances of ductoscopy and MRI. However, there was an unexplained difference in the prevalence of malignancy between ductoscopy $(4.5 \%)$ and MRI (19\%) studies. This might explain the high heterogeneity for specificity in both ductoscopy and MRI. Since there is a consensus that the malignancy rate in patients with PND without radiological signs of malignancy is around $5 \%,{ }^{14,24}$ the current study parted from that premise and did not include prevalence in the sensitivity analysis. Second, in the included studies there are different definitions of a technically successful ductoscopy. This study defined successful ductoscopy as being able to visualise the ductal tree. However, other studies defined successful ductoscopy as being able to cannulate the ductal tree, regardless of being able to visualise-or-not. We performed a sensitivity analysis for this uncertainty but this did not change the conclusions. Thirdly, since most intraductal lesions causing PND are directly behind the nipple, biopsy is often not possible and surgery is recommended. ${ }^{48,49}$ For this reason costs of biopsy were not taken into account in this study. Fourthly, all costs were obtained from only one hospital, since the UMC Utrecht is the only hospital in The 
Netherlands performing ductoscopy. Although costs for medical procedures might differ from one hospital to another, it does not seem very likely that the ratio between these costs within one hospital will vary much. Therefore, the effect on our analysis is probably limited. At last, there are currently no studies describing the quality of life of patients with PND undergoing MRI, ductoscopy or duct excision surgery. For that reason, quality of adjusted life analysis was not used in this study. However, it is reasonable to assume that surgery has a greater negative impact on quality of life compared with MRI or ductoscopy and should therefore be avoided if possible.

To conclude, this study is the first to directly compare the diagnostic performance of ductoscopy and MRI in patients with PND without radiological signs of malignancy. This study shows that ductoscopy has a significantly higher diagnostic accuracy in this patient population. This makes ductoscopy less costly and a more effective diagnostic tool in comparison with MRI to determine which patients finally require surgery to rule out malignancy. Furthermore, this CEA showed that, while ductoscopy is not as effective in treating PND as duct excision surgery, it is much less costly. 


\section{References}

1. Hussain AN, Policarpio C, Vincent MT. Evaluating nipple discharge. Obstet Gynecol Surv. 2006;61(4):278-283. doi:0006254-200604000-00025 [pii]

2. Dixon JM, Mansel RE. ABC of breast diseases. Symptoms assessment and guidelines for referral. BMJ. 1994;309(6956):722-726.

3. Seltzer MH. Breast complaints, biopsies, and cancer correlated with age in 10,000 consecutive new surgical referrals. Breast J. 2004;10(2):111-117. doi:21284 [pii]

4. Santen RJ, Mansel R. Benign breast disorders. N Engl J Med. 2005;353(3):275-285. doi:353/3/275 [pii]

5. T.A. K, K.M. C, J.S. B, et al. A simple approach to nipple discharge. Am Surg. 2000;66(10):960-966. http://www.embase.com/search/results?subaction=viewrecord\&from=export\&id=L30777959.

6. C. A, G.T. L, Alcock C, Layer GT. Predicting occult malignancy in nipple discharge. ANZ J Surg. 2010;80(9):646-649. doi:10.1111/j.1445-2197.2010.05270.x [doi]

7. Albrecht C, Thele F, Grunwald S, et al. Nipple discharge: role of ductoscopy in comparison with standard diagnostic tests. Onkologie. 2013;36(1-2):12-16. doi:10.1159/000346639 [doi]

8. Bahl M, Baker JA, Greenup RA, Ghate S V. Diagnostic Value of Ultrasound in Female Patients With Nipple Discharge. AJRAmerican J Roentgenol. 2015;205(1):203-208. doi:10.2214/AJR.14.13354 [doi]

9. Sanders LM, Daigle M. The Rightful Role of MRI after Negative Conventional Imaging in the Management of Bloody Nipple Discharge. Breast J. 2016;22(2):209-212. doi:10.1111/tbj.12551 [doi]

10. Galvin R, Joyce D, Downey E, Boland F, Fahey T, Hill AK. Development and validation of a clinical prediction rule to identify suspected breast cancer: a prospective cohort study. BMC Cancer. 2014;14:743. doi:10.1186/1471-2407-14-743 [doi]

11. Lesetedi C, Rayne S, Kruger D, Benn CA. Indicators of breast cancer in patients undergoing microdochectomy for a pathological nipple discharge in a middle-income country. J Surg Res. 2017;220:336-340. doi:S0022-4804(17)30428-6 [pii]

12. van Gelder L, Bisschops RH, Menke-Pluymers MB, Westenend PJ, Plaisier PW. Magnetic resonance imaging in patients with unilateral bloody nipple discharge; useful when conventional diagnostics are negative? World J Surg. 2015;39(1):184-186. doi:10.1007/s00268-014-2701-1 [doi]

13. on Breast Imaging: EP, Lee SJ, Trikha S, et al. ACR Appropriateness Criteria((R)) Evaluation of Nipple Discharge. J Am Coll Radiol. 2017;14(5S):S138-S153. doi:S1546-1440(17)30137-0 [pii]

14. Paula IB de, Campos AM. Breast imaging in patients with nipple discharge. Radiol Bras. 2017. doi:10.1590/0100-3984.2016.0103

15. Yamamoto D, Shoji T, Kawanishi H, et al. A utility of ductography and fiberoptic ductoscopy for patients with nipple discharge. Breast Cancer Res Treat. 2001;70(2):103-108.

16. Yamamoto D, Ueda S, Senzaki H, et al. New diagnostic approach to intracystic lesions of the breast by fiberoptic ductoscopy. Anticancer Res. 2001;21(6A):4113-4116.

17. Okazaki A, Okazaki M, Asaishi K, et al. Fiberoptic ductoscopy of the breast: a new diagnostic procedure for nipple discharge. Jpn J Clin Oncol. 1991;21(3):188-193.

18. Matsunaga T, Ohta D, Misaka T, et al. Mammary ductoscopy for diagnosis and treatment of intraductal lesions of the breast. Breast Cancer. 2001;8(3):213-221.

19. Grunwald S, Heyer H, Paepke S, et al. Diagnostic value of ductoscopy in the diagnosis of nipple discharge and intraductal proliferations in comparison to standard methods. Onkologie. 2007;30(5):243-248 doi:100848 [pii] 
20. Han Y, Li J, Han S, Jia S, Zhang Y, Zhang W. Diagnostic value of endoscopic appearance during ductoscopy in patients with pathological nipple discharge. BMC Cancer. 2017;17(1):300-303. doi:10.1186/s12885017-3288-3 [doi]

21. Kamali S, Bender O, Kamali GH, Aydin MT, Karatepe O, Yuney E. Diagnostic and therapeutic value of ductoscopy in nipple discharge and intraductal proliferations compared with standard methods. Breast Cancer. 2014;21(2):154-161. doi:10.1007/s12282-012-0377-7 [doi]

22. Waaijer L, van Diest PJ, Verkooijen HM, et al. Interventional ductoscopy in patients with pathological nipple discharge. Br J Surg. 2015;102(13):1639-1648. doi:10.1002/bjs.9950 [doi]

23. Jacobs VR, Kiechle M, Plattner B, Fischer T, Paepke S. Breast ductoscopy with a 0.55-mm miniendoscope for direct visualization of intraductal lesions. J Minim Invasive Gynecol. 2005;12(4):359-364. doi:S1553-4650(05)00274-8 [pii]

24. Waaijer L, Simons JM, Borel Rinkes IH, van Diest PJ, Verkooijen HM, Witkamp AJ. Systematic review and meta-analysis of the diagnostic accuracy of ductoscopy in patients with pathological nipple discharge. Br J Surg. 2016;103(6):632-643. doi:10.1002/bjs.10125 [doi]

25. Makita M, Akiyama F, Gomi N, Iwase T. Mammary ductoscopy and watchful follow-up substitute microdochectomy in patients with bloody nipple discharge. Breast Cancer. 2016;23(2):242-251. doi:10.1007/s12282-014-0561-z [doi]

26. Filipe MD, Waaijer L, van der Pol CC, van Diest P, Witkamp AJ. Interventional ductoscopy as an alternative for major duct excision or microdochectomy in women suffering pathological nipple discharge: a single centre experience. Clin Breast Cancer. January 2020. doi:10.1016/j.clbc.2019.12.008

27. de Boorder T, Waaijer L, van Diest PJ, Witkamp AJ. Ex vivo feasibility study of endoscopic intraductal laser ablation of the breast. Lasers Surg Med. 2018;50(2):137-142. doi:10.1002/1sm.22745

28. Hutton B, Salanti G, Caldwell DM, et al. The PRISMA extension statement for reporting of systematic reviews incorporating network meta-analyses of health care interventions: checklist and explanations. Ann Intern Med. 2015;162(11):777-784. doi:10.7326/M14-2385 [doi]

29. Whiting PF, Rutjes AW, Westwood ME, et al. QUADAS-2: a revised tool for the quality assessment of diagnostic accuracy studies. Ann Intern Med. 2011;155(8):529-536. doi:10.7326/0003-4819-155-8201110180-00009 [doi]

30. Egger M, Smith GD, Schneider M, Minder C. Bias in meta-analysis detected by a simple, graphical test. BMJ. 1997;315(7109):629-634. doi:10.1136/bmj.315.7109.629 [doi]

31. Rao AA, Feneis J, Lalonde C, Ojeda-Fournier H. A Pictorial Review of Changes in the BI-RADS Fifth Edition. Radiographics. 2016;36(3):623-639. doi:10.1148/rg.2016150178 [doi]

32. Koerkamp BG, Hunink MG, Stijnen T, Hammitt JK, Kuntz KM, Weinstein MC. Limitations of acceptability curves for presenting uncertainty in cost-effectiveness analysis. Med Decis Making. 2007;27(2):101-111. doi:27/2/101 [pii]

33. Morrogh M, Morris EA, Liberman L, Borgen PI, King TA. The predictive value of ductography and magnetic resonance imaging in the management of nipple discharge. Ann Surg Oncol. 2007;14(12):33693377. doi:10.1245/s10434-007-9530-5 [doi]

34. Denewer A, El-Etribi K, Nada N, El-Metwally M. The role and limitations of mammary ductoscope in management of pathologic nipple discharge. Breast J. 2008;14(5):442-449. doi:10.1111/j.15244741.2008.00620.x [doi]

35. Bender O, Balci FL, Yuney E, Akbulut H. Scarless endoscopic papillomectomy of the breast. Onkologie. 2009;32(3):94-98. doi:10.1159/000195694 [doi] 
36. Vaughan A, Crowe JP, Brainard J, Dawson A, Kim J, Dietz JR. Mammary ductoscopy and ductal washings for the evaluation of patients with pathologic nipple discharge. Breast J. 2009;15(3):254-260. doi:10.1111/j.1524-4741.2009.00714.x [doi]

37. Bahl M, Gadd MA, Lehman CD. JOURNAL CLUB: Diagnostic Utility of MRI After Negative or Inconclusive Mammography for the Evaluation of Pathologic Nipple Discharge. AJRAmerican J Roentgenol. 2017;209(6):1404-1410. doi:10.2214/AJR.17.18139 [doi]

38. Gui G, Agusti A, Twelves D, et al. INTEND II randomized clinical trial of intraoperative duct endoscopy in pathological nipple discharge. Br J Surg. 2018;105(12):1583-1590. doi:10.1002/bjs.10990 [doi]

39. Zacharioudakis K, Kontoulis T, Vella JX, et al. Can we see what is invisible? The role of MRI in the evaluation and management of patients with pathological nipple discharge. Breast Cancer Res Treat. 2019;178(1):115-120. doi:10.1007/s10549-019-05321-w [doi]

40. Bahl M, Baker JA, Greenup RA, Ghate S V. Evaluation of Pathologic Nipple Discharge: What is the Added Diagnostic Value of MRI? Ann Surg Oncol. 2015;22 Suppl 3:S435-41. doi:10.1245/s10434-0154792-9 [doi]

41. Nicholson BT, Harvey JA, Patrie JT, Mugler JP. 3D-MR Ductography and Contrast-Enhanced MR Mammography in Patients with Suspicious Nipple Discharge; A Feasibility Study. Breast J. 2015;21(4):352-362. doi:10.1111/tbj.12417

42. Lorenzon M, Zuiani C, Linda A, Londero V, Girometti R, Bazzocchi M. Magnetic resonance imaging in patients with nipple discharge: should we recommend it? Eur Radiol. 2011;21(5):899-907. doi:10.1007/ s00330-010-2009-y [doi]

43. Orel SG, Dougherty CS, Reynolds C, Czerniecki BJ, Siegelman ES, Schnall MD. MR imaging in patients with nipple discharge: initial experience. Radiology. 2000;216(1):248-254. doi:10.1148/ radiology.216.1.r00jn28248 [doi]

44. Zielinski J, Jaworski R, Irga-Jaworska N, Pikula M, Hunerbein M, Jaskiewicz J. Use of fiberoductoscopy for the management of patients with pathological nipple discharge: experience of a single center in Poland. Breast Cancer. 2018;25(6):753-758. doi:10.1007/s12282-018-0883-3 [doi]

45. Zielinski J, Jaworski R, Irga-Jaworska N, Haponiuk I, Jaskiewicz J. The significance of ductoscopy of mammary ducts in the diagnostics of breast neoplasms. Wideochirurgia $\mathrm{i}$ inne Tech maloinwazyjne = Videosurgery other miniinvasive Tech. 2015;10(1):79-86. doi:10.5114/wiitm.2014.46823 [doi]

46. Chang YK, Chen CTH, Wang M, et al. Could ductoscopy alleviate the need of microdochectomy in pathological nipple discharge? Breast Cancer. February 2020. doi:10.1007/s12282-020-01051-w

47. Waaijer L, Filipe MD, Simons J, et al. Detection of breast cancer precursor lesions by autofluorescence ductoscopy. Breast Cancer. July 2020. doi:10.1007/s12282-020-01136-6

48. Chesebro AL, Chikarmane SA, Ritner JA, Birdwell RL, Giess CS. Troubleshooting to Overcome Technical Challenges in Image-guided Breast Biopsy. RadioGraphics. 2017;37(3):705-718. doi:10.1148/ rg.2017160117

49. Bick U, Trimboli RM, Athanasiou A, et al. Image-guided breast biopsy and localisation: recommendations for information to women and referring physicians by the European Society of Breast Imaging. Insights Imaging. 2020;11(1):12. doi:10.1186/s13244-019-0803-x 


\section{Supplementary data}

\section{Appendix 1: Search strategy}

\section{Pubmed}

(((“Nipple Discharge”[Mesh]) OR nipple discharge*[Title/Abstract])) AND (((OR “Mammography”[Mesh] OR “Magnetic Resonance Imaging”[Mesh] OR "Endoscopy”[Mesh] OR “ductoscopy”[tiab] OR “ductoscope”[tiab])) OR ((Magnetic Resonance Imaging[Title/Abstract] OR MRI[Title/Abstract] OR endoscop*[Title/ Abstract] OR ductoscop*[Title/Abstract] OR fiberoductoscop*[Title/Abstract] OR $\operatorname{FDS}[$ tiab])))

\section{Embase}

('breast discharge'/exp OR 'breast discharge*':ti,ab,kw) AND ('nuclear magnetic resonance imaging'/exp OR 'nuclear magnetic resonance imaging':ti,ab,kw OR 'ductoscop*':ti,ab,kw OR 'endoscopy'/exp OR 'endoscopy':ti,ab,kw OR 'fiberoductoscop*':ti,ab,kw) 


\section{Appendix 2: Supplementary Figures}
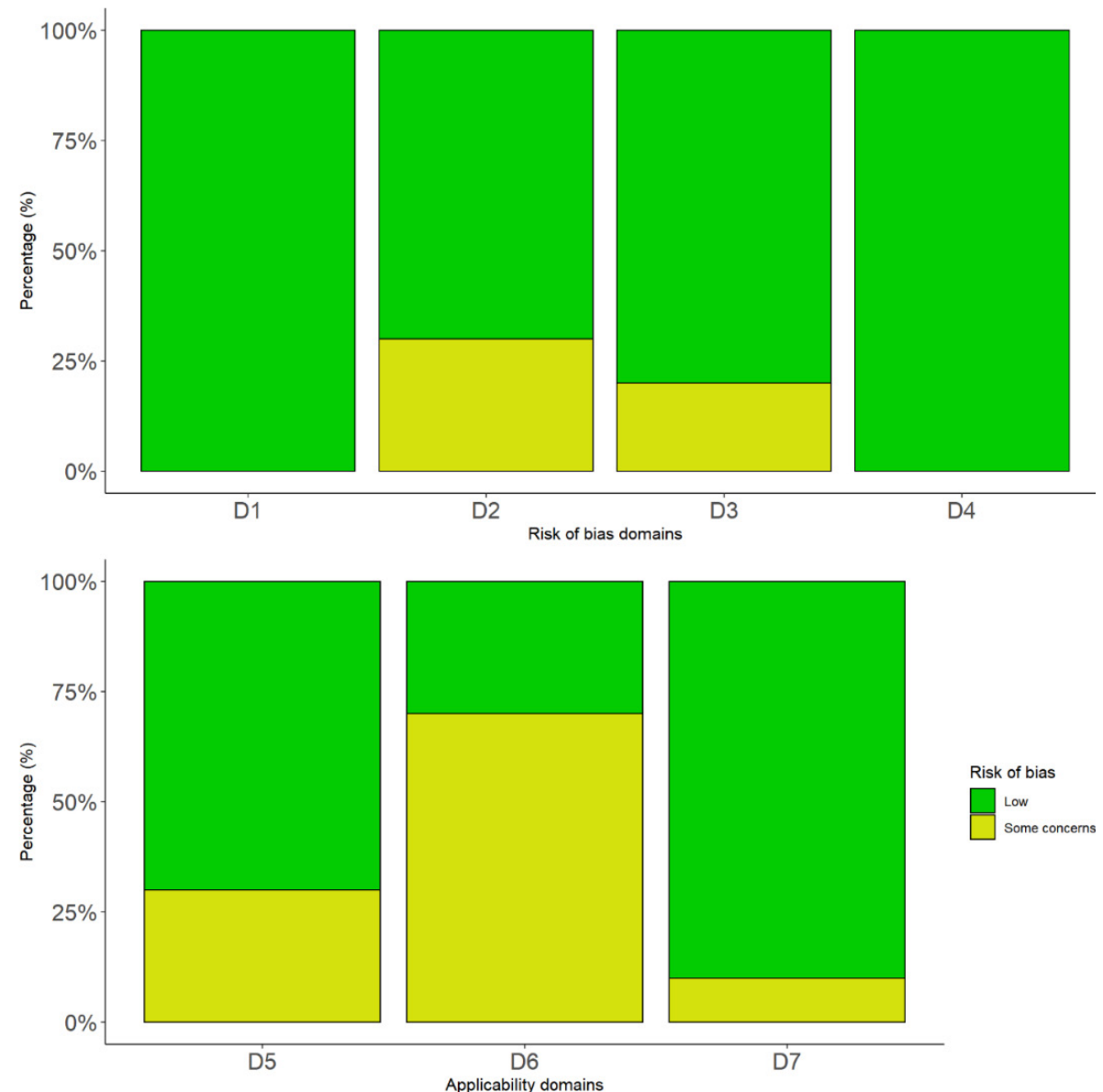

Supplementary figure 1. Summary of the risks of bias and applicability domains. D1= Patient selection; D2 = Index test; D3 = Reference standard; D4 = Flow and timing; D5 = Patient selection; D6 = Index test; D7 = Reference standard. 

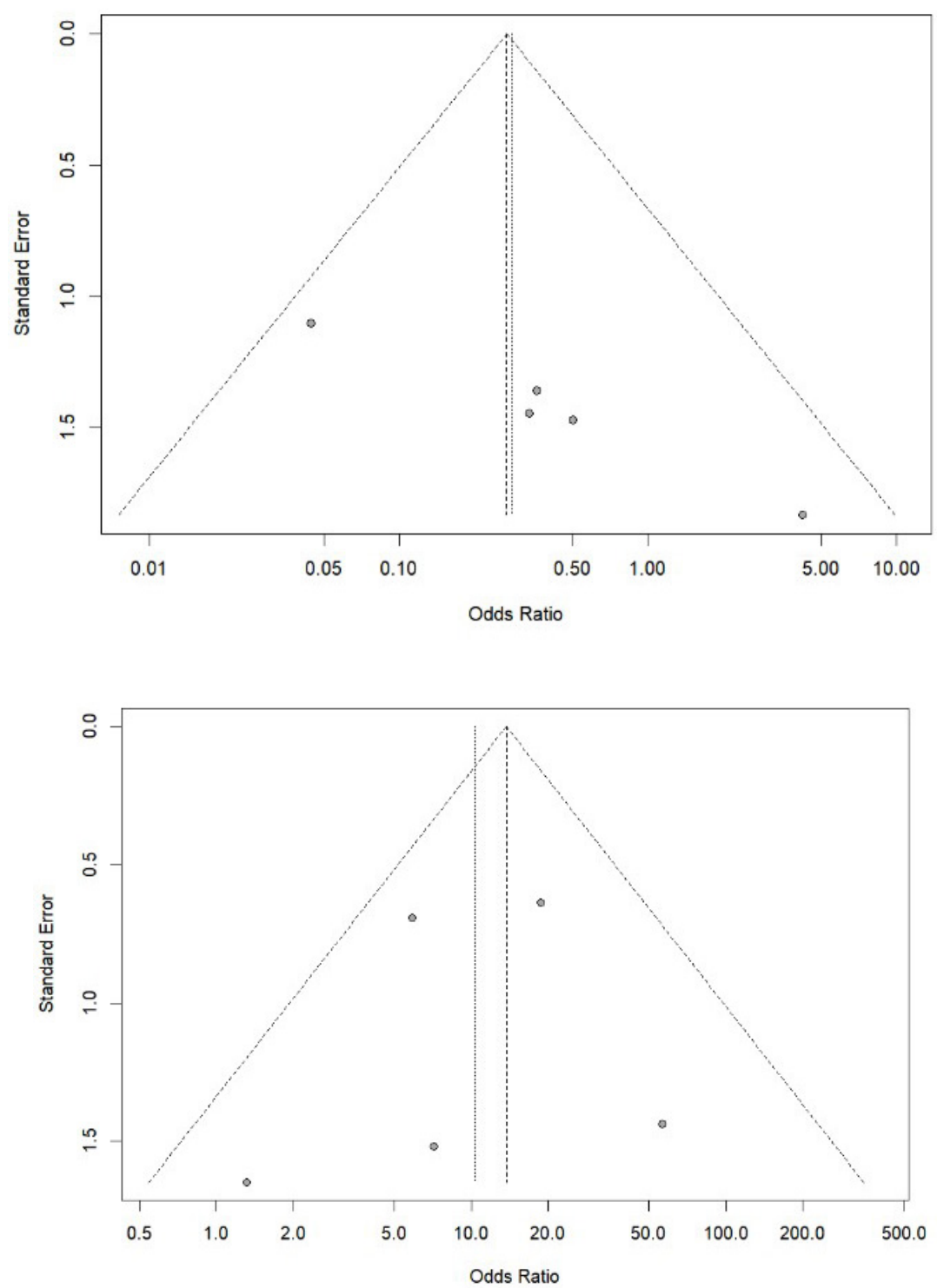

Supplementary figure 2. Funnel plots for sensitivity (top) and specificity (bottom) comparing MRI to ductoscopy in patients with PND without radiological suspicion for malignancy. MRI = Magnetic resonance imaging, PND = Pathological nipple discharge. 


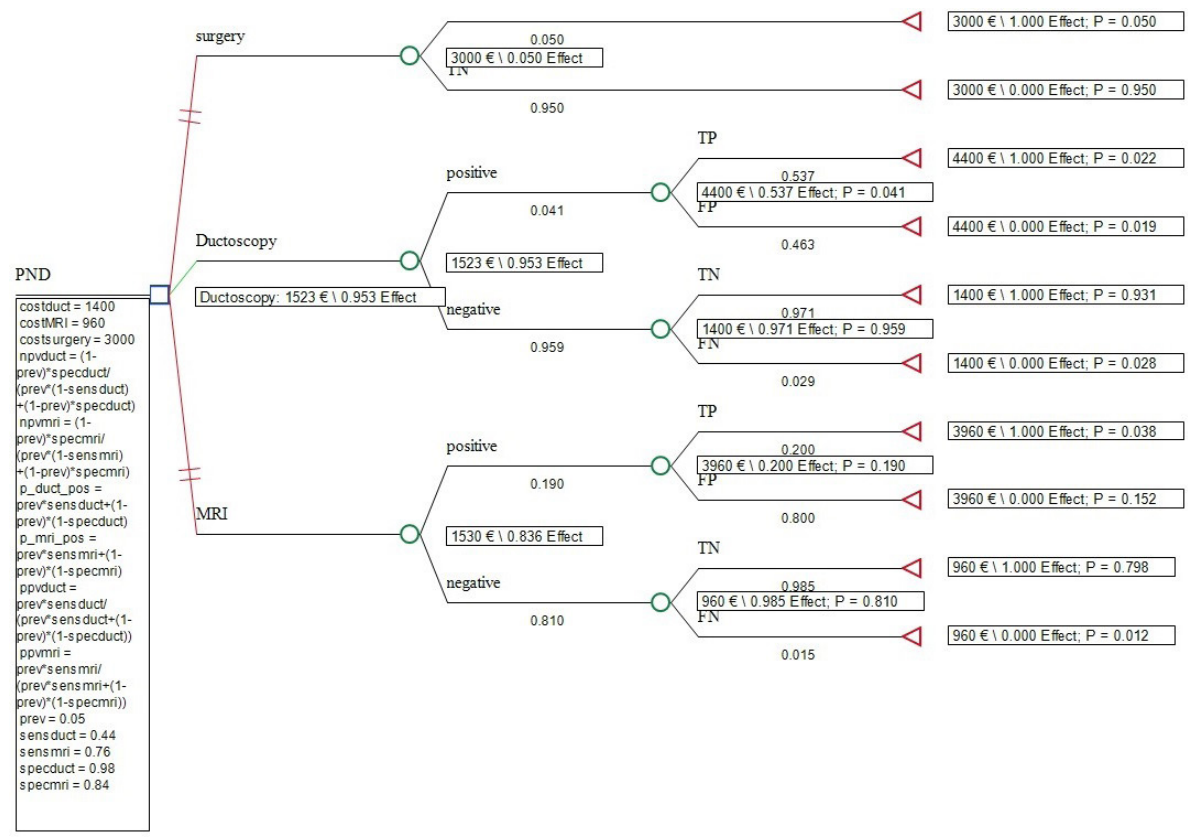

Supplementary Figure 3. Cost-effective analysis of diagnostic performance of ductoscopy, duct excision surgery and MRI for the detection of cancer in patients with PND with negative conventional radiological findings. $\mathrm{PND}=$ Pathological nipple discharge, $\mathrm{MRI}=$ magnetic resonance imaging, $\mathrm{TP}=$ true positive, $\mathrm{FP}=$ False positive, $\mathrm{FN}=$ False negative, $\mathrm{TN}=$ True negative.

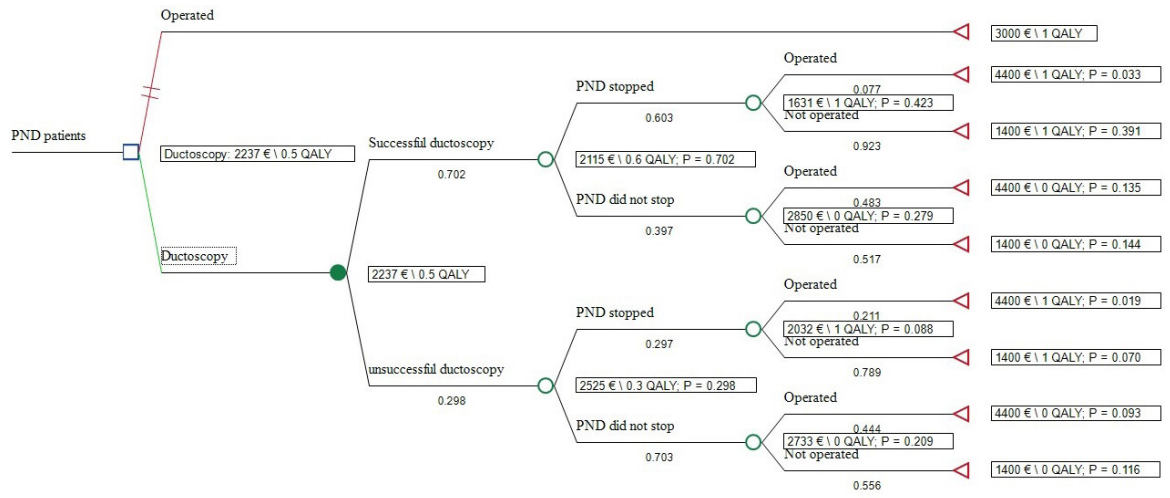

Supplementary Figure 4. Cost-effective analysis of therapeutic performance of ductoscopy and duct excision surgery in patients with PND with negative conventional radiological findings. PND = Pathological nipple discharge 


\section{Supplementary tables}

Supplementary Table 1. Different ICER depending on the sensitivities (based on the $95 \%$ CI) of ductoscopy and MRI for the detection of breast cancer in patients with PND without radiological signs of malignancy.

\begin{tabular}{lllll}
\hline $\begin{array}{l}\text { Sensitivity of } \\
\text { Ductoscopy/MRI }\end{array}$ & $\mathbf{7 1 \%}$ & $\mathbf{7 6 \%}$ & $\mathbf{8 1 \%}$ & $\mathbf{8 6 \%}$ \\
\hline $21 \%$ & -4781.8 & -5061.65 & -5301.66 & -5636.13 \\
$33 \%$ & -4305.5 & -4551.07 & -4773.68 & -5082.32 \\
$44 \%$ & -3815.41 & $-\mathbf{4 0 2 6 . 4 5}$ & -4230.66 & -4512.16 \\
$55 \%$ & -3375.32 & -3557.04 & -3745.16 & -4003.06 \\
$66 \%$ & -2961.47 & -3116.97 & -3290.14 & -3526.26 \\
\hline
\end{tabular}

MRI = Magnetic resonance imaging, ICER = Incremental cost-effectiveness ratio, PND pathological nipple discharge, $\mathrm{CI}=$ confidence interval. Bold is the ICER when pooled sensitivity of ductoscopy and MRI are used.

Supplementary Table 2. Cost-effectiveness analysis comparing ductoscopy to duct excision surgery in patients with PND without radiological signs of malignancy based on the different reported cannulation rates in the known literature.

\begin{tabular}{llll}
\hline Ductoscopy success rate & Effect difference & Cost difference & ICER \\
\hline $70.2 \%$ & -0.39703 & -3441.13 & 8680.677 \\
$75 \%$ & -0.40015 & -3454.84 & 8649.111 \\
$80 \%$ & -0.39941 & -3462.60 & 8680.545 \\
$90 \%$ & -0.39920 & -3509.62 & 8800.454 \\
$95 \%$ & -0.39590 & -3544.97 & 8968.017 \\
$100 \%$ & -0.40092 & -3536.62 & 8831.185 \\
\hline
\end{tabular}

ICER = Incremental cost-effectiveness ratio, PND pathological nipple discharge. 


$$
7
$$




\section{Chapter 7}

\section{Patient-reported outcomes of ductoscopy procedures for pathologic nipple discharge}

M.D. Filipe

J.M. Simons

L. Moeliker

L. Waaijer

M.R. Vriens

P.J. van Diest

A.J. Witkamp 


\section{Abstract}

\section{Background}

Pathologic nipple discharge (PND) is a common complaint often associated with breast cancer. However, when ultrasound and mammography are negative, the chances of malignancy are lower than 5\%. Currently, major duct excision and microdochectomy are often recommended to alleviate symptoms and definitely rule out malignancy, but can cause infections and breastfeeding problems. Ductoscopy is a minimally invasive endoscopy technique that allows visualization of the mammary ducts and may not only obviate surgery but also detect malignancy. The aim of this study was to determine quality of life (QOL) after ductoscopy in patients with PND.

\section{Materials and methods}

All PND patients referred for ductoscopy between 2014 and 2015 to our hospital were included. Ductoscopy procedures were performed under local anaesthesia in the outpatient clinic. Patients were asked to fill out questionnaires (Breast-Q, EQ-5D-5L and SF-36) on the day of ductoscopy, and after 2 weeks, 3 and 6 months. Additionally, we performed reliability analysis to determine if these questionnaires were suitable for PND patients.

\section{Results}

Fifty consecutive patients underwent ductoscopy of whom 47 patients participated in this study. One domain of SF-36 (vitality) varied significantly over time. Breast-Q, SF-36 and EQ-5D-5L showed that QOL after ductoscopy for PND was unaffected by ductoscopy. Success of the ductoscopy procedure was a significant predictor for satisfaction with the result domain.

\section{Conclusion}

Ductoscopy is a minimally invasive technique that does not seem to impact QoL of PND patients over time. Breast-Q, SF-36 and EQ-5D-5L seem to be suitable existing QOL tests for PND patients undergoing ductoscopy, whereas SF-36 would require modifications. 


\section{Introduction}

Pathologic nipple discharge (PND) is the third most common breast-related complaint, after pain and palpable lumps. ${ }^{1}$ PND is defined as unilateral, spontaneous and bloody or serous discharge, usually arising from a single duct orifice of the nipple. PND is regarded as a possible sign of breast cancer and it accounts for 3-5\% of surgical breast clinic referrals. ${ }^{2-5}$ Ultrasound, mammography and magnetic resonance imaging (MRI) often fail to reveal the underlying cause of PND. ${ }^{6,7}$ Therefore, most women suffering from PND undergo invasive surgical procedures, such as microdochectomy or major duct excision, to rule out malignancy and relieve complaints.(6-8) These surgical procedures are performed under general anaesthesia and are associated with scarring, which may result in breastfeeding difficulties in fertile women. ${ }^{8}$ Furthermore, malignancy is found in only $5-8 \%$ of these patient. ${ }^{5,9,10}$ This means around $90-95 \%$ of PND patients undergo surgery for benign causes.

Ductoscopy is a minimally invasive micro-endoscopic technique that may obviate the need for invasive surgery in patients with PND. It enables real-time visualization of the milk ducts of the breast. The procedure can be performed under local anaesthesia at the outpatient clinic and is currently used as a diagnostic tool in the work-up of women suffering from PND without suspicious radiological findings. ${ }^{11-18}$ Previous studies showed the success of ductoscopy in finding the intraductal lesion causing PND before or during duct excision. ${ }^{19-23}$ Moreover, it is possible to remove or laser-destruct intraductal lesions causing PND, thereby preventing surgery, ${ }^{18,24-26}$ and new approaches such as autofluorescence ductoscopy may improve the detection of precancerous lesions in patients with high risk of developing cancer. ${ }^{27-29}$ Despite the increasing relevance of ductoscopy for diagnostic and therapeutic purposes, there are no data available on the impact on the quality of life after ductoscopy. Therefore, we evaluated short- and mid-term impact of ductoscopy on quality of life (QOL) of PND patients. 


\section{Materials and methods}

\section{Patient selection}

This prospective consecutive study included women who presented with unilateral PND between 2014 and 2015 in the University Medical Centre Utrecht (UMCU) in The Netherlands. Part of these patients were reported before. ${ }^{26}$ Inclusion criterion was patients with PND lasting more than at least 3 months. Exclusion criteria for analysis were radiological and/or pathological suspicion of malignancy.

Before ductoscopy, patients underwent imaging consisting of ultrasound and/or mammography. Patients received an additional MRI and/or core needle biopsy (CNB) prior to ductoscopy when there was a palpable mass and/or when ultrasound and/ or mammography were BIRADS IV. When these additional tests were negative for malignancy (thereby downgrading the initial BIRADS classification), these patients were eligible for ductoscopy. Either the UMCU (as tertiary referral hospital) or the referring hospital performed the diagnostic work-up. The Medical Research Ethics Committee (METC) of the UMC Utrecht approved the study.

Patients were asked to fill out the Breast-Q, SF-36 and the EQ-5D-5L QOL questionnaires at baseline (T0, day of the ductoscopy), and at 2 weeks (T1), 3 (T2) and 6 months (T3) after ductoscopy. Written informed consent was obtained for all time points.

\section{Quality of life assessment questionnaires}

\section{Breast- $Q$}

BREAST-Q is a validated and widely used patient-reported outcome QOL instrument that measures patient satisfaction of the breast after surgical procedures. Categories analysed were sexual and psychological well-being, and physical well-being of the chest. Each scale is summarized in a score ranging from 0 (lowest satisfaction) till 100 (highest satisfaction).$^{30}$

\section{$S F-36$}

The Short Form (SF-36) Health Survey is a 36-item, patient-reported survey of general patient health with the following subscales: physical functioning, physical role, bodily pain, social functioning, mental health, emotional status, vitality, and general health perceptions. Each subscale includes 2-10 items and has a score ranging from 0 to 100 . Additionally, there are also two large overall subscales for mental health and physical health. Low scores indicate impaired QOL or impaired quality of a certain subscale. SF-36 has been translated into many languages (including Dutch) and validated per language. ${ }^{31,32}$ 


\section{$E Q-5 D-5 L$}

EQ-5D-5L is a standardized instrument for measuring general health status that consists of descriptive part and a single summary index score. The descriptive part contains five items: mobility, self-care, pain, usual activities and psychological aspects of health. Each of these five items can be scored at five levels $(1=$ no problems, $2=$ slight problems, 3 =moderate problems, $4=$ severe problems, 5 =extreme problems). These results are weighted into yield a single summary index score ranging from 0 (worst health) to 1 (best health). The second part of the EQ-5D-5L is the EQ visual analogue scale (VAS) consisting of a $20-\mathrm{cm}$ vertical scale, with a range of 0 (worst health perceived) to 100 points (best health perceived). ${ }^{33}$

\section{Validation of questionnaires of patients undergoing ductoscopy}

Since none of the above questionnaires had been validated for PND patients, we performed reliability analysis by measuring the standardised alpha of each of the domains of the questionnaires. Next, we determined which of the items of the various domains were specifically useful for PND patients. Furthermore, a split-half reliability analysis was performed to further determine internal reliability of the questionnaires. The fact that we asked patients to fill out questionnaires at four different time points allowed a test-retest analysis to determine external reliability of the questionnaires in PND patients.

\section{Statistics}

Normality was determined using Kurtosis, in which z values between -3 and 3 were considered as normally distributed data. Normally distributed continuous data were described by means and standard deviations (SD). In non-continuous not normally distributed data, median and interquartile range were used to describe the data. P values below 0.05 were considered to be significant.

In the longitudinal analysis, General Linear Mixed Models was used to determine and compare quality of life over time correcting for possible confounding factors such as age, PND symptoms, final diagnosis made by ductoscopy and/or surgery (benign or malignant) and whether the ductoscopy succeeded or not. Split half correlation analysis and calculation of Cochran's alpha were performed in order to assess internal reliability. Test-retest using Pearson's correlation analysis was performed to determine external validity by comparing the test results on the different follow-up periods. Statistical analysis of the database was performed using RStudio 1.2.5001 (with R version: $\times 64$ 3.6.2). Statistical packages used were nmle, tydir, HRQoL, lbscorer and psych. 


\section{Results}

Between 2014 and 2015, 50 patients with PND underwent ductoscopy and agreed to fill out questionnaires. Three of these ended up not filling out any of the questionnaires leaving 47 patients for analysis. Table 1 shows the baseline characteristics. The mean age was 51.7 (ranging from 27 to 83 ). Ductoscopy was successful in $72.3 \%$ of cases.

\section{Breast-Q}

Table 2 shows the average scores per Breast-Q domain over the follow-up period. Average psychosocial well-being was 69.7 out of 100 at baseline and $66.4,70.3$ and 69.8 at 2 weeks, 3 and 6 months after ductoscopy, respectively. Mean physical well-being of the chest was 73.8 out of 100 at baseline, 73.0 after 2 weeks, 78.7 after three and 78.9 after 6 months. Patients scored 29.6 out of 100 for sexual well-being on the day of ductoscopy, 30.4 after 2 weeks, 20.1 after three and 22.8 after 6 months. Satisfaction with the result was scored on average 53.0 points 2 weeks after ductoscopy, 50.0 after 3 and 47.0 after 6 months. None of the domains showed statistically significant differences over time after correction for age, whether or not PND prevailed, diagnosis after possible surgery and success of ductoscopy. However, success of ductoscopy was a significant predictor of satisfaction with the result domain.

\section{EQ-5D-5L}

Table 2 displays mean scores for the EQ-5D-5L questionnaires. Average descriptive score was 86.2 out of 100 in patients at baseline, 86.5, 2 weeks after ductoscopy, 84.9 after three and 90.8 after 6 months. Mean VAS score was 81.4 on the day of the ductoscopy, 79.2, 2 weeks later, 76.6 three and 80.7, six months after ductoscopy. There were no statistically significant differences in scores during follow-up. Age, PND, possible surgery, diagnosis of possible surgery and success of ductoscopy were not confounders for the EQ-5D-5L. 
Table 1. Baseline characteristics of 47 patients with pathologic nipple discharge studied for quality of life after ductoscopy

\begin{tabular}{|c|c|c|}
\hline & & $\mathrm{N}=47$ patients \\
\hline Age years (SD) & & $51.7(11.9)$ \\
\hline \multirow[t]{3}{*}{ Affected breast, $\mathrm{N}(\%)$} & Left & $19(40.4 \%)$ \\
\hline & Right & $24(51.1 \%$ \\
\hline & Both & $4(8.5 \%)$ \\
\hline \multirow[t]{2}{*}{ Successful ductoscopy, N (\%) } & Yes & $34(72.3 \%)$ \\
\hline & No & $13(27.7 \%)$ \\
\hline \multirow[t]{6}{*}{ Diagnosis of ductoscopy, N (\%) } & Normal & $10(21.3 \%)$ \\
\hline & Papilloma & $11(23.4 \%)$ \\
\hline & Epithelial lesions & $3(6.4 \%)$ \\
\hline & Suspicious for malignancy & $5(10.6 \%)$ \\
\hline & other & $5(10.6 \%)$ \\
\hline & NA & $13(27.7 \%)$ \\
\hline \multirow[t]{4}{*}{ Ultrasound BI-RADS classification, N (\%) } & BI-RADS I & $18(38.3 \%)$ \\
\hline & BI-RADS II & $20(42.6 \%)$ \\
\hline & BI-RADS III & $1(2.1 \%)$ \\
\hline & Not performed & $8(17.0 \%)$ \\
\hline \multirow[t]{5}{*}{ Mammography BI-RADS classification, N (\%) } & BI-RADS I & $33(70.2 \%)$ \\
\hline & BI-RADS II & $9(19.1 \%)$ \\
\hline & BI-RADS III & $2(4.3 \%)$ \\
\hline & BI-RADS IV & $1(2.1 \%)$ \\
\hline & Not performed & $2(4.3 \%)$ \\
\hline \multirow[t]{5}{*}{ MRI BI-RADS classification, N (\%) } & BI-RADS I & $7(14.7 \%)$ \\
\hline & BI-RADS II & $5(10.6 \%)$ \\
\hline & BI-RADS III & $2(4.3 \%)$ \\
\hline & BI-RADS IV & $1(2.1 \%)$ \\
\hline & Not performed & $32(68.1 \%)$ \\
\hline \multirow[t]{3}{*}{ Diagnosis after possible surgery, $\mathrm{N}(\%)$} & Benign & $6(12.8 \%)$ \\
\hline & Malignant & $4(8.5 \%)$ \\
\hline & Not performed & $37(78.7 \%)$ \\
\hline \multirow[t]{2}{*}{ PND stopped after (attempted) ductoscopy, N (\%) } & Yes & $18(38.3 \%)$ \\
\hline & No & $29(61.7 \%)$ \\
\hline
\end{tabular}

$\mathrm{SD}=$ standard deviation, BI-RADS = Breast Imaging Reporting and Data System, MRI = magnetic resonance imaging, NA = not applicable 
Table 2. Results of 3 questionnaires (Breast-Q, EQ-5D-5L, SF-36) to assess quality of life over time of patients with pathologic nipple discharged undergoing ductoscopy.

\begin{tabular}{|c|c|c|c|c|c|c|c|}
\hline & $\begin{array}{c}\text { T0 } \\
\text { Mean (SD) } \\
\end{array}$ & $\begin{array}{c}\text { T1 } \\
\text { Mean (SD) } \\
\end{array}$ & P-value & $\begin{array}{c}\text { T2 } \\
\text { Mean (SD) }\end{array}$ & p-value & $\begin{array}{c}\text { T3 } \\
\text { Mean (SD) } \\
\end{array}$ & p-value \\
\hline \multicolumn{8}{|l|}{ Breast-Q } \\
\hline A & $69.7(16.17)$ & $66.4(16.3)$ & 0.132 & $70.35(20.67)$ & 0.689 & $69.76(17.04)$ & 0.971 \\
\hline B & $73.79(15.08)$ & $73(18.01)$ & 0.145 & 78.7 (18.73) & 0.378 & $78.88(12.94)$ & 0.478 \\
\hline $\mathrm{C}$ & $29.57(13.63)$ & $30.36(16.69)$ & 0.149 & $20.14(15.11)$ & 0.157 & $22.75(15.46)$ & 0.300 \\
\hline $\mathrm{D}$ & NA & $53.03(24.3)$ & NA & $50.04(20.23)$ & 0.432 & $47.00(27.98)$ & 0.497 \\
\hline \multicolumn{8}{|l|}{ EQ-5D-5L } \\
\hline EQ-5D $(* 100)$ & $86.24(14.49)$ & 86.53 (13.9) & 0.875 & $84.89(17.72)$ & 0.447 & $90.79(10.29)$ & 0.381 \\
\hline VAS & $81.43(11.54)$ & $79.2(12.13)$ & 0.256 & $76.57(13.62)$ & 0.075 & $80.71(13.08)$ & 0.378 \\
\hline \multicolumn{8}{|l|}{ SF-36 } \\
\hline $\mathrm{PF}$ & $92.50(8.11)$ & $92.8(7.92)$ & 0.700 & $87.95(18.5)$ & 0.305 & 91.18 (11.93) & 0.444 \\
\hline $\mathrm{RP}$ & $71.43(38.32)$ & $72(40.39)$ & 0.919 & $75(43.3)$ & 0.604 & $75(41.46)$ & 0.184 \\
\hline $\mathrm{BP}$ & $73.32(19.07)$ & $74.36(22.1)$ & 0.891 & $80.74(20.36)$ & 0.089 & $81.53(17.5)$ & 0.218 \\
\hline $\mathrm{GH}$ & $72.94(18.69)$ & $71.2(19.05)$ & 0.818 & $68(21.31)$ & 0.296 & $70.18(21.1)$ & 0.320 \\
\hline$V T$ & $66.25(17.83)$ & $58(17.74)$ & 0.001 & $60.87(19.81)$ & 0.014 & $62.19(17.41)$ & 0.048 \\
\hline SF & $86.61(16.29)$ & $84(20.58)$ & 0.063 & $86.41(23.21)$ & 0.323 & $89.71(14.14)$ & 0.523 \\
\hline $\mathrm{RE}$ & $80.95(36.77)$ & $77.33(36.92)$ & 0.299 & $74.6(43.34)$ & 0.506 & $80.39(35.47)$ & 0.655 \\
\hline $\mathrm{MH}$ & $75.14(13.96)$ & $73(20.23)$ & 0.399 & $71.48(20.76)$ & 0.273 & $75.75(15.63)$ & 0.970 \\
\hline
\end{tabular}

Breast-Q = Breast questionnaire, EQ-5D-5L = European Quality of Life, SF-36 = Short form health survey, VAS = Visual analogue score, $\mathrm{PF}=$ Physical functioning, $\mathrm{RP}=$ Role physical, $\mathrm{BP}=$ Bodily pain, $\mathrm{GH}=\mathrm{General}$ health, $\mathrm{VT}=$ Vitality, $\mathrm{SF}=$ Social functioning, $\mathrm{RE}=$ Role emotional, $\mathrm{MH}=$ Mental health, $\mathrm{PCS}=$ [overall] Physical health, MCS = [overall] Mental health, A = PsychoSocial Well-being B = Physical Well-being: Chest, C = Sexual Well-being, $\mathrm{D}=$ Satisfaction with outcome, $\mathrm{T} 0$ = Day of ductoscopy, $\mathrm{T} 1$ = Two weeks after ductoscopy, $\mathrm{T} 2$ = three months after ductoscopy, T3 = Six months after ductoscopy, SD = standard deviation, NA = not applicable, cursive $=$ significant differences and $\mathrm{p}$-value $=$ differences compared to survey at $\mathrm{T} 0$ and are corrected for age, PND symptoms after ductoscopy, whether or not operated and diagnosis in operated patients. 


\section{SF-36}

Table 2 shows the average sum scores of the different domains of the SF-36 questionnaires. Physical functioning, role physical scores, bodily pain, general health, social functioning, role emotional scores and mental health showed no statistical differences in scores during follow-up. Vitality scores dropped statistically significantly from 66.2 at baseline to 58.0 after 2 weeks, 60.9, three months and 62.2 after 6 months. Age was only a significant confounder for the physical functioning domain. Success of ductoscopy, diagnosis after possible operation, whether or not PND stopped and whether or not a patient was operated did not statistically significant influence on any of the SF-36 domains.

\section{Internal and external reliability analysis}

Table 3 shows the results of the internal and external reliability analysis. For BREAST-Q, reliability for psychosocial and physical well-being did not increase if individual items were dropped. Test-retest correlations of psychosocial, physical and sexual well-being and satisfaction of outcome were $0.68,0.79,0.67$ and 0.63 , respectively. Two items ("Comfortable/at ease during sexual activity" and "Confident sexually") correlated negatively with sexual well-being domain and if the former would be dropped, standardised alpha would increase. If the item "I would encourage other women in my situation to have breast reconstruction surgery" would be dropped, the reliability of satisfaction of outcome would increase. 
Table 3. Internal and external validity of three quality of life questionnaires in patients with PND undergoing ductoscopy

\begin{tabular}{llll}
\hline & Std. alpha & Split half reliability & Test-retest reliability \\
\hline Breast-Q & & & \\
A & 0.92 & 0.80 & 0.68 \\
B & 0.93 & 0.68 & 0.79 \\
C & 0.65 & 0.52 & 0.67 \\
D & 0.86 & 0.78 & 0.63 \\
EQ-5D-5L & & & \\
EQ-5D & 0.51 & 0.46 & 0.75 \\
VAS & NA & NA & 0.78 \\
SF-36 & & & \\
PF & 0.86 & 0.58 & 0.70 \\
RP & 0.87 & 0.74 & 0.92 \\
BP & 0.76 & 0.61 & 0.72 \\
GH & 0.82 & 0.21 & 0.76 \\
VT & 0.66 & -0.41 & 0.79 \\
SF & 0.82 & -0.69 & 0.85 \\
RE & 0.90 & 0.77 & 0.55 \\
MH & 0.85 & -0.41 & 0.87 \\
\hline
\end{tabular}

Breast-Q = Breast questionnaire, EQ-5D-5L = European Quality of Life, SF-36 = Short form health survey, VAS $=$ Visual analogue score, $\mathrm{PF}=$ Physical functioning, $\mathrm{RP}=$ Role physical, $\mathrm{BP}=$ Bodily pain, $\mathrm{GH}=\mathrm{General}$ health, $\mathrm{VT}=$ Vitality, $\mathrm{SF}=$ Social functioning, $\mathrm{RE}=$ Role emotional, $\mathrm{MH}=$ Mental health, PCS $=$ [overall] Physical health, MCS = [overall] Mental health, A = PsychoSocial Well-being B = Physical Well-being: Chest, $\mathrm{C}=$ Sexual Well-being, $\mathrm{D}=$ Satisfaction with outcome, NA = Not applicable. 
For EQ-5D-5L, the item "mobility" was not taken into the analysis because there was no variance. If the "anxiety and depression" item would be dropped, the standardised alpha would increase. Split test-retest analysis determining external validity showed a correlation of 0.75 for the descriptive part and 0.78 on the VAS part.

For SF-36, dropping individual physical functioning items would not increase reliability. If the first item of role physical ("Cut down the amount of time you spent on work or other activities") would be dropped, the standardised alpha would increase. If the first item of role emotional ("Cut down the amount of time you spent on work or other activities") would be dropped, the standardised alpha would increase. Dropping individual items of general health would not increase internal validity. However, the second ("I seem to get sick a little easier than other people") and fourth ("I expect my health to get worse") items showed strong negative correlations. Dropping individual items would not improve reliability of the social functioning domain. The first item of social functioning ("During the past 4 weeks, to what extent has your physical health or emotional problems interfered with your normal social activities with family, friends, neighbours, or groups") correlated strongly negative with the domain. For bodily pain, dropping an individual item would not improve reliability. For vitality, the two first items ("Did you feel full of pep?" and "Did you have a lot of energy?") were strongly negative correlated and if the former was dropped, the domain would be more reliable. For mental health, dropping the first item ("Have you been a very nervous person?") would increase reliability while other items ("Have you felt calm and peaceful?" and "Have you been a happy person?") negatively correlated to the mental health score. Test-retest analysis showed a correlation of 0.87 for external validity. 


\section{Discussion}

In this study, we evaluated QOL of patients with PND that underwent ductoscopy. No statistically significant changes were observed in the overall scores of the Breast-Q, SF36 and EQ-5D-5L questionnaires at baseline compared to 2 weeks, 3 and 6 months after ductoscopy, indicating that ductoscopy does not negatively influence QOL. These findings further support the use of ductoscopy, which is performed more and more for both diagnostic and therapeutic purposes. ${ }^{26,34,35}$ Additionally, this study showed that the all domains of Breast-Q, and EQ-5D-5L were stable over time and are thereby useful questionnaires for PND patients, while SF-36 was as a whole less useful and would require modifications.

For Breast-Q the average patient was satisfied with the outcome, with scores higher than reported by studies that assessed QOL of patients undergoing breast conserving surgery, ${ }^{36,37}$ and all domains scored well in internal and external validity. Regarding average sexual well-being, this was reported to be stably low in our study, likely associated with PND itself. For EQ-5D-5L, both descriptive scores and VAS scores were stable over time. For SF-36, the vitality domain showed low reliability scores, and omitting this domain would make this questionnaire more useful for PND patients. For all questionnaires, scores for ductoscopy were overall similar to or higher than breast conserving therapy, ${ }^{38-41}$ favouring ductoscopy as a procedure. To the best of our knowledge, there are no studies describing QOL in PND patients undergoing surgery.

Ductoscopy is often used to detect the cause underlying PND, which in the majority of cases is a benign lesion. ${ }^{23,35,42-45}$ A meta-analysis showed that ductoscopy has a sensitivity of $94 \%$ in detecting intraductal lesions of any type, but cannot reliably discriminate between benign and malignant lesions. ${ }^{46}$ Another network meta-analysis showed that ductoscopy has a significantly higher diagnostic accuracy than MRI for the detection of malignancy in patients with PND and negative ultrasound and/or mammography. ${ }^{47}$ Therefore, ductoscopy is more useful when conventional imaging is negative. ${ }^{26} \mathrm{New}$ techniques in ductoscopy are currently being developed and explored to improve the sensitivity for the detection of (pre)malignant lesions. One of these examples is combining ductoscopy with auto-fluorescence imaging. ${ }^{27-29}$ This addition to ductoscopy may help to detect precancerous lesions in high-risk breast cancer groups since auto-fluorescence has shown to be effective for the detection of precancerous lesions of epithelial origin (such as oesophageal, lung, bronchial and colon cancers). ${ }^{48-52}$ Additionally, the development of therapeutic options such as intraductal laser ablation further increase the utility of ductoscopy. ${ }^{53}$ This means that there is an increasing potential for ductoscopy not only for diagnostic but also for therapeutic purposes. Invasive surgery can be avoided in the majority of patients with PND that undergo ductoscopy as the initial procedure which is a major advantage. ${ }^{26}$ 
Limitations of this study are our relatively small study population and the relatively short follow-up period. Nevertheless, this is the first study to report on QOL following ductoscopy and we do not expect any significant changes in QOL beyond 6 months, since ductoscopy is a minimally invasive procedure.

To conclude, ductoscopy is a minimally invasive endoscopic technique that is currently used to detect, and sometimes treat, intraductal lesions causing PND. This QOL study further supports the use of ductoscopy, as it shows no negative influence on short or mid-term QOL in PND patients. Breast-Q and EQ-5D-5L appear to be useful existing questionnaires for the assessment of QOL in PND patients, while SF-36 would require modifications. Nevertheless, development of a questionnaire specific to PND patients may even be better. 


\section{References}

1. Hussain AN, Policarpio C, Vincent MT. Evaluating nipple discharge. Obstet Gynecol Surv. 2006;61(4):278-283. doi:0006254-200604000-00025 [pii]

2. Dixon JM, Mansel RE. ABC of breast diseases. Symptoms assessment and guidelines for referral. BMJ. 1994;309(6956):722-726.

3. Seltzer MH. Breast complaints, biopsies, and cancer correlated with age in 10,000 consecutive new surgical referrals. Breast J. 2004;10(2):111-117. doi:21284 [pii]

4. Santen RJ, Mansel R. Benign breast disorders. N Engl J Med. 2005;353(3):275-285. doi:353/3/275 [pii]

5. King TA, Carter KM, Bolton JS, Fuhrman GM. A simple approach to nipple discharge. Am Surg. 2000;66(10):960-966.

6. Park CJ, Kim EK, Moon HJ, Yoon JH, Kim MJ. Reliability of Breast Ultrasound BI-RADS Final Assessment in Mammographically Negative Patients with Nipple Discharge and Radiologic Predictors of Malignancy. J Breast Cancer. 2016;19(3):308-315. doi:10.4048/jbc.2016.19.3.308 [doi]

7. van Gelder L, Bisschops RH, Menke-Pluymers MB, Westenend PJ, Plaisier PW. Magnetic resonance imaging in patients with unilateral bloody nipple discharge; useful when conventional diagnostics are negative? World J Surg. 2015;39(1):184-186. doi:10.1007/s00268-014-2701-1 [doi]

8. Sarakbi W Al, Worku D, Escobar PF, Mokbel K. Breast papillomas: current management with a focus on a new diagnostic and therapeutic modality. Int Semin Surg Oncol. 2006;3:1. doi:1477-7800-3-1 [pii]

9. Galvin R, Joyce D, Downey E, Boland F, Fahey T, Hill AK. Development and validation of a clinical prediction rule to identify suspected breast cancer: a prospective cohort study. BMC Cancer. 2014;14:743. doi:10.1186/1471-2407-14-743 [doi]

10. Lesetedi C, Rayne S, Kruger D, Benn CA. Indicators of breast cancer in patients undergoing microdochectomy for a pathological nipple discharge in a middle-income country. J Surg Res. 2017;220:336-340. doi:S0022-4804(17)30428-6 [pii]

11. Yamamoto D, Shoji T, Kawanishi H, et al. A utility of ductography and fiberoptic ductoscopy for patients with nipple discharge. Breast Cancer Res Treat. 2001;70(2):103-108.

12. Yamamoto D, Ueda S, Senzaki H, et al. New diagnostic approach to intracystic lesions of the breast by fiberoptic ductoscopy. Anticancer Res. 2001;21(6A):4113-4116.

13. Okazaki A, Okazaki M, Asaishi K, et al. Fiberoptic ductoscopy of the breast: a new diagnostic procedure for nipple discharge. Jpn J Clin Oncol. 1991;21(3):188-193.

14. Matsunaga T, Ohta D, Misaka T, et al. Mammary ductoscopy for diagnosis and treatment of intraductal lesions of the breast. Breast Cancer. 2001;8(3):213-221.

15. Grunwald S, Heyer H, Paepke S, et al. Diagnostic value of ductoscopy in the diagnosis of nipple discharge and intraductal proliferations in comparison to standard methods. Onkologie. 2007;30(5):243-248 doi: 100848 [pii]

16. Han Y, Li J, Han S, Jia S, Zhang Y, Zhang W. Diagnostic value of endoscopic appearance during ductoscopy in patients with pathological nipple discharge. BMC Cancer. 2017;17(1):300-303. doi:10.1186/s12885017-3288-3 [doi]

17. Kamali S, Bender O, Kamali GH, Aydin MT, Karatepe O, Yuney E. Diagnostic and therapeutic value of ductoscopy in nipple discharge and intraductal proliferations compared with standard methods. Breast Cancer. 2014;21(2):154-161. doi:10.1007/s12282-012-0377-7 [doi] 
18. Waaijer L, van Diest PJ, Verkooijen HM, et al. Interventional ductoscopy in patients with pathological nipple discharge. Br J Surg. 2015;102(13):1639-1648. doi:10.1002/bjs.9950 [doi]

19. Jacobs VR, Kiechle M, Plattner B, Fischer T, Paepke S. Breast ductoscopy with a 0.55-mm miniendoscope for direct visualization of intraductal lesions. J Minim Invasive Gynecol. 2005;12(4):359-364. doi:S1553-4650(05)00274-8 [pii]

20. Ling H, Liu GY, Lu JS, et al. Fiberoptic ductoscopy-guided intraductal biopsy improve the diagnosis of nipple discharge. Breast J. 2009;15(2):168-175. doi:10.1111/j.1524-4741.2009.00692.x [doi]

21. Zhu X, Xing C, Jin T, Cai L, Li J, Chen Q. A randomized controlled study of selective microdochectomy guided by ductoscopic wire marking or methylene blue injection. Am J Surg. 2011;201(2):221-225. doi:10.1016/j.amjsurg.2010.03.011 [doi]

22. Moncrief RM, Nayar R, Diaz LK, Staradub VL, Morrow M, Khan SA. A comparison of ductoscopyguided and conventional surgical excision in women with spontaneous nipple discharge. Ann Surg. 2005;241(4):575-581. doi:00000658-200504000-00006 [pii]

23. Bender O, Balci FL, Yuney E, Akbulut H. Scarless endoscopic papillomectomy of the breast. Onkologie. 2009;32(3):94-98. doi:10.1159/000195694 [doi]

24. Makita M, Akiyama F, Gomi N, Iwase T. Mammary ductoscopy and watchful follow-up substitute microdochectomy in patients with bloody nipple discharge. Breast Cancer. 2016;23(2):242-251. doi:10.1007/s12282-014-0561-z [doi]

25. Chang YK, Chen CTH, Wang M, et al. Could ductoscopy alleviate the need of microdochectomy in pathological nipple discharge? Breast Cancer. Published online February 1, 2020. doi:10.1007/s12282020-01051-w

26. Filipe MD, Waaijer L, van der Pol CC, van Diest P, Witkamp AJ. Interventional ductoscopy as an alternative for major duct excision or microdochectomy in women suffering pathological nipple discharge: a single centre experience. Clin Breast Cancer. Published online January 27, 2020. doi:10.1016/j. clbc.2019.12.008

27. Jacobs VR, Paepke S, Schaaf H, Weber BC, Kiechle-Bahat M. Autofluorescence ductoscopy: a new imaging technique for intraductal breast endoscopy. Clin Breast Cancer. 2007;7(8):619-623. doi:S1526-8209(11)70750-9 [pii]

28. Douplik A, Leong WL, Easson AM, Done S, Netchev G, Wilson BC. Feasibility study of autofluorescence mammary ductoscopy. J Biomed Opt. 2009;14(4):44036. doi:10.1117/1.3210773 [doi]

29. Waaijer L, Filipe MD, Simons J, et al. Detection of breast cancer precursor lesions by autofluorescence ductoscopy. Breast Cancer. Published online July 28, 2020. doi:10.1007/s12282-020-01136-6

30. Pusic AL, Klassen AF, Scott AM, Klok JA, Cordeiro PG, Cano SJ. Development of a new patient-reported outcome measure for breast surgery: The BREAST-Q. Plast Reconstr Surg. 2009;124(2):345-353. doi:10.1097/PRS.0b013e3181aee807

31. Jr JEW. SF-36 health survey update. Spine (Phila Pa 1976). 2000;25(24):3130-3139. doi:10.1097/00007632-200012150-00008 [doi]

32. Brazier J, Roberts J, Deverill M. The estimation of a preference-based measure of health from the SF-36. J Health Econ. 2002;21(2):271-292. doi:S0167-6296(01)00130-8 [pii]

33. Herdman M, Gudex C, Lloyd A, et al. Development and preliminary testing of the new five-level version of EQ-5D (EQ-5D-5L). Qual Life Res. 2011;20(10):1727-1736. doi:10.1007/s11136-011-9903-x [doi]

34. Liu M, Guo G, Xie F, Wang S, Yang H, Wang S. Mammary ductoscopy and follow-up avoid unnecessary duct excision in patients with pathologic nipple discharge. J Surg Oncol. 2015;112(2):139-143. doi:10.1002/jso.23972 [doi] 
35. Gui G, Agusti A, Twelves D, et al. INTEND II randomized clinical trial of intraoperative duct endoscopy in pathological nipple discharge. Br J Surg. 2018;105(12):1583-1590. doi:10.1002/bjs.10990 [doi]

36. Krzos A, Stanislawek A, Jedrych M, Luczyk M, Slusarska B. Satisfaction with the Aesthetic Effect and Quality of Life for Women after Breast Conserving Therapy (BCT)-Preliminary Research. Int J Environ Res Public Health. 2019;16(23):10.3390/ijerph16234682. doi:E4682 [pii]

37. Hallberg H, Elander A, Kolby L, Hansson E. A biological or a synthetic mesh in immediate breast reconstruction? A cohort-study of long-term Health related Quality of Life (HrQoL). Eur J Surg Oncol. 2019;45(10):1812-1816. doi:S0748-7983(19)30338-5 [pii]

38. Lagendijk M, van Egdom LSE, van Veen FEE, et al. Patient-Reported Outcome Measures May Add Value in Breast Cancer Surgery. Ann Surg Oncol. 2018;25(12):3563-3571. doi:10.1245/s10434-018-6729-6 [doi]

39. Lagendijk M, Vos EL, Nieboer D, Verhoef C, Corten EML, Koppert LB. Evaluation of cosmetic outcome following breast-conserving therapy in trials: panel versus digitalized analysis and the role of PROMs. Breast J. 2018;24(4):519-525. doi:10.1111/tbj.12980 [doi]

40. Freitas-Silva R, Conde DM, de Freitas-Junior R, Martinez EZ. Comparison of quality of life, satisfaction with surgery and shoulder-arm morbidity in breast cancer survivors submitted to breast-conserving therapy or mastectomy followed by immediate breast reconstruction. Clinics (Sao Paulo). 2010;65(8):781-787. doi:S1807-59322010000800007 [pii]

41. Lindegren A, Schultz I, Wickman M. Improved patient-reported outcomes after autologous fat transplantation and corrective surgery after breast surgery. J Plast Surg Hand Surg. 2019;53(2):111-118. doi: 10.1080/2000656X.2018.1561456 [doi]

42. Zielinski J, Jaworski R, Irga-Jaworska N, Pikula M, Hunerbein M, Jaskiewicz J. Use of fiberoductoscopy for the management of patients with pathological nipple discharge: experience of a single center in Poland. Breast Cancer. 2018;25(6):753-758. doi:10.1007/s12282-018-0883-3 [doi]

43. Vaughan A, Crowe JP, Brainard J, Dawson A, Kim J, Dietz JR. Mammary ductoscopy and ductal washings for the evaluation of patients with pathologic nipple discharge. Breast J. 2009;15(3):254-260. doi:10.1111/j.1524-4741.2009.00714.x [doi]

44. Denewer A, El-Etribi K, Nada N, El-Metwally M. The role and limitations of mammary ductoscope in management of pathologic nipple discharge. Breast J. 2008;14(5):442-449. doi:10.1111/j.15244741.2008.00620.x [doi]

45. Zielinski J, Jaworski R, Irga-Jaworska N, Haponiuk I, Jaskiewicz J. The significance of ductoscopy of mammary ducts in the diagnostics of breast neoplasms. Wideochirurgia i inne Tech maloinwazyjne $=$ Videosurgery other miniinvasive Tech. 2015;10(1):79-86. doi:10.5114/wiitm.2014.46823 [doi]

46. Waaijer L, Simons JM, Borel Rinkes IH, van Diest PJ, Verkooijen HM, Witkamp AJ. Systematic review and meta-analysis of the diagnostic accuracy of ductoscopy in patients with pathological nipple discharge. Br J Surg. 2016;103(6):632-643. doi:10.1002/bjs.10125 [doi]

47. Filipe MD, Patuleia SIS, de Jong VMT, Vriens MR, van Diest PJ, Witkamp AJ. A network meta-analysis for the diagnostic approach to pathological nipple discharge. Clin Breast Cancer. Published online June 2020. doi:10.1016/j.clbc.2020.05.015

48. Chhajed PN, Shibuya K, Hoshino H, et al. A comparison of video and autofluorescence bronchoscopy in patients at high risk of lung cancer. Eur Respir J. 2005;25(6):951-955. doi:25/6/951 [pii]

49. Singh R, Mei SC, Sethi S. Advanced endoscopic imaging in Barrett's oesophagus: a review on current practice. World J Gastroenterol. 2011;17(38):4271-4276. doi:10.3748/wjg.v17.i38.4271 [doi] 
50. Moriichi K, Fujiya M, Sato R, et al. Back-to-back comparison of auto-fluorescence imaging (AFI) versus high resolution white light colonoscopy for adenoma detection. BMC Gastroenterol. 2012;12:75. doi:10.1186/1471-230X-12-75 [doi]

51. Ogihara T, Watanabe H, Namihisa A, Kobayashi O, Miwa H, Sato N. Clinical experience using a real time autofluorescence endoscopy system in the gastrointestinal tract. Diagn Ther Endosc. 1999;5(2):119124. doi:10.1155/DTE.5.119 [doi]

52. Thakur A, Gao L, Ren H, Yang T, Chen T, Chen M. Descriptive data on cancerous lung lesions detected by auto-fluorescence bronchoscope: A five-year study. Ann Thorac Med. 2012;7(1):21-25. doi:10.4103/1817-1737.91559 [doi]

53. de Boorder T, Waaijer L, van Diest PJ, Witkamp AJ. Ex vivo feasibility study of endoscopic intraductal laser ablation of the breast. Lasers Surg Med. 2018;50(2):137-142. doi:10.1002/1sm.22745 


\section{Chapter 8}

\section{Ductoscopy for pathological nipple discharge \\ (7 years later)}

M.D. Filipe

S. Makineli

Paul J. van Diest

M.R. Vriens

A.J. Witkamp 


\section{Which technique?}

Ductoscopy is a micro-endoscopic technique that allows real-time visualization of the milk ducts of the breast in the outpatient clinic. Ductoscopy is performed under local anesthesia in women with pathological nipple discharge (PND) in order to detect any intraductal abnormalities. These abnormalities causing PND can be biopsied and removed during ductoscopy. (1) This is important, since PND is associated with one of the first signs of breast cancer.

\section{What is known about effectiveness and side effects?}

Ductoscopy is a diagnostic tool (particularly for the exclusion of malignancy) and also a therapeutic tool to detect and, sometimes remove, the lesion causing PND.

A randomized study from the Royal Marsden Hospital published in 2018 showed that ductoscopy is as sensitive in detecting malignancy in patients with PND as traditional surgery involving the removal of the affected milk duct. (2) We performed a meta-analysis of international studies comparing ductoscopy with MRI, which showed that the pooled sensitivity (i.e., the probability of finding an intraductal abnormality) of ductoscopy and MRI did not differ in a statistically significant manner. However, the pooled specificity (i.e., actually being able to distinguish a found abnormality into benign or malignant) was significantly higher with ductoscopy. The diagnostic accuracy (i.e., how often a test is true positive and true negative compared to the total patients tested) was also significantly higher in ductoscopy (95\%) compared with MRI (83\%) for the detection of breast cancer in patients with nipple discharge without suspicion of malignancy.(3)

A retrospective cohort study of our own center has shown that adding ductoscopy to the diagnostic pathway can prevent invasive surgery in up to $2 / 3$ of women with PND without radiological (echography and/or mammography) suspicion for malignancy.(4) The number of side effects after ductoscopy is limited. The only documented side effects are mastitis $(<1 \%)$ and mild post-procedural pain $(2.5 \%) .(4)$

\section{Has the technique proven to be cost-effective?}

Since ductoscopy has a much lower false positive rate than an MRI (higher specificity in combination with a relatively low incidence of malignancy in patients with nipple discharge), patients undergoing ductoscopy are less likely to undergo the expensive follow-up examinations (3) Moreover, in 2/3 of the patients with PND do not undergo traditional surgery, which is also costly.(4) This means that ductoscopy saves costs in both the diagnosis and treatment of PND.(3) 


\section{Did ductoscopy meet the expectations?}

It was expected that the implantation of ductoscopy to the diagnostic pathway in patients would reduce the number of surgeries in women with PND without radiological suspicion for malignancy.(1) As shown above, adding ductoscopy to the diagnostic pathway for PND is safe, cost-effective and can prevent invasive duct excision surgery in $2 / 3$ of the women.(4)

Whether ductoscopy can also play a role in breast cancer screening in women at an increased risk is still a subject of research.

\section{What indications are there now for ductoscopy?}

Since 2020, ductoscopy has been included in the Breast Cancer guideline as a possible treatment modality in the diagnostic process for PND (spontaneous, bloody, single-sided discharge from 1 milk duct) without suspicion of malignancy on mammography and ultrasound. Negotiations are currently underway with the Zorg Instituut Nederland about coverage of ductoscopy in the basic health insurance package for patients with PND.

\section{Where in the Netherlands?}

Ductoscopy is currently only performed in the UMCU. However, it is expected that ductoscopy will be available in more clinics as a once it is implemented in health insurance 


\section{References}

1. Waaijer L, van Diest P, van der Pol CC, Verolme B, Hennink A, Witkamp AJ. Ductoscopy for pathologic nipple discharge. Ned Tijdschr Geneeskd. 2013;157(29):A6358.

2. Gui G, Agusti A, Twelves D, Tang S, Kabir M, Montgomery C, et al. INTEND II randomized clinical trial of intraoperative duct endoscopy in pathological nipple discharge. Br J Surg. 2018 Nov;105(12):158390 .

3. Filipe, M.D.; Patuleia, S.I.S.; Vriens, M.R.; van Diest, P.J.; Witkamp AJ. Meta-analysis and costeffectiveness of ductoscopy, duct excision surgery and MRI for the diagnosis and treatment of patients with pathological nipple discharge.

4. Filipe MD, Waaijer L, van der Pol CC, van Diest P, Witkamp AJ. Interventional ductoscopy as an alternative for major duct excision or microdochectomy in women suffering pathological nipple discharge: a single centre experience. Clin Breast Cancer. 2020 Jan 27. 
Ductoscopy for pathological nipple discharge (7 years later) 

Robotic nipple Sparing mastectomy complication rate compared to traditional nipple sparing mastectomy: a systematic review and meta-analysis.

M.D. Filipe E. de Bock E.L. Postma

O.W. Bastian P.P.A. Schellekens M.R. Vriens

A.J. Witkamp M.C. Richir 


\section{Abstract}

Introduction: Breast cancer is worldwide the most common cause of cancer in women and causes the second most common cancer related death. Nipple sparing mastectomy (NSM) is commonly used in therapeutic and prophylactic settings. Furthermore, (preventive) mastectomies are, besides complications, also associated with psychological and cosmetic consequences. Robotic NSM (RNSM) allows for better visualization of the planes and reducing the invasiveness. The aim of this study was to compare the postoperative complication rate of RNSM to NSM.

Methods: A systematic search was performed on all (R)NSM articles. The primary outcome was determining the overall postoperative complication rate of traditional NSM and RNSM. Secondary outcomes were comparing the specific postoperative complication rates: implant loss, hematoma, (flap)necrosis, infection, and seroma.

Results: 49 studies containing 13,886 cases of (R)NSM were included. No statistically significant differences were found regarding postoperative complications (RNSM 3.9 $\%$, NSM 7.0\%, p = 0.070), postoperative implant loss (RNSM 4.1\%, NSM 3.2\%, p $=0.523)$, hematomas (RNSM 4.3\%, NSM 2.0\%, p = 0.059), necrosis (RNSM 4.3\%, NSM 7.4\%, p = 0.230), infection (RNSM 8.3\%, NSM 4.0\%, p = 0.054) or seromas (RNSM $3.0 \%$, NSM $2.0 \%, \mathrm{p}=0.421$ ).

Conclusion: Overall, there are no statistically significant differences in complication rates between NSM and RNSM. 


\section{Introduction}

Breast cancer is the most common type of cancer in women and the second most common cause of death due to cancer in women worldwide. ${ }^{1}$ There are approximately 17.000 new cases of breast cancer in the Netherlands every year. In addition, over 3.000 women of the Dutch population annually die due to breast cancer. ${ }^{2}$ Hereditary breast cancer accounts for up to $5-10 \%$ of all breast cancers. Two high-penetrance genes (BRCA1 and BRCA2) are responsible for about $16 \%$ of the familial risk of breast cancers and associated with a $60-80 \%$ lifetime risk of developing breast cancer. ${ }^{3,4}$

Surgical resection of the primary tumor is the treatment of choice in patients with new onset breast cancer. Tumor stage and molecular characteristics determine the type of surgery. Most patients are treated by breast conserving surgery followed by radiation therapy (breast conserving therapy, BCT) or mastectomy with or without breast reconstruction. ${ }^{5,6}$

Currently, the ultimate prevention in women with hereditary breast cancer is bilateral prophylactic mastectomy. ${ }^{7}$ Consequently, this means that $20-40 \%$ of these patients undergo mastectomies without signs of malignancy. Unfortunately, a part of these patients will develop complications or experience poor cosmetic results and carry a significant psychological burden. ${ }^{8,9}$

Nipple sparing mastectomy (NSM) was initially reserved for the prophylactic treatment of women with a high risk of developing breast cancer. ${ }^{10}$ However, NSM has been increasingly used therapeutically for breast cancer where the nipple-areolar complex is not involved. ${ }^{11-14}$ One of the most important challenges of nipple-sparing mastectomy is achieving adequate exposure to perform precise dissection in areas that are remote from the skin incision. ${ }^{15}$

Robotic nipple-sparing mastectomy (RNSM) is a relatively new technique that allows for better visualization of tissue planes and expose tissue that is challenging to reach with traditional nipple-sparing mastectomy techniques. ${ }^{16-18}$ Previous research has not only demonstrated the feasibility and safety of RNSM, but also that RNSM has a steep learning curve. ${ }^{16,19}$ However, there are currently no studies comparing the complication rate of RNSM to NSM. Therefore, the aim of this study is to compare postoperative complications of patients undergoing traditional NSM to RNSM followed by immediate breast reconstruction. 


\section{Materials and Methods}

This systematic review and meta-analysis was performed according to the guidelines of the requirements of the PRISMA Checklist for meta-analysis. ${ }^{20}$ A systematic literature search was performed in the PubMed, Embase and Cochrane Library databases. The search strategy was performed on all index tests (NSM and RNSM) and their synonyms. The full electronic search strategy can be found in the supplementary data (Appendix 1). After removal of duplicates, two authors (MF, EB) independently screened articles by title and abstract. The two authors discussed discordant judgments until consensus was reached. The full articles were independently screened for eligibility based on predefined inclusion and exclusion criteria.

\section{Selection of studies}

Full texts were retrieved for studies that evaluated (robot) nipple sparing mastectomy, reported original data and were written in English.

6. Participants: patients undergoing therapeutic or prophylactic (R)NSM with immediate breast reconstruction (IBR).

7. Intervention: (R)NSM.

8. Outcome: postoperative complications (implant loss, hematoma, necrosis, infection or seroma).

Studies were excluded from systematic review based on the following criteria:

4. Not possible to determine whether patients had immediate reconstruction.

5. Non-robotic endoscopic NSM and/or reconstruction.

6. Intra-operative radiotherapy.

7. Case report, review and conference abstracts.

\section{Risk of bias}

The ROBINS-I Tool was used to evaluate the quality of each eligible study. ${ }^{21}$ The entire scale constituted seven domains for the risk of bias; confounding, selection of participants, classification of interventions, deviations from intended interventions, missing data, measurement of outcomes and selection of the reported result. Each domain was judged for three levels of bias: low risk, intermediate/unclear risk, or high risk of bias. Full assessment criteria can be found in the supplementary data (Appendix 2). 


\section{Statistical analysis}

Pairwise meta-analysis was performed to compare complication rates with $95 \%$ confidence intervals (CI) of RNSM to NSM in studies. Pooled postoperative complication rates were determined using random effects models. P-values under 0.05 were considered statistically significant.

All calculations were performed using RStudio 1.2.5001 (with $\mathrm{R}$ version: x64 3.6.3). Additionally, statistical packages meta, mada, metafor, gemtc, mvmeta and were used for all computations of the meta-analyses. Visualization of plots was done using the ggplot2 package. 


\section{Results}

1,116 citations were identified by the search and, after removing duplicates, 95 potentially eligible articles were retrieved in full text (Figure 1). Overall, 13,886 (R)NSM were performed in 49 studies with an average of 294.6 participants per study.
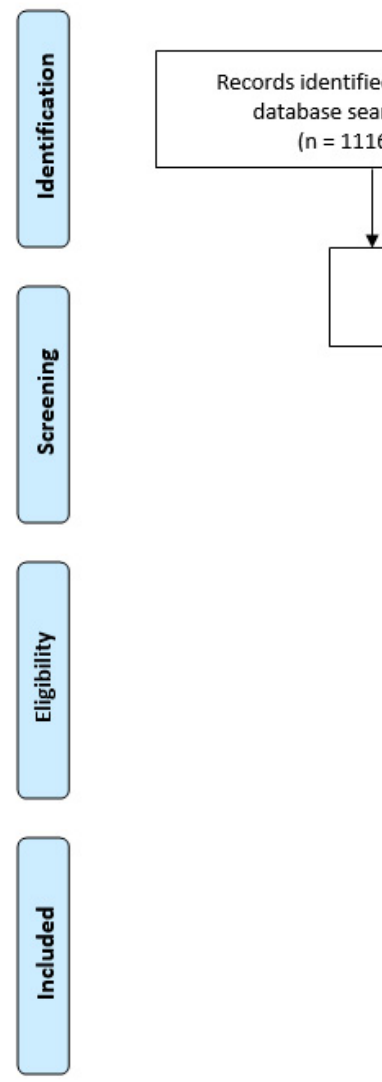

Records after duplicates removed

Additional records identified through other sources $(n=1116$ ) $(\mathrm{n}=0)$

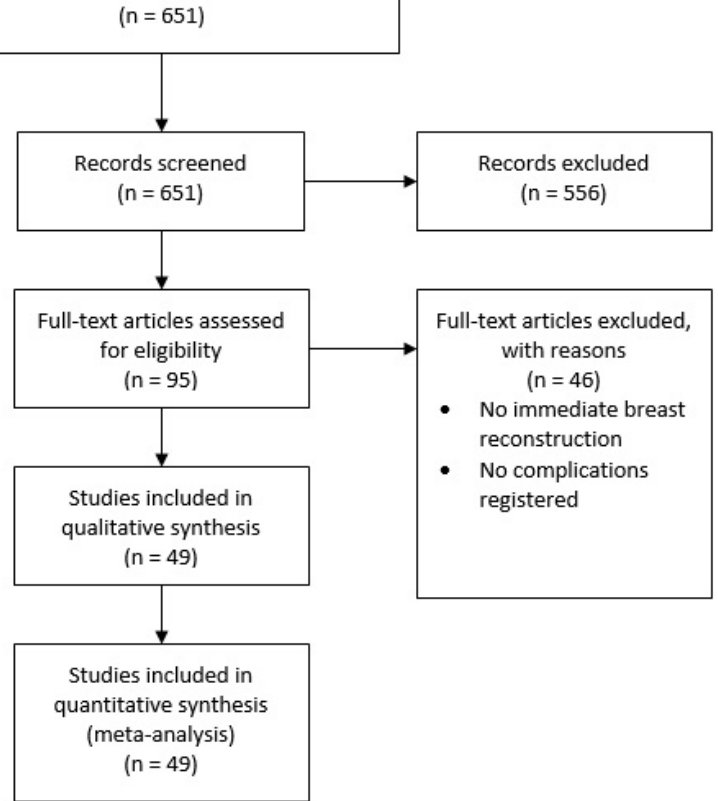

Figure 1. Flow chart showing literature search and study selection with 49 relevant studies ultimately enrolled in this meta-analysis on the complication rate of (robotic) nipple sparing mastectomy. 
Supplementary Table 1 shows the studies included in the analysis and their characteristics. Seven studies described postoperative complications of RNSM and 42 studies described the postoperative complications of NSM. In total, 13 out of 225 mastectomies $(3.9 \%)$ developed postoperative complications in RNSM while 1,056 out of 13,661 NSM (7.0\%) developed postoperative complications. This difference was not statistically significant ( $\mathrm{p}$ $=0.070)$ (Figure 2 ).

Table 1. Pooled complication rates of (R)NSM.

\begin{tabular}{llll}
\hline Parameter & RNSM & NSM & p-value \\
\hline Total complications, \% (95\% CI) & $3.9 \%(0.8-6.9 \%)$ & $7.0 \%(5.6-8.4 \%)$ & 0.070 \\
Implant loss, \% (95\% CI) & $4.1 \%(1.9-8.7 \%)$ & $3.2 \%(2.4-4.2 \%)$ & 0.523 \\
Hematoma, \% (95\% CI) & $4.3 \%(2.0-9.1 \%)$ & $2.0 \%(1.7-2.4 \%)$ & 0.059 \\
Necrosis, \% (95\% CI) & $4.3 \%(1.8-10.0 \%)$ & $7.4 \%(5.8-9.3 \%)$ & 0.230 \\
Infection, \% (95\% CI) & $8.3 \%(4.2-15.8 \%)$ & $4.0 \%(3.0-5.3 \%)$ & 0.054 \\
Seroma, \% (95\% CI) & $3.0 \%(1.3-7.1 \%)$ & $2.0 \%(1.3-3.1 \%)$ & 0.421 \\
\hline
\end{tabular}

(R) NSM = (robotic) nipple sparing mastectomy, CI = confidence interval. 


\section{Study}

'Type of surgery' = NSM

Chan et al. 2017

Colwell et al. 2017

De Vita et al. 2017

Dull et al. 2017

Frey et al. 2017

Frey et al. 2017

Frey et al. 2017

Frey et al. 2017

Hashem et al. 2017

Lago et al. 2017

Mesdag et al. 2017

Murphy et al. 2017

Rancati et al. 2017

Sbitany et al. 2017

Venturi et al. 2017

Casella et al. 2018

de la Parra Marquez et al. 2018

Frey et al. 2018

Frey et al. 2018

Odom et al. 2018

Pek et al. 2018

Qureshi et al. 2018

Roh et al. 2018

de Vita et al. 2019

Ito et al. 2019

$\mathrm{Kim}$ et al. 2019

$\mathrm{Ng}$ et al. 2019

Pallara et al. 2019

Quinn et al. 2019

Reitsamer et al. 2019

Salibian et al. 2019

Tasoulis et al. 2019

Yang et al. 2019

Young et al. 2019

Chan et al. 2020

Moon et al. 2020

Park et al. 2020

Salibian et al. 2020

Seki et al. 2020

Valero et al. 2020

Wang et al. 2020

Willey et al. 2020

Random effects model

Heterogeneity: $I^{2}=92 \%, \tau^{2}=0.0017, p<0.01$

'Type of surgery' = RNSM

Toesca et al. 2017

Lai et al. 2018

Sarfati et al. 2018

Houvenaeghel et al. 2019

Lai et al. 2019

Park et al. 2019

Lai et al. 2020

Random effects model

Heterogeneity: $I^{2}=18 \%, \tau^{2}=0.0003, p=0.30$

Random effects model

Heterogeneity: $I^{2}=90 \%, \tau^{2}=0.0017, p<0.0$

Residual heterogeneity: $I^{2}=90 \%, p<0.01$
Number of
complications Total $\begin{gathered}\text { Events per 10u } \\ \text { observations }\end{gathered}$

Percentage (\%) $\quad$ 95\%-Cl Weight

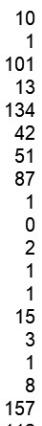

103

132

$\begin{array}{rr}101 & 2023 \\ 13 & 197\end{array}$

$\begin{array}{ll}134 & 1028\end{array}$

$42 \quad 543$

$\begin{array}{ll}51 & 420 \\ 87 & 798\end{array}$

$\begin{array}{ll:l}1 & 55+ & + \\ 0 & 69+ & \end{array}$

263

19

30

32

32

40

$\begin{array}{ll}8 & 1407 \\ 7 & 1207\end{array}$

11

87

$16 \quad 382$

$14 \quad 145$

0
17

$17 \quad 123$

2
29

$\begin{array}{ll}29 & 1 \\ 19 & 10\end{array}$

443

$\begin{array}{rr}29 & 200 \\ 63 & 1045\end{array}$

$\begin{array}{rr}63 & 1045 \\ 8 & 82\end{array}$

$0 \quad 130$

$55 \quad 1301$

$\begin{array}{rr}0 & 61 \\ 22 & 214 \\ 17 & 114\end{array}$

$17 \quad 114$

$\begin{array}{rr}2 & 44 \\ 10 & 181\end{array}$

$\begin{array}{ll}10 & 181 \\ 28 & 777\end{array}$

$13 \quad 217$

$5 \quad 140$

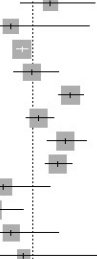

\begin{tabular}{l}
+ \\
+1 \\
\hline+
\end{tabular}

2

$+$

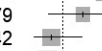

$+$

1

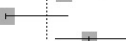

$+$
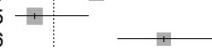

1

$+$
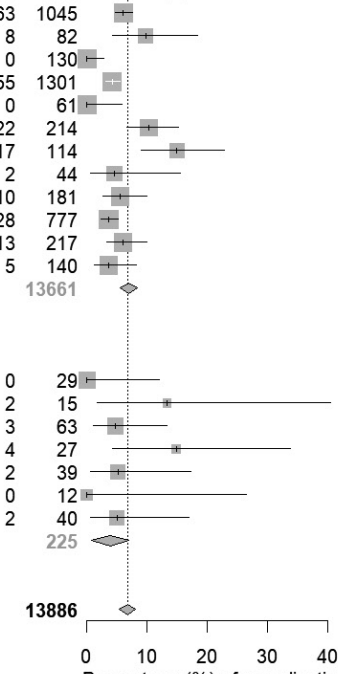

Percentage (\%) of complications
$9.71[4.75 ; 17.13] \quad 1.8 \%$

$3.12[0.08 ; 16.22] \quad 1.8 \%$

$4.99 \quad[4.08 ; 6.03] \quad 2.7 \%$

6.60 [3.56; 11.02] $2.3 \%$

$13.04[11.04 ; 15.25] \quad 2.6 \%$

$7.73[5.63 ; 10.31] \quad 2.6 \%$

$12.14[9.18 ; 15.66] \quad 2.4 \%$

$10.90[8.82 ; 13.27] \quad 2.6 \%$

$1.82[0.05 ; 9.72] \quad 2.3 \%$

$0.00 \quad[0.00 ; 5.21] \quad 2.6 \%$

$3.17[0.39 ; 11.00] \quad 2.1 \%$

$5.26[0.13 ; 26.03] \quad 1.1 \%$

$3.33[0.08 ; 17.22] \quad 1.7 \%$

$5.56 \quad[3.14 ; 9.00] \quad 2.5 \%$

$1.09[0.03 ; 5.91] \quad 2.6 \%$

$5.71[2.50 ; 10.95] \quad 2.2 \%$

$13.01[11.16 ; 15.04] \quad 2.6 \%$

10.13 [ $4.47 ; 18.98] \quad 1.6 \%$

$4.93 \quad[2.00 ; 9.89] \quad 2.3 \%$

$4.19[2.41 ; 6.71] \quad 26 \%$

$9.66[5.38 ; 15.67] \quad 2.0 \%$

$0.00[0.00 ; 10.28] \quad 2.2 \%$

$13.82[8.26 ; 21.20] \quad 1.7 \%$

$3.64[0.44 ; 12.53] \quad 2.0 \%$

$17.92[11.15 ; 26.57] \quad 1.5 \%$

$9.30[2.59 ; 22.14] \quad 1.3 \%$

$14.50[9.93 ; 20.16] \quad 2.0 \%$

$6.03[4.66 ; 7.65] \quad 2.7 \%$

$9.76[4.31 ; 18.32] \quad 1.7 \%$

$0.00 \quad[0.00 ; 2.80] \quad 2.7 \%$

$4.23[3.20 ; 5.47] \quad 2.7 \%$

0.00 [0.00; 5.87] $2.6 \%$

$10.28[6.56 ; 15.15] \quad 2.2 \%$

$14.91[8.93 ; 22.80] \quad 1.7 \%$

$4.55[0.56 ; 15.47] \quad 1.7 \%$

$5.52[2.68 ; 9.93] \quad 2.4 \%$

$3.60[2.41 ; 5.17] \quad 2.7 \%$

$5.99[3.23 ; 10.03] \quad 2.4 \%$

$3.57 \quad[1.17 ; 8.14]$

$6.97[5.57 ; 8.38] \quad 90.6 \%$

$0.00 \quad[0.00 ; 11.94] \quad 2.1 \%$

$13.33[1.66 ; 40.46] \quad 0.5 \%$

$4.76[0.99 ; 13.29] \quad 1.9 \%$

$14.81[4.19 ; 33.73] \quad 0.7 \%$

$5.13[0.63 ; 17.32] \quad 1.6 \%$

$0.00[0.00 ; 26.46] \quad 1.0 \%$

$5.00[0.61 ; 16.92] \quad 1.6 \%$

$3.87[0.83 ; 6.92] \quad 9.4 \%$

$6.74[5.41 ; 8.07] 100.0 \%$
$9.38[1.98 ; 25.02] \quad 1.1 \%$

$13.84[11.54 ; 16.42] \quad 2.5 \%$

$25.00[17.43 ; 33.89] \quad 1.4 \%$

Figure 2. Complication rates. (R)NSM = (robotic) nipple sparing mastectomy, $\mathrm{CI}=$ confidence interval.

\section{Different postoperative complications}

Detailed meta-analysis of the different complications of each study can be found in supplementary Figures 1 to 5. Post mastectomy implant removal occurred in $4.1 \%$ of RNSM and in 3.2\% in NSM. This difference was not statistically significant ( $\mathrm{p}=0.523)$. Furthermore, post mastectomy hematoma occurred more often in RNSM (4.3\%) than 
in NSM (2.0\%) but this difference was not statistically significant $(\mathrm{p}=0.059)$. Moreover, post mastectomy necrosis and seroma occurred in respectively $4.3 \%$ and $3.0 \%$ in RNSM and $7.4 \%$ and $2.0 \%$ in NSM. These differences were not statistically significant. Finally, postoperative mastectomy infection occurred more often in RNSM (8.3\%) than in NSM $(4.0 \%)$ but this was not statistically significant $(\mathrm{p}=0.054)$ (Table 1$)$.

\section{Risk of bias}

The result of the ROBINS-I Tool revealed that all the included studies were of sufficient quality. This was for risks of bias domains and applicability domains (Figure 3). Risk assessment of every study can be found in Appendix 4 .

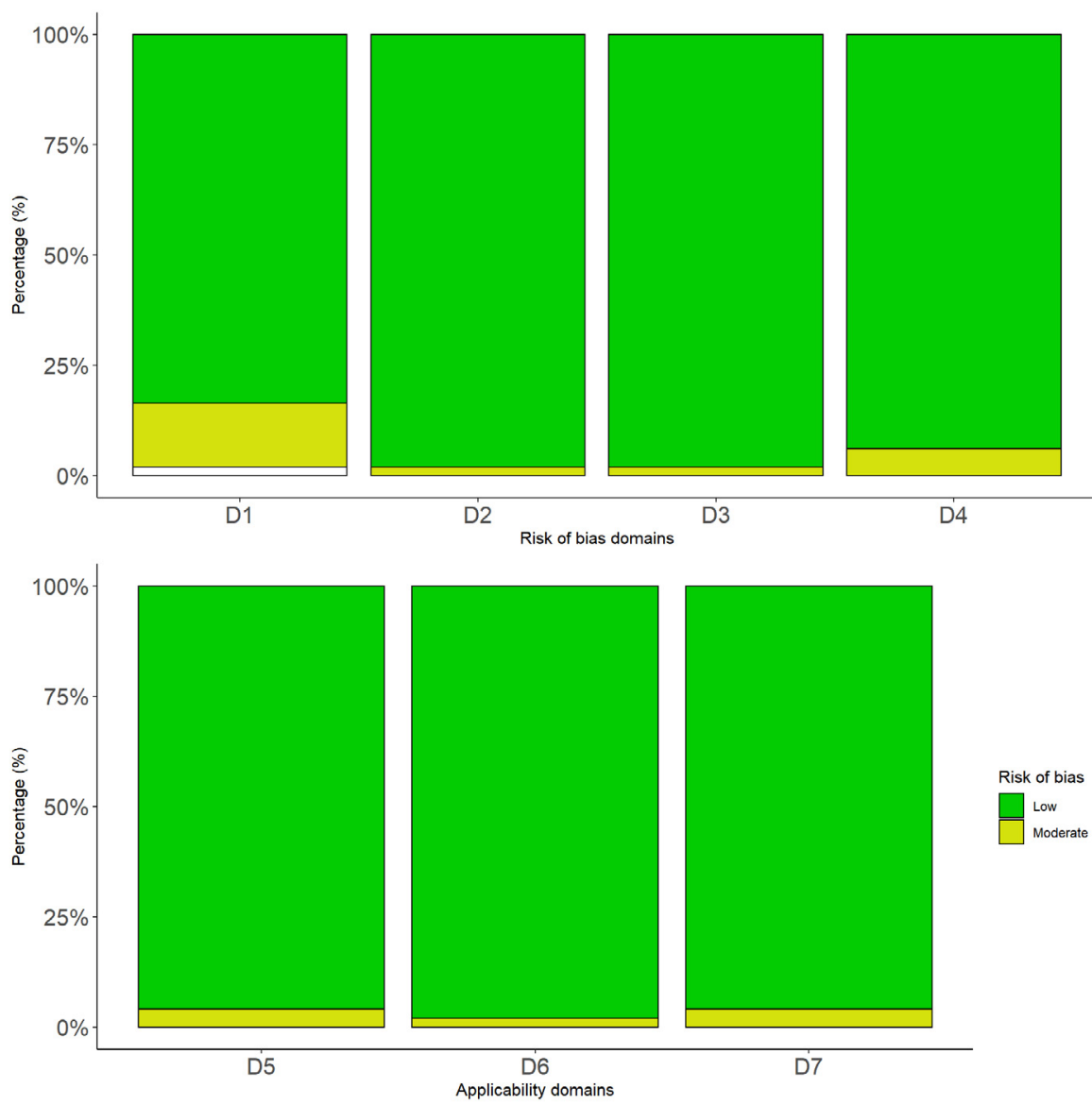

Figure 3. Summary of the risks of bias and applicability domains. D1= Bias due to confounding D2 = Bias in selection of participants into the study; D3 = Bias in classification of interventions; D4 = Bias due to deviations from intended interventions; D5 = Bias due to missing data; D6 = Bias in measurements of outcomes; D7 = Bias in selection of the reported result. 


\section{Discussion}

This meta-analysis, including 49 studies, is the first study to compare the complication rate of RNSM to NSM in patients undergoing prophylactic or therapeutic mastectomy. The current study shows that patients undergoing RNSM do not have an increased risk of developing postoperative complications when compared to NSM.

Approximately $40 \%$ of patients with invasive breast cancer and $30 \%$ of patients with ductal carcinoma in situ (DCIS) undergo mastectomy. ${ }^{22}$ Additionally, there are many BRCA1 or BRCA2 gene mutation carriers that undergo prophylactic mastectomies. As mastectomies are accompanied by complications along with serious cosmetic and psychological consequences. ${ }^{8,9}$ For these patients, it is essential to keep the surgical intervention as minimally invasive as possible, while preserving the (oncological) safety. RNSM has shown to be feasible and safe, both as a therapeutic and a prophylactic treatment. ${ }^{19,23}$ In order to obtain information to provide a reliable insight into the postoperative complications between NSM and RNSM procedures, a literature search was performed. Overall complication rate was 3.9\% after RNSM and 7.0\% after NSM. This difference was not statistically different. Considering individual complications, patients did not appear to have an increased risk of postoperative implant loss or developing hematomas, infections, seromas or necrosis due to RNSM.

While there are no differences in complications between traditional NSM and RNSM, RNSM does offer certain advantages. Robotic surgery in general provides smaller incisions compared to open surgery. ${ }^{24}$ Furthermore, RNSM allows for better visualization of the planes and exposes tissue that is challenging to reach with traditional nipple-sparing mastectomy techniques. ${ }^{16-18}$ Another advantage of the scopic nature of the RNSM could be that this technique allows enhanced imaging techniques that could detect (pre)cancerous breast cancer lesions. One of these techniques is narrow band imaging (NBI). NBI is a well-established technique used during colonoscopy, cystoscopy and bronchoscopy to detect (pre)cancerous lesions of epithelial origin. ${ }^{25-27}$ Therefore, it is reasonable to assume that NBI might be useful since (pre)malignancy is known to show different patterns of vascularization compared to healthy breast tissue. ${ }^{28,29}$ Furthermore, (pre)malignant epithelial lesions show an aberrant pattern under fluorescent light by which they become detectable. This technique is already used extensively in order to help identify pathologies within the airways, larynx and colon. ${ }^{30-32}$ Moreover, studies have also shown promising results of (auto) fluorescence for the detection of (pre)cancerous lesions of the breast. ${ }^{33,34}$ Consequently, enhanced imaging techniques, such as NBI and (auto) fluorescence, combined with robotic surgery could improve margin determination in patients undergoing breast cancer surgery. Furthermore, another advantage of robotic surgery is that it provides better ergonomics for the surgeon when compared to traditional surgery. ${ }^{35}$ A possible downside of robotic breast surgery, as with many other types of robotic surgery, is that the preparations (docking and positioning of the patient) and the 
procedure itself takes longer. ${ }^{16}$ The longer operation time results, along with the higher material costs, in an overall more expensive procedure.

A recent systematic review also concluded that RNSM is safe to use with acceptable shortterm outcomes. ${ }^{36}$ However, the current study quantifies (by means of a meta-analysis) the risk of postoperative complications in patients undergoing RNSM and compares said risk to traditional mastectomy.

This study has some limitations. The number of patients in the studies describing RNSM are relatively low compared to traditional NSM. This can be attributed to the fact that RNSM is a relatively new technique. Furthermore, the current study did not describe which percentage of the mastectomies were prophylactic or therapeutic. This might have an effect on the risk of postoperative complications since neo-adjuvant therapy increases the risk of postoperative complications. ${ }^{37}$ Although RNSM has great potential, additional prospective research is warranted to further determine oncological safety, longterm postoperative complications and patient reported outcomes in patients undergoing RNSM. Currently, in most countries RNSM is off-label. Therefore, the previously proposed additional research and the results of the current study could help making RNSM a recommended viable option for women requiring mastectomy.

In conclusion, this study shows that there is no significant difference in postoperative complication rate of RNSM compared to traditional NSM. Therefore, RNSM can be used safely in patients that require prophylactic or therapeutic mastectomy. 


\section{References}

1. Siegel RL, Miller KD, Jemal A. Cancer statistics, 2018. CA Cancer J Clin. 2018;68(1):7-30. doi:10.3322/ caac.21442 [doi]

2. Vondeling GT, Menezes GL, Dvortsin EP, et al. Burden of early, advanced and metastatic breast cancer in The Netherlands. BMC Cancer. 2018;18(1):262-263. doi:10.1186/s12885-018-4158-3 [doi]

3. Ford D, Easton DF, Stratton M, et al. Genetic heterogeneity and penetrance analysis of the BRCA1 and BRCA2 genes in breast cancer families. The Breast Cancer Linkage Consortium. Am J Hum Genet. 1998;62(3):676-689. doi:S0002-9297(07)63848-8 [pii]

4. Tung N, Battelli C, Allen B, et al. Frequency of mutations in individuals with breast cancer referred for BRCA1 and BRCA2 testing using next-generation sequencing with a 25 -gene panel. Cancer. 2015;121(1):25-33. doi:10.1002/cncr.29010 [doi]

5. Hartmann-Johnsen OJ, Karesen R, Schlichting E, Nygard JF. Survival is Better After Breast Conserving Therapy than Mastectomy for Early Stage Breast Cancer: A Registry-Based Follow-up Study of Norwegian Women Primary Operated Between 1998 and 2008. Ann Surg Oncol. 2015;22(12):38363845. doi:10.1245/s10434-015-4441-3 [doi]

6. van Maaren MC, de Munck L, de Bock GH, et al. 10 year survival after breast-conserving surgery plus radiotherapy compared with mastectomy in early breast cancer in the Netherlands: a population-based study. The LancetOncology. 2016;17(8):1158-1170. doi:S1470-2045(16)30067-5 [pii]

7. Casella D, Di Taranto G, Marcasciano M, et al. Nipple-sparing bilateral prophylactic mastectomy and immediate reconstruction with TiLoop $\left({ }^{\circ}\right)$ Bra mesh in BRCA1/2 mutation carriers: A prospective study of long-term and patient reported outcomes using the BREAST-Q. Breast. 2018;39:8-13. https://pubmed. ncbi.nlm.nih.gov/29455110.

8. Kim H, Park S-J, Woo K-J, Bang SI. Comparative Study of Nipple-Areola Complex Position and Patient Satisfaction After Unilateral Mastectomy and Immediate Expander-Implant Reconstruction NippleSparing Mastectomy Versus Skin-Sparing Mastectomy. Aesthetic Plast Surg. 2019;43(2):313-327. https:// pubmed.ncbi.nlm.nih.gov/30746565.

9. Pek W-S, Tan B-K, Ru Ng YY, et al. Immediate breast reconstruction following nipple-sparing mastectomy in an Asian population: Aesthetic outcomes and mitigating nipple-areolar complex necrosis. Arch Plast Surg. 2018;45(3):229-238. https://pubmed.ncbi.nlm.nih.gov/29788682.

10. Newman LA, Kuerer HM, Hunt KK, et al. Prophylactic mastectomy. J Am Coll Surg. 2000;191(3):322330. doi:10.1016/S1072-7515(00)00361-6

11. Mesdag V, Régis C, Tresch E, et al. Nipple sparing mastectomy for breast cancer is associated with high patient satisfaction and safe oncological outcomes. J Gynecol Obstet Hum Reprod. 2017;46(8):637-642. doi:10.1016/j.jogoh.2017.07.003

12. Lago V, Maisto V, Gimenez-Climent J, Vila J, Vazquez C, Estevan R. Nipple-sparing mastectomy as treatment for patients with ductal carcinoma in situ: A 10-year follow-up study. Breast J. 2018;24(3):298303. doi:10.1111/tbj.12947

13. Chan YH-Y, Yau W-M, Cheung PS-Y. Oncological Safety and Technical Feasibility of Nipple-Sparing Mastectomy for Breast Cancer: The Hong Kong Experience. World J Surg. 2018;42(5):1375-1383. doi: $10.1007 /$ s00268-017-4197-y

14. Galimberti V, Vicini E, Corso G, et al. Nipple-sparing and skin-sparing mastectomy: Review of aims, oncological safety and contraindications. The Breast. 2017;34:S82-S84. doi:10.1016/j.breast.2017.06.034

15. Coopey SB, Mitchell SD. Nipple-Sparing Mastectomy: Pitfalls and Challenges. Ann Surg Oncol. 2017;24(10):2863-2868. doi:10.1245/s10434-017-5962-8 
16. Toesca A, Peradze N, Manconi A, et al. Robotic nipple-sparing mastectomy for the treatment of breast cancer: Feasibility and safety study. Breast. 2017;31:51-56. doi:S0960-9776(16)30191-6 [pii]

17. Park HS, Lee J, Lee DW, et al. Robot-assisted Nipple-sparing Mastectomy with Immediate Breast Reconstruction: An Initial Experience. Sci Rep. 2019;9(1):15669. https://pubmed.ncbi.nlm.nih. gov/31666551.

18. Lai H-W, Chen S-T, Lin S-L, et al. Robotic Nipple-Sparing Mastectomy and Immediate Breast Reconstruction with Gel Implant: Technique, Preliminary Results and Patient-Reported Cosmetic Outcome. Ann Surg Oncol. 2019;26(1):42-52. https://pubmed.ncbi.nlm.nih.gov/30109537.

19. Houvenaeghel G, Bannier M, Rua $S$, et al. Breast cancer robotic nipple sparing mastectomy: evaluation of several surgical procedures and learning curve. World J Surg Oncol. 2019;17(1):27-019-1567-y. doi:10.1186/s12957-019-1567-y [doi]

20. Liberati A, Altman DG, Tetzlaff J, et al. The PRISMA statement for reporting systematic reviews and meta-analyses of studies that evaluate healthcare interventions: explanation and elaboration. BMJ. 2009;339(jul21 1):b2700-b2700. doi:10.1136/bmj.b2700

21. Sterne JA, Hernán MA, Reeves BC, et al. ROBINS-I: a tool for assessing risk of bias in non-randomised studies of interventions. BMJ. October 2016:i4919. doi:10.1136/bmj.i4919

22. van Bommel ACM, Spronk PER, Vrancken Peeters M-JTFD, et al. Clinical auditing as an instrument for quality improvement in breast cancer care in the Netherlands: The national NABON Breast Cancer Audit. J Surg Oncol. 2017;115(3):243-249. doi:10.1002/jso.24516

23. Houvenaeghel G, Cohen M, Ribeiro SR, et al. Robotic Nipple-Sparing Mastectomy and Immediate Breast Reconstruction With Robotic Latissimus Dorsi Flap Harvest: Technique and Results. Surg Innov. May 2020:155335062091791. doi:10.1177/1553350620917916

24. van der Sluis PC, Ruurda JP, van der Horst S, et al. Robot-assisted minimally invasive thoraco-laparoscopic esophagectomy versus open transthoracic esophagectomy for resectable esophageal cancer, a randomized controlled trial (ROBOT trial). Trials. 2012;13(1):230. doi:10.1186/1745-6215-13-230

25. Singh R, Mei SC, Sethi S. Advanced endoscopic imaging in Barrett's oesophagus: a review on current practice. World J Gastroenterol. 2011;17(38):4271-4276. doi:10.3748/wjg.v17.i38.4271 [doi]

26. Jichlinski P, Lovisa B. High magnification cystoscopy in the primary diagnosis of bladder tumors. Curr Opin Urol. 2011;21(5):398-402. doi:10.1097/MOU.0b013e32834956ad [doi]

27. Tanaka S, Sano Y. Aim to unify the narrow band imaging (NBI) magnifying classification for colorectal tumors: current status in Japan from a summary of the consensus symposium in the 79th Annual Meeting of the Japan Gastroenterological Endoscopy Society. Dig Endosc. 2011;23 Suppl 1:131-139. doi:10.1111/j.1443-1661.2011.01106.x [doi]

28. Gadre SA, Perkins GH, Sahin AA, Sneige N, Deavers MT, Middleton LP. Neovascularization in mucinous ductal carcinoma in situ suggests an alternative pathway for invasion. Histopathology. 2008;53(5):545553. doi:10.1111/j.1365-2559.2008.03152.x [doi]

29. Li L, Wang K, Sun X, et al. Parameters of dynamic contrast-enhanced MRI as imaging markers for angiogenesis and proliferation in human breast cancer. Med Sci Monit. 2015;21:376-382. doi:10.12659/ MSM.892534 [doi]

30. Ogihara T, Watanabe H, Namihisa A, Kobayashi O, Miwa H, Sato N. Clinical experience using a real time autofluorescence endoscopy system in the gastrointestinal tract. Diagn Ther Endosc. 1999;5(2):119124. doi:10.1155/DTE.5.119 [doi]

31. Ikeda N, Honda H, Hayashi A, et al. Early detection of bronchial lesions using newly developed videoendoscopy-based autofluorescence bronchoscopy. Lung Cancer. 2006;52(1):21-27. doi:S0169-5002(06)00018-3 [pii] 
32. van der Heijden EH, Hoefsloot W, van Hees HW, Schuurbiers OC. High definition bronchoscopy: a randomized exploratory study of diagnostic value compared to standard white light bronchoscopy and autofluorescence bronchoscopy. Respir Res. 2015;16:33-37. doi:10.1186/s12931-015-0193-7 [doi]

33. Douplik A, Leong WL, Easson AM, Done S, Netchev G, Wilson BC. Feasibility study of autofluorescence mammary ductoscopy. J Biomed Opt. 2009;14(4):44036. doi:10.1117/1.3210773 [doi]

34. Waaijer L, Filipe MD, Simons J, et al. Detection of breast cancer precursor lesions by autofluorescence ductoscopy. Breast Cancer. July 2020. doi:10.1007/s12282-020-01136-6

35. Lee GI, Lee MR, Green I, Allaf M, Marohn MR. Surgeons' physical discomfort and symptoms during robotic surgery: a comprehensive ergonomic survey study. Surg Endosc. 2017;31(4):1697-1706. doi:10.1007/s00464-016-5160-y

36. Angarita FA, Castelo M, Englesakis M, McCready DR, Cil TD. Robot-assisted nipple-sparing mastectomy: systematic review. Br J Surg. August 2020. doi:10.1002/bjs.11837

37. Zhang L, Jin K, Wang X, et al. The Impact of Radiotherapy on Reoperation Rates in Patients Undergoing Mastectomy and Breast Reconstruction. Ann Surg Oncol. 2019;26(4):961-968. doi:10.1245/s10434018-07135-4

38. Frey JD, Choi M, Salibian AA, Karp NS. Comparison of Outcomes with Tissue Expander, Immediate Implant, and Autologous Breast Reconstruction in Greater Than 1000 Nipple-Sparing Mastectomies. Plast Reconstr Surg. 2017;139(6):1300-1310. doi:10.1097/PRS.0000000000003340

39. Rancati AO, Angrigiani CH, Hammond DC, et al. Direct to Implant Reconstruction in Nipple Sparing Mastectomy. Plast Reconstr Surg - Glob Open. 2017;5(6):e1369. doi:10.1097/GOX.0000000000001369

40. Frey JD, Alperovich M, Levine JP, Choi M, Karp NS. Does Smoking History Confer a Higher Risk for Reconstructive Complications in Nipple-Sparing Mastectomy? Breast J. 2017;23(4):415-420. doi:10.1111/tbj. 12760

41. Frey JD, Salibian AA, Choi M, Karp NS. Mastectomy Flap Thickness and Complications in Nipple-Sparing Mastectomy. Plast Reconstr Surg - Glob Open. 2017;5(8):e1439. doi:10.1097/GOX.0000000000001439

42. Dull B, Conant L, Myckatyn T, Tenenbaum M, Cyr A, Margenthaler JA. Nipple-sparing mastectomies: Clinical outcomes from a single academic institution. Mol Clin Oncol. 2017;6(5):737-742. doi:10.3892/ mco.2017.1208

43. Murphy BL, Boughey JC, Hieken TJ. Nipple-sparing Mastectomy for the Management of Recurrent Breast Cancer. Clin Breast Cancer. 2017;17(4):e209-e213. doi:10.1016/j.clbc.2016.10.011

44. Colwell AS, Taylor E, Specht M, Orringer JS. Optimizing Nipple Position following Nipple-Sparing Mastectomy. Plast Reconstr Surg - Glob Open. 2017;5(9):e1490. doi:10.1097/GOX.0000000000001490

45. De Vita R, Zoccali G, Buccheri EM, Costantini M, Botti C, Pozzi M. Outcome Evaluation after 2023 Nipple-Sparing Mastectomies. Plast Reconstr Surg. 2017;139(2):335e-347e. doi:10.1097/ PRS.0000000000003027

46. Sbitany H, Piper M, Lentz R. Prepectoral Breast Reconstruction. Plast Reconstr Surg. 2017;140(3):432443. doi:10.1097/PRS.0000000000003627

47. Venturi ML, Mesbahi AN, Copeland-Halperin LR, Suh VY, Yemc L. SPY Elite's Ability to Predict Nipple Necrosis in Nipple-Sparing Mastectomy and Immediate Tissue Expander Reconstruction. Plast Reconstr Surg - Glob Open. 2017;5(5):e1334. doi:10.1097/GOX.0000000000001334

48. Frey JD, Choi M, Karp NS. The Effect of Neoadjuvant Chemotherapy Compared to Adjuvant Chemotherapy in Healing after Nipple-Sparing Mastectomy. Plast Reconstr Surg. 2017;139(1):10e-19e. doi:10.1097/PRS.0000000000002841 
49. Hashem T, Farahat A. Thoracodorsal artery perforator flap as an autologous alternative to acellular dermal matrix. World J Surg Oncol. 2017;15(1):185. doi:10.1186/s12957-017-1254-9

50. Marquez MDLP, Fernandez-Riera R, Cardona HV, Flores JMR. Immediate implant replacement with DIEP flap: a single-stage salvage option in failed implant-based breast reconstruction. World J Surg Oncol. 2018;16(1):80. https://pubmed.ncbi.nlm.nih.gov/29665804.

51. Frey JD, Salibian AA, Levine JP, Karp NS, Choi M. Incision Choices in Nipple-Sparing Mastectomy: A Comparative Analysis of Outcomes and Evolution of a Clinical Algorithm. Plast Reconstr Surg. 2018;142(6):826e-835e. https://pubmed.ncbi.nlm.nih.gov/30204677.

52. Frey JD, Salibian AA, Karp NS, Choi M. The Impact of Mastectomy Weight on Reconstructive Trends and Outcomes in Nipple-Sparing Mastectomy: Progressively Greater Complications with Larger Breast Size. Plast Reconstr Surg. 2018;141(6):795e-804e. https://pubmed.ncbi.nlm.nih.gov/29794693.

53. Odom EB, Parikh RP, Um G, et al. Nipple-Sparing Mastectomy Incisions for Cancer Extirpation Prospective Cohort Trial: Perfusion, Complications, and Patient Outcomes. Plast Reconstr Surg. 2018;142(1):13-26. https://pubmed.ncbi.nlm.nih.gov/29878989.

54. Qureshi AA, Oliver JD, Parikh RP, Tenenbaum MM, Myckatyn TM. Salvage of Implant-Based Breast Reconstruction in Nipple-Sparing Mastectomies With Autologous Flaps. Aesthetic Surg J. 2018;38(7):734-741. https://pubmed.ncbi.nlm.nih.gov/29360922.

55. Roh TS, Kim JY, Jung BK, Jeong J, Ahn SG, Kim YS. Comparison of Outcomes between Direct-toImplant Breast Reconstruction Following Nipple-Sparing Mastectomy through Inframammary Fold Incision versus Noninframammary Fold Incision. J Breast Cancer. 2018;21(2):213-221. https://pubmed. ncbi.nlm.nih.gov/29963118.

56. Sarfati B, Struk S, Leymarie N, et al. Robotic Prophylactic Nipple-Sparing Mastectomy with Immediate Prosthetic Breast Reconstruction: A Prospective Study. Ann Surg Oncol. 2018;25(9):2579-2586. doi:10.1245/s10434-018-6555-x [doi]

57. de Vita R, Buccheri EM, Villanucci A, Pozzi M. Breast Reconstruction Actualized in Nipple-sparing Mastectomy and Direct-to-implant, Prepectoral Polyurethane Positioning: Early Experience and Preliminary Results. Clin Breast Cancer. 2019;19(2):e358-e363. https://pubmed.ncbi.nlm.nih. gov/30691930.

58. Ito H, Ueno T, Suga H, et al. Risk Factors for Skin Flap Necrosis in Breast Cancer Patients Treated with Mastectomy Followed by Immediate Breast Reconstruction. World J Surg. 2019;43(3):846-852. https:// pubmed.ncbi.nlm.nih.gov/30426185.

59. Lai H-W, Wang C-C, Lai Y-C, et al. The learning curve of robotic nipple sparing mastectomy for breast cancer: An analysis of consecutive 39 procedures with cumulative sum plot. Eur J Surg Oncol. 2019;45(2):125-133. https://pubmed.ncbi.nlm.nih.gov/30360987.

60. Ng T, Knowles S, Brackstone M, Doherty C. Mastectomy flap necrosis after nipple-sparing mastectomy and immediate implant-based reconstruction: An evaluation of tumescence and sharp dissection technique on surgical outcomes. Breast J. 2019;25(6):1079-1083. https://pubmed.ncbi.nlm.nih.gov/31359567.

61. Pallara T, Cagli B, Fortunato L, et al. Direct-To-Implant and 2-Stage Breast Reconstruction After Nipple Sparing Mastectomy: Results of a Retrospective Comparison. Ann Plast Surg. 2019:10.1097/ SAP.0000000000001893. https://pubmed.ncbi.nlm.nih.gov/31246667.

62. E.M. Q, M. BB, M. K, et al. Successful Immediate Staged Breast Reconstruction with Intermediary Autologous Lipotransfer in Irradiated Patients. Breast Care (Basel). 2019;145(2):S1072-7515(20)301162. https://pubmed.ncbi.nlm.nih.gov/31491673.

63. Reitsamer R, Peintinger F, Klaassen-Federspiel F, Sir A. Prepectoral direct-to-implant breast reconstruction with complete ADM or synthetic mesh coverage - 36-Months follow-up in 200 reconstructed breasts. Breast. 2019;48:32-37. https://pubmed.ncbi.nlm.nih.gov/31491673. 
64. Salibian AA, Frey JD, Bekisz JM, Karp NS, Choi M. Ischemic Complications after Nipple-sparing Mastectomy: Predictors of Reconstructive Failure in Implant-based Reconstruction and Implications for Decision-making. Plast Reconstr surgery Glob open. 2019;7(5):e2280-e2280. https://pubmed.ncbi.nlm. nih.gov/31333984.

65. Tasoulis M-K, Agusti A, Karakatsanis A, Montgomery C, Marshall C, Gui G. The Use of Hydrodissection in Nipple- and Skin-sparing Mastectomy. Plast Reconstr Surg - Glob Open. 2019;7(11):e2495. doi:10.1097/GOX.0000000000002495

66. Yang C-Q, Ji F, Gao H-F, et al. The Role of Sharp Dissection in Nipple-Sparing Mastectomy: A Safe Procedure with No Necrosis of the Nipple-Areolar Complex. Cancer Manag Res. 2019;11:10223-10228. https://pubmed.ncbi.nlm.nih.gov/31824192.

67. Young WA, Degnim AC, Hoskin TL, et al. Outcomes of $>1300$ Nipple-Sparing Mastectomies with Immediate Reconstruction: The Impact of Expanding Indications on Complications. Ann Surg Oncol. 2019;26(10):3115-3123. https://pubmed.ncbi.nlm.nih.gov/31342370.

68. Chan YH-Y, Yue IK-H, Ho C-M, Cheung PS-Y. The Use of Serratus Anterior Fascial Flap in Integrated Mastectomy and Implant Reconstruction. World J Surg. 2020;44(3):825-830. https://pubmed.ncbi.nlm. nih.gov/31732761.

69. Lai H-W, Chen S-T, Tai C-M, et al. Robotic- Versus Endoscopic-Assisted Nipple-Sparing Mastectomy with Immediate Prosthesis Breast Reconstruction in the Management of Breast Cancer: A Case-Control Comparison Study with Analysis of Clinical Outcomes, Learning Curve, Patient-Reported Aestheti. Ann Surg Oncol. 2020:10.1245/s10434-020-08223-0. https://pubmed.ncbi.nlm.nih.gov/32016631.

70. Moon K-C, Baek S-O, Yoon E-S, Lee B-I, Park S-H. Predictors affecting complications and aesthetic outcomes in autologous breast reconstruction with free muscle-sparing transverse rectus abdominis myocutaneous flaps. Microsurgery. 2020;40(1):38-43. https://pubmed.ncbi.nlm.nih.gov/30793787.

71. Park KU, Weiss A, Rosso K, et al. Use of Mammographic Measurements to Predict Complications After Nipple-Sparing Mastectomy in BRCA Mutation Carriers. Ann Surg Oncol. 2020;27(2):367-372. https:// pubmed.ncbi.nlm.nih.gov/31399819.

72. Salibian AA, Bekisz JM, Frey JD, Miller B, Choi M, Karp NS. Prophylactic nipple-sparing mastectomy in young previvors: Examining decision-making, reconstructive outcomes, and patient satisfaction in BRCA+ patients under 30. Breast J. 2020;26(5):971-975. doi:10.1111/tbj.13692

73. Seki H, Sakurai T, Maeda Y, et al. Utility of the periareolar incision technique for breast reconstructive surgery in patients with breast cancer. Surg Today. 2020:10.1007/s00595-020-01975-y. https://pubmed. ncbi.nlm.nih.gov/32052184.

74. Valero MG, Muhsen S, Moo T-A, et al. Increase in Utilization of Nipple-Sparing Mastectomy for Breast Cancer: Indications, Complications, and Oncologic Outcomes. Ann Surg Oncol. 2020;27(2):344-351. https://pubmed.ncbi.nlm.nih.gov/31823173.

75. Wang M, Huang J, Chagpar AB. Is nipple sparing mastectomy associated with increased complications, readmission and length of stay compared to skin sparing mastectomy? Am J Surg. 2020;219(6):10301035. doi:10.1016/j.amjsurg.2019.09.011

76. Willey SC, Fan KL, Luvisa K, et al. Predicting Ischemic Complications in the Inframammary Approach to Nipple-Sparing Mastectomy: The Midclavicular-to-Inframammary Fold Measurement. Plast Reconstr Surg. 2020;145(2):251e-262e. https://pubmed.ncbi.nlm.nih.gov/31985611. 


\section{Appendix 3: studies included in the analysis}

Table 1. Baseline characteristics of studies on nipple sparing mastectomy

\begin{tabular}{|c|c|c|c|c|c|c|c|c|c|}
\hline Author & year & country & type & NSM & $\mathrm{C1}$ & $\mathrm{C} 2$ & C3 & $\mathrm{C4}$ & $\mathrm{C} 5$ \\
\hline Frey et al. ${ }^{38}$ & 2017 & USA & NSM & 1028 & A & $\mathrm{B}$ & $\mathrm{C}$ & $\mathrm{D}$ & $\mathrm{E}$ \\
\hline Rancati et al. ${ }^{39}$ & 2017 & Argentina & NSM & 30 & B & $\mathrm{C}$ & $\mathrm{D}$ & & \\
\hline Frey et al. ${ }^{40}$ & 2017 & USA & NSM & 543 & A & B & $\mathrm{C}$ & $\mathrm{D}$ & $\mathrm{E}$ \\
\hline Frey et al. ${ }^{41}$ & 2017 & USA & NSM & 420 & $\mathrm{D}$ & & & & \\
\hline Mesdag et al. ${ }^{11}$ & 2017 & France & NSM & 63 & A & $\mathrm{C}$ & $\mathrm{D}$ & $\mathrm{E}$ & \\
\hline Dull et al. ${ }^{42}$ & 2017 & USA & NSM & 197 & A & B & C & $\mathrm{D}$ & $\mathrm{E}$ \\
\hline Lago et al. ${ }^{12}$ & 2017 & Spain & NSM & 69 & $\mathrm{D}$ & & & & \\
\hline Murphy et al. ${ }^{43}$ & 2017 & USA & NSM & 19 & B & $\mathrm{D}$ & & & \\
\hline Chan et al. ${ }^{13}$ & 2017 & Hong Kong & NSM & 103 & $\mathrm{C}$ & E & & & \\
\hline Colwell et al. ${ }^{44}$ & 2017 & USA & NSM & 32 & A & B & $\mathrm{C}$ & $\mathrm{D}$ & $\mathrm{E}$ \\
\hline De Vita et al. ${ }^{45}$ & 2017 & Italy & NSM & 2023 & A & B & $\mathrm{C}$ & $\mathrm{D}$ & $\mathrm{E}$ \\
\hline Sbitany et al. ${ }^{46}$ & 2017 & USA & NSM & 270 & A & B & $\mathrm{C}$ & $\mathrm{D}$ & $\mathrm{E}$ \\
\hline Toesca et al. ${ }^{16}$ & 2017 & Italy & RNSM & 29 & A & B & $\mathrm{C}$ & $\mathrm{D}$ & $\mathrm{E}$ \\
\hline Venturi et al. ${ }^{47}$ & 2017 & USA & NSM & 32 & $\mathrm{D}$ & $\mathrm{E}$ & & & \\
\hline Frey et al. ${ }^{48}$ & 2017 & USA & NSM & 798 & A & B & $\mathrm{C}$ & $\mathrm{D}$ & $\mathrm{E}$ \\
\hline Hashem et al. ${ }^{49}$ & 2017 & Egypt & NSM & 55 & A & & & & \\
\hline Casella et al. ${ }^{7}$ & 2018 & Italy & NSM & 92 & A & B & $\mathrm{C}$ & $\mathrm{D}$ & $\mathrm{E}$ \\
\hline de la Parra Marquez et al. ${ }^{50}$ & 2018 & Mexico & NSM & 140 & B & $\mathrm{C}$ & $\mathrm{D}$ & $\mathrm{E}$ & \\
\hline Frey et al. ${ }^{51}$ & 2018 & USA & NSM & 1207 & A & B & $\mathrm{C}$ & $\mathrm{D}$ & $\mathrm{E}$ \\
\hline Frey et al. ${ }^{52}$ & 2018 & USA & NSM & 809 & A & B & $\mathrm{C}$ & $\mathrm{D}$ & $\mathrm{E}$ \\
\hline Lai et al. ${ }^{18}$ & 2018 & Taiwan & RNSM & 15 & A & B & $\mathrm{C}$ & $\mathrm{D}$ & $\mathrm{E}$ \\
\hline Odom et al. ${ }^{53}$ & 2018 & USA & NSM & 79 & A & B & $\mathrm{C}$ & $\mathrm{D}$ & $\mathrm{E}$ \\
\hline Pek et al. ${ }^{9}$ & 2018 & Singapore & NSM & 142 & A & C & & & \\
\hline Qureshi et al. ${ }^{54}$ & 2018 & USA & NSM & 382 & A & B & $\mathrm{C}$ & $\mathrm{E}$ & \\
\hline Roh et al. ${ }^{55}$ & 2018 & South Korea & NSM & 145 & A & B & $\mathrm{C}$ & $\mathrm{D}$ & $\mathrm{E}$ \\
\hline Sarfati et al. ${ }^{56}$ & 2018 & France & RNSM & 63 & A & B & $\mathrm{C}$ & $\mathrm{E}$ & \\
\hline de Vita et al. ${ }^{57}$ & 2019 & Italy & NSM & 34 & A & B & $\mathrm{C}$ & $\mathrm{D}$ & \\
\hline Houvenaeghel et al. ${ }^{19}$ & 2019 & France & RNSM & 27 & A & $\mathrm{C}$ & $\mathrm{E}$ & & \\
\hline Ito et al. 58 & 2019 & Japan & NSM & 123 & $\mathrm{D}$ & & & & \\
\hline Kim et al. ${ }^{8}$ & 2019 & South Korea & NSM & 55 & A & B & $\mathrm{C}$ & $\mathrm{E}$ & \\
\hline Lai et al. ${ }^{59}$ & 2019 & Taiwan & RNSM & 39 & A & B & $\mathrm{D}$ & $\mathrm{E}$ & \\
\hline $\mathrm{Ng}$ et al. ${ }^{60}$ & 2019 & Canada & NSM & 116 & A & B & $\mathrm{D}$ & E & \\
\hline
\end{tabular}




\begin{tabular}{|c|c|c|c|c|c|c|c|c|c|}
\hline Author & year & country & type & NSM & $\mathrm{C1}$ & $\mathrm{C} 2$ & C3 & $\mathrm{C4}$ & $\mathrm{C5}$ \\
\hline Pallara et al. ${ }^{61}$ & 2019 & Italy & NSM & 106 & $\mathrm{~A}$ & $\mathrm{~B}$ & $\mathrm{C}$ & $\mathrm{D}$ & $\mathrm{E}$ \\
\hline Park et al. ${ }^{17}$ & 2019 & South Korea & RNSM & 12 & A & B & $\mathrm{C}$ & $\mathrm{E}$ & \\
\hline Quinn et al. ${ }^{62}$ & 2019 & Ireland & NSM & 43 & A & C & $\mathrm{E}$ & & \\
\hline Reitsamer et al. ${ }^{63}$ & 2019 & Austria & NSM & 200 & A & B & $\mathrm{E}$ & & \\
\hline Salibian et al. ${ }^{64}$ & 2019 & USA & NSM & 1045 & A & B & C & $\mathrm{D}$ & $\mathrm{E}$ \\
\hline Tasoulis et al. ${ }^{65}$ & 2019 & United Kingdom & NSM & 82 & C & $\mathrm{D}$ & $\mathrm{E}$ & & \\
\hline Yang et al. ${ }^{66}$ & 2019 & China & NSM & 130 & C & $\mathrm{D}$ & & & \\
\hline Young et al. ${ }^{67}$ & 2019 & USA & NSM & 1301 & A & B & $\mathrm{C}$ & $\mathrm{D}$ & \\
\hline Chan et al. ${ }^{68}$ & 2020 & Hong Kong & NSM & 61 & $\mathrm{E}$ & & & & \\
\hline Lai et al. ${ }^{69}$ & 2020 & Taiwan & RNSM & 40 & A & B & $\mathrm{D}$ & $\mathrm{E}$ & \\
\hline Moon et al. ${ }^{70}$ & 2020 & South Korea & NSM & 214 & A & B & $\mathrm{D}$ & & \\
\hline Park et al. ${ }^{71}$ & 2020 & USA & NSM & 114 & A & B & $\mathrm{C}$ & $\mathrm{D}$ & $\mathrm{E}$ \\
\hline Salibian et al. ${ }^{72}$ & 2020 & USA & NSM & 44 & A & C & $\mathrm{D}$ & & \\
\hline Seki et al. ${ }^{73}$ & 2020 & Japan & NSM & 181 & A & $\mathrm{C}$ & $\mathrm{D}$ & $\mathrm{E}$ & \\
\hline Valero et al. ${ }^{74}$ & 2020 & USA & NSM & 777 & A & $\mathrm{C}$ & $\mathrm{E}$ & & \\
\hline Wang et al. ${ }^{75}$ & 2020 & USA & NSM & 217 & B & $\mathrm{C}$ & $\mathrm{D}$ & & \\
\hline Willey et al. ${ }^{76}$ & 2020 & USA & NSM & 140 & A & B & C & E & \\
\hline
\end{tabular}

$\mathrm{C} \#$ = Complication, $\mathrm{A}=$ hematoma, $\mathrm{B}=$ seroma, $\mathrm{C}=$ infection, $\mathrm{D}=$ flap necrosis, $\mathrm{E}=$ implant removal, NSM = nipple sparing mastectomy, RNSM = robot assisted nipple sparing mastectomy, USA = United States of America. 


\section{Appendix 4: Risk of bias assessment}

Supplementary table2. Risk of bias for different studies.

\begin{tabular}{|c|c|c|c|c|c|c|c|}
\hline Author & D1 & D2 & D3 & D4 & D5 & D6 & D7 \\
\hline Houvenaeghel & Moderate & Low & Low & Moderate & Low & Low & Low \\
\hline de Vita & Low & Low & Low & Low & Low & Low & Low \\
\hline Kim & Low & Low & Low & Low & Moderate & Low & Low \\
\hline Roh & Low & Low & Low & Low & Low & Low & Low \\
\hline Pallara & Moderate & Low & Low & Low & Low & Low & Low \\
\hline Pek & Low & Low & Low & Low & Low & Low & Low \\
\hline Quinn & Low & Low & Low & Low & Low & Low & Low \\
\hline de la Parra Marquez & Low & Low & Low & Low & Low & Low & Low \\
\hline Frey & Low & Low & Low & Low & Low & Low & Low \\
\hline Valero & Low & Low & Low & Low & Low & Low & Low \\
\hline Wang & Low & Low & Low & Low & Low & Low & Low \\
\hline Salibian & Low & Low & Low & Low & Low & Low & Moderate \\
\hline $\mathrm{Ng}$ & Low & Low & Low & Low & Low & Low & Low \\
\hline Casella & Low & Low & Low & Low & Low & Low & Low \\
\hline Odom & Moderate & Low & Low & Low & Low & Moderate & Low \\
\hline Young & Moderate & Low & Low & Moderate & Low & Low & Low \\
\hline Willey & Low & Low & Low & Low & Low & Low & Low \\
\hline Moon & Moderate & Low & Moderate & Low & Low & Low & Low \\
\hline Reitsamer & Low & Low & Low & Low & Low & Low & Low \\
\hline Salibian & Moderate & Moderate & Low & Moderate & Low & Low & Moderate \\
\hline Ito & Low & Low & Low & Low & Low & Low & Low \\
\hline Lai & Low & Low & Low & Low & Low & Low & Low \\
\hline Park & Low & Low & Low & Low & Low & Low & Low \\
\hline Sarfati & Low & Low & Low & Low & Low & Low & Low \\
\hline Lai & Low & Low & Low & Low & Low & Low & Low \\
\hline Qureshi & Low & Low & Low & Low & Moderate & Low & Low \\
\hline Frey & Low & Low & Low & Low & Low & Low & Low \\
\hline Lai & Low & Low & Low & Low & Low & Low & Low \\
\hline Yang & Low & Low & Low & Low & Low & Low & Low \\
\hline Tasoulis & Low & Low & Low & Low & Low & Low & Low \\
\hline Chan & Low & Low & Low & Low & Low & Low & Low \\
\hline Park & Low & Low & Low & Low & Low & Low & Low \\
\hline Seki & Low & Low & Low & Low & Low & Low & Low \\
\hline
\end{tabular}




\begin{tabular}{llllllll}
\hline Author & D1 & D2 & D3 & D4 & D5 & D6 & D7 \\
\hline Frey & Low & Low & Low & Low & Low & Low & Low \\
Rancati & Low & Low & Low & Low & Low & Low & Low \\
Frey & Low & Low & Low & Low & Low & Low & Low \\
Frey & Low & Low & Low & Low & Low & Low & Low \\
Mesdag & Low & Low & Low & Low & Low & Low & Low \\
Dull & Low & Low & Low & Low & Low & Low & Low \\
Lago & Low & Low & Low & Low & Low & Low & Low \\
Murphy & Low & Low & Low & Low & Low & Low & Low \\
Chan & Low & Low & Low & Low & Low & Low & Low \\
Colwell & Low & Low & Low & Low & Low & Low & Low \\
De Vita & Low & Low & Low & Low & Low & Low & Low \\
Sbitany & Low & Low & Low & Low & Low & Low & Low \\
Toesca & Low & Low & Low & Low & Low & Low & Low \\
Venturi & Low & Low & Low & Low & Low & Low & Low \\
Frey & Moderate & Low & Low & Low & Low & Low & Low \\
Hashem & Low & Low & Low & Low & Low & Low \\
\hline & Low & & & & & & Low
\end{tabular}

$\mathrm{D} 1=$ Bias due to confounding D2 = Bias in selection of participants into the study; D3 = Bias in classification of interventions; D4 = Bias due to deviations from intended interventions; D5 = Bias due to missing data; D6 $=$ Bias in measurements of outcomes; D7 = Bias in selection of the reported result. 


\section{Appendix 5: supplementary figures}

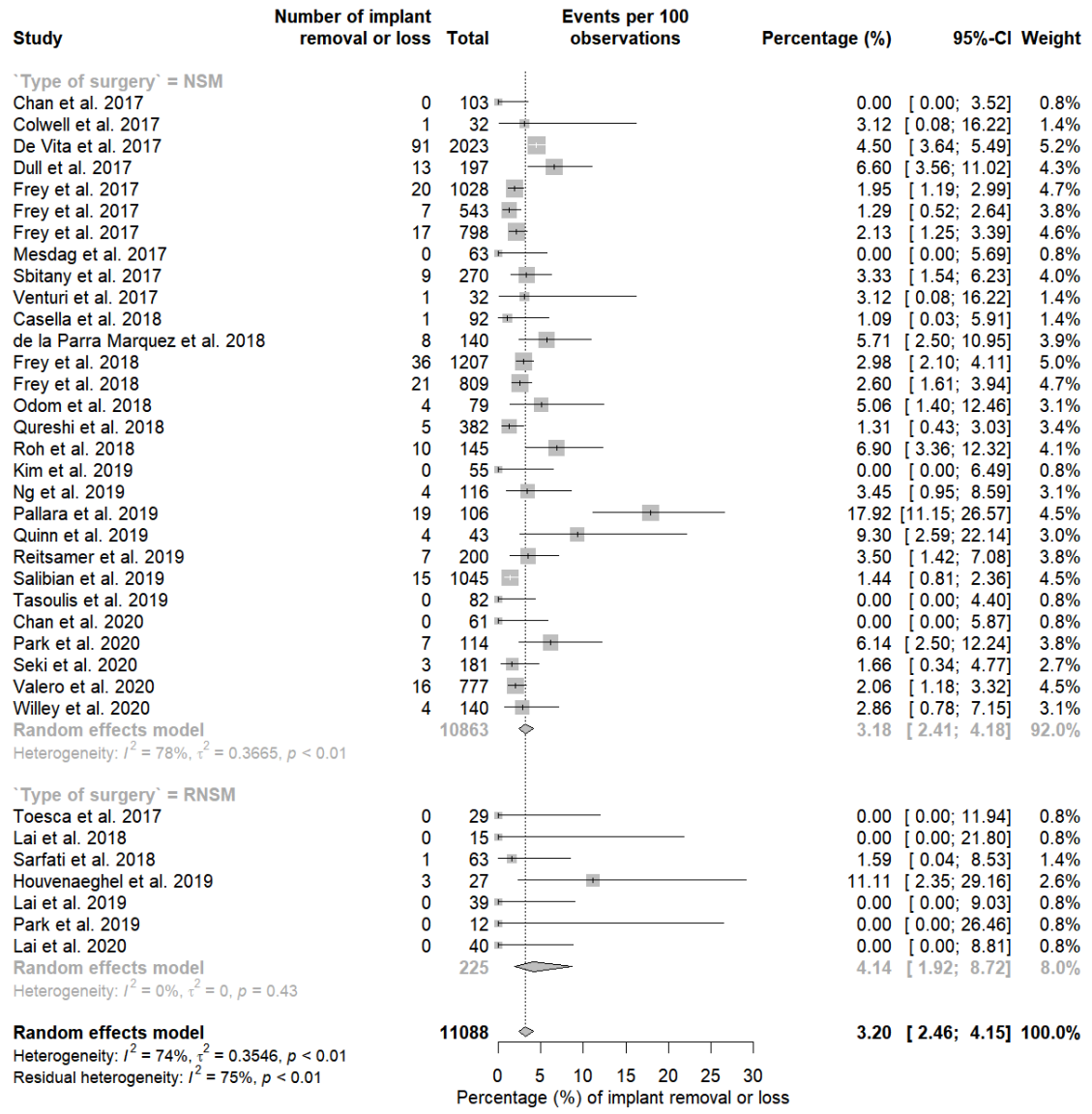

Supplementary Figure 1. Postoperative Implant loss. $\mathrm{P}=0.523,(\mathrm{R}) \mathrm{NSM}=$ (Robotic) nipple sparing mastectomy, $\mathrm{CI}=$ confidence interval. 


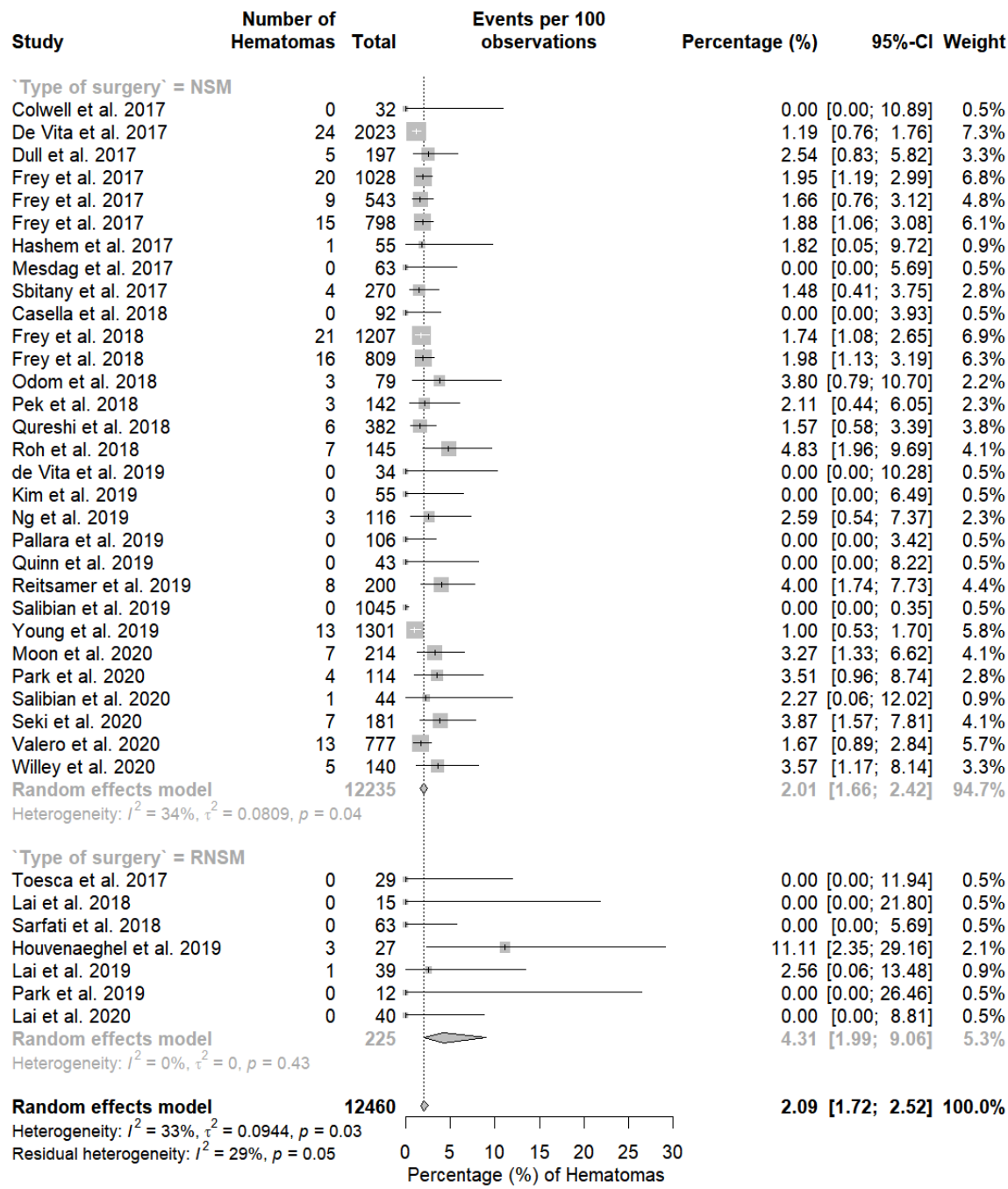

Supplementary Figure 2. Hematomas. $\mathrm{P}=0.059$, (R)NSM $=$ (Robotic) nipple sparing mastectomy, $\mathrm{CI}=$ confidence interval. 
Robotic nipple sparing mastectomy complication rate compared to traditional nipple sparing mastectomy:

a systematic review and meta-analysis

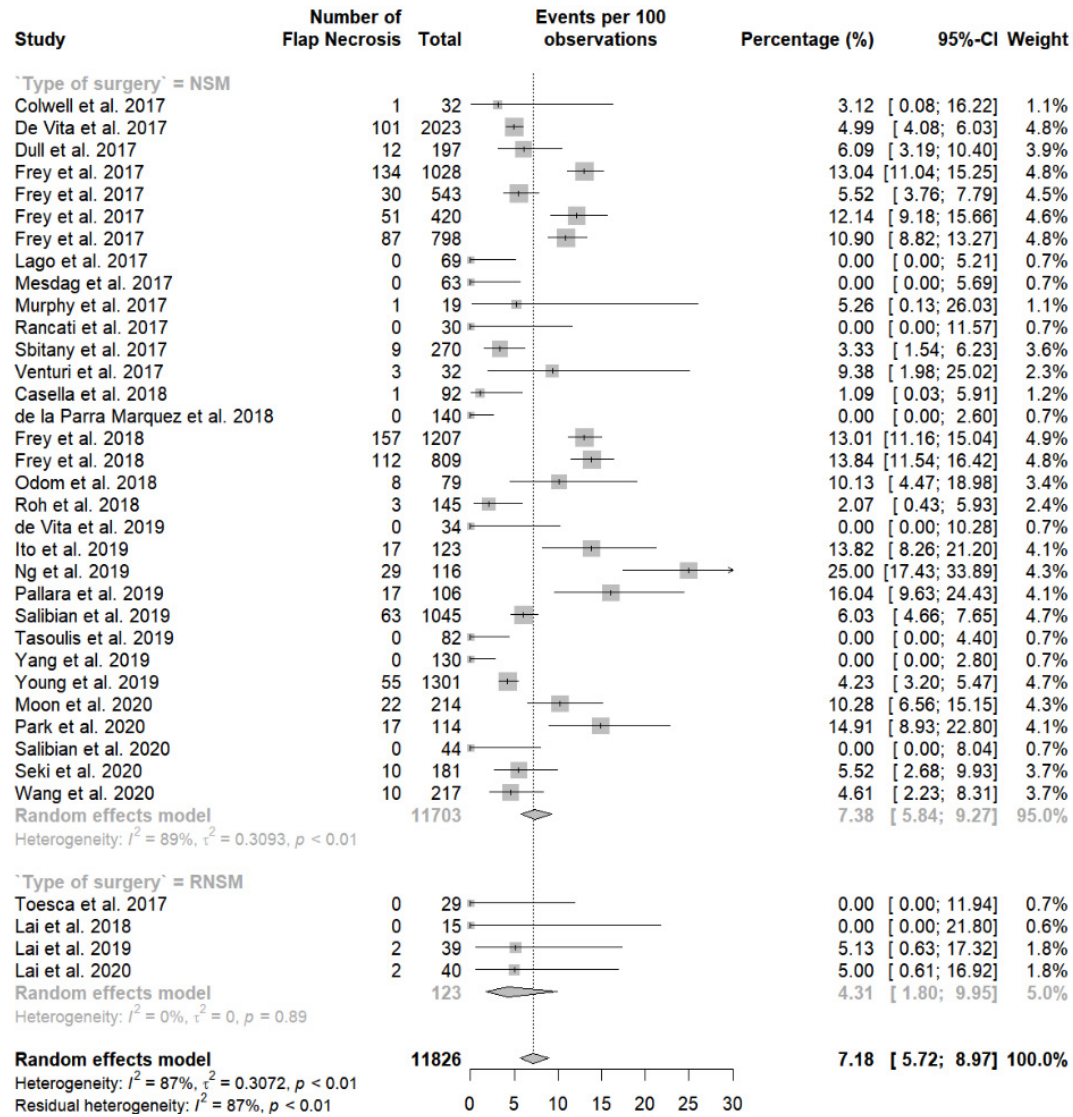

Supplementary Figure 3. Flap necrosis. $\mathrm{P}=0.230,(\mathrm{R}) \mathrm{NSM}=$ (Robotic) nipple sparing mastectomy, $\mathrm{CI}=$ confidence interval. 


\section{Study}

'Type of surgery' = NSM

Chan et al. 2017

Colwell et al. 2017

De Vita et al. 2017

Dull et al. 2017

Frey et al. 2017

Frey et al. 2017

Frey et al. 2017

Mesdag et al. 2017

Rancati et al. 2017

Sbitany et al. 2017

Casella et al. 2018

de la Parra Marquez et al. 2018

Frey et al. 2018

Frey et al. 2018

Odom et al. 2018

Pek et al. 2018

Qureshi et al. 2018

Roh et al. 2018

de Vita et al. 2019

Kim et al. 2019

Pallara et al. 2019

Quinn et al. 2019

Salibian et al. 2019

Tasoulis et al. 2019

Yang et al. 2019

Young et al. 2019

Park et al. 2020

Salibian et al. 2020

Seki et al. 2020

Valero et al. 2020

Wang et al. 2020

Willey et al. 2020

Random effects model

Heterogeneity: $I^{2}=85 \%, \tau^{2}=0.4729, p<0.01$

'Type of surgery' = RNSM

Toesca et al. 2017

Lai et al. 2018

Sarfati et al. 2018

Houvenaeghel et al. 2019

Park et al. 2019

Random effects model

Heterogeneity: $I^{2}=14 \%, \tau^{2}=0.1006, p=0.33$

Random effects model

Heterogeneity: $I^{2}=83 \%, \tau^{2}=0.4679, p<0.01$

Residual heterogeneity: $I^{2}=83 \%, p<0.01$ infections Total observations
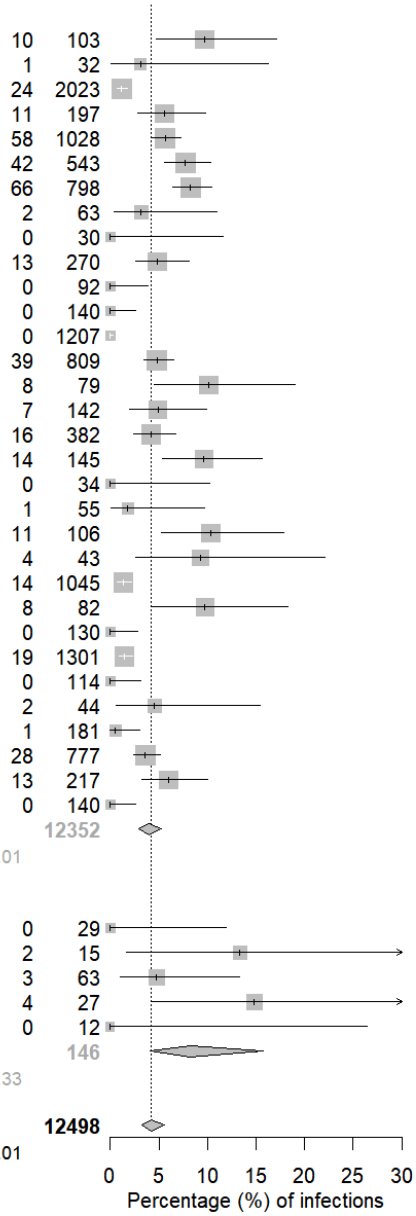

Percentage (\%) 95\%-Cl Weight

$9.71[4.75 ; 17.13] \quad 3.7 \%$

$3.12[0.08 ; 16.22] \quad 1.4 \%$

$1.19[0.76 ; 1.76] \quad 4.2 \%$

$5.58 \quad[2.82 ; 9.77] \quad 3.8 \%$

$5.64 \quad[4.31 ; 7.23] \quad 4.4 \%$

$7.73[5.63 ; 10.31] \quad 4.4 \%$

$8.27[6.45 ; 10.40] \quad 4.5 \%$

$3.17[0.39 ; 11.00] \quad 2.2 \%$

$0.00[0.00 ; 11.57] \quad 0.9 \%$

$4.81 \quad[2.59 ; 8.09] \quad 3.9 \%$

$0.00 \quad[0.00 ; 3.93] \quad 0.9 \%$

$0.00[0.00 ; 2.60] \quad 0.9 \%$

$0.00 \quad[0.00 ; 0.31] \quad 0.9 \%$

$4.82[3.45 ; 6.53] \quad 4.4 \%$

$10.13[4.47 ; 18.98] \quad 3.6 \%$

$4.93 \quad[2.00 ; 9.89] \quad 3.5 \%$

$4.19 \quad[2.41 ; 6.71] \quad 4.1 \%$

$9.66[5.38 ; 15.67] \quad 3.9 \%$

$0.00[0.00 ; 10.28] \quad 0.9 \%$

$1.82[0.05 ; 9.72] \quad 1.5 \%$

$10.38[5.30 ; 17.81] \quad 3.8 \%$

$9.30[2.59 ; 22.14] \quad 2.9 \%$

$1.34[0.73 ; 2.24] \quad 4.0 \%$

$9.76[4.31 ; 18.32] \quad 3.6 \%$

$0.00 \quad[0.00 ; 2.80] \quad 0.9 \%$

$1.46 \quad[0.88 ; 2.27] \quad 4.1 \%$

$0.00 \quad[0.00 ; 3.18] \quad 0.9 \%$

$4.55[0.56 ; 15.47] \quad 2.2 \%$

$0.55[0.01 ; 3.04] \quad 1.5 \%$

$3.60[2.41 ; 5.17] \quad 4.3 \%$

$5.99[3.23 ; 10.03] \quad 3.9 \%$

$0.00[0.00 ; 2.60] \quad 0.9 \%$

$3.96[2.96 ; 5.30] \quad 90.7 \%$

$0.00[0.00 ; 11.94] \quad 0.9 \%$

$13.33[1.66 ; 40.46] \quad 2.1 \%$

$4.76[0.99 ; 13.29] \quad 2.6 \%$

$14.81[4.19 ; 33.73] \quad 2.8 \%$

$0.00[0.00 ; 26.46] \quad 0.8 \%$

$8.26[4.15 ; 15.78] \quad 9.3 \%$

$4.22[3.20 ; 5.56] 100.0 \%$

Supplementary Figure 4. Infections. $\mathrm{P}=0.054,(\mathrm{R}) \mathrm{NSM}=$ (Robotic) nipple sparing mastectomy, $\mathrm{CI}=$ confidence interval. 
Robotic nipple sparing mastectomy complication rate compared to traditional nipple sparing mastectomy:

a systematic review and meta-analysis

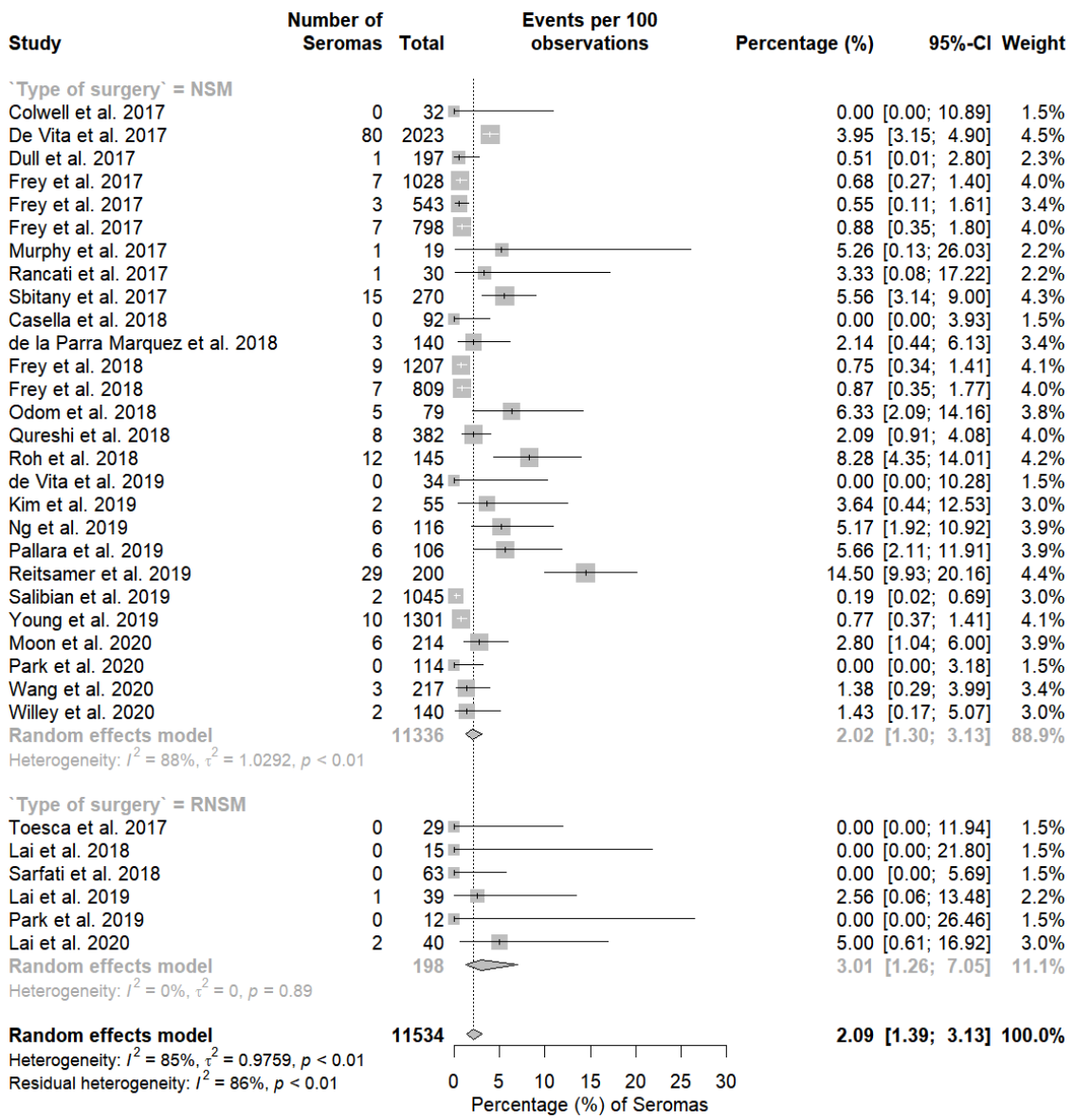

Supplementary Figure 5. Seroma. $\mathrm{P}=0.421$, (R)NSM = (Robotic) nipple sparing mastectomy, $\mathrm{CI}=$ confidence interval. 


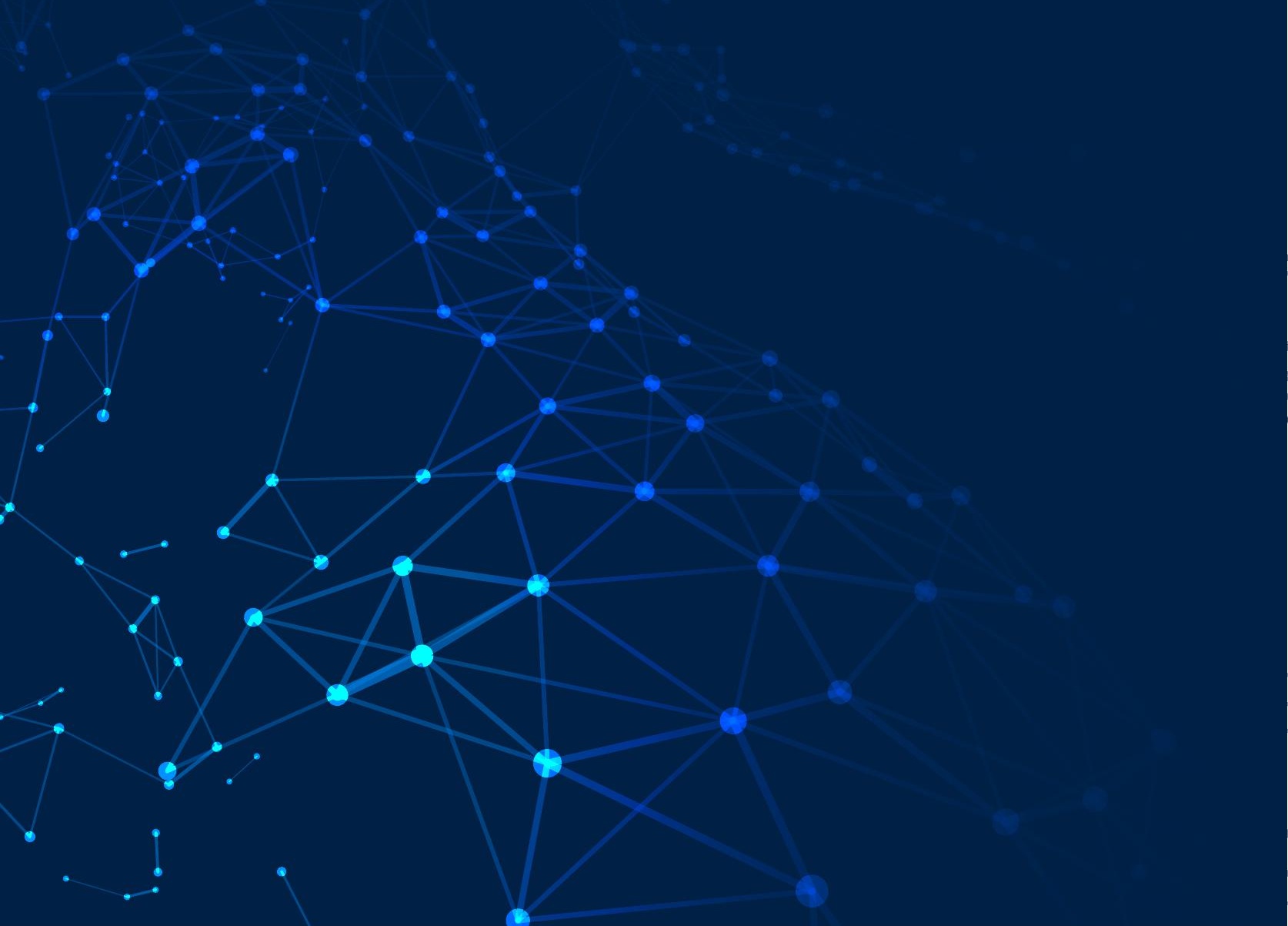





\section{Chapter 10}

The association of socioeconomic status on treatment strategy in patients with stage I and II breast cancer in the Netherlands

M.D. Filipe

S. Siesling

M.R. Vriens

P. van Diest

A.J. Witkamp 


\section{Abstract}

\section{Background}

Previous studies have shown that socioeconomic status (SES) influences breast cancer therapy. However, these studies were performed in countries with unequal access to healthcare. Therefore, the aim of this study is to investigate whether SES also contributes to the likelihood of receiving a certain therapy in The Netherlands, a country with supposedly equal access to healthcare.

\section{Materials and methods}

From the Netherlands Cancer Registry, 105,287 patients with newly diagnosed stage I or II breast cancer diagnosed between 2011 and 2018 were selected for analysis. SES was calculated from the average incomes of each postal code, which were divided into 10-deciles. Primary outcome was the effect of SES on the likelihood of undergoing surgery and secondary outcome was the effect of SES on the likelihood of the type of surgery. Both outcomes were corrected for patient, tumor and hospital characteristics and were expressed as odds ratio (OR) with 95\% confidence interval (CI).

\section{Results}

SES did not affect the likelihood of a breast cancer patient to undergo surgery (OR 1.00 per $10 \%$ stratum). In contrast, increased age and higher tumor stage were the most important factors determining whether patients underwent surgery.

Patients with higher SES were less likely to undergo mastectomy (OR 0.98). Additionally, more recently diagnosed patients were less likely to undergo mastectomy (OR 0.93 per year) while patients with higher tumor stage were more likely to undergo mastectomy (OR 3.42).

\section{Conclusion:}

SES does not affect whether a patient undergoes surgery or not but does affect the type of surgery. 


\section{Introduction}

Breast cancer is the most common cancer in women and the second most common cause of death due to cancer in women worldwide. ${ }^{1}$ There are roughly 17.000 new cases of breast cancer in The Netherlands every year. Additionally, over 3.000 people of the Dutch population die annually due to breast cancer. ${ }^{2,3}$

Surgical resection of the primary tumor is the treatment of choice in patients with newly diagnosed breast cancer. Tumor stage and molecular characteristics determine the type of surgery. The main types of surgery for stage I and II are mastectomy and breast conserving therapy (BCT). ${ }^{4-6}$ In The Netherlands, the percentage BCT is about $65 \%$ and this has been shown to differ between regions ${ }^{7}$. Reasons for these differences can be the preference of the clinician, age of patient, tumor grade, tumor stage and hormone receptor status. ${ }^{7}$ Moreover, socioeconomic status (SES) might be of influence here since it is not equally spread over the country. ${ }^{8}$

SES is a complex classification system to stratify economic and social factors. ${ }^{9}$ SES has shown to be of influence the incidence and severity of diseases. Low SES is associated with a higher incidence of lifestyle related risk factors such as smoking, higher BMI and drug use. ${ }^{10}$ This high risk behavior leads to an increase risk for the development of disease such as diabetes, cardiovascular disease, psychiatric disorders and numerous types of cancer. ${ }^{10-14}$

Differences in treatment between SES classes have been described in a systematic review in which patients with breast cancer who, among other factors, had a higher SES were more likely to undergo BCT. ${ }^{15}$ Additionally, in the United States of America unequal access to healthcare due to financial barriers leads to therapeutic choices based on income. ${ }^{16}$ Various studies in the United States showed that SES influences the choice for the surgical procedure ${ }^{17,18}$. Furthermore, a Danish study showed that low SES stage I or II breast cancer patients tended to have more mastectomies despite equal access to healthcare. There was no clear explanation for this disparity. ${ }^{19}$ However, this was not a population-based cohort, and the study cohort was closed 1998 while treatment options have changed since then.

In The Netherlands, there is universal healthcare which means that citizens do not have financial barriers when requesting medical attention. ${ }^{16}$ Additionally, the compulsory insurance covers almost all costs for hospitals and primary care. ${ }^{16,20}$

Currently no studies have analyzed whether SES influences treatment choices and type of surgery in patients with stage I and II breast cancer in a situation in which no financial barriers for access to care. The aim of this study was therefore to determine whether treatment choices are influenced SES in The Netherlands where there no financial care access barriers. 


\section{Materials and methods}

\section{Study design and population}

In this nationwide population-based study, we selected breast cancer patients of the Netherlands Cancer Registry (NCR). The present study focused on primary stage I and II breast cancer patients treated between January $1^{\text {st }}, 2011$ and December 31 $1^{\text {st }}, 2018$. Only new onset breast cancer patients were included in this study.

\section{Definitions}

The NCR contains patient-, tumor- and treatment characteristics. Tumors are categorized according to the tumor, node and metastasis (TNM) classification system ${ }^{21}$. Due to changes in the $\mathrm{N} 1$ category from the $5^{\text {th }}$ to the $6^{\text {th }}$ editions of the International Classification of Diseases for Oncology, we classified the number of positive lymph into $\mathrm{N}$ categories. Patients without lymph node involvement were classified as N0 and patients with 1 to 3 positive lymph nodes were classified as N1. TNM was converted to tumor stage (stage I or stage II). Histological subtype consisted of lobular, ductal, mucinous, medullary tubular or not specified. ${ }^{22}$ Tumor grade was divided into low, intermediate and high grade. ${ }^{23}$

SES was determined using the average income of a household according to the four-digit postal code in The Netherlands at time of diagnosis and surgical procedure, and was defined according to the Dutch Bureau of Statistics (CBS). ${ }^{24}$ Furthermore, the average incomes of each postal code were divided into 10-deciles. Additionally, hospital volume was stratified based on the number of breast cancer patients treated per year; low $(<100)$, medium (100-149) and high volume (>150), as described in previous studies. ${ }^{25}$

\section{Outcomes}

Primary outcome was the effect of SES on the likelihood of a new onset breast cancer patients undergoing surgery versus no surgical treatment. Secondary outcome was determining the effect of SES on type of surgery (BCT or mastectomy). Both outcomes were determined after correcting for other patient, tumor and hospital characteristics.

\section{Statistics}

Descriptive statistics were used to describe patient, tumor and treatment characteristics. Continuous data were described with mean along with standard deviation (SD), or with median and interquartile range (IQR), depending on whether or not the data were normally distributed. Mann-Whitney-U tests or Student's t-tests were used to test differences between groups of not normally and normally distributed continuous data, respectively. Differences between categorical data were analyzed with Chi-Square or Fisher's exact tests. 
Since some data was missing during the study period, multiple imputation by chained equations (MICE) were performed using the MICE package in R. After comparing and correlating the missing to the non-missing data, it was concluded that the values were missing at random. The imputation was repeated 20 times, followed by application of Rubin's rule to combine parameter estimates and standard errors. ${ }^{26,27}$ Imputed data was later compared to the complete cases to determine validity of the imputation model. Subsequently, the imputed data was used for analyses.

Multivariable regression analyses were performed to study the association between SES and the likelihood (quantified in odds ratio [OR] and $95 \%$ confidence interval $[\mathrm{CI}]$ ) of undergoing a certain treatment strategy (no surgery vs. surgery, and BCT vs. mastectomy) in patients with stage I or II breast cancer. Possible confounding factors and effect modifiers considered were age at diagnosis, stage ( 1 or 2 ) and co-morbidities. Twosided P-values below 0.05 were considered statistically significant.

All calculations were performed using RStudio 1.2.5001 (with R version: x64 3.6.3). Visualization of plots was performed using the ggplot2 package. 


\section{Results}

Between 2011 and 2018, 105,287 patients had new onset stage I or II breast cancer, of which 6,840 patients $(6.5 \%)$ did not undergo surgery. Furthermore, 98,447 stage I or II breast cancer patients underwent surgery of which 65,888 patients underwent BCT and 32,559 patients had undergone mastectomy suitable for analysis.

Table 1 shows the baseline characteristics of all 105,287 stage I or II breast cancer patients suitable for analysis. Mean age was 62.0 years. The national screening program detected breast cancer in 39,094 (37.7\%) patients and $393(0.4 \%)$ patients had a positive oncological history other than breast cancer. A total of $98,447(93.5 \%)$ stage I or II breast cancer patients underwent surgery (BCT or mastectomy) while 6,840 (6.5\%) breast cancer patients had no surgery but were treated with only chemotherapy, hormone therapy and/or radiotherapy. The proportion of breast cancer patients undergoing nonsurgical treatment slightly increased over time (Figure 1). Stage I or II breast cancer was evenly spread among the different strata of SES. The proportion of BCT substantially increases while the proportion mastectomies decreases. 
Table 1. Baseline characteristics of all new onset breast cancer patients diagnosed in The Netherlands between 2011 and 2018.

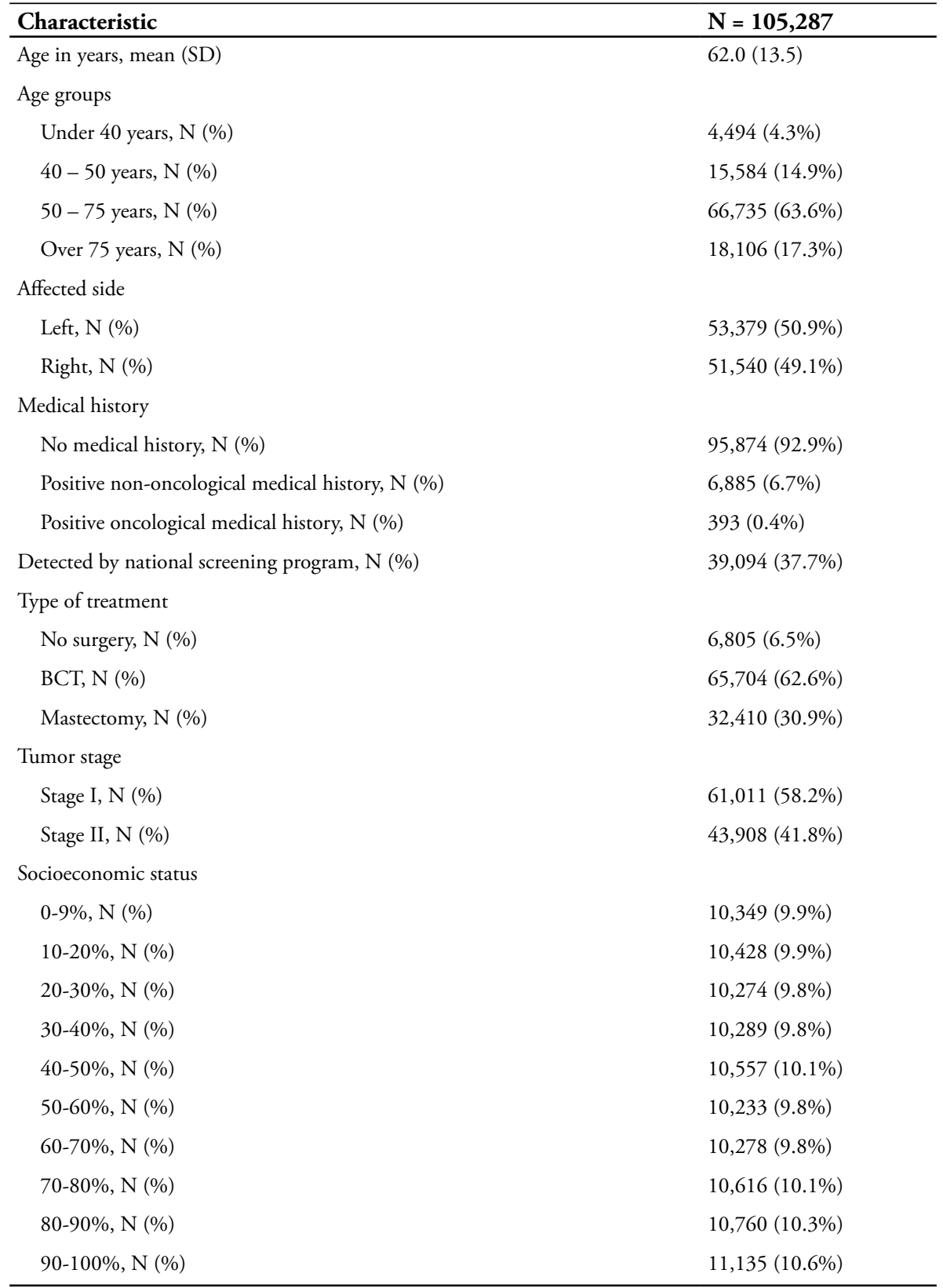

$\mathrm{SD}=$ Standard deviation, $\mathrm{N}=$ Number, BIRADS $=$ Breast Imaging Reporting and Data System, $\mathrm{BCT}=$ Breast conserving therapy. 


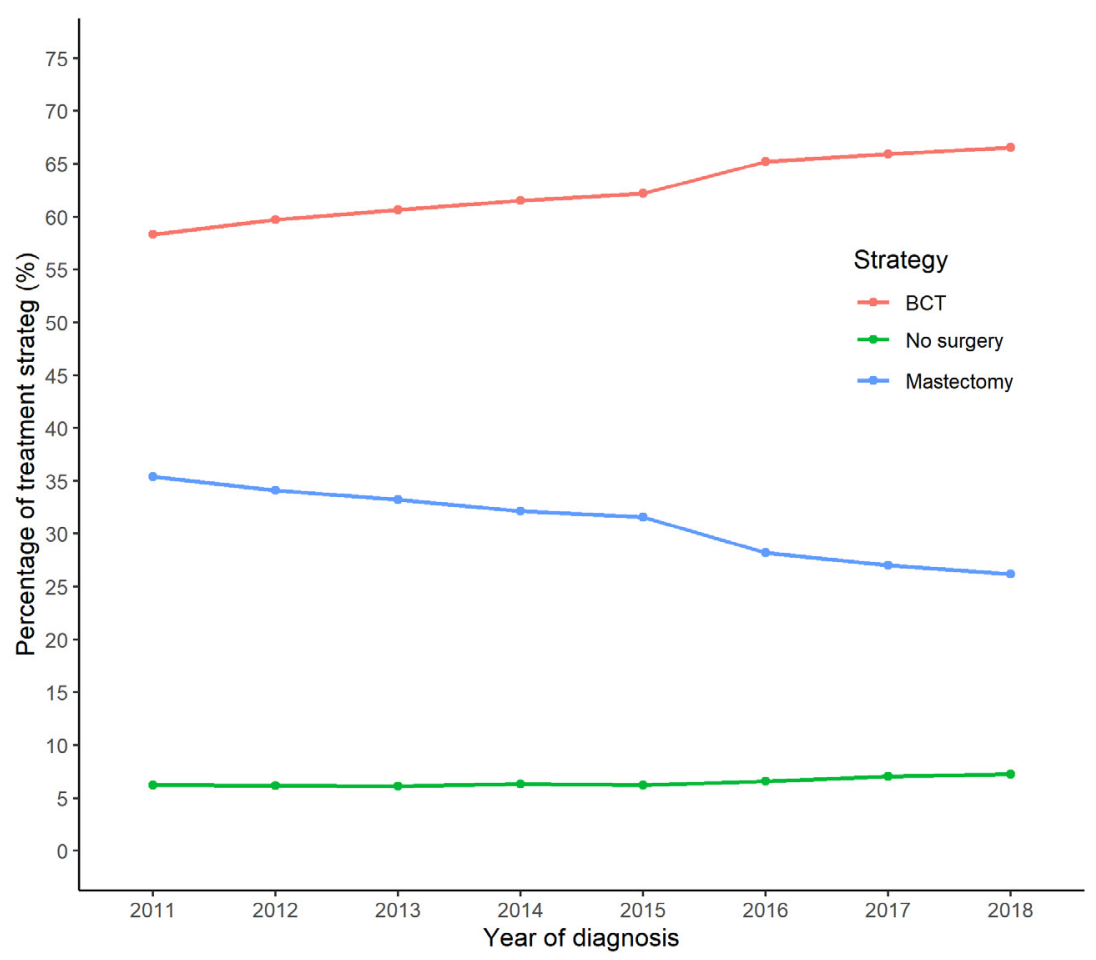

Figure 1. Trends of treatment strategy of new onset stage I and stage II breast cancer over time. BCT = Breast conserving therapy.

Table 2 shows the different characteristics of patients stratified for surgery-or-not. Patients not undergoing surgery were significantly older, and more often of higher stage and grade, and more often had HER2 negative and estrogen receptor positive tumors. Furthermore, patients undergoing surgery were more often triple negative $(3.8 \%)$ compared to patients not undergoing surgery (1.8\%). 9,465 (91.2\%) of the patients with the lowest SES underwent surgery which was significantly less than the $10,590(94.7 \%)$ of the patients with the highest SES. However, after correcting for patient and tumor characteristics in multivariable analysis, SES was no predictor for undergoing surgery, while age, triple negative receptor status and tumor stage (highest absolute z-value) played the most important role in determining surgery-or-not (Table 3). 
Table 2. Differences between no surgery and surgery of new onset breast cancer patients.

\begin{tabular}{|c|c|c|c|}
\hline Parameter & $\begin{array}{l}\text { No surgery } \\
n=6,840\end{array}$ & $\begin{array}{l}\text { Surgery } \\
n=98,447\end{array}$ & p-value \\
\hline Age in years, mean $(\mathrm{SD})$ & $82.1(11.1)$ & $60.6(12.6)$ & $<0.001$ \\
\hline Age groups & & & $<0.001$ \\
\hline Under 40 years, $\mathrm{N}(\%)$ & $50(0.7 \%)$ & $4,476(4.5 \%)$ & \\
\hline $40-50$ years, $\mathrm{N}(\%)$ & $139(2.0 \%)$ & $15,538(15.8 \%)$ & \\
\hline $50-75$ years, $N(\%)$ & $907(13.3 \%)$ & $66,013(67.1 \%)$ & \\
\hline Over 75 years, N (\%) & $5,744(84.0 \%)$ & $12,420(12.6 \%)$ & \\
\hline Detected during screening, N (\%) & $233(3.4 \%)$ & $39,370(40.0 \%)$ & $<0.001$ \\
\hline Socioeconomic status & & & $<0.001$ \\
\hline $0-9 \%, \mathrm{~N}(\%)$ & $917(8.8 \%)$ & $9,465(91.2 \%)$ & \\
\hline $10-20 \%, \mathrm{~N}(\%)$ & $774(7.4 \%)$ & $9,700(92.6 \%)$ & \\
\hline $20-30 \%, N(\%)$ & $717(7.0 \%)$ & $9,588(93.0 \%)$ & \\
\hline $30-40 \%, \mathrm{~N}(\%)$ & $665(6.4 \%)$ & $9,662(93.6 \%)$ & \\
\hline $40-50 \%, N(\%)$ & $675(6.4 \%)$ & $9,912(93.6 \%)$ & \\
\hline $50-60 \%, N(\%)$ & $685(6.7 \%)$ & $9,587(93.3 \%)$ & \\
\hline $60-70 \%, N(\%)$ & $623(6.0 \%)$ & $9,688(94.0 \%)$ & \\
\hline $70-80 \%, \mathrm{~N}(\%)$ & $594(5.6 \%)$ & $10,056(94.4 \%)$ & \\
\hline $80-90 \%, \mathrm{~N}(\%)$ & $595(5.5 \%)$ & $10,199(94.5 \%)$ & \\
\hline $90-100 \%, \mathrm{~N}(\%)$ & $595(5.3 \%)$ & $10,590(94.7 \%)$ & \\
\hline Tumor stage & & & $<0.001$ \\
\hline Stage I, N (\%) & $2,394(3.9 \%)$ & $58,617(96.1 \%)$ & \\
\hline Stage II, N (\%) & $4,446(10.0 \%)$ & $39,830(90.0 \%)$ & \\
\hline Medical history & & & $<0.001$ \\
\hline No medical history, N (\%) & $6,562(95.9 \%)$ & $91,299(92.7 \%)$ & \\
\hline Positive non-oncological medical history, N (\%) & $247(3.6 \%)$ & $6,774(6.9 \%)$ & \\
\hline Positive oncological medical history, N (\%) & $31(0.5 \%)$ & $374(0.4 \%)$ & \\
\hline \multicolumn{4}{|l|}{ Hormone receptor status } \\
\hline Her2 receptor negative, $\mathrm{N}(\%)$ & $6,331(92.6 \%)$ & $86,808(88.2 \%)$ & $<0.001$ \\
\hline Progesterone receptor positive, N (\%) & $4,636(67.8 \%)$ & $66,854(67.9 \%)$ & 0.831 \\
\hline Estrogen receptor positive, N (\%) & $5,820(85.1 \%)$ & $81,244(82.4 \%)$ & $<0.001$ \\
\hline Triple negative & $128(1.8 \%)$ & $3,757(3.8 \%)$ & $<0.001$ \\
\hline
\end{tabular}




\begin{tabular}{|c|c|c|c|}
\hline Parameter & $\begin{array}{l}\text { No surgery } \\
n=6,840\end{array}$ & $\begin{array}{l}\text { Surgery } \\
\mathrm{n}=98,447\end{array}$ & p-value \\
\hline Tumor grade & & & $<0.001$ \\
\hline Low grade, $\mathrm{N}(\%)$ & $1,910(27.9 \%)$ & $24,757(25.1 \%)$ & \\
\hline Intermediate grade, $\mathrm{N}(\%)$ & $3,505(51.2 \%)$ & $47,590(48.3 \%)$ & \\
\hline High grade, $\mathrm{N}(\%)$ & $1,425(20.8 \%)$ & $26,100(26.5 \%)$ & \\
\hline Histological tumor type & & & $<0.001$ \\
\hline No special type, N (\%) & $5,198(76 \%)$ & $79,460(80.7 \%)$ & \\
\hline Lobular (ILC), N (\%) & $1,072(15.7 \%)$ & $11,536(11.7 \%)$ & \\
\hline Both, N (\%) & $91(1.3 \%)$ & $3,041(3.1 \%)$ & \\
\hline Mucinous, N (\%) & $300(4.4 \%)$ & $1,756(1.8 \%)$ & \\
\hline Medullary, N (\%) & $1(0.0 \%)$ & $594(0.6 \%)$ & \\
\hline Tubular, N (\%) & $18(0.3 \%)$ & $898(0.9 \%)$ & \\
\hline Other, N (\%) & $160(2.3 \%)$ & $1,162(1.2 \%)$ & \\
\hline Hospital volume & & & 0.395 \\
\hline Low volume & $662(9.7 \%)$ & $9,166(9.3 \%)$ & \\
\hline Average volume & $1,649(24.1 \%)$ & $24,302(24.7 \%)$ & \\
\hline High volume & $4,529(66.2 \%)$ & $64,979(66.0 \%)$ & \\
\hline Year of diagnosis & & & $<0.001$ \\
\hline 2011, N (\%) & $792(6.2 \%)$ & $11,908(93.8 \%)$ & \\
\hline 2012, N (\%) & $798(6.1 \%)$ & $12,180(93.9 \%)$ & \\
\hline 2013, N (\%) & $800(6.1 \%)$ & $12,290(93.9 \%)$ & \\
\hline 2014, N (\%) & $833(6.3 \%)$ & $12,332(93.7 \%)$ & \\
\hline 2015, N (\%) & $815(6.2 \%)$ & $12,329(93.8 \%)$ & \\
\hline 2016, N (\%) & $870(6.6 \%)$ & $12,324(93.4 \%)$ & \\
\hline 2017, N (\%) & $961(7.0 \%)$ & $12,700(93.0 \%)$ & \\
\hline 2018, N (\%) & $971(7.3 \%)$ & $12,384(92.7 \%)$ & \\
\hline
\end{tabular}

$\mathrm{BCT}$ = breast conserving therapy, $\mathrm{N}=$ Number, $\mathrm{SD}=$ Standard deviation, BIRADS = Breast Imaging Reporting and Data System, ILS = Invasive lobular carcinoma 
Table 3. Multivariate regression analysis factors influencing non surgical therapy versus vs surgery.

\begin{tabular}{|c|c|c|c|c|c|}
\hline Parameter & $\begin{array}{l}\text { Estimate } \\
(\beta)\end{array}$ & OR (95\% CI) & Standard error & $Z$ value & P-value \\
\hline SES (per 10\% stratum) & 0.010 & $1.01(1.00-1.02)$ & 0.005 & 1.877 & 0.060 \\
\hline Age (years) & -0.169 & $0.84(0.84-0.85)$ & 0.002 & -94.497 & $<0.001$ \\
\hline Year of treatment & -0.026 & $0.97(0.96-0.99)$ & 0.007 & -3.953 & $<0.001$ \\
\hline \multicolumn{6}{|l|}{ Hospital volume } \\
\hline Small volume & NA & 1.00 (reference) & NA & NA & NA \\
\hline Average volume & 0.010 & $1.01(0.90-1.13)$ & 0.058 & 0.172 & 0.863 \\
\hline Large volume & -0.101 & $0.90(0.82-1.00)$ & 0.053 & -1.916 & 0.055 \\
\hline \multicolumn{6}{|l|}{ Tumor grade } \\
\hline Low grade & NA & 1.00 (reference) & NA & NA & NA \\
\hline Intermediate grade & 0.092 & $1.1(1.02-1.18)$ & 0.038 & 2.439 & 0.015 \\
\hline High grade & 0.375 & $1.45(1.32-1.60)$ & 0.049 & 7.700 & $<0.001$ \\
\hline \multicolumn{6}{|l|}{ Histological subtype } \\
\hline Ductal carcinoma & NA & 1.00 (reference) & NA & NA & NA \\
\hline ILC & -0.031 & $0.97(0.89-1.05)$ & 0.043 & -0.723 & 0.470 \\
\hline Both & 0.697 & $2.01(1.59-2.53)$ & 0.119 & 5.859 & $<0.001$ \\
\hline Mucinous & 0.232 & $1.26(1.08-1.47)$ & 0.078 & 2.964 & 0.003 \\
\hline Medullary & 3.040 & $20.89(2.92-149.49)$ & 1.004 & 3.028 & 0.002 \\
\hline Tubular & 0.400 & $1.49(0.90-2.47)$ & 0.257 & 1.556 & 0.120 \\
\hline Other & 0.027 & $1.03(0.83-1.27)$ & 0.107 & 0.254 & 0.799 \\
\hline \multicolumn{6}{|l|}{ Hormone receptor status } \\
\hline Her2 receptor & -0.136 & $0.87(0.78-0.98)$ & 0.057 & -2.376 & 0.017 \\
\hline $\begin{array}{l}\text { Progesterone receptor } \\
\text { positive }\end{array}$ & 0.060 & $1.06(0.98-1.15)$ & 0.040 & 1.506 & 0.132 \\
\hline Estrogen receptor positive & -0.063 & $0.94(0.84-1.05)$ & 0.054 & -1.148 & 0.251 \\
\hline Triple negative & 0.678 & $1.97(1.53-2.54)$ & 0.129 & 5.25 & $<0.001$ \\
\hline \multicolumn{6}{|l|}{ Patient history } \\
\hline No history of disease & NA & 1.00 (reference) & NA & NA & NA \\
\hline $\begin{array}{l}\text { Non-oncological } \\
\text { history }\end{array}$ & 0.354 & $1.42(1.23-1.64)$ & 0.073 & 4.824 & $<0.001$ \\
\hline Oncological history & 0.086 & $1.09(0.72-1.64)$ & 0.21 & 0.409 & 0.683 \\
\hline \multicolumn{6}{|l|}{ Tumor stage } \\
\hline Stage I tumor & NA & 1.00 (reference) & NA & NA & NA \\
\hline Stage II tumor & -0.609 & $0.54(0.51-0.58)$ & 0.032 & -18.999 & $<0.001$ \\
\hline
\end{tabular}

$\mathrm{OR}=$ odds ratio, $\mathrm{CI}=$ confidence interval, $\mathrm{SES}=$ socioeconomic status, ILC = invasive lobular carcinoma, NA $=$ not applicable, $\mathrm{NAN}=$ not a number 
Stratified by type of breast cancer surgery (table 4) patients that underwent mastectomy were slightly, but significantly, older and had lower SES. Additionally, patients with lower tumor stage more often received BCT. Furthermore, patients undergoing mastectomy were more often HER2 negative and estrogen receptor positive compared to BCT counterparts. Moreover, increasing tumor grade and triple negative receptor status was associated to an increased likelihood of undergoing mastectomy. After correcting for patient and tumor characteristics, SES remained a significant predictor for type of surgery where breast cancer patients with higher SES were significantly more likely to undergo BCT (Table 5).

Table 4. Differences between BCT and mastectomy of new onset breast cancer patients.

\begin{tabular}{|c|c|c|c|}
\hline & BCT $n=65,888$ & Mastectomy n=32,559 & p-value \\
\hline Age in years, mean $(S D)$ & $60.4(11.3)$ & $60.7(14.7)$ & 0.001 \\
\hline \multicolumn{4}{|l|}{ Age groups } \\
\hline Under 40 years, $\mathrm{N}(\%)$ & $2,155(3.3 \%)$ & $2,321(7.1 \%)$ & \\
\hline $40-50$ years, $\mathrm{N}(\%)$ & $9,510(14.4 \%)$ & $6,028(18.5 \%)$ & \\
\hline $50-75$ years, $\mathrm{N}(\%)$ & $48,393(73.4 \%)$ & $17,620(54.1 \%)$ & \\
\hline Over 75 years, $\mathrm{N}(\%)$ & $5,830(8.8 \%)$ & $6,590(20.2 \%)$ & \\
\hline Socioeconomic status & & & $<0.001$ \\
\hline $0-9 \%, N(\%)$ & $6,067(64.1 \%)$ & $3,398(35.9 \%)$ & \\
\hline $10-20 \%, \mathrm{~N}(\%)$ & $6,290(64.8 \%)$ & $3,410(35.2 \%)$ & \\
\hline $20-30 \%, \mathrm{~N}(\%)$ & $6,270(65.4 \%)$ & $3,318(34.6 \%)$ & \\
\hline $30-40 \%, \mathrm{~N}(\%)$ & $6,448(66.7 \%)$ & $3,214(33.3 \%)$ & \\
\hline $40-50 \%, N(\%)$ & $6,689(67.5 \%)$ & $3,223(32.5 \%)$ & \\
\hline $50-60 \%, N(\%)$ & $6,466(67.4 \%)$ & $3,121(32.6 \%)$ & \\
\hline $60-70 \%, \mathrm{~N}(\%)$ & $6,558(67.7 \%)$ & $3,130(32.3 \%)$ & \\
\hline $70-80 \%, \mathrm{~N}(\%)$ & $6,840(68.0 \%)$ & $3,216(32.0 \%)$ & \\
\hline $80-90 \%, \mathrm{~N}(\%)$ & $7,075(69.4 \%)$ & $3,124(30.6 \%)$ & \\
\hline $90-100 \%, \mathrm{~N}(\%)$ & $7,185(67.8 \%)$ & $3,405(32.2 \%)$ & \\
\hline Tumor stage & & & $<0.001$ \\
\hline Stage I, N (\%) & $45,920(78.3 \%)$ & $12,697(21.7 \%)$ & \\
\hline Stage II, N (\%) & $19,968(50.1 \%)$ & $19,862(49.9 \%)$ & \\
\hline Medical history & & & $<0.001$ \\
\hline No medical history, N (\%) & $61,375(93.2 \%)$ & $29,766(91.4 \%)$ & \\
\hline $\begin{array}{l}\text { Positive non-oncological medical history, } \\
\text { N (\%) }\end{array}$ & $4,372(6.6 \%)$ & $2,546(7.8 \%)$ & \\
\hline Positive oncological medical history, N (\%) & $141(0.2 \%)$ & $247(0.8 \%)$ & \\
\hline
\end{tabular}




\begin{tabular}{|c|c|c|c|}
\hline & BCT $n=65,888$ & Mastectomy $n=32,559$ & p-value \\
\hline \multicolumn{4}{|l|}{ Hormone receptor status } \\
\hline Her2receptor negative, N (\%) & $59,057(59.6 \%)$ & $28,008(86.0 \%)$ & $<0.001$ \\
\hline Progesterone receptor positive, N (\%) & $45,924(69.7 \%)$ & $21,265(65.3 \%)$ & $<0.001$ \\
\hline Estrogen receptor positive, N (\%) & $55,295(83.9 \%)$ & $26,028(79.9 \%)$ & $<0.001$ \\
\hline Triple negative, N (\%) & $2,076(3.2 \%)$ & $1,681(5.2 \%)$ & $<0.001$ \\
\hline Tumor grade & & & $<0.001$ \\
\hline Low grade, $\mathrm{N}(\%)$ & $18,762(28.5 \%)$ & $6,014(18.5 \%)$ & \\
\hline Intermediate grade, $\mathrm{N}(\%)$ & $31,109(47.2 \%)$ & $16,415(50.4 \%)$ & \\
\hline High grade, N (\%) & $16,017(24.3 \%)$ & $10,130(31.1 \%)$ & \\
\hline Histological tumor type & & & $<0.001$ \\
\hline No special type, N (\%) & $55,100(83.6 \%)$ & $24,360(74.8 \%)$ & \\
\hline ILC, N (\%) & $6,192(9.4 \%)$ & $5,344(16.4 \%)$ & \\
\hline Both, N (\%) & $1,560(2.4 \%)$ & $1,481(4.5 \%)$ & \\
\hline Mucinous, N (\%) & $1,200(1.8 \%)$ & $556(1.7 \%)$ & \\
\hline Medullary, N (\%) & $399(0.6 \%)$ & $195(0.6 \%)$ & \\
\hline Tubular, N (\%) & $750(1.1 \%)$ & $148(0.5 \%)$ & \\
\hline Other, N (\%) & $687(1.0 \%)$ & $475(1.5 \%)$ & \\
\hline Neo-adjuvant therapy, N (\%) & $7,940(12.1 \%)$ & $4,893(15.0 \%)$ & $<0.001$ \\
\hline Adjuvant therapy, N (\%) & $64,576(98.0 \%)$ & $19,337(59.4 \%)$ & $<0.001$ \\
\hline Detected during screening & $31,574(47.9 \%)$ & $7,796(23.9 \%)$ & $<0.001$ \\
\hline Hospital volume & & & $<0.001$ \\
\hline Low volume & $5,825(8.8 \%)$ & $3,341(10.3 \%)$ & \\
\hline Average volume & $15,876(24.1 \%)$ & $8,426(25.9 \%)$ & \\
\hline High volume & $44,187(67.1 \%)$ & $20,792(63.9 \%)$ & \\
\hline Year of diagnosis & & & $<0.001$ \\
\hline 2011, N (\%) & $7,411(62.2 \%)$ & $4,497(37.8 \%)$ & \\
\hline 2012, N (\%) & $7,754(63.7 \%)$ & $4,426(36.3 \%)$ & \\
\hline 2013, N (\%) & $7,943(64.6 \%)$ & $4,347(35.4 \%)$ & \\
\hline 2014, N (\%) & $8,103(65.7 \%)$ & $4,229(34.3 \%)$ & \\
\hline 2015, N (\%) & $8,178(66.3 \%)$ & $4,151(33.7 \%)$ & \\
\hline 2016, N (\%) & $8,602(69.8 \%)$ & $3,722(30.2 \%)$ & \\
\hline 2017, N (\%) & $9,009(70.9 \%)$ & $3,691(29.1 \%)$ & \\
\hline 2018, N (\%) & $8,888(71.8 \%)$ & $3,496(28.2 \%)$ & \\
\hline
\end{tabular}

BCT = breast conserving therapy, $\mathrm{N}=$ Number, $\mathrm{SD}=$ Standard deviation, $\mathrm{ILC}=$ Invasive lobular carcinoma 
Table 5. Multivariate regression analysis factors influencing the likelihood of undergoing mastectomy compared BCT.

\begin{tabular}{|c|c|c|c|c|c|}
\hline Parameter & Estimate $(\beta)$ & OR (95\% CI) & $\begin{array}{l}\text { Standard } \\
\text { error }\end{array}$ & $\begin{array}{l}\mathrm{Z} \\
\text { value }\end{array}$ & P-value \\
\hline SES (per 10\% stratum) & -0.023 & $0.98(0.97-0.98)$ & 0.003 & -9.013 & $<0.001$ \\
\hline Age (years) & 0.004 & $1.00(1.00-1.01)$ & 0.001 & 7.758 & $<0.001$ \\
\hline Year of surgery & -0.073 & $0.93(0.92-0.94)$ & 0.003 & -22.659 & $<0.001$ \\
\hline \multicolumn{6}{|l|}{ Hospital volume } \\
\hline Small volume & NA & 1.00 (reference) & NA & NA & NA \\
\hline Average volume & 0.009 & $1.01(0.96-1.06)$ & 0.027 & 0.323 & 0.747 \\
\hline Large volume & -0.111 & $0.90(0.85-0.94)$ & 0.025 & -4.415 & $<0.001$ \\
\hline \multicolumn{6}{|l|}{ Tumor grade } \\
\hline Low grade & NA & 1.00 (reference) & NA & NA & NA \\
\hline Intermediate grade & 0.190 & $1.21(1.16-1.26)$ & 0.019 & 9.885 & $<0.001$ \\
\hline High grade & 0.270 & $1.31(1.25-1.37)$ & 0.023 & 11.649 & $<0.001$ \\
\hline \multicolumn{6}{|l|}{ Histological subtype } \\
\hline Ductal carcinoma & NA & 1.00 (reference) & NA & NA & NA \\
\hline ILC & 0.625 & $1.87(1.79-1.95)$ & 0.022 & 28.307 & $<0.001$ \\
\hline Both & 0.836 & $2.31(2.13-2.49)$ & 0.039 & 21.172 & $<0.001$ \\
\hline Mucinous & 0.07 & $1.07(0.96-1.19)$ & 0.055 & 1.271 & 0.204 \\
\hline Medullary & -0.055 & $0.95(0.79-1.14)$ & 0.093 & -0.588 & 0.556 \\
\hline Tubular & -0.193 & $0.82(0.69-0.99)$ & 0.093 & -2.083 & 0.037 \\
\hline Other & 0.271 & $1.31(1.16-1.49)$ & 0.064 & 4.217 & $<0.001$ \\
\hline \multicolumn{6}{|l|}{ Hormone receptor status } \\
\hline Her2 receptor negative & -0.128 & $0.88(0.83-0.93)$ & 0.027 & -4.775 & $<0.001$ \\
\hline Progesterone receptor positive & 0.019 & $1.02(0.98-1.06)$ & 0.020 & 0.928 & 0.353 \\
\hline Estrogen receptor positive & 0.002 & $1.00(0.95-1.06)$ & 0.028 & 0.058 & 0.954 \\
\hline Triple negative & 0.171 & $1.19(1.08-1.30)$ & 0.047 & 3.614 & $<0.001$ \\
\hline \multicolumn{6}{|l|}{ Patient Medical history } \\
\hline history of disease & NA & 1.00 (reference) & NA & NA & NA \\
\hline $\begin{array}{l}\text { Non-oncological medical } \\
\text { history }\end{array}$ & 0.258 & $1.29(1.23-1.37)$ & 0.028 & 9.339 & $<0.001$ \\
\hline Oncological medical history & 1.612 & $5.01(4.01-6.26)$ & 0.114 & 14.198 & $<0.001$ \\
\hline \multicolumn{6}{|l|}{ Tumor stage } \\
\hline Stage I tumor & NA & 1.00 (reference) & NA & NA & NA \\
\hline Stage II tumor & 1.231 & $3.42(3.33-3.53)$ & 0.015 & 82.180 & $<0.001$ \\
\hline
\end{tabular}

$\mathrm{NA}=$ not applicable, $\mathrm{OR}=$ odds ratio, $\mathrm{CI}=$ confidence interval, $\mathrm{SES}=$ socioeconomic status, $\mathrm{ILC}=$ invasive lobular carcinoma. 


\section{Discussion}

In this population-based study in a country where everyone has equal access to care, patients with newly diagnosed stage I or II breast cancer, patients with higher SES were significantly more likely to undergo BCT than mastectomy. SES did not affect whetheror-not patients underwent surgery-or-not, but older and higher tumor stage patients were less likely to undergo a surgical procedure. Furthermore, more BCT and fewer mastectomies are performed as the years go by.

The current study shows that for newly diagnosed stage I or II breast cancer patients, the higher the SES, the more likely it is that patients will undergo BCT, even in a country with equal access to care. This is in line with previous studies, regardless of whether there is universal income or not. ${ }^{17-19}$

Hospital with a lower breast cancer treating volume were less likely to perform BCT. This is in line with previous studies which reported that hospital volume affects different aspects of breast cancer treatment ${ }^{7,28}$. Additionally, reasons for these differences can be the preference of the clinician, unequal spread of SES within The Netherlands, age of patient, tumor grade, tumor stage and hormone receptor status ${ }^{7,8}$. Furthermore, a recent study showed that breast cancer patients with high SES are more likely to undergo postmastectomy reconstruction than their lower SES counterparts. ${ }^{29}$

The finding that SES did not play a role in whether-or-not patients underwent surgery is in contrast with countries with no universal healthcare systems, in which therapeutic choices are heavily influenced by income and health care insurance system. ${ }^{17,18}$ Age being the most important factor determining whether a patient underwent surgery is probably related to the fact that older patients are more likely to have co-morbidities with a higher risk of postoperative complications, leading to surgery less often being advised. ${ }^{30,31}$ This could also explain why newly diagnosed stage I or II breast cancer patients that did not undergo surgery were significantly older than patients who did undergo surgery (over 20 years on average).

Over time, more BCT procedures but fewer mastectomies were performed. This is probably due to increasingly favoring BCT over mastectomy with its higher complication rate along with serious cosmetic and psychological consequences, while have similar overall survival.${ }^{6,32,33}$

The current study shows that new onset stage I or II breast cancer patients the higher the SES, the more likely patients will undergo BCT. Furthermore, when adjusting for age, tumor characteristics and medical history, these differences remain. The current study shows that even in a country with equal access to care, SES does play a role in whether a patient receives BCT or mastectomy despite there being no differences in 
(cancer-free) survival. ${ }^{6,32,33}$ This is in line with previous studies, regardless of whether there is universal income or not. ${ }^{17-19}$ Additionally, this study shows that lower hospital breast cancer treating volume are less likely to perform BCT. This is in line with previous studies which reported that hospital volume affects different aspects of breast cancer treatment 7,28. Additionally, reasons for these differences can be the preference of the clinician, unequal spread of SES within the Netherlands, age of patient, tumor grade, tumor stage and hormone receptor status ${ }^{7,8}$. Furthermore, a recent study showed that breast cancer patients with high SES are more likely to undergo postmastectomy reconstruction than their lower SES counterparts. ${ }^{29}$

The present study has some limitations. Other factors, which are not recorded in the NCR, may also be determinants of surgery type such as race, ethnicity, health literacy, social environment, language, internet access and religion. However, these factors are known to be closely linked to SES. ${ }^{9,34,35}$ Nevertheless, more research is warranted to study the possible effects of cultural background on whether patients are given the same choices regarding breast cancer treatment when there is equal access to healthcare. Additionally, NCR does not record co-morbidities, which could also affect the therapeutic choice on whether or not to operate stage I or II breast cancer patients. Secondly, however, the current study does show that higher SES breast cancer patients are more likely to undergo BCT. The NCR does not have information about how well-informed patients are about their treatment options and if and to what extent shared decision making took place. Therefore, it would be interesting to study whether there also exist differences in information provision to women diagnosed with breast cancer regarding treatment options (non-surgical treatment, BCT or mastectomy) between hospitals and/or regions in the Netherlands and whether this is influenced by patients' SES or SES related factors. Moreover, maybe less access to patient information (due to e.g. language barrier, illiteracy, less access to internet) might contribute to the fact that patients with lower SES are less likely to undergo BCT when operated.

In conclusion, the current study shows that even in a country with equal access to healthcare, stage I or II breast cancer patients with lower SES were less likely to undergo BCT. Age and tumor stage, but not SES, were associated with undergoing surgery-or-not. 


\section{References}

1. Siegel RL, Miller KD, Jemal A. Cancer statistics, 2018. CA Cancer J Clin. 2018;68(1):7-30. doi:10.3322/ caac. 21442 [doi]

2. Vondeling GT, Menezes GL, Dvortsin EP, et al. Burden of early, advanced and metastatic breast cancer in The Netherlands. BMC Cancer. 2018;18(1):262-263. doi:10.1186/s12885-018-4158-3 [doi]

3. Koleva-Kolarova RG, Daszczuk AM, de Jonge C, et al. A modelling study to evaluate the costs and effects of lowering the starting age of population breast cancer screening. Maturitas. 2018;109:81-88. doi:S0378-5122(17)30543-1 [pii]

4. Agarwal S, Pappas L, Neumayer L, Kokeny K, Agarwal J. Effect of breast conservation therapy vs mastectomy on disease-specific survival for early-stage breast cancer. JAMA Surg. 2014;149(3):267-274. doi:10.1001/jamasurg.2013.3049 [doi]

5. Hartmann-Johnsen OJ, Karesen R, Schlichting E, Nygard JF. Survival is Better After Breast Conserving Therapy than Mastectomy for Early Stage Breast Cancer: A Registry-Based Follow-up Study of Norwegian Women Primary Operated Between 1998 and 2008. Ann Surg Oncol. 2015;22(12):38363845. doi:10.1245/s10434-015-4441-3 [doi]

6. van Maaren MC, de Munck L, de Bock GH, et al. 10 year survival after breast-conserving surgery plus radiotherapy compared with mastectomy in early breast cancer in the Netherlands: a population-based study. The LancetOncology. 2016;17(8):1158-1170. doi:S1470-2045(16)30067-5 [pii]

7. van Maaren MC, Strobbe LJA, Koppert LB, Poortmans PMP, Siesling S. Nationwide populationbased study of trends and regional variation in breast-conserving treatment for breast cancer. Br J Surg. 2018;105(13):1768-1777. doi:10.1002/bjs.10951

8. sociaal culureel planbureau. Regional differenes in socioeconomic status in the Netherlands [website in Dutch]. https:/www.volksgezondheidenzorg.info/onderwerp/sociaaleconomische-status/regionaalinternationaal/regionaal\#node-sociaaleconomische-status.

9. Adler NE, Boyce T, Chesney MA, et al. Socioeconomic status and health. The challenge of the gradient. Am Psychol. 1994;49(1):15-24.

10. Laaksonen M, Prattala R, Lahelma E. Sociodemographic determinants of multiple unhealthy behaviours. Scand J Public Health. 2003;31(1):37-43. doi:H7V3U63D37W9TTQT [pii]

11. Clegg LX, Reichman ME, Miller BA, et al. Impact of socioeconomic status on cancer incidence and stage at diagnosis: selected findings from the surveillance, epidemiology, and end results: National Longitudinal Mortality Study. Cancer Causes Control. 2009;20(4):417-435. doi:10.1007/s10552-008-9256-0 [doi]

12. Dohrenwend BP, Levav I, Shrout PE, et al. Socioeconomic status and psychiatric disorders: the causationselection issue. Science. 1992;255(5047):946-952.

13. Psaltopoulou T, Hatzis G, Papageorgiou N, Androulakis E, Briasoulis A, Tousoulis D. Socioeconomic status and risk factors for cardiovascular disease: Impact of dietary mediators. Hellenic J Cardiol. 2017;58(1):32-42. doi:S1109-9666(17)30040-4 [pii]

14. Saydah S, Lochner K. Socioeconomic status and risk of diabetes-related mortality in the U.S. Public Heal reports (Washington, DC 1974). 2010;125(3):377-388. doi:10.1177/003335491012500306 [doi]

15. Gu J, Groot G, Boden C, Busch A, Holtslander L, Lim H. Review of Factors Influencing Women’s Choice of Mastectomy Versus Breast Conserving Therapy in Early Stage Breast Cancer: A Systematic Review. Clin Breast Cancer. 2018;18(4):e539-e554. doi:S1526-8209(17)30691-2 [pii] 
16. van den Broek-Altenburg EM, Atherly AJ. The relation between selective contracting and healthcare expenditures in private health insurance plans in the United States. Health Policy (New York). 2020;124(2):174-182. doi:10.1016/j.healthpol.2019.12.008

17. Bhat S, Orucevic A, Woody C, Heidel RE, Bell JL. Evolving Trends and Influencing Factors in Mastectomy Decisions. Am Surg. 2017;83(3):233-238.

18. Churilla TM, Egleston B, Bleicher R, Dong Y, Meyer J, Anderson P. Disparities in the Local Management of Breast Cancer in the US according to Health Insurance Status. Breast J. 2017;23(2):169-176. doi:10.1111/tbj.12705 [doi]

19. Norredam M, Groenvold M, Petersen JH, Krasnik A. Effect of social class on tumour size at diagnosis and surgical treatment in Danish women with breast cancer. Soc Sci Med. 1998;47(11):1659-1663. doi:S0277953698002603 [pii]

20. van Weel C, Knottnerus JA, van Schayck OCP. Managing costs and access to healthcare in the Netherlands: impact on primary care. BMJ. April 2020:m1181. doi:10.1136/bmj.m1181

21. Rao AA, Feneis J, Lalonde C, Ojeda-Fournier H. A Pictorial Review of Changes in the BI-RADS Fifth Edition. Radiographics. 2016;36(3):623-639. doi:10.1148/rg.2016150178 [doi]

22. Li CI, Uribe DJ, Daling JR. Clinical characteristics of different histologic types of breast cancer. Br J Cancer. 2005;93(9):1046-1052. doi:10.1038/sj.bjc.6602787

23. Elston CW, Ellis IO. Pathological prognostic factors in breast cancer. I. The value of histological grade in breast cancer: experience from a large study with long-term follow-up. Histopathology. 1991;19(5):403410. doi:10.1111/j.1365-2559.1991.tb00229.x

24. Lagendijk M, van Maaren MC, Saadatmand S, et al. Breast conserving therapy and mastectomy revisited: Breast cancer-specific survival and the influence of prognostic factors in 129,692 patients. Int J cancer. 2018;142(1):165-175. doi:10.1002/ijc.31034 [doi]

25. de Ligt KM, van Bommel ACM, Schreuder K, et al. The effect of being informed on receiving immediate breast reconstruction in breast cancer patients. Eur J Surg Oncol. 2018;44(5):717-724. doi:10.1016/j. ejso.2018.01.226

26. Beaulieu-Jones BK, Lavage DR, Snyder JW, Moore JH, Pendergrass SA, Bauer CR. Characterizing and Managing Missing Structured Data in Electronic Health Records: Data Analysis. JMIR Med informatics. 2018;6(1):e11. doi:10.2196/medinform.8960 [doi]

27. Zhang Z. Multiple imputation with multivariate imputation by chained equation (MICE) package. Ann Transl Med. 2016;4(2):30-5839.2015.12.63. doi:10.3978/j.issn.2305-5839.2015.12.63 [doi]

28. van Maaren MC, le Cessie S, Strobbe LJA, Groothuis-Oudshoorn CGM, Poortmans PMP, Siesling S. Different statistical techniques dealing with confounding in observational research: measuring the effect of breast-conserving therapy and mastectomy on survival. J Cancer Res Clin Oncol. 2019;145(6):14851493. doi:10.1007/s00432-019-02919-x

29. Filipe MD, Siesling S, Vriens MR, van Diest PJ, Witkamp AJ, Mureau MAM. Socioeconomic status significantly contributes to the likelihood of immediate postmastectomy breast reconstruction in the Netherlands: A nationwide study. Eur J Surg Oncol. September 2020. doi:10.1016/j.ejso.2020.09.016

30. Schlagnitweit P, Helfgott R, Theodosiadi S, et al. Impact of contemporary therapy- concepts on surgical morbidity in breast cancer patients: A retrospective single center analysis of 829 patients. Eur J Surg Oncol. 2020;46(8):1477-1483. doi:10.1016/j.ejso.2020.04.034

31. Divo MJ, Martinez CH, Mannino DM. Ageing and the epidemiology of multimorbidity. Eur Respir J. 2014;44(4):1055-1068. doi:10.1183/09031936.00059814 
32. Litiere S, Werutsky G, Fentiman IS, et al. Breast conserving therapy versus mastectomy for stage I-II breast cancer: 20 year follow-up of the EORTC 10801 phase 3 randomised trial. The LancetOncology. 2012;13(4):412-419. doi:10.1016/S1470-2045(12)70042-6 [doi]

33. van Maaren MC, de Munck L, Jobsen JJ, et al. Breast-conserving therapy versus mastectomy in T1$2 \mathrm{~N} 2$ stage breast cancer: a population-based study on 10-year overall, relative, and distant metastasis-free survival in 3071 patients. Breast Cancer Res Treat. 2016;160(3):511-521. doi:10.1007/s10549-0164012-8 [doi]

34. Ridgeway CL. Why Status Matters for Inequality. Am Sociol Rev. 2014;79(1):1-16. doi:10.1177/ 0003122413515997

35. Berger J, Norman RZ, Balkwell JW, Smith RF. Status Inconsistency in Task Situations: A Test of Four Status Processing Principles. Am Sociol Rev. 1992;57(6):843. doi:10.2307/2096127 


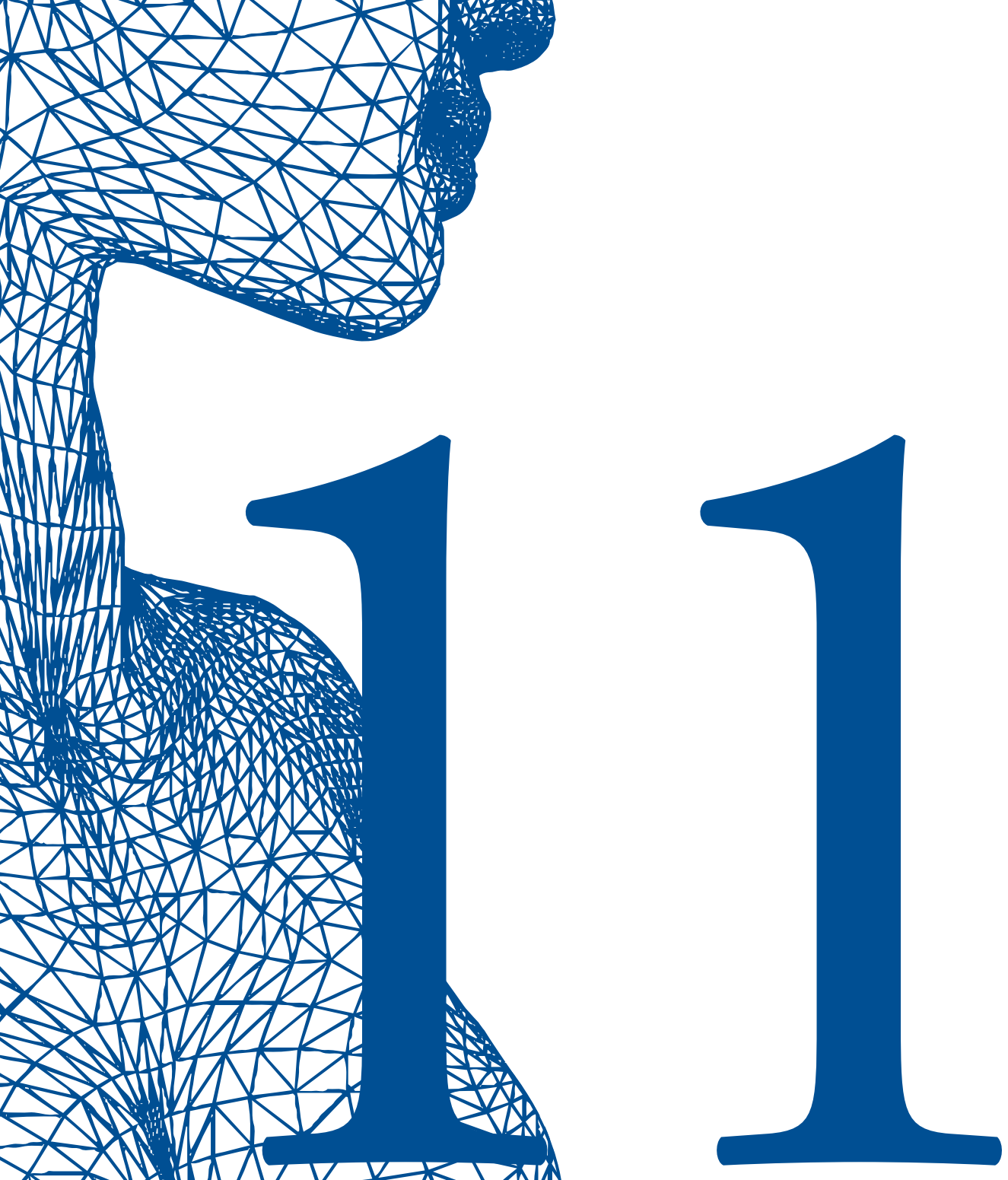




\section{Chapter 11}

Socioeconomic status significantly contributes to the likelihood of immediate postmastectomy breast reconstruction in the Netherlands: a nationwide study

P.J. van Diest

A.J. Witkamp 


\section{Abstract}

\section{Background}

Previous studies have shown that breast cancer patients with a low socioeconomic status (SES) are less likely to undergo postmastectomy immediate breast reconstruction (IBR). However, these studies were performed in countries with unequal access to healthcare. Therefore, the aim of this study was to investigate whether SES also contributes to the likelihood of receiving IBR in a country with equal access to healthcare.

\section{Materials and methods}

Patients with stage I or II breast cancer diagnosed between 2011 and 2018 who underwent mastectomy were selected from the Netherlands Cancer Registry. SES was calculated from the average incomes of each postal code which were divided into 10-deciles. Primary outcome was the effect of SES on the likelihood of receiving IBR, controlled for patient, tumour and hospital characteristics expressed as Odds Ratio (OR) with 95\% confidence interval (CI).

\section{Results}

Higher SES significantly increased the probability of undergoing postmastectomy IBR (OR 1.05 per 10\% SES stratum), just as larger hospital volume (average volume OR 1.89 and large volume 2.58), oestrogen positive tumours (OR 1.19) and neo-adjuvant therapy (OR 1.42). In contrast, factors significantly reducing the likelihood of receiving IBR were older age (OR 0.92 per year), stage II (OR 0.61 compared to stage I) and adjuvant therapy (OR 0.56).

\section{Conclusion}

Women with lower SES undergoing mastectomy were less likely to receive postmastectomy IBR. More research is warranted to study whether lifestyle factors associated with lower SES such as smoking and higher BMI, language barrier, illiteracy and less access to internet explain these differences. 


\section{Introduction}

Breast cancer is the most common cancer in women and the second most common cause of death due to cancer in women worldwide. ${ }^{1}$ There are approximately 17.000 new cases of breast cancer in the Netherlands every year and over 3.000 women of the Dutch population annually die due to breast cancer. ${ }^{2,3}$

Approximately $40 \%$ of patients with invasive breast cancer and $30 \%$ of patients with ductal carcinoma in situ (DCIS) undergo mastectomy in the Netherlands. ${ }^{4}$ To restore the breast contour, breast reconstruction may be performed either at the time of initial breast cancer surgery (immediate breast reconstruction, IBR) or as a delayed procedure some time later. IBR has positive effects on body image and psychosocial well-being and current guidelines recommend to offer the possibility of IBR to every patient with an indication for mastectomy. Nonetheless, a rather low mean IBR rate of $18 \%$ in patients undergoing mastectomy for invasive breast cancer was observed in The Netherlands with a substantial variation between Dutch hospitals. ${ }^{6}$ In previous studies, case-mix variation, ${ }^{6}$ hospital organizational factors, ${ }^{7}$ attitudes of clinicians towards IBR also taking risk factors for complications after IBR such as smoking and body mass index (BMI) into account, ${ }^{8}$ and information provision about IBR were identified as possible causes of this hospital variation. ${ }^{9}$

Previous studies from Denmark and the USA have found that women with lower socioeconomic status (SES) are less likely to undergo IBR after mastectomy. ${ }^{10,11}$ However, the Danish study is over 20 years old and in the USA there is no universal healthcare system, which means the insurance and financial reimbursement system of the USA leads to unequal insurance coverage of patients, related to their income and SES, which in turn heavily influences therapeutic choices. ${ }^{12-15}$

SES is a multi-layered system to stratify economic and social factors such as income, prestige and social status. ${ }^{16} \mathrm{SES}$ has been shown to be of influence in a wide array of diseases. For example, low SES has been associated with higher rates of diabetes, cardiovascular disease and many types of cancer in the western world and also psychiatric disorders are more often seen in patients with low SES. ${ }^{17-20}$ Moreover, high-risk behaviour (such as drugs, tobacco and alcohol abuse, high BMI) occurs more often in people with low SES and therefore contributes to an increased risk of the aforementioned diseases. ${ }^{21}$ Since SES plays an important role in the previously mentioned points, it is important to determine whether SES also relates to medical decisions such as the choice of IBR in countries with equal access to healthcare. Therefore, the aim of this study was to investigate whether SES contributes to the likelihood of receiving postmastectomy IBR in patients with stage I or II breast cancer in a country with equal access to healthcare, controlled for other patient, tumour and hospital characteristics which have been shown to affect the use of IBR. 


\section{Materials and methods}

\section{Study design and population}

In this nationwide population-based study, we selected breast cancer patients of the Netherlands Cancer Registry (NKR). The present study focused on primary stage I and II breast cancer patients who had undergone a mastectomy between January 1st, 2011 and December 31st, 2018. Only patients diagnosed with breast cancer for the first time were included in this study. If patients developed contralateral breast cancer, only the first diagnosis was included in this study.

\section{Definitions}

The NKR contains patient-, tumour- and treatment characteristics. Tumours are categorized according to the tumour, node and metastasis (TNM) classification system. ${ }^{22}$ Due to changes in the $\mathrm{N} 1$ category from the 5 th to the 6 th editions of the International Classification of Diseases for Oncology, we classified the number of positive lymph into $\mathrm{N}$ categories. Patients without lymph node involvement were classified as N0 and patients with 1-3 positive lymph nodes were classified as N1. TNM was converted to tumour stage (stage I or stage II). Histological subtype consisted in lobular, ductal, mucinous, medullary tubular, not specified or no special type. ${ }^{23}$ Tumour grade was divided into low, intermediate and high grade cancers. ${ }^{24}$

Only the use of IBR was registered and for the analyses the different types of breast reconstruction (implant-based with or without $\mathrm{ADM} / \mathrm{mesh}$, latissimus dorsi with implant, autologous) were also grouped together. In addition, the hospital where patients had received surgery was also recorded since some hospitals may favour IBR while other hospitals may not. Therefore, we stratified hospitals based on breast cancer patients operated per year; low $(<100)$, medium (100-149) and high volume $(>150)$, as described in previous studies. ${ }^{9}$ Furthermore, radiotherapy, hormone therapy, immune therapy and chemotherapy were grouped into adjuvant or neo-adjuvant therapy.

SES was determined using the average income of a household according to the four-digit postal code in the Netherlands at time of diagnosis and surgical procedure, and was defined according to the Dutch Bureau of Statistics (CBS). ${ }^{25}$ Furthermore, the average incomes of each postal code were divided into 10-deciles. 


\section{Outcomes}

Primary outcome was the effect of SES on the likelihood of receiving IBR after mastectomy. The primary outcome was controlled for other patient, tumour and hospital characteristics which have been shown to affect the use of IBR expressed as Odds Ratio (OR).

\section{Statistics}

Descriptive statistics were used to describe patient, tumour and treatment characteristics. Continuous data are described with mean along with standard deviation (SD), or with median and interquartile range (IQR), depending on whether or not the data were normally distributed. Mann-Whitney-U tests or Student's t tests were used to test differences between groups of not normally and normally distributed continuous data, respectively. Differences between categorical data were analysed with Chi-Square or Fisher's exact tests.

Since some data was missing during the study period, multiple imputation by chained equations (MICE) were performed using the MICE package in R. After comparing and correlating the missing to the non-missing data, it was concluded that the values were missing at random. The imputation was repeated 20 times, followed by application of Rubin's rule to combine parameter estimates and standard errors. ${ }^{26,27}$ Imputed data was later compared to the complete cases to determine validity of the imputation model. Subsequently, the imputed data was used for analyses.

Multivariate logistic regression analyses were performed to study the association between SES and the likelihood (quantified in odds ratio [OR] and $95 \%$ confidence interval [CI]) of receiving IBR in patients with stage 1 or 2 breast cancer. Possible confounding factors and effect modifiers to be considered are age at diagnosis, stage (1 or 2 ) and comorbidities. Two-sided P-values below 0.05 were considered statistically significant.

All calculations were performed using RStudio 1.2.5001 (with $\mathrm{R}$ version: $\times 64$ 3.6.3). Visualization of plots was performed using the ggplot 2 package. 


\section{Results}

Between 2011 and 2018, 105,423 patients were diagnosed with breast cancer in the NKR with new onset stage I or II breast cancer, of whom 32,559 patients had undergone mastectomy (Table 1). Mean age was 60.8 years (range, 18-101 years) and most patients $(17,620$ patients, $54.1 \%)$ were between 50 and 75 years at diagnosis. The national screening program detected breast cancer in $7726(23.9 \%)$ patients and $241(7.5 \%)$ patients had a positive oncological history other than breast cancer. A total of 12,697 (39.0\%) patients were diagnosed with new onset stage I breast cancer and 6096 (18.7\%) of all patients had received IBR after mastectomy. Finally, the incidence of breast cancer was evenly spread among the different strata of SES (Table 1). 
Table 1. Baseline characteristics of all new onset breast cancer patients diagnosed in the Netherlands between 2011 and 2018 who underwent mastectomy ( $\mathrm{N}=32,559$ patients).

\begin{tabular}{|c|c|}
\hline Age in years, mean $(\mathrm{SD})$ & $60.8(14.7)$ \\
\hline \multicolumn{2}{|l|}{ Age groups } \\
\hline Under 40 years, $\mathrm{N}(\%)$ & $2,321(7.1 \%)$ \\
\hline $40-50$ years, $\mathrm{N}(\%)$ & $6,028(18.5 \%)$ \\
\hline $50-75$ years, $\mathrm{N}(\%)$ & $17,620(54.1 \%)$ \\
\hline Over 75 years, $\mathrm{N}(\%)$ & $6,590(20.2 \%)$ \\
\hline \multicolumn{2}{|l|}{ Affected side } \\
\hline Left, N (\%) & $16,543(50.8 \%)$ \\
\hline Right, N (\%) & $16,013(49.2 \%)$ \\
\hline \multicolumn{2}{|l|}{ Medical history } \\
\hline No medical history, N (\%) & $29,291(91.5 \%)$ \\
\hline Positive non-oncological medical history, N (\%) & $2,471(7.7 \%)$ \\
\hline Positive oncological medical history, N (\%) & $241(0.8 \%)$ \\
\hline Detected by national screening program, N (\%) & $7,726(23.9 \%)$ \\
\hline \multicolumn{2}{|l|}{ Tumour stage } \\
\hline Stage I, N (\%) & $12,697(39.0 \%)$ \\
\hline Stage II, N (\%) & $19,862(61.0 \%)$ \\
\hline Immediate beast reconstruction, N (\%) & $6,096(18.7 \%)$ \\
\hline \multicolumn{2}{|l|}{ Socioeconomic status } \\
\hline $0-9 \%, \mathrm{~N}(\%)$ & $3,398(10.4 \%)$ \\
\hline $10-20 \%, \mathrm{~N}(\%)$ & $3,410(10.5 \%)$ \\
\hline $20-30 \%, \mathrm{~N}(\%)$ & $3,318(10.2 \%)$ \\
\hline $30-40 \%, \mathrm{~N}(\%)$ & $3,214(9.9 \%)$ \\
\hline $40-50 \%, \mathrm{~N}(\%)$ & $3,223(9.9 \%)$ \\
\hline $50-60 \%, \mathrm{~N}(\%)$ & $3,121(9.6 \%)$ \\
\hline $60-70 \%, \mathrm{~N}(\%)$ & $3,130(9.6 \%)$ \\
\hline $70-80 \%, \mathrm{~N}(\%)$ & $3,216(9.9 \%)$ \\
\hline $80-90 \%, \mathrm{~N}(\%)$ & $3,124(9.6 \%)$ \\
\hline $90-100 \%, \mathrm{~N}(\%)$ & $3,404(10.5 \%)$ \\
\hline
\end{tabular}

SD = Standard deviation, N = Number, BIRADS = Breast Imaging Reporting and Data System . 
Compared to patients without IBR, patients with IBR after mastectomy were significantly older (63.3 years vs 49.7 years, respectively) and significantly more often had a stage I tumour ( $47.4 \%$ vs $37.1 \%$, respectively; Table 2 ). Tumour grade did not differ significantly between the groups but tumour histology did vary significantly between patients who had received reconstruction and those who had not. Finally and most importantly, 486 $(14.3 \%)$ of the patients with the lowest SES had received IBR after mastectomy which was significantly less than the 875 (25.7\%) of the patients with the highest SES (Table 2).

Table 2. Differences between patients with and without immediate breast reconstruction.

\begin{tabular}{|c|c|c|c|}
\hline & No IBR $n=26,463$ & IBR $n=6,096$ & p-value \\
\hline Age in years, mean $(\mathrm{SD})$ & $63.3(14.4)$ & $49.7(10.6)$ & $<0.001^{*}$ \\
\hline Age groups & & & $<0.001 \S$ \\
\hline Under 40 years, $\mathrm{N}(\%)$ & $1,287(55.5 \%)$ & $1,034(44.5 \%)$ & \\
\hline $40-50$ years, $\mathrm{N}(\%)$ & $4,019(66.7 \%)$ & $2,009(33.3 \%)$ & \\
\hline $50-75$ years, $N(\%)$ & $14,605(82.9 \%)$ & $3,015(17.1 \%)$ & \\
\hline Over 75 years, $\mathrm{N}(\%)$ & $6,552(99.4 \%)$ & $38(0.6 \%)$ & \\
\hline Detected during screening, $\mathrm{N}(\%)$ & $6,461(24.4 \%)$ & $1,329(21.8 \%)$ & $<0.001 \S$ \\
\hline Socioeconomic status & & & $<0.001 \$$ \\
\hline $0-9 \%, \mathrm{~N}(\%)$ & $2,913(85.7 \%)$ & $486(14.3 \%)$ & \\
\hline $10-19 \%, \mathrm{~N}(\%)$ & $2,871(84.2 \%)$ & $539(15.8 \%)$ & \\
\hline $20-29 \%, N(\%)$ & $2,761(83.2 \%)$ & $557(16.8 \%)$ & \\
\hline $30-39 \%, \mathrm{~N}(\%)$ & $2,685(83.5 \%)$ & $529(16.5 \%)$ & \\
\hline $40-49 \%, \mathrm{~N}(\%)$ & $2,669(82.8 \%)$ & $554(17.2 \%)$ & \\
\hline $50-59 \%, \mathrm{~N}(\%)$ & $2,566(82.2 \%)$ & $555(17.8 \%)$ & \\
\hline $60-69 \%, \mathrm{~N}(\%)$ & $2,491(79.6 \%)$ & $639(20.4 \%)$ & \\
\hline $70-79 \%, \mathrm{~N}(\%)$ & $2,555(79.4 \%)$ & $661(20.6 \%)$ & \\
\hline $80-89 \%, \mathrm{~N}(\%)$ & $2,423(77.6 \%)$ & $701(22.4 \%)$ & \\
\hline $90-100 \%, \mathrm{~N}(\%)$ & $2,529(74.3 \%)$ & $875(25.7 \%)$ & \\
\hline Received neo-adjuvant therapy, N (\%) & $3,194(12.1 \%)$ & $1,699(27.9 \%)$ & $<0.001 \$$ \\
\hline Received adjuvant therapy, N (\%) & $12,708(48.0 \%)$ & $2,894(47.5 \%)$ & $0.448 \$$ \\
\hline Tumour stage & & & $<0.001 \$$ \\
\hline Stage I, N (\%) & $9,809(37.1 \%)$ & $2,888(47.4 \%)$ & \\
\hline Stage II, N (\%) & $16,654(62.9 \%)$ & $3,208(52.6 \%)$ & \\
\hline Medical history & & & $<0.001 \$$ \\
\hline No medical history, N (\%) & $24,385(92.1 \%)$ & $5,402(88.6 \%)$ & \\
\hline
\end{tabular}




\begin{tabular}{|c|c|c|c|}
\hline & No IBR $n=26,463$ & IBR $n=6,096$ & p-value \\
\hline $\begin{array}{l}\text { Positive non-oncological medical } \\
\text { history, } \mathrm{N}(\%)\end{array}$ & $1,865(7.0 \%)$ & $663(10.9 \%)$ & \\
\hline $\begin{array}{l}\text { Positive oncological medical history, } \\
\text { N (\%) }\end{array}$ & $213(0.8 \%)$ & $31(0.5 \%)$ & \\
\hline \multicolumn{4}{|l|}{ Tumour receptor status } \\
\hline Her2 positive, $\mathrm{N}(\%)$ & $22,766(86.0 \%)$ & $5,132(84.2 \%)$ & $<0.001 \S$ \\
\hline Progesterone positive, N (\%) & $17,033(64.4 \%)$ & $4,132(67.8 \%)$ & $<0.001 \S$ \\
\hline Oestrogen positive, N (\%) & $21,083(79.7 \%)$ & $4,883(80.1 \%)$ & $0.460 \$$ \\
\hline Tumour grade & & & $0.088 \$$ \\
\hline Low grade, N (\%) & $4,843(18.3 \%)$ & $1,131(18.6 \%)$ & \\
\hline Intermediate grade, $\mathrm{N}(\%)$ & $13,382(50.6 \%)$ & $3,129(51.3 \%)$ & \\
\hline High grade, N (\%) & $8,238(31.1 \%)$ & $1,836(30.1 \%)$ & \\
\hline Histological tumour type & & & $<0.001 \S$ \\
\hline No special type (ductal), N (\%) & $19,560(73.9 \%)$ & $4,800(78.7 \%)$ & \\
\hline Lobular (ILC), N (\%) & $4,545(17.2 \%)$ & $799(13.1 \%)$ & \\
\hline Both, N (\%) & $1,199(4.5 \%)$ & $282(4.6 \%)$ & \\
\hline Mucinous, N (\%) & $484(1.8 \%)$ & $72(1.2 \%)$ & \\
\hline Medullary, N (\%) & $147(0.6 \%)$ & $48(0.8 \%)$ & \\
\hline Tubular, N (\%) & $112(0.4 \%)$ & $36(0.6 \%)$ & \\
\hline Other, N (\%) & $416(1.6 \%)$ & $59(1.0 \%)$ & \\
\hline
\end{tabular}

BCT = breast conserving therapy, $\mathrm{N}=$ Number, $\mathrm{SD}=$ Standard deviation, BIRADS = Breast Imaging Reporting and Data System, ILS = Invasive lobular carcinoma, IBR = Immediate breast reconstruction.

* Two Sample t-test.

$\$$ Chi-square test.

Multivariate analysis showed that histological subtype, her2 receptor positivity, and progesterone receptor positivity were not significant predictors (Fig. 1 and Supplementary Table 1). Factors significantly increasing the probability of undergoing postmastectomy IBR were higher SES (OR 1.05 [1.04-1.06] per 10\% SES stratum), larger hospital volume (average volume OR 1.89 [1.64-2.18] and large volume 2.58 [2.26-2.94]), oestrogen positive tumours (OR 1.19 [1.06-1.33]) and neo-adjuvant therapy (OR 1.42 [1.31-1.55]. In contrast, factors significantly reducing the likelihood of receiving postmastectomy IBR were older age (OR 0.92 [0.92-0.92] per year), stage II (OR 0.61 [0.57-0.65] compared to stage I) and adjuvant therapy (OR $0.56[0.52-0.60]$ ). 


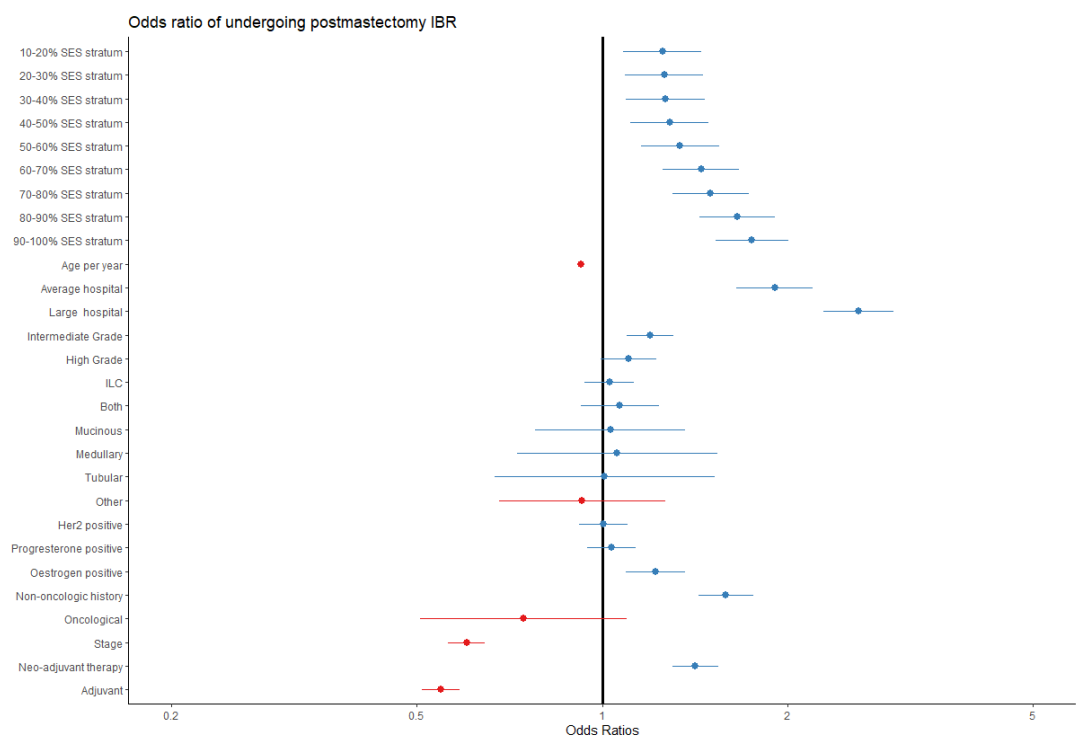

Fig. 1. Multivariate analysis of factors affecting the probability whether or not patients had undergone postmastectomy immediate breast reconstruction (IBR). SES = socioeconomic status, ILC $=$ invasive lobular carcinoma.

Most breast reconstructions were implant-based with or without ADM/mesh $(80.3 \%)$ followed by autologous (6.9\%) and combined techniques (latissimus dorsi and implant, $3.2 \%$ ). In $9.6 \%$ the reconstruction type was unknown. There was no difference in breast reconstruction type distribution between the SES strata (Fig. 2). 


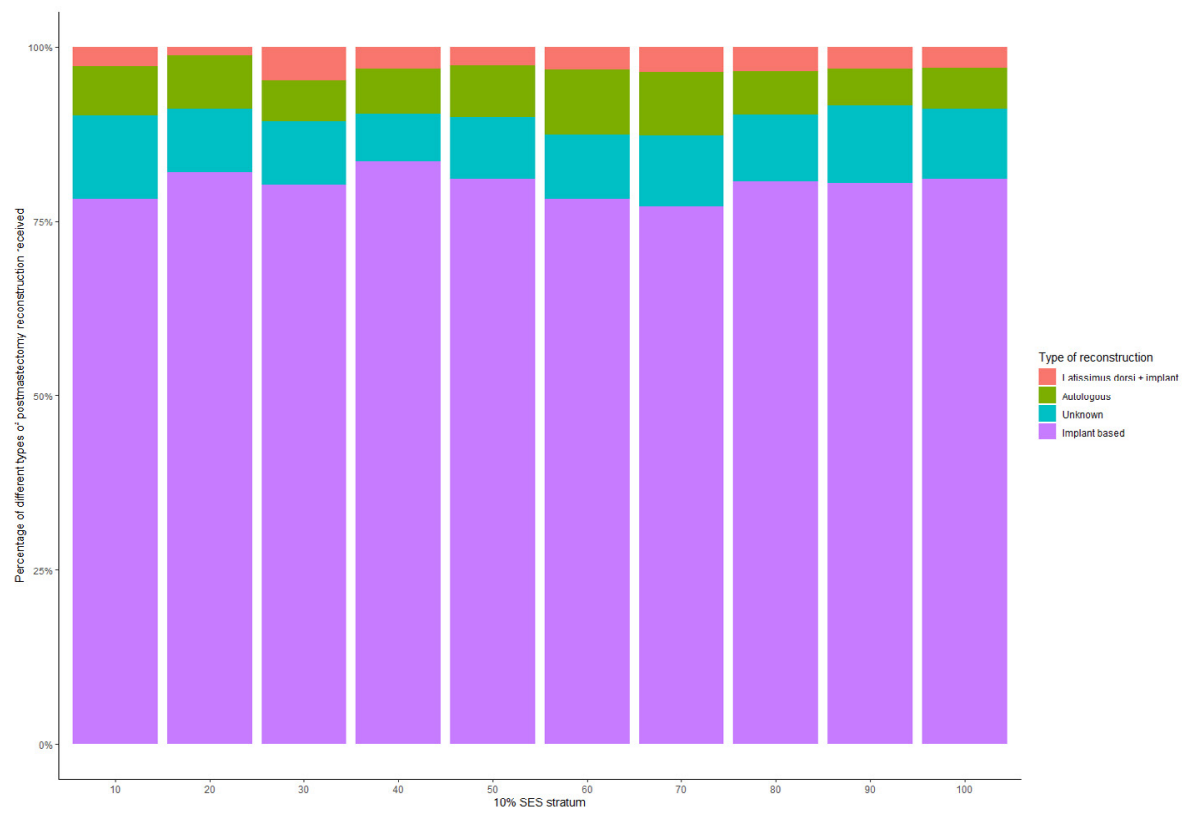

Fig. 2. Percentage of type of reconstruction per socioeconomic (SES) stratum.

In conclusion, SES remained a significant predictor for the likelihood of receiving IBR after multivariate logistic regression, controlling for age, tumour grade, tumour stage, (neo)adjuvant therapy, histological subtype, receptor status (oestrogen, progesterone and/ or her2) and medical history. 


\section{Discussion}

In this population-based study in a country where everyone has equal access to healthcare, patients with new onset stage I or II breast cancer undergoing mastectomy were more likely to undergo postmastectomy IBR with increasing SES. Other factors that increased the likelihood of undergoing IBR were larger hospital size in which the patient was treated, lower age and treatment with neo-adjuvant therapy and/or the absence of adjuvant therapy.

The current study showed that $14.3 \%$ of the patients from the lowest SES decile underwent postmastectomy IBR compared to $25.7 \%$ of the patients from the highest SES decile. Even after controlling for patient and tumour characteristics and hospital size, there was an OR of 1.75 (95\% CI of 1.53-2.01) of undergoing IBR in the highest SES decile compared to the lowest SES decile. This means that patients in the highest SES decile were $63.4 \%$ (95\% CI; 60.4-66.8\%) more likely to undergo IBR than patients in the lowest SES decile. This contributes to previous knowledge from studies which showed the effect of SES on the treatment of breast cancer. ${ }^{12,28,29}$ The current study is also in line with a recent Swedish study which showed that age and SES contribute to the likelihood of receiving postmastectomy IBR. ${ }^{30}$ In addition, the results of the current study confirm previously found results that SES affects the likelihood of undergoing postmastectomy IBR. ${ }^{10,11,31}$ In the Netherlands this means that most medical interventions including postmastectomy IBR are covered by national insurance for all the patients even for those of lower SES.

It was also found that hospital size was an important independent predictor of postmastectomy IBR. This is in line with previous studies which reported that hospital volume affects different aspects of breast cancer treatment. ${ }^{6,732-34}$ Compared to smaller hospitals in the Netherlands, larger hospitals often have plastic surgeons who are participating in the multidisciplinary team discussions and involved in the decision making of IBR. Moreover, they are better aware of all reconstruction possibilities and also take lifestyle factors such as higher BMI and smoking into account as well as the possibility of (neo)adjuvant therapies when recommending IBR. ${ }^{7,8}$ The current study confirms that the higher volume breast cancer treatment hospitals are more likely to perform postmastectomy IBR.

Age also appeared to be an important predictor of IBR. $44.5 \%$ of younger patients (under 40 years old) received IBR compared to only $0.6 \%$ of older patients (over 75 years old). Patients with stage II breast cancer who had received adjuvant therapy were less likely to undergo postmastectomy IBR while patients who had received neo-adjuvant therapy were more likely to undergo IBR. This is also in line with the results of previous studies which used data from a different national registry, ${ }^{6,9}$ giving us confidence in the reliability of our findings. 
The present study has some limitations. First, it showed that SES plays an important role whether or not patients received postmastectomy IBR. However, it is likely that other factors may also be important such as race, ethnicity and religious beliefs. However, these factors are known to be closely linked to SES. ${ }^{16,35,36}$ Therefore, more research is warranted to study the possible effects of cultural background on whether patients are given the same choices regarding postmastectomy IBR possibilities when there is equal access to healthcare. A previous study from the UK showed that there was variation between hospitals and regions in information provision regarding postmastectomy reconstruction in breast cancer patients. ${ }^{37}$ In the NKR we do not have information on cultural background, nor do we have information whether the possibility of IBR had been discussed with the patient. Therefore, it would be interesting to study whether there also exist differences in information provision to women undergoing mastectomy regarding IBR between hospitals and/or regions in the Netherlands and whether this is influenced by patients' SES. Moreover, maybe less access to patient information (due to e.g. language barrier, illiteracy, less access to internet) might contribute to the fact that patients with lower SES are less likely to undergo postmastectomy IBR. Therefore, it may be helpful to provide additional onco-psychological or social support to patients with lower SES.

In conclusion, the present study showed that, even in a country with equal access to healthcare, patients with lower SES were less likely to undergo postmastectomy IBR. More research is needed to determine why these differences exist. 


\section{References}

1. Siegel RL, Miller KD, Jemal A. Cancer statistics, 2018. CA Cancer J Clin. 2018;68(1):7-30. doi:10.3322/ caac. 21442 [doi]

2. Vondeling GT, Menezes GL, Dvortsin EP, et al. Burden of early, advanced and metastatic breast cancer in The Netherlands. BMC Cancer. 2018;18(1):262-263. doi:10.1186/s12885-018-4158-3 [doi]

3. Koleva-Kolarova RG, Daszczuk AM, de Jonge C, et al. A modelling study to evaluate the costs and effects of lowering the starting age of population breast cancer screening. Maturitas. 2018;109:81-88. doi:S0378-5122(17)30543-1 [pii]

4. van Bommel ACM, Spronk PER, Vrancken Peeters M-JTFD, et al. Clinical auditing as an instrument for quality improvement in breast cancer care in the Netherlands: The national NABON Breast Cancer Audit. J Surg Oncol. 2017;115(3):243-249. doi:10.1002/jso.24516

5. Mureau MAM, van der Hulst RRWJ, Woerdeman LAE, et al. Dutch breast reconstruction guideline. J Plast Reconstr Aesthetic Surg. 2018;71(3):290-304. doi:10.1016/j.bjps.2017.12.020

6. van Bommel ACM, Mureau MAM, Schreuder K, et al. Large variation between hospitals in immediate breast reconstruction rates after mastectomy for breast cancer in the Netherlands. J Plast Reconstr Aesthetic Surg. 2017;70(2):215-221. doi:10.1016/j.bjps.2016.10.022

7. Schreuder K, van Bommel ACM, de Ligt KM, et al. Hospital organizational factors affect the use of immediate breast reconstruction after mastectomy for breast cancer in the Netherlands. The Breast. 2017;34:96-102. doi:10.1016/j.breast.2017.05.011

8. van Bommel ACM, Schreuder K, Veenstra RK, et al. Discrepancies Between Surgical Oncologists and Plastic Surgeons in Patient Information Provision and Personal Opinions Towards Immediate Breast Reconstruction. Ann Plast Surg. 2018;81(4):383-388. doi:10.1097/SAP.0000000000001572

9. de Ligt KM, van Bommel ACM, Schreuder K, et al. The effect of being informed on receiving immediate breast reconstruction in breast cancer patients. Eur J Surg Oncol. 2018;44(5):717-724. doi:10.1016/j. ejso.2018.01.226

10. Bodilsen A, Christensen S, Christiansen P, Damsgaard TE, Zachariae R, Jensen AB. Socio-demographic, clinical, and health-related factors associated with breast reconstruction - A nationwide cohort study. The Breast. 2015;24(5):560-567. doi:10.1016/j.breast.2015.05.001

11. Siotos C, Lagiou P, Cheah MA, et al. Determinants of receiving immediate breast reconstruction: An analysis of patient characteristics at a tertiary care center in the US. Surg Oncol. 2020;34:1-6. doi:10.1016/j.suronc.2020.02.017

12. Gu J, Groot G, Boden C, Busch A, Holtslander L, Lim H. Review of Factors Influencing Women's Choice of Mastectomy Versus Breast Conserving Therapy in Early Stage Breast Cancer: A Systematic Review. Clin Breast Cancer. 2018;18(4):e539-e554. doi:S1526-8209(17)30691-2 [pii]

13. Scandlen G. Consumer-Driven Health Care: Just A Tweak Or A Revolution? Health Aff. 2005;24(6):15541558. doi: $10.1377 /$ hlthaff.24.6.1554

14. Murray CJL. The State of US Health, 1990-2010. JAMA. 2013;310(6):591. doi:10.1001/ jama.2013.13805

15. Dickman SL, Woolhandler S, Bor J, McCormick D, Bor DH, Himmelstein DU. Health Spending For Low-, Middle-, And High-Income Americans, 1963-2012. Health Aff. 2016;35(7):1189-1196. doi:10.1377/hlthaff.2015.1024 
16. Adler NE, Boyce T, Chesney MA, et al. Socioeconomic status and health. The challenge of the gradient. Am Psychol. 1994;49(1):15-24.

17. Clegg LX, Reichman ME, Miller BA, et al. Impact of socioeconomic status on cancer incidence and stage at diagnosis: selected findings from the surveillance, epidemiology, and end results: National Longitudinal Mortality Study. Cancer Causes Control. 2009;20(4):417-435. doi:10.1007/s10552-008-9256-0 [doi]

18. Dohrenwend BP, Levav I, Shrout PE, et al. Socioeconomic status and psychiatric disorders: the causationselection issue. Science. 1992;255(5047):946-952.

19. Psaltopoulou T, Hatzis G, Papageorgiou N, Androulakis E, Briasoulis A, Tousoulis D. Socioeconomic status and risk factors for cardiovascular disease: Impact of dietary mediators. Hellenic J Cardiol. 2017;58(1):32-42. doi:S1109-9666(17)30040-4 [pii]

20. Saydah S, Lochner K. Socioeconomic status and risk of diabetes-related mortality in the U.S. Public Heal reports (Washington, DC 1974). 2010;125(3):377-388. doi:10.1177/003335491012500306 [doi]

21. Laaksonen M, Prattala R, Lahelma E. Sociodemographic determinants of multiple unhealthy behaviours. Scand J Public Health. 2003;31(1):37-43. doi:H7V3U63D37W9TTQT [pii]

22. Rao AA, Feneis J, Lalonde C, Ojeda-Fournier H. A Pictorial Review of Changes in the BI-RADS Fifth Edition. Radiographics. 2016;36(3):623-639. doi:10.1148/rg.2016150178 [doi]

23. Li CI, Uribe DJ, Daling JR. Clinical characteristics of different histologic types of breast cancer. Br J Cancer. 2005;93(9):1046-1052. doi:10.1038/sj.bjc.6602787

24. Elston CW, Ellis IO. Pathological prognostic factors in breast cancer. I. The value of histological grade in breast cancer: experience from a large study with long-term follow-up. Histopathology. 1991;19(5):403410. doi:10.1111/j.1365-2559.1991.tb00229.x

25. Lagendijk M, van Maaren MC, Saadatmand S, et al. Breast conserving therapy and mastectomy revisited: Breast cancer-specific survival and the influence of prognostic factors in 129,692 patients. Int J cancer. 2018;142(1):165-175. doi:10.1002/ijc.31034 [doi]

26. Beaulieu-Jones BK, Lavage DR, Snyder JW, Moore JH, Pendergrass SA, Bauer CR. Characterizing and Managing Missing Structured Data in Electronic Health Records: Data Analysis. JMIR Med informatics. 2018;6(1):e11. doi:10.2196/medinform.8960 [doi]

27. Zhang Z. Multiple imputation with multivariate imputation by chained equation (MICE) package. Ann Transl Med. 2016;4(2):30-5839.2015.12.63. doi:10.3978/j.issn.2305-5839.2015.12.63 [doi]

28. Bhat S, Orucevic A, Woody C, Heidel RE, Bell JL. Evolving Trends and Influencing Factors in Mastectomy Decisions. Am Surg. 2017;83(3):233-238.

29. Churilla TM, Egleston B, Bleicher R, Dong Y, Meyer J, Anderson P. Disparities in the Local Management of Breast Cancer in the US according to Health Insurance Status. Breast J. 2017;23(2):169-176. doi: $10.1111 /$ tbj. 12705 [doi]

30. Unukovych D, Gümüscü R, Wärnberg F, et al. Breast reconstruction patterns from a Swedish nation-wide survey. Eur J Surg Oncol. May 2020. doi:10.1016/j.ejso.2020.04.030

31. Campbell I, Lao C, Blackmore T, et al. Surgical treatment of early stage breast cancer in the Auckland and Waikato regions of New Zealand. ANZ J Surg. 2018;88(12):1263-1268. doi:10.1111/ans.14840

32. de Boer AZ, Bastiaannet E, de Glas NA, et al. Effectiveness of radiotherapy after breast-conserving surgery in older patients with T1-2N0 breast cancer. Breast Cancer Res Treat. 2019;178(3):637-645. doi:10.1007/s10549-019-05412-8 
33. van Maaren MC, le Cessie S, Strobbe LJA, Groothuis-Oudshoorn CGM, Poortmans PMP, Siesling S. Different statistical techniques dealing with confounding in observational research: measuring the effect of breast-conserving therapy and mastectomy on survival. J Cancer Res Clin Oncol. 2019;145(6):14851493. doi:10.1007/s00432-019-02919-x

34. van Maaren MC, Strobbe LJA, Koppert LB, Poortmans PMP, Siesling S. Nationwide populationbased study of trends and regional variation in breast-conserving treatment for breast cancer. Br J Surg. 2018;105(13):1768-1777. doi:10.1002/bjs.10951

35. Ridgeway CL. Why Status Matters for Inequality. Am Sociol Rev. 2014;79(1):1-16. doi: $10.1177 / 0003122413515997$

36. Berger J, Norman RZ, Balkwell JW, Smith RF. Status Inconsistency in Task Situations: A Test of Four Status Processing Principles. Am Sociol Rev. 1992;57(6):843. doi:10.2307/2096127

37. Jeevan R, Cromwell DA, Browne JP, et al. Findings of a national comparative audit of mastectomy and breast reconstruction surgery in England. J Plast Reconstr Aesthetic Surg. 2014;67(10):1333-1344. doi:10.1016/j.bjps.2014.04.022 


\section{Supplementary table}

Supplementary table 1 . Multivariate regression analysis.

\begin{tabular}{|c|c|c|c|c|c|}
\hline Parameter & Estimate $(\beta)$ & OR (95\% CI) & Standard error & $Z$ value & P-value \\
\hline SES (per 10\% stratum) & 0.051 & $1.05(1.04-1.06)$ & 0.005 & 9.334 & $<0.001$ \\
\hline \multicolumn{6}{|l|}{ Hospital size } \\
\hline Low volume & NA & 1.00 (reference) & NA & NA & NA \\
\hline Average volume & 0.638 & $1.89(1.64-2.18)$ & 0.072 & 8.835 & $<0.001$ \\
\hline Large volume & 0.948 & $2.58(2.26-2.94)$ & 0.067 & 14.116 & $<0.001$ \\
\hline Age (years) & -0.080 & $0.92(0.92-0.92)$ & 0.001 & -55.04 & $<0.001$ \\
\hline \multicolumn{6}{|l|}{ Tumor grade } \\
\hline Low grade & NA & 1.00 (reference) & NA & NA & NA \\
\hline Intermediate grade & 0.142 & $1.15(1.06-1.26)$ & 0.044 & 3.213 & 0.001 \\
\hline High grade & -0.048 & $0.95(0.86-1.06)$ & 0.053 & -0.905 & 0.365 \\
\hline \multicolumn{6}{|l|}{ Histological subtype } \\
\hline Ductal carcinoma & NA & 1.00 (reference) & NA & NA & NA \\
\hline ILC & 0.012 & $1.01(0.92-1.11)$ & 0.047 & 0.251 & 0.802 \\
\hline Both & 0.057 & $1.06(0.91-1.23)$ & 0.075 & 0.765 & 0.444 \\
\hline Mucinous & 0.008 & $1.01(0.76-1.33)$ & 0.143 & 0.058 & 0.954 \\
\hline Medullary & 0.092 & $1.1(0.75-1.59)$ & 0.191 & 0.48 & 0.631 \\
\hline Tubular & -0.037 & $0.96(0.64-1.45)$ & 0.21 & -0.176 & 0.860 \\
\hline Other & -0.070 & $0.93(0.68-1.27)$ & 0.159 & -0.438 & 0.662 \\
\hline \multicolumn{6}{|l|}{ Hormone receptor status } \\
\hline Her2positive & -0.012 & $0.99(0.90-1.08)$ & 0.046 & -0.255 & 0.799 \\
\hline Progesterone positive & 0.007 & $1.01(0.92-1.10)$ & 0.046 & 0.145 & 0.885 \\
\hline Oestrogen positive & 0.172 & $1.19(1.06-1.33)$ & 0.057 & 3.039 & 0.002 \\
\hline \multicolumn{6}{|l|}{ Patient history } \\
\hline No history & NA & 1.00 (reference) & NA & NA & NA \\
\hline Non-oncological history & 0.458 & $1.58(1.42-1.75)$ & 0.053 & 8.719 & $<0.001$ \\
\hline Oncological history & -0.248 & $0.78(0.53-1.15)$ & 0.197 & -1.258 & 0.208 \\
\hline \multicolumn{6}{|l|}{ Tumour stage } \\
\hline Stage I tumour & NA & 1.00 (reference) & NA & NA & NA \\
\hline Stage II tumour & -0.498 & $0.61(0.57-0.65)$ & 0.036 & -13.95 & $<0.001$ \\
\hline
\end{tabular}




\begin{tabular}{llllll}
\hline Parameter & Estimate $(\beta)$ & OR $(\mathbf{9 5 \%} \mathbf{C I})$ & Standard error & $\mathbf{Z}$ value & P-value \\
\hline $\begin{array}{l}\text { Neo-adjuvant therapy } \\
\text { No Neo-adjuvant therapy }\end{array}$ & NA & 1.00 (reference) & NA & NA & NA \\
$\begin{array}{llll}\text { Neo-adjuvant therapy } \\
\text { Adjuvant therapy }\end{array}$ & 0.351 & $1.42(1.31-1.55)$ & 0.043 & 8.077 & $<0.001$ \\
$\begin{array}{l}\text { No adjuvant therapy } \\
\text { Adjuvant therapy }\end{array}$ & NA & 1.00 (reference) & NA & NA & NA \\
\hline
\end{tabular}

$\mathrm{OR}=$ odds ratio, $\mathrm{CI}=$ confidence interval, $\mathrm{SES}=$ socioeconomic status, $\mathrm{ILC}=$ invasive lobular carcinoma. 
Socioeconomic status significantly contributes to the likelihood of immediate postmastectomy breast reconstruction 


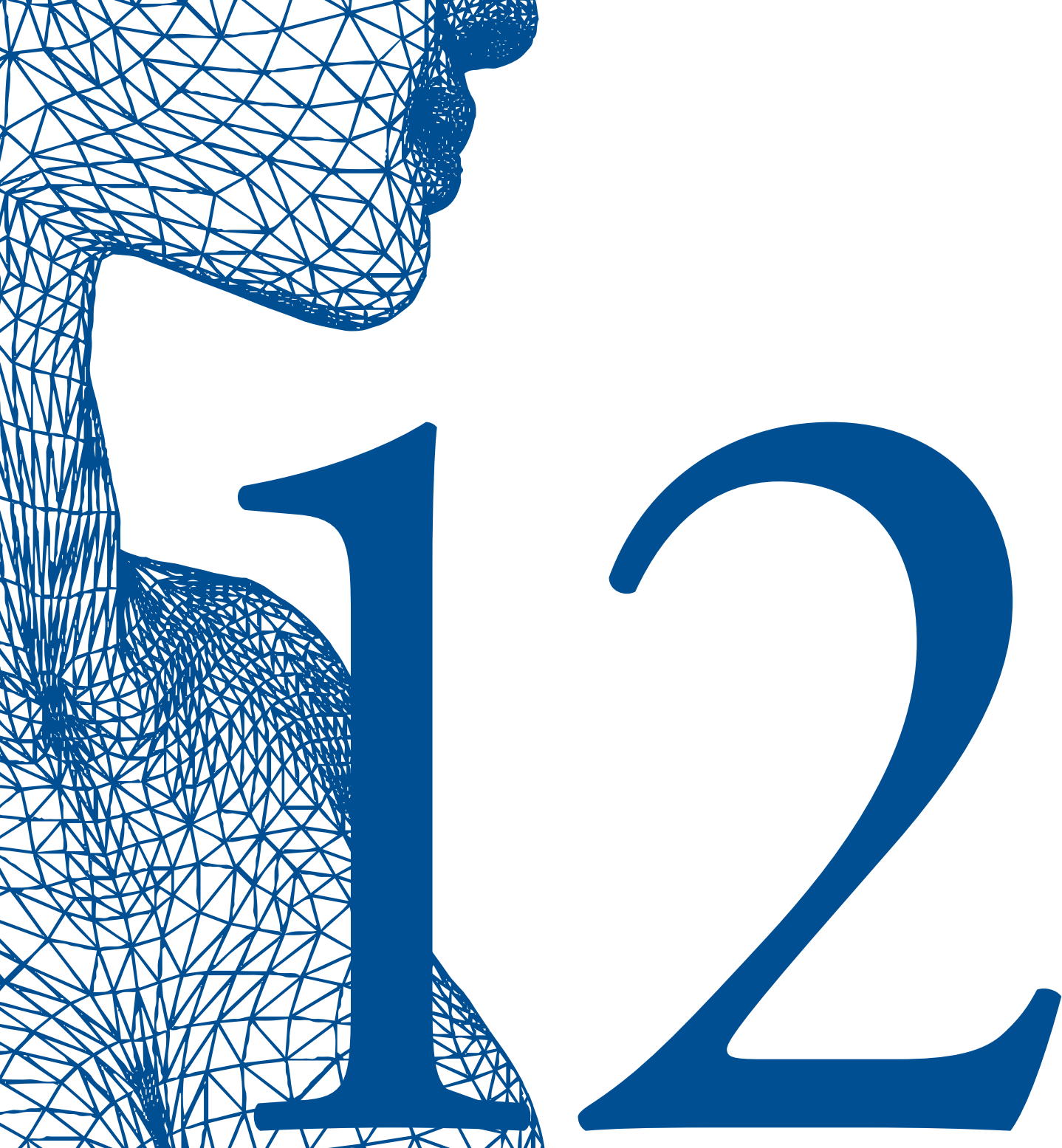




\section{Chapter 12}

\section{Effect of the COVID-19 pandemic on surgical breast cancer care in the Netherlands: a multicenter retrospective cohort study}

M.D. Filipe

D. van Deukeren M. Kip

A. Doeksen A. Pronk P.M. Verheijen J.T. Heikens

A.J. Witkamp M.C. Richir 


\section{Abstract}

\section{Background}

Coronavirus disease 2019 (COVID-19) has put a strain on regular healthcare worldwide. In the Netherlands, the national screening programs, including for breast cancer, were halted temporarily. This posed a challenge to breast cancer care, because $\sim 40 \%$ of cases are detected through national screening. Therefore, the aim of the present study was to evaluate the effects of the COVID-19 pandemic on the surgical care of patients with breast cancer in the Netherlands.

\section{Materials and Methods}

The present multicenter retrospective cohort study investigated the effects of COVID-19 on patients with breast cancer who had undergone surgery from March 9 to May 17, 2020. The primary endpoints were the number of surgical procedures performed during the study period, tumor characteristics, surgery type, and route of referral. The secondary endpoint was the incidence of postoperative complications during the study period.

\section{Results}

A total of 217 consecutive patients with breast cancer requiring surgery were included. We found an overall decrease in the number of patients with breast cancer who were undergoing surgery. The most significant decline was seen in surgery for T1-T2 and N0 tumors. A decline in the number of referrals from both the national screening program and general practitioners was observed. The incidence of postoperative complications remained stable during the study period.

\section{Conclusions}

The temporary halt of the national screening program for breast cancer resulted in fewer surgical procedures during the study period and a pronounced decrease in surgery of the lower tumor stages. 


\section{Introduction}

Coronavirus disease 2019 (COVID-19) is a highly infectious disease caused by acute respiratory syndrome coronavirus 2 (SARS-CoV-2) and responsible for the ongoing pandemic. ${ }^{1}$ SARS-CoV-2 can mostly be detected with polymerase chain reaction from oropharyngeal swabs. ${ }^{2}$ At the beginning of July 2020, > 11,000,000 cases and > 530,000 deaths had been confirmed worldwide. ${ }^{3}$

COVID-19 pandemic has posed a challenge to regular healthcare. ${ }^{4}$ Worldwide, measures to alleviate the burden on healthcare systems, such as the suspension of elective surgeries, were implemented. ${ }^{5}$ In the Netherlands, similar policies were implemented regarding oncologic care. If possible, elective surgeries were postponed, and the national screening programs for breast and colorectal cancer were temporarily halted starting March 16, $2020{ }^{4,6}$ This has led to a concerning decline in breast cancer diagnoses. ${ }^{4,7}$ Breast cancer is the most common cancer and the leading cause of cancer death in women worldwide. ${ }^{8}$ In the Netherlands, the incidence of breast cancer has been roughly 17,000 cases annually, with $\sim 40 \%$ detected through the national screening program. ${ }^{9,10}$ Surgical resection, with or without radiotherapy and/or systemic treatment, of the primary tumor is the treatment of choice for patients with new-onset breast cancer. ${ }^{11-13}$ The measures required to alleviate the burden on healthcare systems due to COVID-19 have strongly affected patients with breast cancer, especially the postponed surgeries and halting of national screening programs. $^{4,6}$

Recently, the number of breast cancer diagnoses has decreased by $\leq 50 \%$, which can be attributed to the temporary halting of national screening programs and/or fewer referrals from general practitioners (GPs). ${ }^{4}$ However, the consequences on surgical care for breast cancer are unknown. Therefore, the aim of the present study was to determine the effect of the COVID-19 pandemic on the surgical demand for breast cancer. 


\section{Materials and Methods}

\section{Patient Selection}

The present retrospective multicenter cohort study included all consecutive surgical patients treated from March 9 to May 17, 2020 in 5 hospitals in the Netherlands: University Medical Centre Utrecht, Ziekenhuis Rivierenland, St Antonius Ziekenhuis, Diakonessenhuis, and Meander Medical Centre. Consecutive patients were included if they were aged $>18$ years and had undergone breast cancer surgery. The ethics committee of all participating centers approved the present study and decided that patient informed consent was not required. The study is a part of the trial registered in the research registry (available at: www.researchregistry.com; unique identifying no., researchregistry5720).

\section{COVID-19 Diagnosis}

The included patients could have been tested for COVID-19 before or during their admission in the hospital. In addition, COVID-19-related symptoms were recorded. Reverse transcriptase polymerase chain reaction testing for SARS-CoV-2 was used at all the centers, in accordance with the European guidelines for analysis. 2 The genes used for analysis included the RdRp, E, and $\mathrm{N}$ genes. The E gene assay was used first, followed by confirmatory testing using the RdRp gene assay. For the present study, COVID-19related symptoms were defined as cough, fever, fatigue, chest pain, dyspnea, and other flu-like symptoms.

\section{Endpoints and Definitions}

The primary endpoints were the number of surgical procedures performed for breast cancer, TNM classification before surgery, type of surgical procedure, and initial referral (ie, the national screening program or the GP). The secondary endpoint was the incidence of postoperative complications. Additionally, we aimed to determine which factors influenced the risk of postoperative complications.

Surgical procedures were defined as breast-conserving therapy (BCT), mastectomy (with and without immediate reconstruction), and other procedures (ie, lymph node dissection, lymph node biopsy, repeat excision, scar excision).

The general condition of the patients before surgery was assessed using the American Society of Anesthesiologists classification. ${ }^{14}$ The severity of the complications was determined using to the Clavien-Dindo classification. ${ }^{15}$ Major complications were defined as ClavienDindo class $\geq$ III. The tumors were categorized using TNM classification system. ${ }^{16}$ Phyllodes tumors were not graded according to the TNM classification. ${ }^{17}$ Radiotherapy, 
antihormonal therapy, immunotherapy, and chemotherapy were classified as adjuvant and/or neoadjuvant therapy. Patient comorbidities included a history of cardiovascular disease, pulmonary disease, renal disease, and diabetes.

\section{Statistical Analysis}

Descriptive statistics were used to describe the patient and treatment characteristics. Continuous data are reported as the mean \pm standard deviation or median and interquartile range, depending on the distribution.

Multiple imputation by chained equations using the MICE package in R was performed to impute missing data. Missing data were compared with the available data to determine whether the data were missing at random. The imputation was repeated 10 times, followed by application of Rubin's rule to combine parameter estimates and standard errors. ${ }^{18,19}$ The imputed data were compared later with the complete data to determine the validity of the imputation model. The imputed data were used in the analyses. Two-sided $P$ values $<.05$ were considered statistically significant.

Multivariate logistic regression analyses were performed to study the risk of complications for patients who had undergone surgery. The odds ratio (OR) and $95 \%$ confidence intervals (CIs) were used to quantify the risk. Possible confounding factors and effect modifiers were age, body mass index, American Society of Anesthesiologists classification, type of breast surgery, number of comorbidities, TNM classification, week in which the surgery had been performed, symptoms associated with COVID-19, and testing for COVID-19.

All calculations were performed using RStudio, version 1.2.5001 (with $\mathrm{R}$ version, $\mathrm{x} 64$ 3.6.3). Visualization of the plots was performed using the ggplot 2 package. 


\section{Results}

\section{Patient Characteristics}

A total of 217 consecutive patients with breast cancer with a mean age of 62 years were included in the present study. Most of the patients had had a diagnosis of stage T1-T2 breast cancer $(81.7 \%)$ without lymph node involvement (N0; 71\%). In addition, 10 patients had had a phyllodes tumor. None of the included patients had had metastatic disease. Of the 217 patients, $61(28.1 \%)$ and 170 (78.3\%) had received neoadjuvant and adjuvant treatment, respectively. The surgery was BCT for 139 patients $(64.1 \%)$. Of the 217 patients, 21 had been tested for COVID-19 (9.7\%), none of whom tested positive. Complications occurred in 18 patients, of whom $8(3.7 \%)$ had developed major complications (Table 1). 
Table 1. Baseline Patient Characteristics $(\mathrm{n}=217)$

\begin{tabular}{|c|c|}
\hline Parameter & $\mathbf{N}=\mathbf{2 1 7}$ \\
\hline Age in years, mean $(\mathrm{SD})$ & $62.2(13.1)$ \\
\hline BMI in $\mathrm{kg} / \mathrm{m}^{2}$, median (IQR) & $23.3[23.0-30.4]$ \\
\hline \multicolumn{2}{|l|}{ ASA classification } \\
\hline ASA I, N (\%) & $47(21.7 \%)$ \\
\hline ASA II, N (\%) & $130(59.9 \%)$ \\
\hline ASA III, N (\%) & $40(18.4 \%)$ \\
\hline Diagnosis during national screening program, $\mathrm{N}(\%)$ & $55(25.7 \%)$ \\
\hline \multicolumn{2}{|l|}{ T stage } \\
\hline T0, N (\%) & $28(13.5 \%)$ \\
\hline $\mathrm{T} 1, \mathrm{~N}(\%)$ & $119(57.5 \%)$ \\
\hline $\mathrm{T} 2, \mathrm{~N}(\%)$ & $50(24.2 \%)$ \\
\hline T3, N (\%) & $6(2.9 \%)$ \\
\hline T4, N (\%) & $4(1.9 \%)$ \\
\hline T missing, $\mathrm{N}$ & 10 \\
\hline \multicolumn{2}{|l|}{ N stage } \\
\hline N0, N (\%) & $147(71.0 \%)$ \\
\hline N1, N (\%) & $49(23.7 \%)$ \\
\hline $\mathrm{N} 2, \mathrm{~N}(\%)$ & $7(3.4 \%)$ \\
\hline N3, N (\%) & $4(1.9 \%)$ \\
\hline $\mathrm{N}$ missing, $\mathrm{N}$ & 10 \\
\hline M stage & $0(0.0 \%)$ \\
\hline Neo-adjuvant therapy & $61(28.1 \%)$ \\
\hline Adjuvant therapy & $170(78.3 \%)$ \\
\hline \multicolumn{2}{|l|}{ Type of surgery } \\
\hline BCT, N (\%) & $139(64.1 \%)$ \\
\hline Mastectomy, N (\%) & $63(29.3 \%)$ \\
\hline Other, N (\%) & $15(6.9 \%)$ \\
\hline Patients tested for COVID-19, N (\%) & $21(9.7 \%)$ \\
\hline \multicolumn{2}{|l|}{ Complications } \\
\hline No complications, N (\%) & $199(91.7 \%)$ \\
\hline Minor complications, N (\%) & $10(4.6 \%)$ \\
\hline Major complications, N (\%) & $8(3.7 \%)$ \\
\hline
\end{tabular}

Data presented as mean \pm standard deviation or $\mathrm{n}(\%)$, unless noted otherwise.

Abbreviations: ASA = American Society of Anesthesiologists; $\mathrm{BCT}=$ breast conserving therapy; $\mathrm{BMI}=$ body mass index; COVID-19 = coronavirus disease 2019; IQR = interquartile range. 


\section{Breast Surgery Types During the Study Period}

The number of the different types of breast surgery performed from March 9 to May 17 are presented Figure 1. An overall decline occurred in the total number of surgical procedures performed that was most pronounced after week 6 of the study period. However, in the last 2 weeks (weeks 9 and 10), a slight increase occurred in the total number of breast cancer procedures performed. The number of BCT procedures had declined steady throughout, except for the slight increase in the last 2 weeks of the study period. However, the number of mastectomies and other types of breast surgery had remained stable over time. No significant differences were found in the proportion of surgical procedures performed when stratified by the study week $(P=0.173)$. 

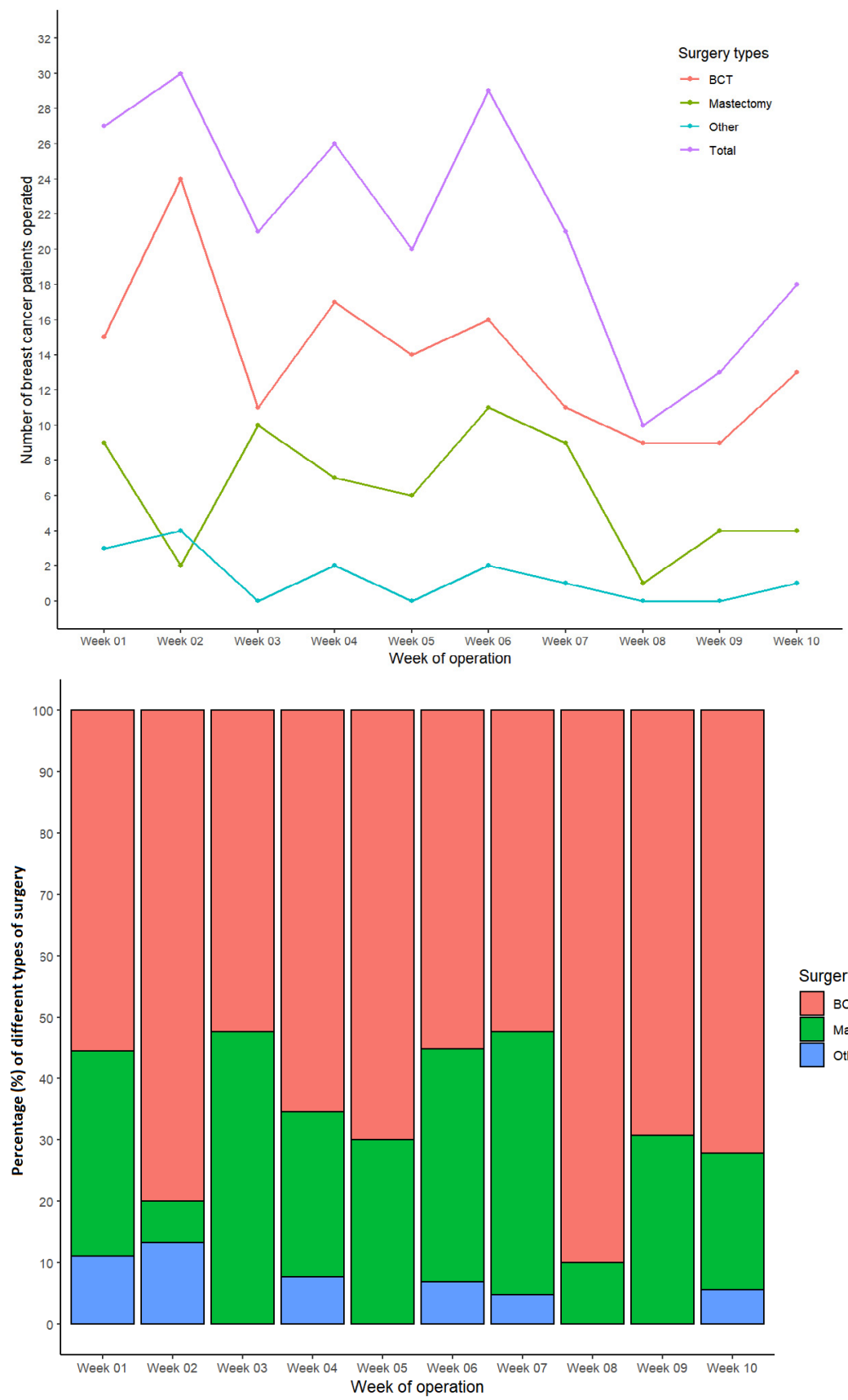

Surgery types

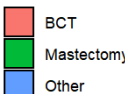

Figure 1. Number of Breast Cancer Surgeries Presented by Type of Surgical Procedure Abbreviation: $\mathrm{BCT}=$ breast-conserving therapy. 


\section{Initial Referral and $\mathrm{T}$ and $\mathrm{N}$ Stage}

The route of referral, including the number of patients referred by GPs or the national screening program, is presented in Figure 2. During the study period, an overall decrease was found in the number of patients with breast cancer requiring surgery who had originally been diagnosed through the national screening program. However, the number of patients who had been referred by their GP had declined sharply after week 6 of the study, although the number had increased again after 8 weeks.

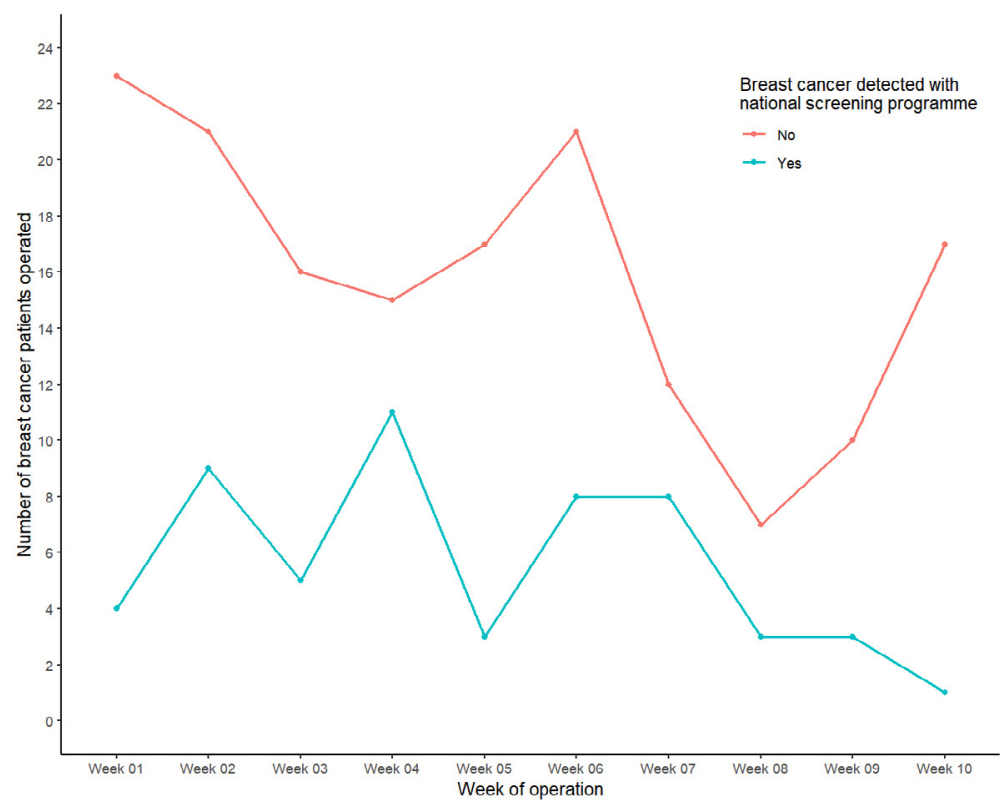

Figure 2. Number and Percentage of Patients With Breast Cancer Who Had Undergone Surgery From March 9 to May 17, 2020 With Referral From National Screening Program or General Practitioner

The number of breast cancer patients with T1 and N1 tumors had gradually declined during the study period. A decrease in the number of patients with stage N0 tumors was seen after week 6 . The other $\mathrm{T}$ and $\mathrm{N}$ stages remained stable during the study period (Figure 3). 

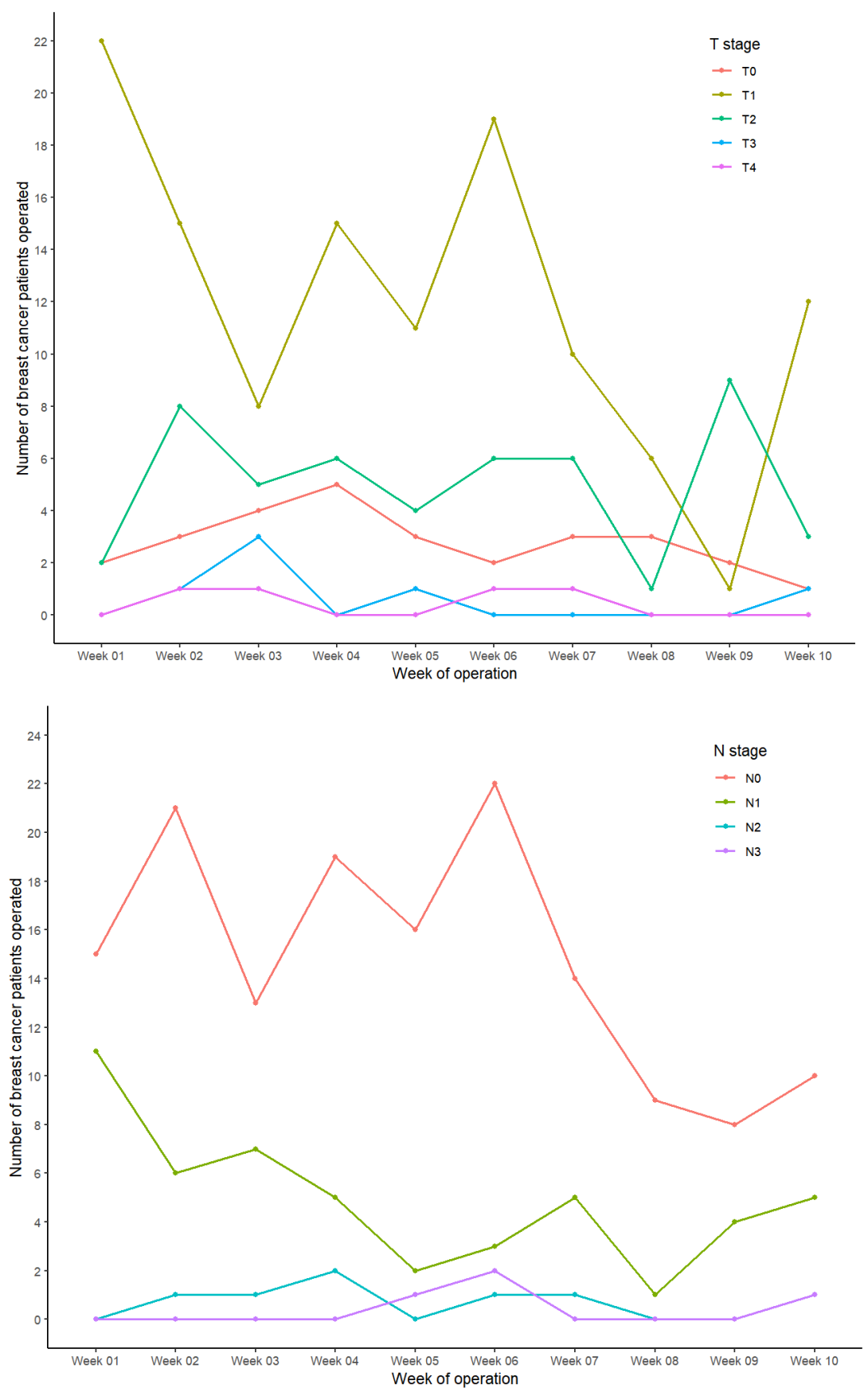

Figure 3. Number of Surgical Procedures Stratified by T and N Stage 


\section{Complications}

No increase in the number or severity of postoperative complications was found during the study period. Multivariate analysis demonstrated that only the number of comorbidities and surgery type contributed to the risk of postoperative complications in the patients requiring breast cancer surgery (Table 2). Patients undergoing mastectomy had a significantly greater risk of developing postoperative complications (OR, 3.73; 95\% CI, 1.14-12.23; P = .030) compared with patients undergoing BCT. Likewise, the number of comorbidities increased the risk of postoperative complications (OR, 1.95; 95\% CI, 1.05-3.45; P = .035). COVID-19-related symptoms and neoadjuvant therapy did not increase the risk of postoperative complications.

Table 2. Multivariate Analysis Results of Risk of Postoperative Complications

\begin{tabular}{llllll}
\hline Parameter & Estimate & OR $(\mathbf{9 5 \%} \mathbf{C I})$ & Standard error & $\mathbf{Z}$ value & P value \\
\hline Week number & -0.143 & $0.87(0.70-1.08)$ & 0.11 & -1.301 & 0.193 \\
Patient age & 0.038 & $1.04(0.99-1.09)$ & 0.026 & 1.478 & 0.140 \\
BMI & -0.033 & $0.97(0.89-1.05)$ & 0.042 & -0.801 & 0.423 \\
Number comorbidities & 0.670 & $1.95(1.05-3.65)$ & 0.318 & 2.104 & 0.035 \\
ASA classification & 0.405 & $1.50(0.53-4.26)$ & 0.533 & 0.760 & 0.448 \\
T stage & -0.190 & $0.83(0.40-1.70)$ & 0.369 & -0.516 & 0.606 \\
N stage & -0.310 & $0.73(0.26-2.07)$ & 0.529 & -0.586 & 0.558 \\
Type of surgery & & & & & \\
$\quad$ BCT & $\mathrm{NA}$ & $1.00($ reference $)$ & $\mathrm{NA}$ & $\mathrm{NA}$ & $\mathrm{NA}$ \\
$\quad \begin{array}{l}\text { Mastectomy } \\
\quad \text { Other }\end{array}$ & 1.318 & $3.73(1.14-12.23)$ & 0.605 & 2.177 & 0.030 \\
Neo-adjuvant therapy & 0.583 & $1.79(0.15-21.74)$ & 1.273 & 0.458 & 0.647 \\
COVID-19 symptoms & 0.625 & $1.87(0.50-7.02)$ & 0.675 & 0.926 & 0.355 \\
\hline
\end{tabular}

Abbreviations: ASA = American Society of Anesthesiologists; $\mathrm{BCT}=$ breast-conserving therapy; $\mathrm{BMI}=$ body mass index; $\mathrm{CI}=$ confidence interval; COVID-19 = coronavirus disease 2019; OR = odds ratio, NA = not applicable; $\mathrm{SE}=$ standard error. 


\section{Discussion}

The results from the present multicenter retrospective cohort study showed an overall decrease in the number of breast cancer surgeries performed during the COVID-19 pandemic in the Netherlands. Furthermore, the number of referrals from the national screening program and GPs had declined. Also, no increase in the number of postoperative complications occurred during the study period. Finally, the presence of COVID-19related symptoms did not increase the risk of postoperative complications.

The COVID-19 pandemic has placed an enormous strain on healthcare systems worldwide. Many measures taken by hospitals in less affected areas, such as the reallocation of hospital resources and prioritizing care, were based on the experiences from the countries highly affected by the pandemic. ${ }^{20-22}$ For surgical procedures, the guidelines advised providing only the most essential (oncologic) care to accommodate the increased demand for the care of patients with COVID-19 in hospitals. In addition, this advice was given to reduce the risk of postoperative complications in patients with COVID-19 and to reduce the risk of spreading the disease. ${ }^{23,24}$ As in other countries, in the Netherlands, the authorities recommended only performing essential surgery. ${ }^{6}$ Furthermore, the Dutch national screening program for breast cancer was temporarily halted from mid-March 2020 to mid-June 2020 to allow for reallocation of healthcare workers. ${ }^{4}$ Before the COVID-19 pandemic, $\sim 40 \%$ of new-onset breast cancer cases were detected through the national screening program. ${ }^{9,10}$ The combination of the temporary halt of national screening and the recently reported decline in new breast cancer diagnoses is worrisome. ${ }^{9,10}$

The halt of the national screening programs affected the number of surgical procedures for breast cancer. The results from the present study demonstrated a sharp decrease in the number of breast cancer surgeries. This decrease occurred because surgical resection with or without radiotherapy and/or systemic treatment is the treatment of choice for patients with new-onset breast cancer. ${ }^{11-13}$ Our results have confirmed previous findings that, nationwide, fewer breast cancer cases have been diagnosed during the COVID-19 pandemic. ${ }^{4}$ Furthermore, this decrease was especially prominent 6 weeks after the temporary closure of the national screening program, equal to week 7 in our study period. In the Netherlands, treatment (neoadjuvant therapy, radiotherapy, and/or surgery) is normally required within 6 weeks after the initial cancer diagnosis. ${ }^{25}$ Therefore, a decline in the number of surgical procedures could be expected 6 weeks after halting national screening. Moreover, the present study found that, not only had a decrease occurred in those referred through the national screening program, but also a decreased had occurred in those referred by GPs. However, at the beginning of the pandemic, the Dutch government had discouraged patients from visiting their GP unless absolutely necessary. Although the number of patients referred by GPs had increased steadily in the last weeks of the study, the number of patients referred by national screening had decreased further. The increase of GP referrals might have resulted from awareness campaigns by the Dutch government later in the study period, which encouraged patients with symptoms to visit their GP. ${ }^{26}$ 
The present study demonstrated a decline in stage T1-T2 and N0 tumors, which can be attributed to the temporary closure of the Dutch national screening program. This was not surprising because most screening-detected breast cancer cases will be diagnosed at an early stage. ${ }^{27}$ However, we do not believe that the temporary halting of the breast cancer screening program will have a significant effect on long-term outcomes because most breast cancer discovered by the screening program will be early-stage breast cancer that will develop slowly. Thus, we do not believe an increase will occur in the number of higher stage breast cancer cases because the breast cancer screening program was stopped for only 4 months. Our findings have confirmed previous findings from the Netherlands Comprehensive Cancer Organisation, which found fewer breast cancer diagnoses nationwide during the COVID-19 pandemic. $^{4}$ The number of cases with higher $\mathrm{T}$ and $\mathrm{N}$ stages appeared to remain relatively stable over time. The decrease in stage T1-T2 and N0 tumors also explains the decrease in BCT procedures performed, because BCT is preferred over mastectomy for these tumor stages. ${ }^{11-13}$ Despite the relatively small risk of postoperative complications from breast cancer surgery, many studies have recommended postponing or not performing these procedures during the pandemic. ${ }^{23,24,28,29}$ However, we found no increase in postoperative complications in the patients undergoing breast cancer surgery during the COVID-19 pandemic in our study. The multivariate analysis results showed that patients undergoing mastectomy and patients with multiple comorbidities had an increased risk of developing postoperative complications. However, these findings are in line with those from previous studies reporting a significantly greater complication rate for patients undergoing mastectomy and patients with more comorbidities. ${ }^{28,29}$

The present study had some limitations. First, the number of included patients was relatively small. Therefore, the number of stage T3-T4 tumors was low, making the recognition of patterns for this patient group more difficult. The relative low number of stage $\mathrm{N} 2-\mathrm{N} 3$ tumors posed similar challenges in pattern recognition over time. However, the findings from our study did show a clear decreasing pattern in the number of lower stage tumors during the study period. Second, only 21 patients were tested for COVID-19, and the tests results for all 21 were negative. No change occurred in the incidence of postoperative complications. However, more research is necessary to determine the direct risk of COVID-19 positivity compared with COVID-19 negativity on the development of postoperative complications for patients undergoing breast cancer surgery. Therefore, it was not possible to determine the direct risk of the presence of COVID-19 on the development of postoperative complications in patients with breast cancer. Third, significant fluctuations were found in the weekly number of patients undergoing surgical procedures, which most likely resulted from the relatively small number of patients. Finally, the present study had focused on surgical care, although nonsurgical care (ie, radiotherapy, chemotherapy, antihormonal therapy) has also likely to have been affected by the COVID-19 pandemic. ${ }^{30,31}$ Because nonsurgical care was not addressed in our study, we could not report the effects of the COVID-19 pandemic on breast cancer treatment in all its facets. 


\section{Conclusions}

Considering these limitations, the present study has shown that the effect of the COVID-19 pandemic has dramatically decreased the number of breast cancer surgeries performed. This decrease resulted not only from the temporary closure of the national screening program but also from fewer referrals from GPs. We found no change in the number of postoperative complications. However, more research is necessary to determine the direct risk of developing postoperative complications for patients with COVID-19 undergoing breast cancer compared with the risk for patients without COVID-19. Furthermore, provided that the necessary precautions have been taken to avoid the spread of COVID-19, patients should be encouraged to visit their GP, and one should strive to restart national screening programs. However, in the event of a second wave, we would recommend temporary closure of the national screening program but the continuation of surgical procedures for patients referred by GPs 


\section{References}

1. Li H, Liu S-M, Yu X-H, Tang S-L, Tang C-K. Coronavirus disease 2019 (COVID-19): current status and future perspectives. Int J Antimicrob Agents. 2020;55(5):105951. doi:10.1016/j.ijantimicag.2020.105951

2. Corman VM, Landt O, Kaiser M, et al. Detection of 2019 novel coronavirus (2019-nCoV) by real-time RT-PCR. Eurosurveillance. 2020;25(3). doi:10.2807/1560-7917.ES.2020.25.3.2000045

3. Johns Hopkins CSSE. Coronavirus COVID-19 Global Cases by the Center for Systems Science and Engineering (CSSE) at Johns Hopkins University (JHU. https://gisanddata.maps.arcgis.com/apps/ opsdashboard/index.html\#/bda7594740fd40299423467b48e9ecf6.

4. Dinmohamed AG, Visser O, Verhoeven RHA, et al. Fewer cancer diagnoses during the COVID-19 epidemic in the Netherlands. Lancet Oncol. April 2020. doi:10.1016/S1470-2045(20)30265-5

5. Burki TK. Cancer guidelines during the COVID-19 pandemic. Lancet Oncol. 2020;21(5):629-630 doi:10.1016/S1470-2045(20)30217-5

6. NVVH. Handvat voor chirurgische ingrepen tijdens Corona-crisis. 2020.

7. Nederland I kankercentrum. COVID 19 en borstkanker.

8. Bray F, Ferlay J, Soerjomataram I, Siegel RL, Torre LA, Jemal A. Global cancer statistics 2018: GLOBOCAN estimates of incidence and mortality worldwide for 36 cancers in 185 countries. CA Cancer J Clin. 2018;68(6):394-424. doi:10.3322/caac.21492

9. Vondeling GT, Menezes GL, Dvortsin EP, et al. Burden of early, advanced and metastatic breast cancer in The Netherlands. BMC Cancer. 2018;18(1):262-263. doi:10.1186/s12885-018-4158-3 [doi]

10. Koleva-Kolarova RG, Daszczuk AM, de Jonge C, et al. A modelling study to evaluate the costs and effects of lowering the starting age of population breast cancer screening. Maturitas. 2018;109:81-88. doi:S0378-5122(17)30543-1 [pii]

11. Agarwal S, Pappas L, Neumayer L, Kokeny K, Agarwal J. Effect of breast conservation therapy vs mastectomy on disease-specific survival for early-stage breast cancer. JAMA Surg. 2014;149(3):267-274 doi:10.1001/jamasurg.2013.3049 [doi]

12. Hartmann-Johnsen OJ, Karesen R, Schlichting E, Nygard JF. Survival is Better After Breast Conserving Therapy than Mastectomy for Early Stage Breast Cancer: A Registry-Based Follow-up Study of Norwegian Women Primary Operated Between 1998 and 2008. Ann Surg Oncol. 2015;22(12):38363845. doi:10.1245/s10434-015-4441-3 [doi]

13. van Maaren MC, de Munck L, de Bock GH, et al. 10 year survival after breast-conserving surgery plus radiotherapy compared with mastectomy in early breast cancer in the Netherlands: a population-based study. The LancetOncology. 2016;17(8):1158-1170. doi:S1470-2045(16)30067-5 [pii]

14. Owens WD. American Society of Anesthesiologists Physical Status Classification System in not a risk classification system. Anesthesiology. 2001;94(2):378. doi:10.1097/00000542-200102000-00042

15. Dindo D, Demartines N, Clavien PA. Classification of surgical complications: a new proposal with evaluation in a cohort of 6336 patients and results of a survey. Ann Surg. 2004;240(2):205-213. doi:00000658-200408000-00003 [pii]

16. Rao AA, Feneis J, Lalonde C, Ojeda-Fournier H. A Pictorial Review of Changes in the BI-RADS Fifth Edition. Radiographics. 2016;36(3):623-639. doi:10.1148/rg.2016150178 [doi]

17. Tan BY, Acs G, Apple SK, et al. Phyllodes tumours of the breast: a consensus review. Histopathology. 2016;68(1):5-21. doi:10.1111/his.12876 
18. Beaulieu-Jones BK, Lavage DR, Snyder JW, Moore JH, Pendergrass SA, Bauer CR. Characterizing and Managing Missing Structured Data in Electronic Health Records: Data Analysis. JMIR Med informatics. 2018;6(1):e11. doi:10.2196/medinform.8960 [doi]

19. Zhang Z. Multiple imputation with multivariate imputation by chained equation (MICE) package. Ann Transl Med. 2016;4(2):30-5839.2015.12.63. doi:10.3978/j.issn.2305-5839.2015.12.63 [doi]

20. Ravikumar N, Nallasamy K, Bansal A, et al. Novel Coronavirus 2019 (2019-nCoV) Infection: Part I Preparedness and Management in the Pediatric Intensive Care Unit in Resource-limited Settings. Indian Pediatr. March 2020.

21. Guest JL, Del Rio C, Sanchez T. The 3 Steps Needed to End the COVID-19 Pandemic: Bold Public Health Leadership, Rapid Innovations, and Courageous Political Will. JMIR public Heal Surveill. April 2020. doi:10.2196/19043

22. Zangrillo A, Beretta L, Silvani $P$, et al. Fast reshaping of intensive care unit facilities in a large metropolitan hospital in Milan, Italy: facing the COVID-19 pandemic emergency. Crit Care Resusc. April 2020.

23. Al-Jabir A, Kerwan A, Nicola M, et al. Impact of the Coronavirus (COVID-19) pandemic on surgical practice - Part 1. Int J Surg. 2020;79:168-179. doi:10.1016/j.ijsu.2020.05.022

24. Al-Jabir A, Kerwan A, Nicola M, et al. Impact of the Coronavirus (COVID-19) pandemic on surgical practice - Part 2 (surgical prioritisation). Int J Surg. 2020;79:233-248. doi:10.1016/j.ijsu.2020.05.002

25. SONCOS. Dutch guidelines stating that all patients must have had the first treatment within 6 weeks after cancer diagnosis. https://www.nvog.nl/wp-content/uploads/2018/02/SONCOS-normeringsrapportversie-5-2017.pdf.

26. Dutch Ministry. Campaign to encourage patients to visit the general practioner during COVID-19 in order to keep up with cancer diagnoses [reference in Dutch]. https://www.rijksoverheid.nl/documenten/ mediateksten/2020/04/17/letterlijke-tekst-persconferentie-na-ministerraad-17-april-2020.

27. Elferink MAG, Toes-Zoutendijk E, Vink GR, et al. [National population screening for colorectal carcinoma in the Netherlands: results of the first years since the implementation in 2014]. Ned Tijdschr Geneeskd. 162:D2283.

28. El-Tamer MB, Ward BM, Schifftner T, Neumayer L, Khuri S, Henderson W. Morbidity and Mortality Following Breast Cancer Surgery in Women. Ann Surg. 2007;245(5):665-671. doi:10.1097/01. sla.0000245833.48399.9a

29. ten Wolde B, Kuiper M, de Wilt JHW, Strobbe LJA. Postoperative Complications After Breast Cancer Surgery are Not Related to Age. Ann Surg Oncol. 2017;24(7):1861-1867. doi:10.1245/s10434-0165726-x

30. van Maaren MC, Strobbe LJA, Koppert LB, Poortmans PMP, Siesling S. Nationwide populationbased study of trends and regional variation in breast-conserving treatment for breast cancer. Br J Surg. 2018;105(13):1768-1777. doi:10.1002/bjs.10951

31. Kilsdonk MJ, van Dijk BA, Otter R, van Harten WH, Siesling S. Regional variation in breast cancer treatment in the Netherlands and the role of external peer review: a cohort study comprising 63,516 women. BMC Cancer. 2014;14(1):596. doi:10.1186/1471-2407-14-596 


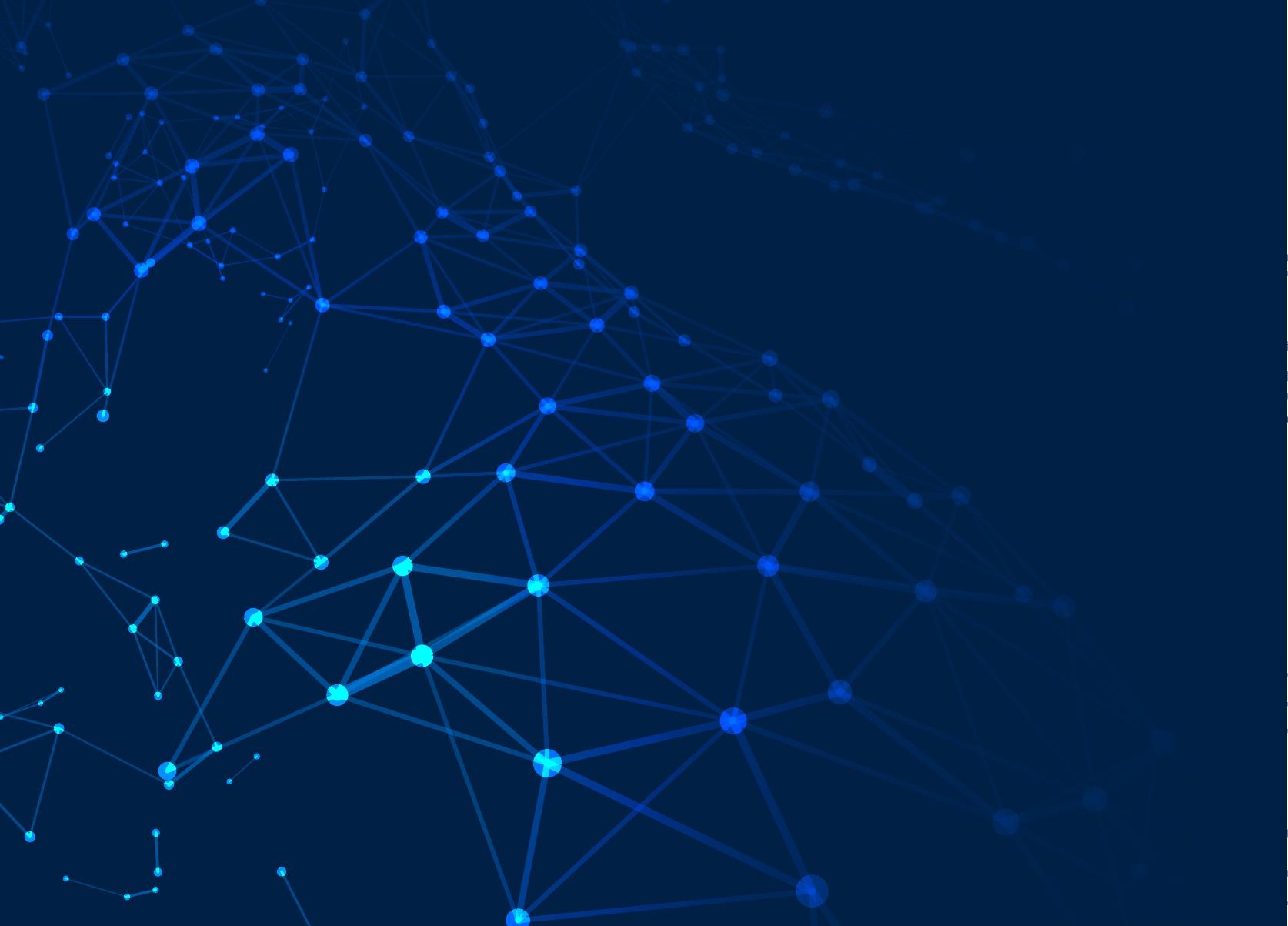





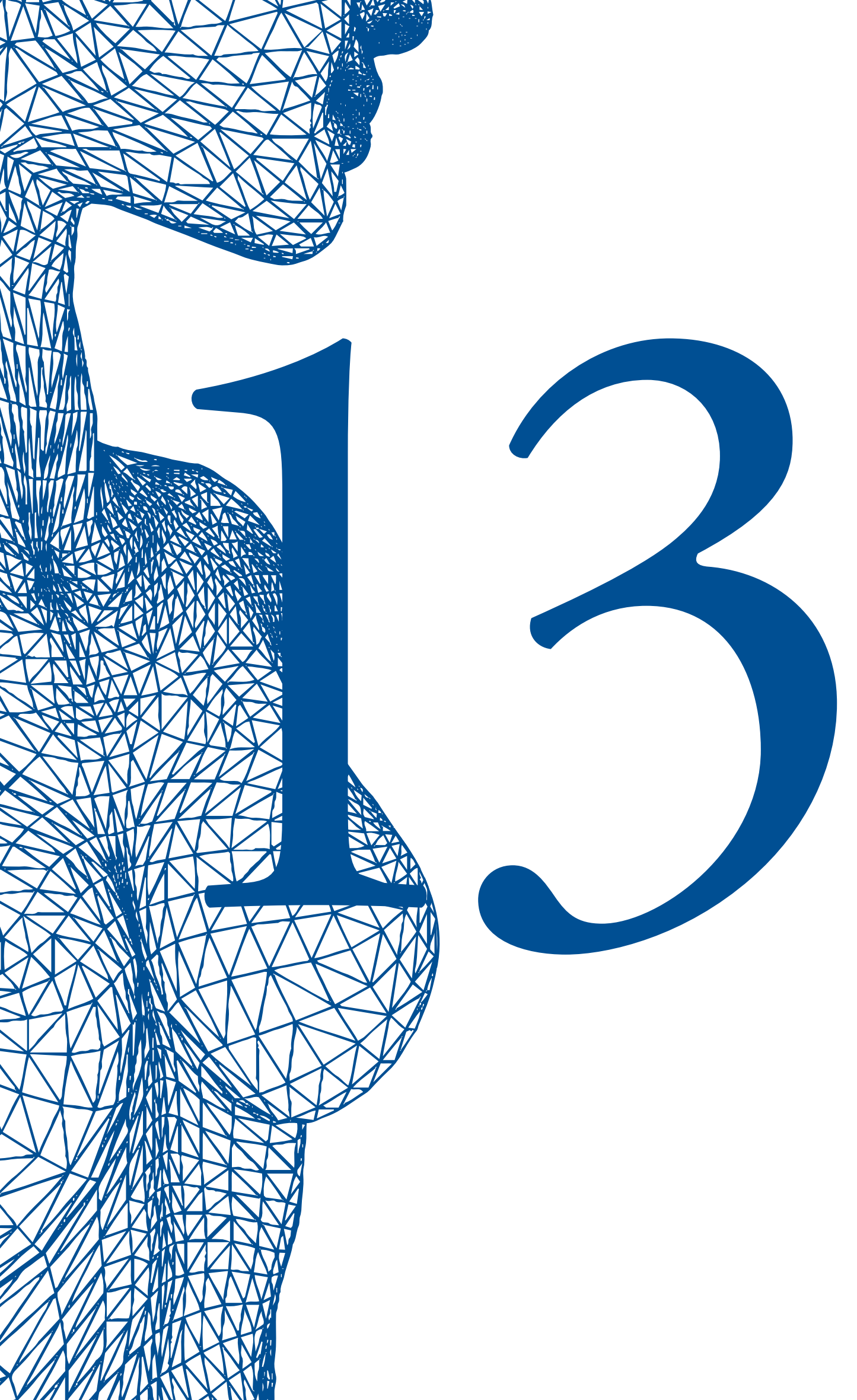


Chapter 13

General discussion and future perspectives 


\section{Ductoscopy}

Duct excision surgery is currently used to rule out malignancy and treat patients with pathological nipple discharge (PND) without radiological signs of malignancy. ${ }^{1-3}$ Duct excision surgery is performed under general anesthesia and may result in reduced sensitivity of the nipple and breastfeeding difficulties in fertile women. ${ }^{4-8}$ However, only around $5 \%$ of these patients actually have a malignancy. ${ }^{3,9}$ Further diagnostics are warranted to improve patient selection for duct excision surgery to limit unnecessary invasive procedures and its associated potential morbidities. Currently, there is no consensus on what the best diagnostic strategy is for patients with PND.

White light ductoscopy has shown to be able to detect (pre)cancerous lesions in patients with PND that were missed during mammography and ultrasound. ${ }^{10}$ Yet, pooled sensitivity of ductoscopy is lower than that of MRI (44\% vs 76\%) however specificity of ductoscopy showed to be higher (98\% vs. $84 \%) .{ }^{11}$ Autofluorescence ductoscopy has shown to be feasible and effective in increasing the detection rate of (pre)cancerous lesions during ductoscopy. ${ }^{12-14}$ However, there are currently no ready to use autofluorescence devices available that can be connected to ductoscopy devices. Alternatively, narrow band imaging (NBI) is an enhanced imaging technique that also allows visualization of (pre)cancerous lesions of epithelial origin that are missed during conventional scopic procedures by.${ }^{15-18}$ Devices with NBI capabilities are widely used and can be easily connected to ductoscopy systems. ${ }^{19}$ For this reason NBI has a lot of potential to increase the sensitivity of ductoscopy for the detection of breast cancer.

Current biopsy tools used during ductoscopy procedures (such as the endobasket) have their back draws. They do not always succeed in complete removal of lesions causing PND and taking a biopsy of small lesions is difficult or even impossible. ${ }^{10,20}$ Therefor new biopsy devices specially designed for micro endoscopic use are an essential next step in enhancing the diagnostic and therapeutic effectivity of ductoscopy, allowing further reduction of duct excision surgery. Currently the Technical University Delft is developing such next generation biopsy devices in close collaboration with the department of surgical oncology of the University Medical Center Utrecht (UMCU). ${ }^{19}$ Also the addition of intraductal laser ablation of remnants of intraductal lesions after incomplete biopsy might enhance the therapeutic effectivity of ductoscopy in PND patients even further in near future. A previous ex-vivo study has already shown that intraductal laser ablation is feasible. ${ }^{21}$

Currently, the UMCU is the only center performing ductoscopy procedures in the Netherlands. This is mainly because ductoscopy is not reimbursed by healthcare insurance companies yet. In 2020, the procedure to include ductoscopy in the basic health insurance package has been started by ZIN (Zorg Instituut Nederland). Since ductoscopy is included in the "Dutch guideline breast cancer" as treatment option in PND, we expect 
that when reimbursement is secured more hospitals will incorporate ductoscopy in the work-up in PND patients. This has the potential of significantly reducing the costs for the diagnosis and treatment of PND in the Netherlands. ${ }^{22}$

Successful reimbursement will also facilitate initiation of larger multicenter studies in the Netherlands, to evaluate the effectiveness of NBI and laser ablation for example, in the treatment of PND). ${ }^{19}$

Also, the possible role of ductoscopy in the detection of breast cancer precursor lesions in woman at high risk for breast cancer (i.e. BRCA mutation carriers) needs to be investigated when the sensitivity of ductoscopy is optimized. Addition of ductoscopy in the screening of this group of women with a lifetime breast cancer risk of $60-80 \%$ might make the screening process more safe and this in turn might lead to postponement of prophylactic mastectomy in some cases (till after pregnancy for example). ${ }^{23,24}$

\section{Robotic nipple sparing mastectomy}

Robotic nipple sparing mastectomy (RNSM) is a promising new minimally invasive technique that may replace the current invasive surgical mastectomy. ${ }^{25-29}$ This thesis explored the safety of RNSM compared with traditional mastectomy, both in the preventive as therapeutic setting. The long-term oncological safety of RNSM has yet to be established. However, the scopic nature of RNSM allows enhanced imaging such as NBI. This could expand the use of Robotic surgery to breast conserving surgery. Currently, worldwide efforts are being made, in cooperation with Intuitive ${ }^{\mathrm{TM}}$, in expanding the intended use of the Da Vinci ${ }^{\circledR}$ system to breast surgery. CE marking would facilitate studies that could explore the benefits of minimally invasive breast cancer surgery. This has the potential to significantly improve cosmetic outcomes.

\section{Socioeconomic status and breast cancer treatment}

The current thesis shows that socioeconomic status (SES) influences treatment in patients with breast cancer in the Netherlands. Breast cancer patients with a lower SES tend to receive different treatment compared with patients with a higher SES. This is an unpleasant finding, since Dutch laws and values strive for (healthcare) equality for all citizens. Prospective studies are warranted to determine whether these differences are subconsciously initiated by the treating physician because these patients might be less articulate when it comes to choice of treatment. If so, it is possible that physicians need to be trained on how to provide optimal decisional information to the lower strata of the SES. However, patients with lower SES statistically also have more health related problems and this might also be a reason for different treatment choices. 


\section{Breast cancer surgery during COVID-19}

Coronavirus disease 2019 (COVID-19) has put an enormous strain on healthcare systems worldwide. In the Netherlands this has led to a national temporary stoppage of breast cancer screening between $16^{\text {th }}$ of March 2020 and $1^{\text {st }}$ of July 2020. Consequently, the number of lower staged tumors being diagnosed and operated on has declined. ${ }^{30,31}$ In the midst of the second wave there needs to be reallocations of healthcare resources once again. Based on the current data, halting the national screening program for breast cancer is relatively safe. This can be declared by the fact that breast cancer screening mostly detects in situ carcinoma's or small low staged cancers and it takes a relatively long period of time for these lesions to develop in higher (more difficult to treat) stage cancers with worse outcome. ${ }^{32}$ This decreases demand of hospital resources needed for breast cancer treatment while at the same time reducing the movement of people and thereby reducing the spread of COVID-19. However, long term consequences of temporary cessation of the national screening program for breast cancer are yet to be determined. 


\section{References}

1. Bahl M, Baker JA, Greenup RA, Ghate S V. Diagnostic Value of Ultrasound in Female Patients With Nipple Discharge. AJRAmerican J Roentgenol. 2015;205(1):203-208. doi:10.2214/AJR.14.13354 [doi]

2. Sanders LM, Daigle M. The Rightful Role of MRI after Negative Conventional Imaging in the Management of Bloody Nipple Discharge. Breast J. 2016;22(2):209-212. doi:10.1111/tbj.12551 [doi]

3. Alcock C, Layer GT. Predicting occult malignancy in nipple discharge. ANZ J Surg. 2010;80(9):646-649. doi:10.1111/j.1445-2197.2010.05270.x [doi]

4. Dixon JM, Mansel RE. ABC of breast diseases. Symptoms assessment and guidelines for referral. BMJ. 1994;309(6956):722-726.

5. Seltzer MH. Breast complaints, biopsies, and cancer correlated with age in 10,000 consecutive new surgical referrals. Breast J. 2004;10(2):111-117. doi:21284 [pii]

6. Santen RJ, Mansel R. Benign breast disorders. N Engl J Med. 2005;353(3):275-285. doi:353/3/275 [pii]

7. King TA, Carter KM, Bolton JS, Fuhrman GM. A simple approach to nipple discharge. Am Surg. 2000;66(10):960-966.

8. Sarakbi W Al, Worku D, Escobar PF, Mokbel K. Breast papillomas: current management with a focus on a new diagnostic and therapeutic modality. Int Semin Surg Oncol. 2006;3:1. doi:1477-7800-3-1 [pii]

9. Albrecht $\mathrm{C}$, Thele F, Grunwald S, et al. Nipple discharge: role of ductoscopy in comparison with standard diagnostic tests. Onkologie. 2013;36(1-2):12-16. doi:10.1159/000346639 [doi]

10. Filipe MD, Waaijer L, van der Pol CC, van Diest P, Witkamp AJ. Interventional ductoscopy as an alternative for major duct excision or microdochectomy in women suffering pathological nipple discharge: a single centre experience. Clin Breast Cancer. Published online January 27, 2020. doi:10.1016/j. clbc.2019.12.008

11. Filipe MD, Patuleia SIS, de Jong VMT, Vriens MR, van Diest PJ, Witkamp AJ. A network meta-analysis for the diagnostic approach to pathological nipple discharge. Clin Breast Cancer. Published online June 2020. doi:10.1016/j.clbc.2020.05.015

12. Waaijer L, Filipe MD, Simons J, et al. Detection of breast cancer precursor lesions by autofluorescence ductoscopy. Breast Cancer. Published online July 28, 2020. doi:10.1007/s12282-020-01136-6

13. Douplik A, Leong WL, Easson AM, Done S, Netchev G, Wilson BC. Feasibility study of autofluorescence mammary ductoscopy. J Biomed Opt. 2009;14(4):44036. doi:10.1117/1.3210773 [doi]

14. Jacobs VR, Paepke S, Schaaf H, Weber BC, Kiechle-Bahat M. Autofluorescence ductoscopy: a new imaging technique for intraductal breast endoscopy. Clin Breast Cancer. 2007;7(8):619-623. doi:S1526-8209(11)70750-9 [pii]

15. Singh R, Mei SC, Sethi S. Advanced endoscopic imaging in Barrett's oesophagus: a review on current practice. World J Gastroenterol. 2011;17(38):4271-4276. doi:10.3748/wjg.v17.i38.4271 [doi]

16. Efthymiou M, Taylor AC, Kamm MA. Cancer surveillance strategies in ulcerative colitis: the need for modernization. Inflamm Bowel Dis. 2011;17(8):1800-1813. doi:10.1002/ibd.21540 [doi]

17. Tanaka S, Sano Y. Aim to unify the narrow band imaging (NBI) magnifying classification for colorectal tumors: current status in Japan from a summary of the consensus symposium in the 79th Annual Meeting of the Japan Gastroenterological Endoscopy Society. Dig Endosc. 2011;23 Suppl 1:131-139. doi:10.1111/j.1443-1661.2011.01106.x [doi] 
18. Jichlinski P, Lovisa B. High magnification cystoscopy in the primary diagnosis of bladder tumors. Curr Opin Urol. 2011;21(5):398-402. doi:10.1097/MOU.0b013e32834956ad [doi]

19. Filipe, Mando D.; Sakes, Aimée; Rem, Alexander I.; Breedveld, Paul B.; Euwe, Froukje E.; Vriens, Menno R.; van Diest, Paul J; Witkamp AJ. Narrow band imaging, intraductal biopsy and intraductal laser ablation during ductoscopy: a study protocol.

20. Waaijer L, van Diest PJ, Verkooijen HM, et al. Interventional ductoscopy in patients with pathological nipple discharge. Br J Surg. 2015;102(13):1639-1648. doi:10.1002/bjs.9950 [doi]

21. de Boorder T, Waaijer L, van Diest PJ, Witkamp AJ. Ex vivo feasibility study of endoscopic intraductal laser ablation of the breast. Lasers Surg Med. 2018;50(2):137-142. doi:10.1002/1sm.22745

22. Filipe, M.D.; Patuleia, S.I.S.; Vriens, M.R.; van Diest, P.J.; Witkamp AJ. Meta-analysis and costeffectiveness of ductoscopy, duct excision surgery and MRI for the diagnosis and treatment of patients with pathological nipple discharge.

23. Ford D, Easton DF, Stratton M, et al. Genetic heterogeneity and penetrance analysis of the BRCA1 and BRCA2 genes in breast cancer families. The Breast Cancer Linkage Consortium. Am J Hum Genet. 1998;62(3):676-689. doi:S0002-9297(07)63848-8 [pii]

24. Tung N, Battelli C, Allen B, et al. Frequency of mutations in individuals with breast cancer referred for BRCA1 and BRCA2 testing using next-generation sequencing with a 25-gene panel. Cancer. 2015;121(1):25-33. doi:10.1002/cncr.29010 [doi]

25. Toesca A, Peradze N, Manconi A, et al. Robotic nipple-sparing mastectomy for the treatment of breast cancer: Feasibility and safety study. Breast. 2017;31:51-56. doi:S0960-9776(16)30191-6 [pii]

26. Park HS, Lee J, Lee DW, et al. Robot-assisted Nipple-sparing Mastectomy with Immediate Breast Reconstruction: An Initial Experience. Sci Rep. 2019;9(1):15669. https://pubmed.ncbi.nlm.nih. gov/31666551

27. Lai H-W, Chen S-T, Lin S-L, et al. Robotic Nipple-Sparing Mastectomy and Immediate Breast Reconstruction with Gel Implant: Technique, Preliminary Results and Patient-Reported Cosmetic Outcome. Ann Surg Oncol. 2019;26(1):42-52. https://pubmed.ncbi.nlm.nih.gov/30109537

28. Kim H, Park S-J, Woo K-J, Bang SI. Comparative Study of Nipple-Areola Complex Position and Patient Satisfaction After Unilateral Mastectomy and Immediate Expander-Implant Reconstruction NippleSparing Mastectomy Versus Skin-Sparing Mastectomy. Aesthetic Plast Surg. 2019;43(2):313-327. https:// pubmed.ncbi.nlm.nih.gov/30746565

29. Pek W-S, Tan B-K, Ru Ng YY, et al. Immediate breast reconstruction following nipple-sparing mastectomy in an Asian population: Aesthetic outcomes and mitigating nipple-areolar complex necrosis. Arch Plast Surg. 2018;45(3):229-238. https://pubmed.ncbi.nlm.nih.gov/29788682

30. Dinmohamed AG, Visser O, Verhoeven RHA, et al. Fewer cancer diagnoses during the COVID-19 epidemic in the Netherlands. Lancet Oncol. Published online April 2020. doi:10.1016/S14702045(20)30265-5

31. Filipe MD, van Deukeren D, Kip M, et al. Effect of the COVID-19 Pandemic on Surgical Breast Cancer Care in the Netherlands: A Multicenter Retrospective Cohort Study. Clin Breast Cancer. Published online 2020. doi:10.1016/j.clbc.2020.08.002

32. Evans AJ, Wilson ARM, Pinder SE, Ellis IO, Sibbering DM, Yeoman LJ. Ductal carcinoma in situ: Imaging, pathology and treatment. IMAGING. 1994;6(3):171-184. 
General discussions, future perspectives and conclusions 
Chapter 14

Summary 


\section{Part I. Intraductal diagnosis of breast pathology}

Ductoscopy is a minimally invasive endoscopic technique that allows real-time visualization of the milk duct of the breast. Ductoscopy is mostly used for the diagnosis and treatment in patients with pathological nipple discharge (PND). More recently, magnetic resonance imaging (MRI) is also being used for the detection of breast cancer in patients with PND. Chapter 2 compares the different diagnostic tools currently available (mammography, ultrasound, MRI, cytology and ductoscopy) in a systematic review and network metaanalysis. Thirty-six studies with a pooled sample size of 3764 women with PND underwent ultrasound, mammogram, MRI, cytology, and/or ductoscopy. Pooled sensitivity for the detection of malignancy was $50 \%, 22 \%, 83 \%, 38 \%$ and $58 \%$ respectively. Pooled specificity was $69 \%, 93 \%, 76 \%, 90 \%$ and $92 \%$ respectively for ultrasound, mammography, MRI, cytology and ductoscopy. Additionally, subgroup meta-analysis comparing ductoscopy with MRI in patients with PND and a negative mammography and ultrasound revealed that the sensitivity of MRI was higher (but not significantly, 44\% vs 76\%), however ductoscopy showed a significantly higher specificity ( $98 \%$ vs $84 \%)$. Ductoscopy therefor outperforms all other diagnostic methods due to its superior specificity in combination with the low incidence of malignancy in this patient group of $5 \%$.

The addition of a biopsy device (basket shaped metal wire) to ductoscopy makes interventions (removal of intraductal lesions) possible. This interventional ductoscopy has is a promising addition to ductoscopy to enhance diagnostic possibilities by having pathological confirmation of the cause of PND, which are mostly papilloma's and duct ectasia. Chapter 3 describes the findings of a retrospective analysis in which 215 consecutive patients were referred for ductoscopy because of PND without radiological suspicion for malignancy. Eventually 151 successful ductoscopy procedures where performed. Five out seven patients who turned out to have a malignancy that was missed during conventional imaging showed suspicious lesions during ductoscopy. In 141 out 144 patients who did not have malignancy, ductoscopy did also not show suspicious lesions. These findings, combined with the disappearance of PND in almost half of patients after ductoscopy, resulted in a reduction of duct excision surgery; only $28 \%$ of patients with PND were operated

In Chapter 4 we describe a method to increase the sensitivity of ductoscopy for the detection of (pre)malignancy. Autofluorescence has been used in multiple settings to improve the detection of cancer precursor lesions of epithelial origin. This chapter describes the feasibility and utility of autofluorescence ductoscopy in patients undergoing therapeutic and preventive mastectomy. Our study showed that that autofluorescence ductoscopy was able to detect (pre)cancerous lesions that were not detected during conventional white light ductoscopy. 
Chapter $\mathbf{5}$ is a study protocol that addresses the remaining challenges of ductoscopy, i.e relative low sensitivity and limited posibiliies to removal of small intraductal and epithelial lesions. This chapter describes a study protocol in two different patient populations: patients with PND without radiological suspicion for malignancy and patients undergoing (both prophylactic and therapeutic) breast surgery. The first group will be offered common (white light) ductoscopy followed by narrow band imaging (NBI) ductoscopy. Identified (suspicious) intraductal lesions will be removed using an intraductal biopsy device. If the extraction is incomplete, intraductal laser ablation will remove the remaining of the lesion. In the breast surgery group, patients will be offered white light ductoscopy, followed by NBI ductoscopy directly prior to their mastectomy. If a (suspicious) lesion is seen, intraductal biopsy will be performed and the remaining of the lesion will be treated with laser ablation. The different diagnostic tools in both patient groups will be evaluated taking the pathological analysis as a reference. Additionally, patients undergoing intraductal laser ablation will undergo a follow-up period of 1 year to determine whether PND has stopped. 


\section{Part II: Minimally invasive treatment of diseases of the breast}

Chapter $\mathbf{6}$ describes the cost-effectiveness of ductoscopy for the diagnosis and treatment of patients with PND without radiological signs of malignancy. The total costs of ductoscopy, duct excision surgery and MRI were respectively $€ 1,401.33$, €6,494.27 and $€ 822.13$. From the systematic research and meta-analysis, we found that ductoscopy, compared with MRI, had a lower sensitivity $(44 \%$ vs $76 \%)$ but a significantly higher specificity ( $84 \%$ vs $98 \%$ ) and diagnostic accuracy (95\% vs $83 \%$ ) for the detection of malignancy. Consequently, we constructed a model in which patients with positive ductoscopy or MRI underwent duct excision surgery for definitive diagnosis. Since an MRI was more likely to be positive (because of lower specificity), more patients would undergo surgery. Consequently, on average, a patient undergoing a diagnostic pathway with MRI would cost $€ 2,070.27$ while a patient undergoing ductoscopy would cost $€ 1,670.97$. Therefore, ductoscopy seems less costly (€399,30 less on average) and more effective (higher diagnostic accuracy) compared with MRI. Additionally, this chapter compares the cost-effectiveness of duct excision surgery to ductoscopy for the treatment of PND. This study showed that PND stopped in $51.2 \%$ and $100 \%$ of patients undergoing ductoscopy or duct excision surgery respectively. On average, since $48.3 \%$ of patients require duct excision surgery after ductoscopy, ductoscopy costs $€ 3,208.89$ while the costs of surgery are $€ 6,494.27$. This means that ductoscopy is less effective but also considerably less costly than duct excision surgery and ductoscopy can prevent surgery in almost half of patients

Patient-reported outcomes of patients undergoing ductoscopy due to PND without radiological sign of malignancy were described in Chapter 7. This chapter analyzed quality of life in 50 consecutive patients using questionnaires (Breast-Q, EQ-5D-5L and SF-36) on the day of ductoscopy, and after two weeks, three and six months. The Breast-Q and EQ-5D-5L showed no significant changes in quality of life overtime after adjusting for PND and or post ductoscopy, and outcomes of diagnosis. In the SF-36, only the vitality domain showed a slight, but significant, decrease over time. This study showed the questionnaires were valid for patients undergoing ductoscopy but more research needs to address the symptom specific issues of patients with PND.

Ductoscopy procedures have been performed in the Netherlands for a decade now in patients with PND without radiological signs of malignancy. Chapter 8 describes what developments have been made, in and outside the Netherlands, and which challenges lie ahead.

Robotic nipple sparing mastectomy (RNSM) is a promising new technique that has the potential to reduce the morbidity of patients requiring mastectomy while at the same time enhance cosmetic outcome. In Chapter 9, we performed a systematic review and 
meta-analysis describing the complication rates in patients undergoing mastectomy (both preventive and therapeutic) with immediate breast reconstruction. Forty-nine studies with 13,886 cases of (R)NSM were included in this study and RNSM had lower (but not significant) complication rate compared to traditional mastectomy (3.9\% vs. $7.0 \%$, $\mathrm{p}=0.070)$. Therefore, the less invasive RNSM seems to be an adequate alternative to traditional mastectomy. However, more research is required to determine oncological safety and cosmetic outcome of this new technique. 


\section{Part III. Influence of socioeconomic status and COVID-19 on breast cancer treatment}

Socioeconomic status (SES) has shown to substantially influence health and choice of therapy. Chapter 10 describes the influence of SES on breast cancer therapy. Using the Dutch cancer registry, we analyzed all 105,287 new onset stage I and II breast cancer patients diagnosed between 2011 and 2018. Surgical treatment occurred in 98,447 (of which 65,888 breast conserving treatment (BCT) and 32,559 mastectomy procedures) and 6,840 patients were not operated. We found no evidence that SES influences the indication for surgery. However, multivariate analysis showed that patients with a lower SES were less likely to undergo BCT instead of mastectomy.

In Chapter 11 we discussed the influence of SES on whether or not patients underwent immediate breast reconstruction (IBR) after mastectomy. We analyzed 32,559 new onset stage I and II breast cancer patients undergoing mastectomy, of which 26,463 did not receive IBR and 6,096 did receive IBR. SES was stratified into deciles and multivariate analysis showed that lower SES patients were less likely to undergo IBR after mastectomy. Corona virus disease 2019 (COVID-19) has put an enormous strain on healthcare systems worldwide. Chapter 12 describes the effects of COVID-19 on breast cancer surgical care in five hospitals in the center of the Netherlands. The study describes 217 consecutive cases during the first wave of the COVID-19 pandemic. The chapter showed that there was a decrease in the number of lower staged cancers operated while the number of higher staged cancers remained stable. Additionally, there were no changes in the risk of postoperative complications during the study period. 


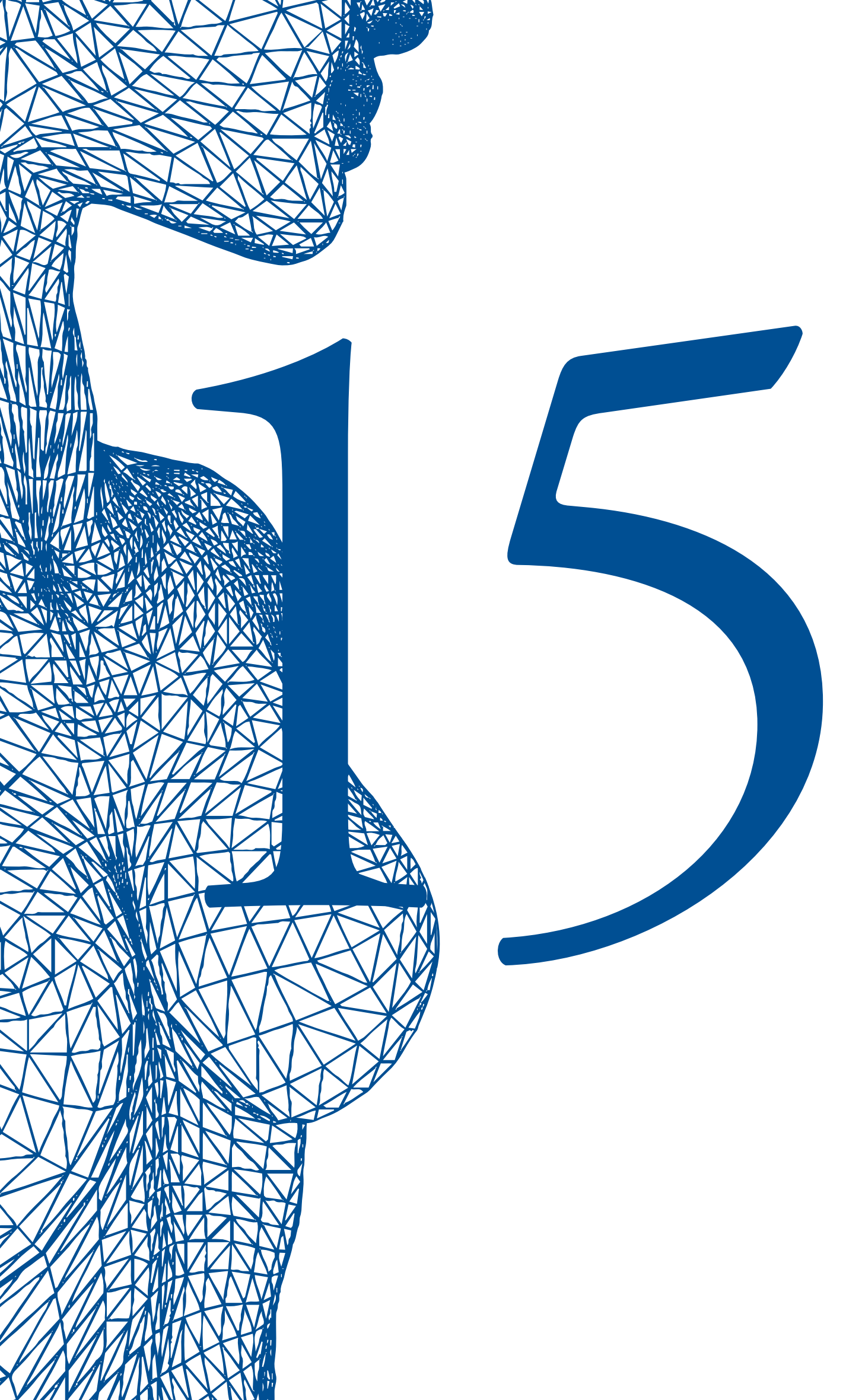


Chapter 15

Addenda 


\section{Samenvatting in het Nederlands}

\section{Deel I. Intraductale diagnose van aandoeningen van de borst}

Ductoscopie is een minimaal invasieve endoscopische techniek die live visualisatie van de melkgangen van de borst mogelijk maakt. Ductoscopie wordt vooral gebruikt voor de diagnose en behandeling bij patiënten met pathologische tepeluitvloed (PTU). Tegenwoordig wordt magnetic resonance imaging (MRI) ook gebruikt voor het uitsluiten van borstkanker bij patiënten met PTU. Hoofdstuk 2 vergelijkt de verschillende diagnostische methodes die momenteel beschikbaar zijn (mammografie, echografie, MRI, cytologie en ductoscopie) in een netwerk meta-analyse. Zesendertig studies met in totaal 3764 vrouwen met PTU ondergingen echografie, mammogram, MRI, cytologie en/of ductoscopie. De gepoolde sensitiviteit voor de detectie van maligniteit was respectievelijk $50 \%, 22 \%, 83 \%, 38 \%$ en $58 \%$. De gepoolde specificiteit was respectievelijk 69\%, 93\%, 76\%, 90\% en 92\% voor echografie, mammografie, MRI, cytologie en ductoscopie. Bovendien toonde subgroep meta-analyse, waarin ductoscopie werd vergeleken met MRI bij patiënten met PTU en een negatieve mammografie en echografie, aan dat de gevoeligheid van MRI hoger was (maar niet significant, $44 \%$ versus $76 \%$ ), maar ductoscopie vertoonde een significant hogere specificiteit ( $98 \%$ versus $84 \%$ ). Ductoscopie presteert daarom beter dan alle andere diagnostische methodes vanwege de superieure specificiteit in combinatie met de lage incidentie van maligniteit bij deze patiëntengroep, wat rond de $5 \%$ is.

De toevoeging van een biopsie-apparaat (soort schepnetje) aan ductoscopie maakt ingrepen (verwijdering van intraductale laesies) mogelijk. Deze interventie ductoscopie is een veelbelovende aanvulling op ductoscopie om de diagnostische mogelijkheden te verbeteren door een pathologische bevestiging van de oorzaak van PTU, wat meestal papillomen en verwijde melkgangen zijn. Hoofdstuk 3 beschrijft de bevindingen van een retrospectieve studie van 215 opeenvolgende patiënten die werden verwezen voor ductoscopie vanwege PTU zonder radiologische verdenking op maligniteit. Uiteindelijk waren er 151 succesvolle ductoscopiën uitgevoerd. Vijf van de zeven patiënten met maligniteit hadden verdachte afwijkingen bij ductoscopie die bij conventionele beeldvorming waren gemist. Bij 141 van de 144 patiënten zonder maligniteit hadden toonden geen verdachte afwijkingen tijden ductoscopie. Deze bevindingen, gecombineerd met het verdwijnen van PTU bij bijna de helft van de patiënten na ductoscopie, leidde tot een vermindering van het aantal geopereerde patiënten; slechts $28 \%$ van de patiënten met PTUwerd geopereerd. 
In Hoofdstuk 4 beschrijven we een methode om de gevoeligheid van ductoscopie voor het opsporen van maligniteit te verhogen. Autofluorescentie wordt veel gebruikt om de detectie van kankerprecursorlaesies van epitheliale oorsprong te verbeteren bij scopische procedures. Dit hoofdstuk beschrijft de haalbaarheid en het nut van autofluorescentie ductoscopie bij patiënten die zowel een preventieve als een therapeutische mastectomie ondergaan. Onze studie toonde aan dat autofluorescentie ductoscopie in staat was om (pre) carcinomateuze laesies te detecteren die niet werden gedetecteerd tijdens conventionele witlicht ductoscopie.

Hoofdstuk $\mathbf{5}$ is een studieprotocol dat de resterende uitdagingen van ductoscopie behandelt, namelijk lage gevoeligheid en verwijdering van kleine intraductale en epitheliale laesies. Dit hoofdstuk beschrijft een studieprotocol voor twee verschillende patiënten populaties: patiënten met PTU zonder radiologische verdenking van maligniteit en patiënten die (zowel profylactische als therapeutische) borstoperaties ondergaan. De eerste groep ondergaat gewone ductoscopie (wit licht), gevolgd door ductoscopie met narrow band imaging (NBI). Vervolgens worden verdachte intraductale laesies verwijderd met behulp van een intraductaal biopsie-apparaat. Als de extractie onvolledig is, zal de intraductale laserablatie de resterende laesie verwijderen. In de operatiegroep ondergaan patiënten direct voor operatie een reguliere ductoscopie, gevolgd door NBIductoscopie. Als er een (verdachte) laesie wordt gezien, wordt er een intraductale biopsie uitgevoerd en wordt het resterende deel van de laesie behandeld met laserablatie. De verschillende diagnostische instrumenten in beide patiëntengroepen worden geëvalueerd met de pathologische analyse als referentie. Bovendien zullen patiënten die intraductale laserablatie ondergaan, een follow-upperiode van 1 jaar hebben om te bepalen of PTU is gestopt. 


\section{Deel II: Minimaal invassieve behandeling bij aandoeningen van de borst}

Hoofdstuk 6 beschrijft de kosteneffectiviteit van ductoscopie voor de diagnose en behandeling van patiënten met PND zonder radiologische verdenking op maligniteit. De totale kosten voor ductoscopie, chirurgie en MRI bedroegen respectievelijk $€ 1.401,33$, $€ 6.494,27$ en $€ 822,13$ per ingreep. Uit het systematische onderzoek en de meta-analyse vonden we dat ductoscopie, ten opzichte van MRI, een lagere sensitiviteit had $(44 \%$ versus $76 \%$ ) maar een significant hogere specificiteit (84\% versus $98 \%$ ) en diagnostische accuratesse (95\% versus $83 \%$ ) voor het opsporen van maligniteit. Daarom hebben we een model geconstrueerd waarin patiënten met een positieve ductoscopie of MRI een operatie ondergingen. Omdat de kans groter was dat een MRI positief was (vanwege de lagere specificiteit), zouden meer patiënten een operatie ondergaan. Dit betekent dat een diagnostische route met MRI voor een patiënt met PTU gemiddeld $€ 2.070,27$ zou kosten, terwijl een diagnostische route met ductoscopie gemiddeld $€ 1.670,97$ zou kosten. Daardoor lijkt ductoscopie minder duur (gemiddeld $€ 399,30$ minder) en effectiever (hogere diagnostische nauwkeurigheid) ten opzichte van MRI. Daarnaast vergelijkt dit hoofdstuk de kosteneffectiviteit van chirurgie met ductoscopie voor de behandeling van PTU. Deze studie toonde aan dat PTU stopte bij respectievelijk 51,2\% en $100 \%$ van de patiënten die een ductoscopie of chirurgie ondergingen. Aangezien 48,3\% van de patiënten een operatie nodig heeft na ductoscopie, kost ductoscopie $€ 3.208,89$, terwijl de kosten van chirurgie $€ 6.494,27$ bedragen. Dit betekent dat ductoscopie minder effectief is, maar significant goedkoper dan chirurgie, en ductoscopie kan chirurgie bij bijna de helft van de patiënten voorkomen worden.

De door patiënt gerapporteerde uitkomsten van patiënten die ductoscopie ondergingen vanwege PTU zonder radiologische verdenking op maligniteit werden beschreven in hoofdstuk 7. In dit hoofdstuk analyseerde we de kwaliteit van leven bij 47 opeenvolgende patiënten met behulp van vragenlijsten (Breast-Q, EQ-5D-5L en SF-36) op de dag van ductoscopie, na twee weken, na drie en na zes maanden. De Breast-Q en EQ-5D-5L vertoonden geen significante veranderingen in kwaliteit van leven gedurende de followup na correctie voor aanwezigheid van PTU na ductoscopie en uiteindelijk diagnose. In de SF-36 vertoonde alleen het vitaliteitsdomein een lichte, maar significante afname door de tijd heen. Deze studie toonde aan dat de vragenlijsten geldig waren voor patiënten die ductoscopie ondergingen, maar er is meer onderzoek nodig om de symptoom specifieke problemen van patiënten met PTU in kaart te brengen.

Ductoscopiën worden in Nederland al tien jaar uitgevoerd bij patiënten met PTU zonder radiologische verdenking op maligniteit. Hoofdstuk 8 bevat de ontwikkelingen die er in het binnen- en buitenland zijn gemaakt en welke uitdagingen er nog liggen. 
Robot geassisteerde tepels parende mastectomie (RNSM) is een veelbelovende nieuwe techniek die het potentieel heeft om de morbiditeit van patiënten die borstamputatie nodig hebben te verminderen en tegelijkertijd het cosmetische uitkomsten te verbeteren. In Hoofdstuk 9 hebben we een systematische review en meta-analyse uitgevoerd die de complicaties beschrijft bij patiënten die een mastectomie ondergaan (zowel preventief als therapeutisch) met directe borstreconstructie. Negenenveertig studies met 13,886 patiënten die (R)NSM ondergingen werden in deze studie opgenomen en bij RNSM trad minder vaak (maar niet significant) een complicatie op vergeleken met traditionele mastectomie $(3,9 \%$ vs. $7,0 \%, \mathrm{p}=0,070)$. Daarom lijkt de minder invasieve RNSM een goed alternatief te zijn voor traditionele mastectomie. Er is echter meer onderzoek nodig om de oncologische veiligheid en de cosmetische uitkomst van deze nieuwe techniek te beoordelen. 


\section{Deel III. Invloed van socio-economische status en COVID-19 op de behandeling van borstkanker}

De sociaaleconomische status (SES) heeft een belangrijke invloed op gezondheid en therapiekeuze. Hoofdstuk 10 beschrijft de invloed van SES op de therapie van borstkanker. Met behulp van de Nederlandse kankerregistratie analyseerden we alle 105,287 nieuw ontstane stadium I en II borstkankerpatiënten gediagnosticeerd die tussen 2011 en 2018 waren gediagnostiseerd. Chirurgische behandeling vond plaats bij 98,447 (waarvan 65,888 mammasparende therapie (MST) en 32,559 een mastectomie ondergingen) en 6840 patiënten werden niet bediend. We vonden geen aanwijzingen dat SES invloed had op het wel of niet ondergaan van een operatie. Uit multivariate logistische regressie analyse bleek echter wel dat patiënten met een lagere SES minder snel MST ondergingen in plaats van mastectomie.

In Hoofdstuk 11 bespraken we de invloed van SES op het al dan niet ondergaan van directe borstreconstructie (IBR) na mastectomie. We analyseerden 32,559 nieuw ontstane stadium I en II borstkankerpatiënten die een mastectomie ondergingen, van wie 26,463 geen IBR kregen en 6.096 wel IBR. SES werd gestratificeerd in decielen en multivariate analyse toonde aan dat patiënten met een lagere SES minder snel IBR ondergingen na mastectomie.

Coronavirus disease 2019 (COVID-19) heeft wereldwijd een enorme druk gelegd op de gezondheidszorg. Hoofdstuk 12 beschrijft de gevolgen van COVID-19 op chirurgische zorg voor borstkanker in vijf ziekenhuizen in het midden Nederland. Deze studie beschrijft 217 opeenvolgende borstkankerpatiënten die tijdens de eerste golf van de COVID-19-pandemie zijn geopereerd. Dit hoofdstuk toonde aan dat er een afname was in het aantal patiënten met een laag stadium tumor terwijl het aantal patiënten geopereerde met een hoog stadium tumor stabiel bleef. Daarnaast toonde dit onderzoek aan dat er geen veranderingen in het risico op postoperatieve complicaties gedurende de onderzoeksperiode waren opgetreden. 


\section{Authors and affiliations}

\section{University Medical Center Utrecht}

Department of Surgery

E. de Bock

M.D. Filipe

W.M.U. van Grevenstein

M.C. Richir

M.R. Vriens

A.J. Witkamp

Department of Pathology

P.J. van Diest

S.I.S. Patuleia

Department of Medical Technology and Clinical Physics

F.E. Euwe

A.I. Rem

\section{St. Antonius Hospital, Nieuwegein}

Department of Surgery

D. Boerma

A. Doeksen

D. van Deukeren

S.L. Gerritsen

\section{Diakonessenhuis, Utrecht}

\section{Department of Surgery}
A. Pronk
E. Herman
G.M. Kuijper 
Rivierenland Hospital, Tiel

Department of Surgery

J.T. Heikens

Meander Medical Center, Amesfoort

Department of Surgery

T. A. Burghgraef

R. Geijtenbeek

P.M. Verheijen

Erasmus Medical Center, Rotterdam

Department of Plastic and Reconstructive Surgery

M.A.M. Mureau

Netherlands Comprehensive Cancer Organisation (IKNL)

Department of Research and Development

S. Siesling 


\title{
Review Committee
}

\author{
Prof. dr. I.H.M. Borel Rinkes
}

Department of Surgery, University Medical Center Utrecht

Prof. dr. R. Goldschmeding

Department of Pathology, University Medical Center Utrecht

Prof.dr. R.M. Pijnappel

Department of Radiology, University Medical Center Utrecht

Prof. dr. R.A.E.M. Tollenaar

Department of Surgery, Leiden University Medical Centre

Prof. dr. H.M. Verkooijen

Department of Radiation Oncology, University Medical Center Utrecht 


\section{List of Scientific publications}

Filipe MD, Patuleia SIS, Vriens MR, van Diest PJ, Witkamp AJ. Meta-analysis and costeffectiveness of ductoscopy, duct excision surgery and MRI for the diagnosis and treatment of patients with pathological nipple discharge. Breast Cancer Res Treat. doi: 10.1007/s10549-021-06094-x.

Filipe MD, de Bock E, et al. Impact of the COVID-19 pandemic on surgical colorectal cancer care in the Netherlands: a multicenter retrospective cohort study. J Gastrointest Surg. Doi: 10.1007/s11605-021-04936-z

Filipe MD, van Deukeren D, Kip M, et al. Effect of the COVID-19 pandemic on surgical breast cancer care in the Netherlands: a multicenter retrospective cohort study. Clin Breast Cancer. Published online August 7, 2020. doi:10.1016/j.clbc.2020.08.002

Filipe MD, Siesling S, Vriens MR, van Diest PJ, Witkamp AJ. The association of socioeconomic status on treatment strategy in patients with stage I and II breast cancer in The Netherlands. [accepted]

Filipe MD, Simons JM, L Moeliker, L Waaijer, MR Vriens, PJ van Diest, AJ Witkamp. Patient reported outcomes of patients undergoing ductoscopy for pathological nipple discharge without radiological suspicion of malignancy. Breast Cancer 10.1007/ s12282-020-01184-y.

Filipe MD, Makineli S, Vriens MR, van Diest PJ, Witkamp AJ. Ductoscopy for pathological nipple discharge (7 years later). [in preparation]

M Piek, E Postma, R van Leeuwaarde, JP de Boer, A Bos , C Lok , M Stokkel , $\underline{M}$ Filipe, I van der Ploeg. The effect of radioactive iodine therapy on ovarian function and fertility in female thyroid cancer patients: a systematic review and metaanalysis. Thyroid. Published online October 3, 2020:thy.2020.0356. doi:10.1089/ thy. 2020.0356

Filipe MD, E de Bock, EL Postma, OW Bastiaan, PPA Schellekens, MR Vriens, AJ Witkamp, MC Richir. Systematic review and meta-analysis comparing short-term effects of robot assisted nipple sparring mastectomy and conventional nipple sparring mastectomy. [Under review]

Filipe MD, Siesling S, Vriens MR, van Diest PJ, Witkamp AJ, Mureau MAM. Socioeconomic status significantly contributes to the likelihood of immediate postmastectomy breast reconstruction in the Netherlands: A nationwide study. Eur J Surg Oncol. Published online September 19, 2020. doi:10.1016/j.ejso.2020.09.016. 
Filipe MD, Makineli S, et al. Feasibility of narrow band imaging ductoscopy, intraductal biopsy and intraductal laser ablation in patients with pathological nipple discharge and patients with a high-risk for the development of breast cancer: a study protocol. [in preparation]

Waaijer L, Filipe MD, Simons J, et al. Detection of breast cancer precursor lesions by autofluorescence ductoscopy. Breast Cancer. Published online July 28, 2020. doi:10.1007/s12282-020-01136-6.

Filipe MD, Patuleia SIS, de Jong VMT, Vriens MR, van Diest PJ, Witkamp AJ. Network meta-analysis for the diagnostic approach to pathologic nipple discharge. Clin Breast Cancer. Published online June 8, 2020. doi:10.1016/j.clbc.2020.05.015.

Filipe MD, Waaijer L, van der Pol C, van Diest PJ, Witkamp AJ. Interventional ductoscopy as an alternative for major duct excision or microdochectomy in women suffering pathologic nipple discharge: a single-center experience. Clin Breast Cancer. 2020;20(3):e334-e343. doi:10.1016/j.clbc.2019.12.008.

Filipe MD, Meijers WC, Rogier van der Velde A, de Boer RA. Galectin-3 and heart failure: prognosis, prediction \& clinical utility. Clin Chim Acta. 2015;443:48-56. doi:10.1016/j.cca.2014.10.009.

Filipe $\mathrm{MD}^{*}$, de Bock E*, Herman E, et al. Lesson learnt from surgical patients requiring ICU during the first wave of the COVID-19 pandemic. Submitted

Filipe MD, Donners S, et al. Impact of the COVID-19 pandemic on vascular surgery care in the Netherlands: a multicenter retrospective cohort study. Submitted

Filipe MD, van Tuyll van Serooskerken, et al. Influence of the COVID-19 pandemic on pediatric surgical practice. Submitted

De Bock E, Filipe MD, et al. Quality of life in IBD patients during the COVID-19 pandemic in the Netherlands. Pending revisions

De Bock E, Filipe MD, et al. Factors affecting 30 day postoperative complication after emergency surgery during the COVID-19 outbreak: a multicentre study. Submitted. 


\section{Dankwoord}

Direct nadat ik mijn studie geneeskunde beëindigde, startte ik met mijn onderzoek naar ductoscopiën. Zelf had ik weinig tot geen ervaring met onderzoek en ik had geen idee waar het onderzoek naar toe zou gaan. Het enige wat ik op dat moment wist is dat, in samenwerking met de TU Delft, een biopsie device ontwikkeld ging worden voor ductoscopie. Gezien mijn beperkte kennis en ervaring met onderzoek, besloot ik, kort nadat ik begonnen was aan mijn onderzoek, ook een master epidemiologie te doen aan de Universiteit van Amsterdam. Afgelopen jaren heb ik zelfstandig leren werken en kritisch na leren denken. Ook heb ik een ontzettende leuke en leerzame tijd gehad met de medepromovendi. Graag wil ik een goed aantal mensen die direct en indirect betrokken zijn geweest bij de totstandkoming van dit proefschrift.

Mijn hooggeleerde promotor, prof. dr. Vriens, beste Menno. Ik ben meer dan geëerd dat jij mijn promotor wilde zijn. Jij hebt mij altijd enorm geënthousiasmeerd om out of the box te denken en aangemoedigd om uitdagende projecten aan te gaan ("we gaan er weer wat moois van maken!" en "gaan we doen!"). Van jou heb ik geleerd dat de chirurgie topsport is; het is presteren op het hoogste niveau en niet alleen bezig zijn met één ding maar zo veel mogelijk kansen aangaan die je toegereikt krijgt. Ik kijk zeer uit naar toekomstige samenwerkingen.

Mijn hooggeleerde promotor, prof. dr. van Diest, beste Paul. Jij hebt mij heel goed door mijn promotietraject begeleid. Ook heb jij mij geënthousiasmeerd om breed te denken en uitdagingen aan te gaan. Dit eerste zorgde er wel voor dat jouw begeleiding iets meer toegespitst moest worden op focussen van mijn energie en tijd.

Mijn hooggeachte copromotor, dr. Witkamp, beste Arjen. Van jou heb ik ontzettend veel geleerd. Naast chirurg, ben jij ook bestuurder en wetenschapper. Als wetenschapper heb jij mij geleerd om focus te houden binnen mijn nogal brede interesses. Jij hebt mij geleerd om pragmatisch en praktisch klinisch onderzoek te verrichten. Ook ben ik ontzettend dankbaar dat jij mij begeleidt als wetenschapper maar ook betrekt bij op jouw bestuurlijke nevenfunctie binnen de NVCO. Jouw bestuurlijke vaardigheden en jouw persoonlijke eigenschappen om mensen mee te krijgen heb ik tijdens de pandemie, en daarmee laatste maanden van mijn promotie, steeds meer en beter leren kennen.

Geachte leden van de beoordelingscommissie. Hartelijk dank voor uw kritische blik op dit proefschrift.

Geachte leden van de oppositiecommissie, hartelijk dank voor om de tijd te nemen en om bereid te zijn om te opponeren. 
Prof. Breedveld en dr. Sakes. Vanuit de TU Delft zijn jullie essentieel geweest in het ontwikkelen van de biopsienaald voor ductoscopie. Ik wil jullie daarvoor enorm bedanken.

Dr. Richir, beste Milan. Met jou raakte ik iets later in mijn promotie betrokken maar we hebben alsnog een hele nauwe samenwerking gehad op het gebied van COVID-19 en robot borst chirurgie. Bij jou zag ik enorme energie en gedrevenheid om onderzoek doen en dat je niet vies bent om eventjes door te werken. Ook heb jij mij een nieuwe definitie van het woord "flow" gegeven, waarvoor ik jou erg dankbaar ben en nooit zou vergeten. Samen hebben we iets best bijzonders bereikt in hele korte tijd. Ik kijk erg uit naar verdere samenwerkingen.

COVID-chirurgie groep midden Nederland. Jullie wil ik bedanken om in de moeilijke COVID-pandemie tijd onderzoek naar COVID op chirurgische patiënten mogelijk te maken. Heel veel dank dr. Pronk, dr. Boerma, dr. Heikens en dr. Verheijen. Ook wil ik Thijs Burghgraef en Ellen de Bock bedanken om het onderzoek op te zetten en gaande te houden. Zonder jullie was dit niet mogelijk geweest. Als laatste, maar ook ontzettend belangrijk, wil ik de (arts)onderzoekers bedanken die geholpen hebben met de data: Daan, Simone, Tim, Saskia, Ritch, Sofie, Marijn, Kim, Sarah, Desiree, Eline H, Joshua en Eline V.

OnCovid! stuurgroep. Van jullie heb ik ontzettend veel geleerd over de complexiteit van de zorg in Nederland. Ook heb ik ontzettend van dichtbij geleerd de uitdaging die op bestuurlijk niveau heerst tijdens een grote crisis. Verder heb ik bewondering voor de verschillende leden die naast hun drukke banen tijd en energie stoppen en de verantwoordelijkheid voelen voor het waarborgen van de oncologische zorg in heel Nederland tijdens de COVID-pandemie. Het is een grote eer om aan dit team deel te mogen nemen. Dank aan prof. dr. Tollenaar, prof. dr. Verheij, dr. Duijvendijk, dr. Hartgerink en (uiteraard) dr. Witkamp.

DMT, lotgenoten tijdens COVID. Jullie heb ik slechts enkele keren in het live gezien echter na zo veel virtuele sessies heb ik het idee gekregen dat we elkaar best goed kennen. Vooral na de aprés-zoomsessies. Ook al waren ze niet altijd gepaard met lekkere broodjes. Dank Angelique en Robert!

Prof. dr. Siesling, beste Sabine, hartelijk dank voor je inzet en betrokkenheid bij de studies over socio-economische status en ondergane borstkanker behandeling. Ik kijk uit naar verdere samenwerkingen in de toekomst.

Prof. dr. Mureau, beste Marc. Hartelijk dank voor je inzet en betrokkenheid bij de studie over socio-economische status en het aangaan van reconstructie na borstamputatie. Ook wil ik jou bedanken om mij te betrekken bij verdere plastisch chirurgisch onderzoek.

Dr. Postma, beste Emily. Ook jou wil ik bedanken om mij de kans te geven om een kijkje te kunnen nemen in de endocriene kant van de chirurgie. 
Prof dr. de Borst en Simone Donners. Jullie wil ik bedanken om mij de kans te geven om een kijkje te kunnen nemen in de vaatchirurgie.

Prof dr. Leenen, dr. van Baal en Tim Kobes. Jullie wil ik bedanken om mij de kans te geven om een kijkje te kunnen nemen in de traumachirurgie.

Prof. dr. Oldenburg. Jou wil ik bedanken voor het waar maken van de chirurgische IBD-studie.

Dr. Tygat en Sofie van Tuyll van Serooskerken. Jullie wil ik enorm bedanken om mij de kans te geven om een kijkje te kunnen nemen in de kinderchirurgie

Dr. Bastiaan, beste Okan. Jou wil ik bedanken om mij te betrekken met het onderwijs gedeelte van de chirurgie.

Ellen, jij was en bent mijn tweede paar ogen en rechterhand voor het COVID-onderzoek. We hebben samen heel snel kunnen schakelen en daarmee heel veel kunnen bereiken. Ik heb er alle vertrouwen in dat jij de eerste persoon in Nederland zult zijn die op COVID (en chirurgie) promoveert!

Seher, jij neemt het stokje over. Ik heb er alle vertrouwen in dat je er groot succes van gaat maken. Zet'm op!

Beste dr van der Ploeg, beste Iris. Jou wil ik ook bedanken om mij te verwelkomen in het AvL. Ook mijn nieuwe collegae in het AvL wil ik bedanken voor de warm ontvangst.

Froukje Euwe. Jou wil ik bedanken voor de nauwe samenwerking om de klinische fase van het ductoscopie onderzoek waar te maken. Zonder jouw inzet was dit absoluut niet mogelijk geweest.

Yvonne en Suzanne. Jullie zorgde altijd met veel enthousiasme niet alleen dat de patiënten zorg goed ging voor ductoscopie maar ook de hele logistiek over het onderhoud van de ductoscopen. Verder zijn jullie altijd geïnteresseerd geweest in de experimenten waar ik mee bezig was. Dank voor jullie inzet.

Fatiha, Romy en Mariëlle. Jullie wil ik hartelijk bedanken voor jullie hulp en ondersteuning.

Susanna, jou wil ik bedanken voor onze samenwerking op onze (netwerk) meta-analyse en ons PND RNA onderzoek. Samen met Mariëlle zorgde jullie ervoor dat het onderzoek goed verliep. Hartelijk dank voor jullie inzet. Ook wil ik dr. Moelans bedanken voor de analyses van de samples. 
Mijn kamer genoten, de eerste helft gaat aan Feike Kingma, Arjen van der Veen en Emma Gertsen om mij een plekje te geven in de Upper-GI-kamer in de kelder van het Stratenum. De sfeervolle kamer zat vol met foto's die als inspiratie hebben gediend voor mijn onderzoek (Feike: behalve de ingezoomde groepsfoto van de wetenschapsdag). De tweede helft gaat naar Dirk-Jan van Beek, Marieke Walma, Tim Kobes en Arthur Swiet in de derde verdieping van de PromoToren. Hier heb ik veel kunnen filosoferen over het leven na het uitbreiden van onze collectie.

Ook wil ik de docenten van de Master Evidence Based Practice aan de Universiteit van Amsterdam. Dat jullie het avondeten geregeld moesten overslaan zorgde ervoor dat ik de analyses in dit proefschrift kon doen.

Musjeddi, sinds 2010 kennen we elkaar als de leukste groep uit het noorden. Jullie zijn een leuke afwisseling van de medische bubbel waarin ik me bevind, is iets wat ik steeds meer leer te waarderen. Waarvoor dank Getje, Kim K, Busu, Trunnu, Ribba, Jos B, Pries, PK, MmG, Kinna en Djènn (uiteraard ook Tjerk en André).

Hoekers, place to be in het noorden. Jullie hebben mijn een thuis gegeven in het hoge Noorden. Ontzettend bedankt voor een fantastische tijd around the corpsner met veel humor! Groen en blauwe groet aan Happy, Wintie, Cage, Mooie Dingen, Wally, De Don, Willie, Jappa, VP en de Baron.

Wir sind, de sportieve groep. Jullie hebben mij echt leren sporten. Hartelijk dank Birt, Minkie, Sanctor, L Bouncing K, Thijs, Evo en Fleuro.

DB'13. Met jullie heb ik een fantastisch en onvergetelijk mooi jaar gehad in het hok en daarbuiten. Enorm bedankt Lair, Eef, Q, Vinnie, Rensol, Britty, Eef (bis) en Mel C!

Shorehouse, de internationals. Met jullie heb ik altijd een ontzettend leuke tijd gehad voor en na de studie. Veel dank voor de mooie tijden Chubby B, EJ, CD, JJ, Kiki (Big A) en WP.

Lieve Mama, dank voor de onvoorwaardelijke steun en vertrouwen. Jij had goed in de gaten dat ik niet helemaal op mijn plek zat toen ik informatica studeerde en hemel en aarde hebt bewogen om mij op mijn plek te krijgen bij de studie geneeskunde in Groningen. Ook na mijn studie heb je mij volledig gesteund.

Wills, mijn broertje tevens sportgenoot, studiegenoot, coauteur, mede chirurgische promovendus en paranymf. Zoals je merkt hebben veel dingen gemeenschappelijk. Met jou kan ik alles delen op werkniveau (gezamenlijke artikelen) en op sociaal vlak (NP, F7, $\mathrm{VaP}$ en $\mathrm{YW}$ ). We hebben altijd met alles kunnen levellen en ik heb er alle vertrouwen in dat we dit erin houden. 
Liefste Marianne. Vaak heb ik gezegd dat je een beetje medium OK bent. Zelf weet je wellicht dat het heel mogelijk iets meer is dan dat. Ik hou ontzettend veel van je en wij zijn slechts bij het begin van een fantastisch leven. Ontzettend bedank voor de steun die je mij altijd hebt geboden. Wij blijven er voor elkaar. 


\section{About the author}

Mando Dyko Filipe was born on the 24th of December 1989 in Utrecht, The Netherlands, as oldest son. Together with one younger brother, Mando grew up in the Costa Blanca in Spain. After graduating from I.E.S. Antoni Llidó, he started computer science in Universidad Politécnica de Valencia, Spain. After 2 years, Mando started his medical study in University of Groningen, the Netherlands. While in Groningen, Mando enjoyed playing football (GSAVV Forward), tennis (GSTC) and wakeboarding (GSWV the Bares). He also joined a fraternity $(\mathrm{VaP})$. During the medical study, his surgical interests were further confirmed with extracurricular internships in cardiothoracic surgery and oncological surgery in Groningen.

During the last clinical internship (semi-arts) in the University Medical Center of Utrecht (UMCU) in de department of surgical oncology, Mando got involved in ductoscopy research thanks dr. A.J. Witkamp. After obtaining his medical degree, Mando started a fulltime $\mathrm{PhD}$ on ductoscopy under supervision of prof. dr. M.R. Vriens, prof. dr. P.J. van Diest and dr A.J. Witkamp. The PhD program is a joint venture of the UMCU and the Technical University of Delft, which was an ideal fit because of Mando's passion for clinical research and affinity with technology.

During his $\mathrm{PhD}$, Mando also obtained a postgraduate master in clinical epidemiology at University of Amsterdam, the Netherlands. This appeared to very useful for the settingup, analysing and publishing COVID-19 related research under supervision of dr. M.C. Richir. Additionally, Mando was involved in the developing a measures database to ensure the continuity of oncological care during the second wave of the COVID-19 pandemic in the Netherlands.

Mando aspires to become a surgeon while continuing to develop himself in clinical research. Additionally, Mando aims to combine his surgical career with triathlons and spending time with friends and family. 
